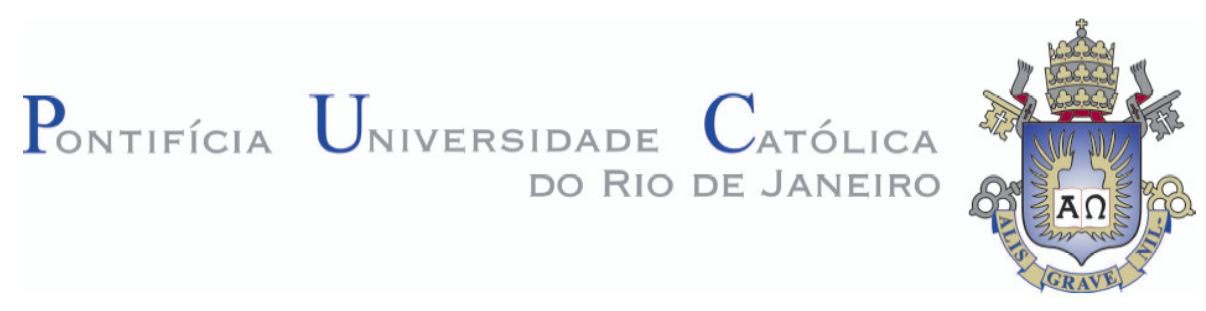

Ismael Andrade Pimentel

\title{
Um Método Meshfree Adaptativo de Advecção para Problemas de Fluxo Bifásico de Fluidos Incompressíveis e Imiscíveis em Meios Porosos Heterogêneos Tridimensionais
}

Tese de Doutorado

Tese de Doutorado apresentada ao Programa de Pósgraduação em Engenharia Mecânica da Pontifícia Universidade Católica do Rio de Janeiro como requisito parcial para a obtenção do título de Doutor em Engenharia Mecânica.

Orientador: Prof. Marcelo de Andrade Dreux

Co-orientador: Prof. Bruno Feijó

Rio de Janeiro

Abril de 2015 


\title{
Pontifícia Universidade Católica \\ DO RIO DE JANEIRO
}

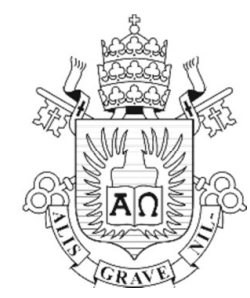

Ismael Andrade Pimentel

\begin{abstract}
Um Método Meshfree Adaptativo de Advecção para Problemas de Fluxo Bifásico de Fluidos Incompressíveis e Imiscíveis em Meios Porosos Heterogêneos Tridimensionais

Tese de Doutorado apresentada ao Programa de Pósgraduação em Engenharia Mecânica da Pontifícia Universidade Católica do Rio de Janeiro como requisito parcial para a obtenção do título de Doutor em Engenharia Mecânica.
\end{abstract}

Prof. Marcelo de Andrade Dreux Orientador Departamento de Engenharia Mecânica - PUC-Rio

Prof. Bruno Feijó Co-orientador Departamento de Engenharia Informática - PUC-Rio Dr. Mauricio Kreczmarsky Guimarães Meinicke Tecgraf - PUC-Rio

Dr. Nelson Inoue Grupo de Tecnológico e Engenharia de Petróleo - PUC-Rio

Prof. Luiz Eduardo Azambuja Sauerbronn UFRJ

Dr. Pedro Mário Cruz e Silva

Tecgraf -PUC-Rio

Prof. Esteban Walter Gonzalez Clua

UFF

Prof. José Eugênio Leal Coordenador Setorial do Centro Técnico Científico - PUC-Rio

Rio de Janeiro, 28 de Abril de 2015 
Todos os direitos reservados. É proibida a reprodução total ou parcial do trabalho sem autorização da universidade, do autor e do orientador.

\section{Ismael Andrade Pimentel}

Graduou-se em Engenharia de Computação pela Universidade Santa Úrsula (Rio de Janeiro, RJ) em 2006. . Concluiu o Mestrado na IPRJ-UERJ (Rio de Janeiro, Brasil) em 2008 na área de Modelagem Computacional.

Ficha Catalográfica

Pimentel, Ismael Andrade

Um método meshfree adaptativo de advecção para problemas de fluxo bifásico de fluidos incompressíveis e imiscíveis em meios porosos heterogêneos tridimensionais / Ismael Andrade Pimentel ; orientador: Marcelo de Andrade Dreux ; co-orientador: Bruno Feijó. - 2015.

170 f. : il. (color.) ; $30 \mathrm{~cm}$

Tese (doutorado)-Pontifícia Universidade Católica do Rio de Janeiro, Departamento de Engenharia Mecânica, 2015.

Inclui bibliografia

1. Engenharia mecânica - Teses. 2. Simulação de fluidos. 3. Métodos numéricos. 4. Mecânica computacional. 5. Computação gráfica. 6. Meios porosos heterogêneos. I. Dreux, Marcelo de Andrade. II. Feijó, Bruno. III. Pontifícia Universidade Católica do Rio de Janeiro. Departamento de Engenharia Mecânica. IV. Título.

CDD: 621 


\section{Agradecimentos}

À Deus, por tudo. Aos meus caros orientadores, Professor Marcelo Dreux e o Professor Bruno, pelo carinho e por todo apoio familiar. Os dois foram muito mais que apenas meus orientadores, nesta trajetória, foram um modelo no qual pude me espelhar. Motivaram-me, em todos os momentos, como modelo de integridade, profissionalismo e generosidade. Agradeço especialmente pelo apoio, orientação, amizade e paciência. Agradeço ainda por vossa transparência e por suas mentes brilhantes. O olhar inovador dos senhores e todas as contribuições desafiadoras foram fundamentais para a conclusão deste trabalho.

Ao Professor Nelson Inoue pela motivação, pelo companheirismo e pela partilha generosa de seu conhecimento no IMEX. Agradeço imensamente a convivência que tanto contribui para o meu crescimento profissional, acadêmico e sobretudo pessoal. Ao Doutor Mauricio Meinicke, pelos anos de companheirismo no grupo de pesquisa.

Aos amigos Elvis Chantre e Edson Encarnação, por me fazerem estar sempre em conexão com minha terra natal, fazendo parecer que Cabo Verde é perto daqui. Aos amigos Bruno Kassar, Ruben Gomes, Carlos Geraldo, Herberth, Danmer, José e Ana Carolina Almeida por todo apoio e amizade. Durante este tempo, sempre estiveram dispostos a ajudar e a compartilhar ideias.

Ao GTEP pelo acolhimento e por ter concedido o uso do software IMEX, que viabilizou uma importante contribuição para esta pesquisa. Em especial ao professor Doutor Sergio Fontoura. Agradeço também a todos os amigos do GTEP, Rafael, Guilherme, Daniel, Juan e Carlos Emmanuel, pelo dia-a-dia, pela troca de experiência e por me proporcionarem uma convivência leve e um ambiente tão produtivo.

Ao CNPq e a PUC-Rio pelo auxilio financeiro. 


\section{Resumo}

Pimentel, Ismael Andrade; Dreux, Marcelo; Feijó, Bruno. Um método meshfree adaptativo de advecção para problemas de fluxo bifásico de fluidos incompressíveis e imiscíveis em meios porosos heterogêneos tridimensionais. Rio de Janeiro, 2015. 170p. Tese de Doutorado Departamento de Engenharia Mecânica, Pontifícia Universidade Católica do Rio de Janeiro.

Esta tese propõe um método meshfree adaptativo de advecção para problemas de fluxo bifásico de fluidos incompressíveis e imiscíveis em meios porosos heterogêneos tridimensionais. Este método se baseia principalmente na combinação do método Semi-Lagrangeano adaptativo com interpolação local meshfree usando splines poliharmônicas como funções de base radial. O método proposto é uma melhoria e uma extensão do método adaptativo meshfree AMMoC proposto por Iske e Käser (2005) para modelagem $2 D$ de reservatórios de petróleo. Inicialmente este trabalho propõe um modelo em duas dimensões, contribuindo com uma melhoria significativa no cálculo do Laplaciano, utilizando os métodos meshfree de Hermite e Kansa. Depois, o método é ampliado para três dimensões $(3 D)$ e para um meio poroso heterogêneo. O método proposto é testado com o problema de five spot e os resultados são comparados com os obtidos por sistemas bem conhecidos na indústria de petróleo.

\section{Palavras-chave}

Simulação de Fluidos; Métodos Numéricos; Level Set; Semi-Lagrangeano; Advecção; Meshfree; Adaptativo; Mecânica Computacional; Computação Gráfica; Meios Porosos Heterogêneos. 


\section{Abstract}

Pimentel, Ismael Andrade; Dreux, Marcelo (Advisor); Feijó, Bruno (Co Advisor). An adaptive meshfree advection method for two-phase flow problems of incompressible and immiscible fluids through threedimensional heterogeneous porous media. Rio de Janeiro, 2015. 170p. D.Sc. Thesis - Departamento de Engenharia Mecânica, Pontifícia Universidade Católica do Rio de Janeiro.

This thesis proposes an adaptive meshfree advection method for two-phase flow problems of incompressible and immiscible fluids through three-dimensional heterogeneous porous media. This method is based mainly on a combination of adaptive semi-Lagrangian method with local meshfree interpolation using polyharmonic splines as radial basis functions. The proposed method is an improvement and extension of the adaptive meshfree advection scheme AMMoC proposed by Iske and Käser (2005) for 2D oil reservoir modeling. Initially this work proposes a model in two dimensions, contributing to a significant improvement in the calculation of the Laplacian, using the meshfree methods of Hermite and Kansa. Then, the method is extended to three dimensions (3D) and a heterogeneous porous medium. The proposed method is tested with the five spot problem and the results are compared with those obtained by well- known systems in the oil industry.

\section{Keywords}

Fluid Simulation; Numerical Methods; Level Set; Semi-Lagrangian; Advection; Meshfree; Adaptive; Computational Mechanics; Computer Graphics; Heterogeneous Porous Media. 


\section{Sumário}

1.1. Considerações Gerais .......................................................... 16

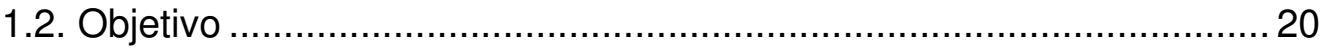

1.3. Trabalhos Relacionados ........................................................... 20

1.3.1 Trabalhos relacionados referentes ao método Level Set .................20

1.3.2. Trabalhos relacionados referentes ao método Semi-

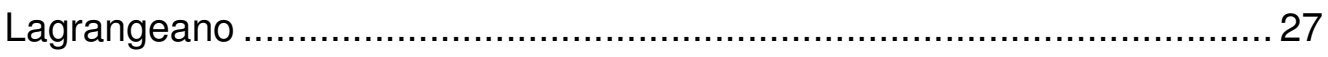

1.3.3. Trabalhos relacionados referentes aos métodos meshfree..............30

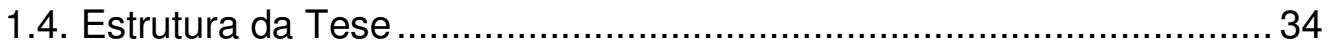

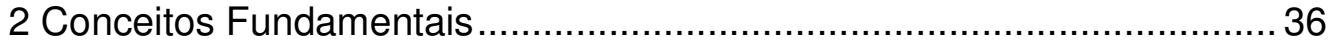

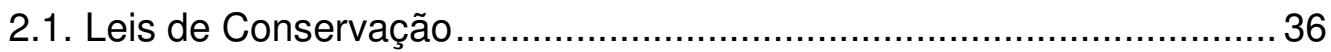

2.1.1. Lei de Conservação de Massa .................................................. 36

2.1.2. Equação da Quantidade de Movimento ........................................ 38

2.1.3. Equação de Energia ......................................................... 38

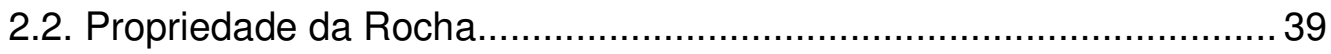

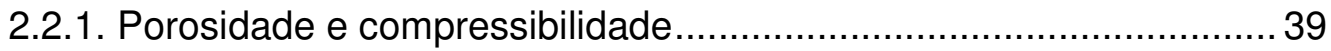

2.2.2. Permeabilidade Absoluta........................................................ 40

2.3. Lei de Darcy .................................................................... 40

2.4. Leis de Modelo de Brooks e Corey e Van Gebutchen........................ 40

2.5. Derivada Material ................................................................. 43

2.6. Advecção....................................................................... 44

2.7. Abordagens Lagrangeana e Euleriana ....................................... 44

2.8. Conceito e Formulação do Método Level Set.................................... 46

3 Formulação do método Semi-Lagrangeano de advecção ...................... 50

3.1. Algoritmo e o Fluxograma do Modelo com Malha ............................5 54

3.2. Algoritmo e o Fluxograma do Modelo Meshfree ..............................58

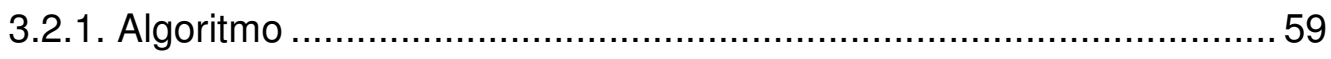

3.3. Interpolação Spline Poliharmônica ............................................. 64

3.4. Vantagens e desvantagens do Método Semi-Lagrangeano................66 66

4 Formulação dos métodos meshfree ............................................. 67

4.1. Métodos meshfree ............................................................... 68

4.1.1. Aproximação por método antissimétrico de Kansa .......................69 69 
4.1.2. Aproximação pelo método simétrico de Hermite

5 Novo modelo de fluxo multifásico de fluidos imiscíveis e incompressíveis em meios porosos heterogêneos.

5.1. Nova formulação do modelo de fluxo bifásico de fluidos incompressíveis e imiscíveis em meios porosos heterogêneos tridimensionais.

5.2. Estratégias para representação do modelo proposto ......................... 89

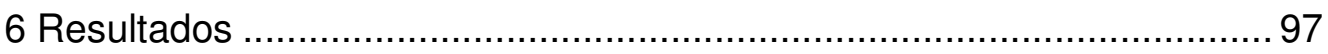

6.1. Método Semi-Lagrangeano 1D e 2D ............................................ 97

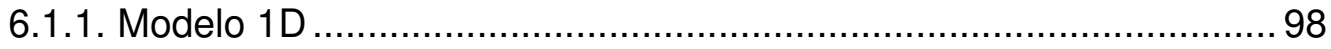

6.1.2. Modelo 2D .......................................................................... 100

6.2. Resultados do modelo adaptativo meshfree do problema 2D .......... 109

6.2.1. Resultados do modelo meshfree ........................................... 109

6.2.2. Resultados do Modelo Adaptativo ........................................... 124

6.3. Resultados do novo modelo bifásico imiscível e incompressível num meio poroso heterogêneo em domínio tridimensional.................... 131

6.3.1. Resultados do Novo Modelo Proposto Utilizando uma Geometria Regular.................................................................................... 136

6.3.2. Resultados do novo modelo proposto utilizando uma geometria

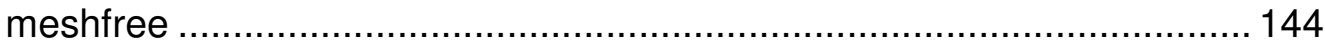

7 Conclusão e Trabalhos Futuros ..................................................... 150

8 Referências Bibliográficas ...................................................... 153

9 Apêndices............................................................................. 161

9.1. Apêndice A ................................................................. 161

Modelo de five spot real usando o IMEX ....................................... 161

Simulação do Problema real de five-spot utilizando o IMEX ................... 162 


\section{Lista de Figuras}

Figura 1: Superfície Opaca Gerado pelo Método Level Set (Guedelman et al, 2005)

Figura 2: Rayleigh - Taylor, 4 e 5 fases (Losasso et al, 2006) 22

Figura 3: Rayleigh - Formação de Onda (Geiger et al, 2006) 23

Figura 4: Interface e Velocidade de Campo para Diferentes Instantes De Tempo (Vorgelegt Von, 2013)

Figura 5: (Esquerda) a geração de torneira de bolhas através de retenção de ar; (Centro) Uma fonte para gerar pequenas bolhas que se fundem, interagem e crescem com o movimento do objeto; (Direita) Uma hélice gerando um helicoidal. (Patkar et al, 2013)

Figura 6: Propagação do fluido gás através da vaporização, dada pelo campo de pressão e pelo campo de temperatura (Gjennestad e Munkejord, 2015)

Figura 7: Derramamento de água em uma caixa a partir de duas fontes de fluxo opostas (Lentine et al, 2012)

Figura 8: Dois navios em tempestade próxima de uma ilha (English et al, 2013)

Figura 9: Uma grande cena de oceano simulado com o método twoway coupling entre os métodos de partículas SPH e o Level Set. Uma simulação de ar secundária também é usada para gerar uma segunda camada de detalhes com espuma e neblina provenientes de partículas do método SPH (Losasso et al, 2008)

Figura 10: Interação fluido-objeto. Um barco passando por baixo da ponte (Esquerda). Um segundo barco colidindo com a ponte (meio e direita. (Akinci et al, 2012)

Figura 11: Interação de partículas da água com a baleia causando efeitos secundários como espuma, spray e borbulhas. (Chentanez et al., 2014)

Figura 12: Solidificação da lava (Somakhin et al., 2014)

Figura 13: Relação entre a saturação e a permeabilidade relativa usando os modelos de Brooks-Corey e Van Genuchten, Solidificação. (Marshall, 2009)

Figura 14: Abordagem Euleriano 45

Figura 15: Abordagem Lagrangeano 46

Figura 16: Level Set $\quad 47$

Figura 17: Abordagem Semi-Lagrangeano 50

Figura 18: Upstream Point $\quad 51$ 
Figura 19: - Stencil Points.

Figura 20: Fluxograma

Figura 21: Fluxograma de um simples modelo numérico de reservatórios (Adalberto et al, 2006).

Figura 22: Propagação de uma Onda Senoidal Unidirecional (Costa et at, 2004)

Figura 23: Propagação de uma Onda Senoidal Unidirecional utilizando o método Semi-Lagrangeano

Figura 24: Campo de Pressão (Iske e Käser, 2005)

Figura 25: Campo de Velocidade(Iske e Käser, 2005)

Figura 26: Saturação no problema five spot em 6 instantes de tempo (t) (Iske e Käser, 2005)

Figura 27: Campo de Pressão. Resultado obtido usando o modelo da tese

Figura 28: Campo de Velocidade. Resultado obtido usando o modelo da tese

Figura 29: Campo de Pressão-Velocidade sobreposto. Resultado obtido usando o modelo da tese

Figura 30: Problema de five spot. Resultado obtido usando o modelo da tese: Figura a: ${ }^{t=t_{0}}$, Figura b: ${ }^{t=t_{420}}$, Figura c: ${ }^{t=t_{840}}$, Figura d: $t=t_{1260}$, Figura e: ${ }^{t=t_{1680}}$, Figura f: ${ }^{t=t_{2100}}$

Figura 31: Campo de pressão do problema five spot. Resultado obtido usando o modelo Semi-Lagrangeano meshfree

Figura 32: Campo de velocidade do problema five spot. Resultado obtido usando o modelo Semi-Lagrangeano meshfree

Figura 33: Forma de colocação de pontos na fronteira

Figura 34: Saturação do modelo adaptativo do Problema five spot

Figura 35: Saturação do modelo adaptativo do problema five spot utilizando o método de Hermite

Figura 36: Saturação do modelo adaptativo do problema five spot utilizando o método de Hermite

Figura 37: Saturação do modelo adaptativo do problema five spot, utilizando o método de Hermite

Figura 38: Saturação do modelo adaptativo do problema five spot, utilizando o método de Hermite

Figura 39: Saturação do modelo adaptativo do problema five spot, utilizando o método de Hermite

Figura 40: Saturação do modelo adaptativo do problema five spot, utilizando o método de Kansa

Figura 41: Saturação do modelo adaptativo do problema five spot, 
utilizando o método de Kansa

Figura 42: Saturação do modelo adaptativo do problema five spot, utilizando o método de Kansa

Figura 43: Saturação do modelo adaptativo do problema five spot, utilizando o método de Kansa

Figura 44: Saturação do modelo adaptativo do problema five spot, utilizando uma malha regular

Figura 45: Saturação do modelo adaptativo do problema five spot, utilizando uma malha regular

Figura 46: Saturação do modelo adaptativo do problema five spot, utilizando uma malha regular

Figura 47: Saturação do modelo adaptativo do problema five spot, utilizando uma malha regular

Figura 48: Saturação do modelo adaptativo do problema five spot, utilizando pontos Voronoi

Figura 49: Saturação do modelo adaptativo do problema five spot, utilizando pontos Voronoi

Figura 50: Saturação do modelo adaptativo do problema five spot, utilizando pontos Voronoi

Figura 51: Saturação do modelo adaptativo do problema five spot, utilizando pontos Voronoi

Figura 52: Porosidade do meio heterogêneo SPE 10, modelo 2.

Figura 53: Visão do logaritmo da permeabilidade absoluta do meio poroso heterogêneo SPE 10, modelo 2 do SPE66599 na direção X.

Figura 54: Visão do logaritmo da permeabilidade absoluta do meio poroso heterogêneo SPE 10, modelo 2 do SPE66599 na direção Y.

Figura 55: Visão do logaritmo da permeabilidade absoluta do meio poroso heterogêneo SPE 10, modelo 2 do SPE66599 na direção Z.

Figura 56: Saturação no meio poroso homogêneo em $\mathrm{T}=1$.

Figura 57: Saturação no meio poroso homogêneo em T = 200.

Figura 58: Saturação no meio poroso homogêneo em $\mathrm{T}=300$.

Figura 59: Saturação no meio poroso homogêneo em $\mathrm{T}=400$.

Figura 60: Saturação no meio poroso heterogêneo em $\mathrm{T}=1$.

Figura 61: Saturação no meio poroso heterogêneo em T = 200.

Figura 62: Saturação no meio poroso heterogêneo em T = 300.

Figura 63: Saturação no meio poroso heterogêneo em $\mathrm{T}=400$.

Figura 64: Saturação no meio poroso heterogêneo em T = 2000.

Figura 65: Saturação no meio poroso heterogêneo em $\mathrm{T}=1$.

Figura 66: Saturação no meio poroso heterogêneo em T = 100. 
Figura 67: Saturação no meio poroso heterogêneo em $T=150$.

Figura 68: Saturação no meio poroso heterogêneo em $\mathrm{T}=200$. 147

Figura 69: Saturação no meio poroso heterogêneo em T = 250. 148

Figura 70: Geometria do modelo SPE 10 -Model 2.

Figura 71: Porosidade do meio heterogêneo (Formação "Tabert"). 164

Figura 72: Porosidade do meio heterogêneo (Formação "Upper Ness").

Figura 73: Variação de pressão no reservatório (Aproximadamente três meses depois)

Figura 74: Variação de pressão no reservatório (Aproximadamente um ano e nove meses depois)

Figura 75: Variação de pressão no reservatório (Aproximadamente cinco anos e seis meses depois)

Figura 76: Saturação da água no meio heterogêneo no layer 35

Figura 77: Saturação da água no meio heterogêneo do layer 35 (Três meses depois, aproximadamente)

Figura 78: Saturação da água no meio heterogêneo do layer 35 (Cinco anos depois, aproximadamente)

Figura 79: Saturação da água no meio heterogêneo do layer 50 (Três meses depois, aproximadamente)

Figura 80: Saturação da água no meio heterogêneo do layer 50 (Cinco anos depois, aproximadamente) 


\section{Lista de Símbolos}

$\varepsilon \quad$ Coeficiente de difusão

$\Delta \quad$ Operador Laplaciano

$\varphi \quad$ Função base radial

$\alpha \quad$ Fase do fluido

$\gamma_{l \alpha} \quad$ Fração volumétrica (fração de massa)

c Compressibilidade

$P_{w} \quad$ Pressão da água

$P_{o} \quad$ Pressão do óleo

$\vec{g} \quad$ Aceleração gravitacional

$U \quad$ Saturação do meio poroso

$\rho \quad$ Massa específica ou densidade

$\vec{V} \quad$ Campo de velocidade

$t \quad$ Tempo

A Área do corte transversal para o fluxo

$q$ Razão de massa de injeção por unidade de volume

$c_{r} \quad$ Compressibilidade da rocha

$\Phi$ Porosidade do meio

$P \quad$ Pressão do meio

$\vec{K} \quad$ Permeabilidade absoluta

$k r_{\alpha} \quad$ Permeabilidade relativa

$\mu_{\alpha} \quad$ Viscosidade da fase

$U_{r w} \quad$ Quantidade mínima de água presente no meio poroso

$U_{r o} \quad$ Quantidade mínima de óleo presente no meio poroso

$\phi \quad$ Campo escalar

$\Gamma \quad$ Curva de nível zero (ou zero Level Set)

$\xi \quad$ Nó, ponto do domínio ou ainda partícula. 
"A renúncia é o que nos humaniza" (Pe. Adroaldo sj) 


\section{1 Introdução}

Este capítulo apresenta as considerações gerais com relação à simulação numérica de modelos matemáticos de reservatórios de petróleo, os objetivos desta dissertação e os trabalhos relacionados com os objetivos propostos. No final, a estrutura da dissertação é apresentada.

\section{1.}

Considerações Gerais

A simulação numérica de modelos matemáticos de reservatórios de petróleo e a visualização de resultados têm sido muito utilizadas na indústria de petróleo. Esses modelos são constituídos por um conjunto de equações diferenciais parciais, que descrevem a variação temporal e espacial do fluxo dos fluidos nos reservatórios de petróleo (Peaceman, 1977), (Hasle et al, 2007). Junto a esses modelos são utilizadas diversas hipóteses relativas às condições iniciais e de fronteira. A finalidade da simulação numérica aplicada é de prever o desempenho futuro de um reservatório e encontrar formas e meios de aperfeiçoar a recuperação de petróleo antes de sua construção. Um reservatório geralmente é caracterizado pela natureza da rocha e dos fluidos; sendo assim, um reservatório é quase sempre heterogêneo. As propriedades dos reservatórios dependem muito da localização do espaço e do meio poroso em que se encontram. As propriedades da rocha podem drasticamente mudar de acordo com o meio como por exemplo a permeabilidade e a porosidade (Peaceman, 1977). Na simulação de reservatórios, o principal interesse é modelar o deslocamento do óleo, da água ou do gás dentro de um meio poroso (Rosa et al, 2006).

O objetivo principal desta pesquisa é estudar e analisar o deslocamento simultâneo dos dois fluidos imiscíveis, a água e o óleo, assumindo que não há transferência de massa entre os dois fluidos. Em um sistema bifásico óleo-água, a água na maioria das vezes é considerada a fase molhante e o óleo a fase não molhante do meio poroso. Esta pesquisa adotou este conceito como premissa, porém vários novos conceitos pertinentes ao escoamento multifásico devem ser 
detalhados no capítulo 2 (Peaceman, 1977), (Rosa et al, 2006), (Hasle et al, 2007).

Devido à complexidade dos sistemas lineares e não lineares dos modelos matemáticos em reservatórios de petróleo, métodos numéricos são propostos para discretização das equações diferencias parciais. Os métodos numéricos explícitos como, por exemplo, o método de Front Euler (Peaceman, 1977), exige sempre a utilização da condição Courant-Friedrichs-Lewy (CFL) (Peaceman, 1977), ou seja, a condição de estabilidade e de convergência do modelo numérico. Geralmente os métodos implícitos são estáveis como o método de Back Euler e os métodos de Crank-Nicolson (Hasle et al, 2007), que por isso demandam a solução de grandes sistemas de equações não lineares em cada passo de tempo. Por outro lado, os métodos explícitos são computacionalmente eficientes e considerados razoavelmente estáveis, devido à condição $C F L$ e demandam cálculos extremamente longos para simular um grande período de tempo em modelos de grande escala.

Os Métodos numéricos tradicionais, como diferenças finitas (na década de 1950), elementos finitos (na década de 1960), ou métodos de volumes finitos (na década de 2000), foram motivados principalmente por meio de simulações unidimensionais e bidimensionais para problemas de engenharia através de equações diferenciais parciais (Peaceman, 1977). A discretização desses métodos requer algum tipo de malha computacional, por exemplo, uma triangulação da região de interesse ou qualquer outro tipo de malha. A criação destas malhas torna-se uma tarefa bastante difícil, em três dimensões, e praticamente impossível para problemas de dimensão superior, entre outros motivos como a discretização da malha (Hasle et al, 2007). Por outro lado, os métodos meshfree (Wendland, 2005), (Fasshauer, 2007), baseados principalmente na norma da distância euclidiana entre os pontos e também de funções bases radiais, funcionam como se o problema fosse unidimensional, isto é, em vez de espaço euclidiano $x y z$ (se for em $R^{3}$, por exemplo), usa-se o espaço $r$ dado pela distância entre pontos do mesmo espaço euclidiano (Fasshauer, 2007).

Os métodos meshfree começaram a aparecer na literatura matemática na década de 1980. Estes métodos são de grande importância não só na matemática, mas também na comunidade de engenharia. A grande motivação dos métodos de aproximação meshfree vieram de aplicações geofísicas, mapeamentos e da 
meteorologia. Essas aplicações se baseiam principalmente em funções de base radial (Fasshauer, 2007) e nos métodos de mínimos quadrados (Gram, 1883), (De Forest, 1874). Mais tarde, aplicações de métodos meshfree apareceram em muitas outras áreas, tais como na solução numérica de equações diferenciais parciais, computação gráfica (principalmente em modelagem de terreno e reconstrução da superfície), em simulações de fluido, inteligência artificial, teoria de aprendizagem estatística, redes neurais, processamento de sinal e imagem, teoria da amostragem, estatística, finanças e otimizações (Fasshauer, 2007), (Wendland, 2005). Assim, grande parte do trabalho relacionado com métodos de aproximação meshfree é interdisciplinar, como se pode notar acima. Uma das razões para a utilização ampla de métodos meshfree é o fato de que representações de modelos sem a definição de malhas são muitas vezes mais adequadas para lidar com mudanças geométricas, formações de fraturas ou ocorrências de grandes deformações do domínio de interesse em comparação com as técnicas clássicas de modelos com malhas como diferenças finitas, elementos finitos ou volumes finitos.

Sucintamente, os métodos meshfree são métodos numéricos de resolução de equações diferencias parciais com condições de contornos ou iniciais que usam algum critério de aproximação de forma local, ou global, para obter soluções de valores pontuais (Fasshauer, 2007). Diferentemente dos métodos baseados em malhas, os métodos meshfree são caracterizados pelo uso de um conjunto de nós, espalhados pelo domínio do problema, sujeitos ou não a modificações adaptativas durante a simulação. A adaptação é feita baseada em regras e estratégias personalizadas para a modificação do nó, ou do conjunto de nós (Iske e Käser, 2005). Para este fim, prefere-se trabalhar com interpolação de dados esparsos e funções bases radiais $R B F$, interpolação de métodos baseados nas aproximações de $R B F$ Hermite e ainda aproximações por RBF Kansa (Fasshauer, 2007). Segundo Gregory Fasshauer, esses métodos fornecem uma reconstrução numericamente estável de arbitrária ordem de aproximação local a partir de dados esparsos. A formulação desses métodos é detalhada nas próximas seções. Por agora, faz-se importante apontar que a interpolação por funções de base radial $(R B F s)$ tornou-se uma ferramenta de notoriedade na teoria da aproximação multivariada de dados esparsos especialmente por permitir se trabalhar em dimensão arbitrária do espaço. Porém a definiçãa das condições de fronteira é um 
dos maiores problemas desses métodos por apresentarem pontos independentes sem conexão de fronteira.

Existe uma grande diversidade de métodos meshfree, tais como, Smoothed Particle Hydrodynamics (SPH), Reproducing Kernel Particle Method (RKPM); Particle-Partition of Unity Methods (PPUM); Moving Point Methods; SemiLagrangian method (SLM); Method of Characteristics; e Particle Methods for the Boltzmann equation. Para mais informações sobre o tema, algumas referências podem ser encontradas em Wendland (2005) e Fasshauer (2007).

O esquema Semi-Lagrangeano ou caraterístico é um método numérico de solução de sistemas de equações diferenciais parciais, lineares e não lineares para descrever o processo de advecção. O método está sendo amplamente utilizado em modelos de previsão numérica de tempo para integração das equações que governam o movimento atmosférico (Kalnay, 2003). O mesmo método passou a ser aplicado a problemas de visualização e simulação na área de dinâmica dos fluidos computacionais e em computação gráfica, (Iske e Käser, 2005) e esses métodos são adequados para problemas dominados por advecção que se encaixa neste contexto de métodos meshfree. O esquema Semi-Lagrangeano se baseia na combinação dos métodos Eulerianos e Lagrangeanos e ainda é caracterizado por grandes passos de tempo, captura de frentes de onda e conservação de massa.

Sendo assim, após uma breve descrição sobre alguns métodos numéricos, esta pesquisa optou por utilizar o método característico ou Semi-Lagrangeano para modelar numericamente os problemas visuais adaptativos de reservatórios propostos. Neste contexto, este trabalho pretende apresentar uma solução eficiente através da combinação do método Semi-Lagrangeano adaptativo com interpolação local meshfree usando splines poliharmônicas como funções de base radial. Adicionalmente, pretende-se melhorar e estender o método adaptativo meshfree proposto por Iske e Käser (2005), tanto em 2D como em 3D. Além disso, considera-se um meio poroso heterogêneo, propondo uma nova formulação para a mudança nas propriedades da região interna do meio poroso. 


\section{2.}

Objetivo

O objetivo desta pesquisa é propor um método meshfree adaptativo de advecção para problemas de fluxo bifásico de fluidos incompressíveis e imiscíveis em meios porosos heterogêneos tridimensionais. Este método se baseia principalmente na combinação do método Semi-Lagrangeano adaptativo com interpolação local meshfree usando splines poliharmônicas como funções de base radial. O método proposto é uma melhoria e uma extensão do método adaptativo meshfree AMMoC proposto por Iske e Käser (2005) para modelagem $2 D$ de reservatórios de petróleo. Inicialmente este trabalho propõe um modelo em duas dimensões, contribuindo com uma melhoria significativa no cálculo do Laplaciano, utilizando os métodos meshfree de Hermite e Kansa. Depois, o método é ampliado para três dimensões ( $3 D)$ e para um meio poroso heterogêneo. O método proposto é testado com o problema de five spot e os resultados são comparados com os obtidos por sistemas bem conhecidos na indústria de petróleo.

\section{3 . Trabalhos Relacionados}

No melhor do conhecimento do presente autor, não há trabalhos na literatura que considerem conjuntamente todas as características propostas nesta tese, ou seja, um método meshfree adaptativo de advecção para problemas de fluxo bifásico de fluidos incompressíveis e imiscíveis em meios porosos heterogêneos tridimensionais. O método aqui proposto é uma melhoria e uma expansão para $3 D$ do trabalho de Iske e Käser (2005), cujos detalhes estão detalhados ao longo do texto. Portanto, esta seção apresenta apenas os trabalhos diretamente relacionados ao método proposto: Métodos Level Set, Semi-Lagrangeano e Meshfree.

\subsubsection{Trabalhos relacionados referentes ao método Level Set}

O método Level Set foi desenvolvido em 1988 por Osher e Sethian como método simples para calcular e analisar o movimento de uma interface no espaço sob um campo de velocidade. Esta velocidade depende da posição, do tempo e da 
geometria da interface. A seguir são apresentados alguns trabalhos mais relevantes sobre o método Level Set.

Guedelman et al (2005) desenvolveram um novo método computacional de simulação de partículas para acoplamento de fluxos incompressíveis, tais como sólidos e líquidos, para o tratamento de sólidos finos que não contêm uma região interior. O trabalho foi modelado baseado na triangularização da superfície utilizando o método Level Set para o deslocamento de superfícies. O método Ray Tracing foi utilizado para aumentar o número de interpolações. Ademais, técnicas como diferenças finitas e renderizacão foram utilizadas para que não haja vazamento entre as superfícies triangularizadas. O exemplo da Figura 1 ilustra que o método é eficiente para sólidos/líquidos e também para fumaça, especialmente onde se produz interações entre as partículas. O método Level Set foi utilizado para o tratamento da interface e também trabalha com ambas as superfícies rígidas e deformáveis triangularizadas.

Ainda no exemplo da Figura 1 do trabalho de Guedelman et al. (2005), é possível observar uma superfície opaca representado por uma grade de simulação de fluidos e gerado pelo método Level Set.

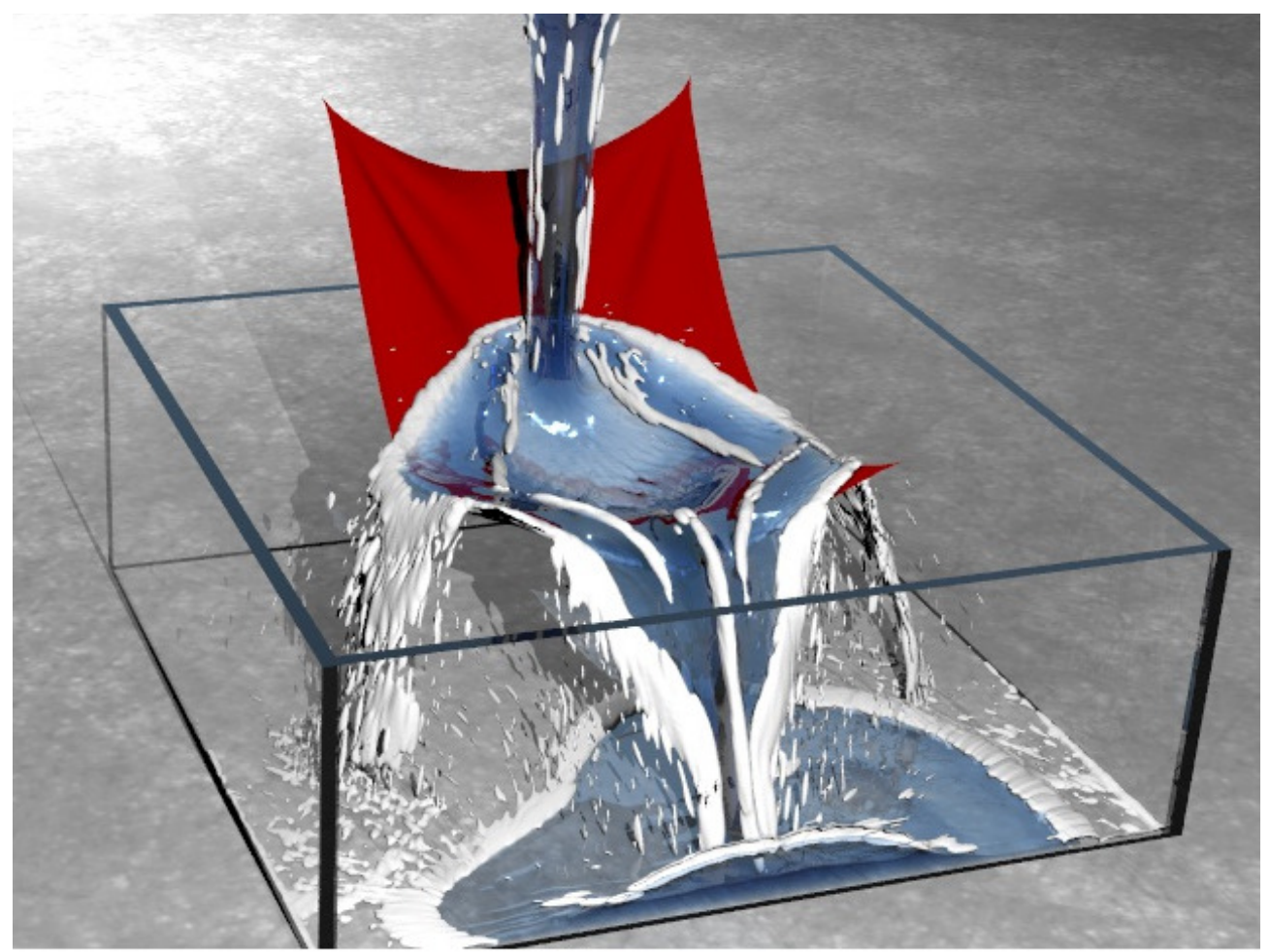

Figura 1: Superfície Opaca Gerado pelo Método Level Set (Guedelman et al, 2005) 
Losasso et al. (2006) utilizaram o método Level Set para simulação de duas regiões distintas (no primeiro caso utilizaram água e ar e, no segundo caso, combustível e outros produtos). O foco principal deste trabalho foi estender o método para fins de simulação de diversas regiões que podem ser líquidos (ou gases) de qualquer tipo com diferentes viscosidades, densidades, propriedades viscoelásticas, entre outros. Propuseram também técnicas para interação entre materiais avaliando a tensão superficial, reações químicas e combinação de dois materiais para formar um terceiro. O método Level Set foi utilizado separadamente para definir cada região e assim propuseram um novo algoritmo de projeção que será um vetor resultante de todos os valores do método Level Set em um único. Os pesquisadores encontraram dificuldades na discretização da interpolação no método Semi-Lagrangeano e no método Ray Tracing e foi observada perda de volume pelo novo método Level Set como no método original. A Figura 2 exemplifica que a maior parte de perda de massa ocorre longe dos pontos tridimensionais, regiões onde o novo método é idêntico ao método Level Set original. Na conclusão da pesquisa encontram-se indicações que isto pode ser resolvido aumentando o número de partículas do método Level Set com o método "Adaptative Octree" (Losasso et al, 2004).
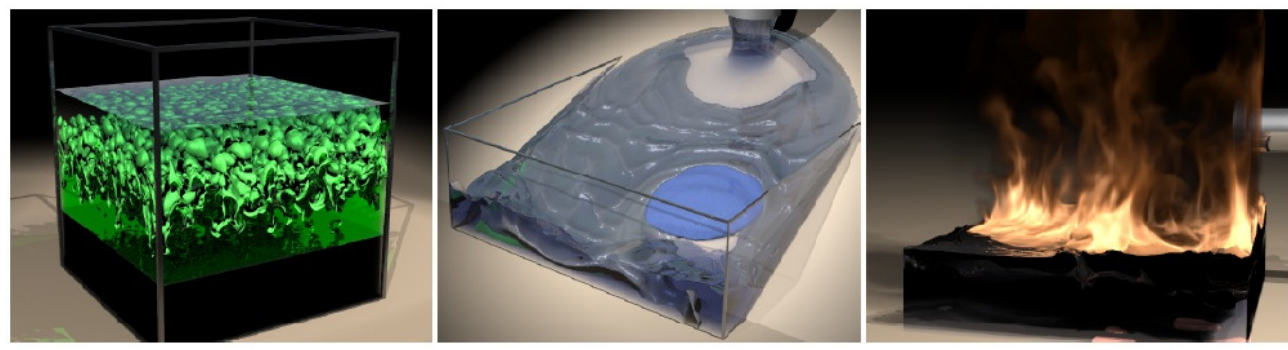

Figura 2: Rayleigh - Taylor, 4 e 5 fases (Losasso et al, 2006)

A Figura 2, com imagens extraídas de trabalho de Losasso et al. (2006), agrupa três simulações: instabilidade de Rayleigh - Taylor, 4 fases (esquerda); líquidos de viscosidade variável, 5 fases (centro); e queima de óleo em água, 4 fases (direita).

Ainda no ano 2006, Geiger et al (2006) fizeram uma análise fotorealista das águas do oceano interagindo com objetos gerados por computador (Figura 3). A 
finalidade deste trabalho era a simulação de efeitos especiais do filme "Poseidon" para formação de ondas utilizando o método Level Set, o qual permite o deslocamento de superfícies e de volumes internos. Sendo a onda uma interface entre dois fluidos, o ar e a água, a interface foi feita por uma malha de triângulos, renderizando múltiplas partículas secundárias - processo fisicamente baseado em simulação de fluido. Um dos grandes desafios desse projeto foi a criação da água em grande escala.

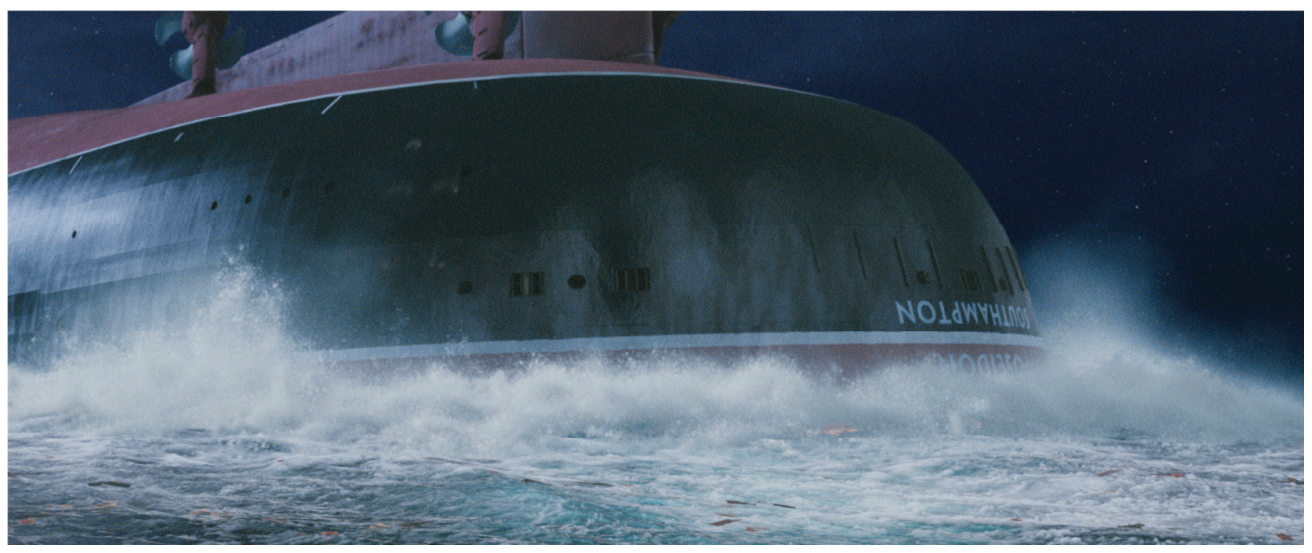

Figura 3: Rayleigh - Formação de Onda (Geiger et al, 2006)

Vorgelegt Von (2013) estudou o método de Level Set para a captura de interfaces com aplicações em problemas de fluxo bifásico. Naquela pesquisa, o autor teve como principal objetivo a comparação entre o método StreamlineUpwind-Petrov-Galerkin (SUPG) e o método de Galerkin Descontínuo (DG) com o método de fluxo Upwind. Ele discretizou o método de Level Set utilizando elementos finitos. O método SUPG se baseia no método de elementos finitos contínuo e estabilizado. O método $D G$, também, se baseia no método de elementos finitos sendo que as funções de elementos finitos são permitidas serem descontínuas nas fronteiras dos elementos. O método de $D G$ junto com o método de Upwind foram utilizados pelo autor para discretização espacial e o método de Crank-Nicolson para discretização temporal. O autor apresentou alguns resultados em diferentes instantes de tempo. Seguem abaixo alguns dos resultados do trabalho supracitado. 


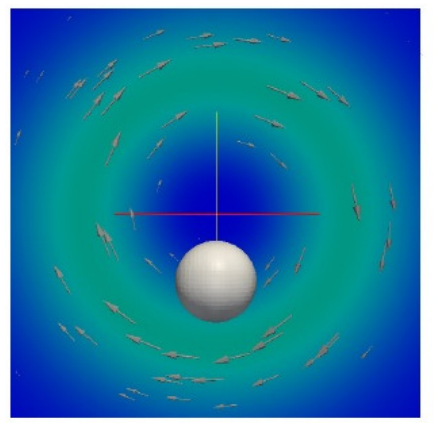

(a) SUPG2, $t=0$

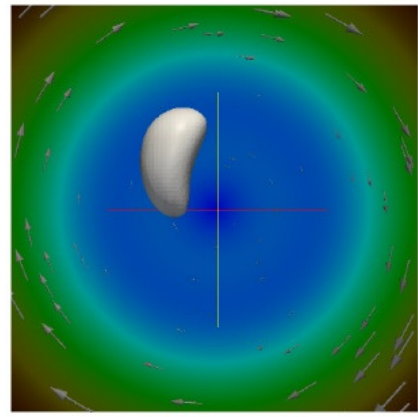

(c) SUPG2, $t>3 / 4 T$

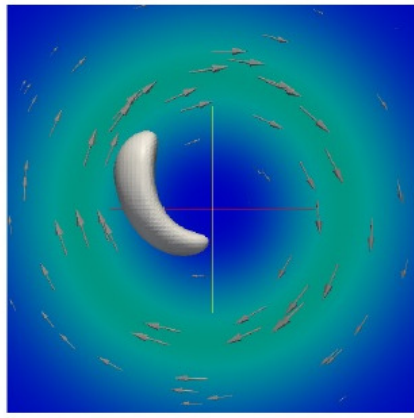

(b) SUPG2, $t=T / 2$

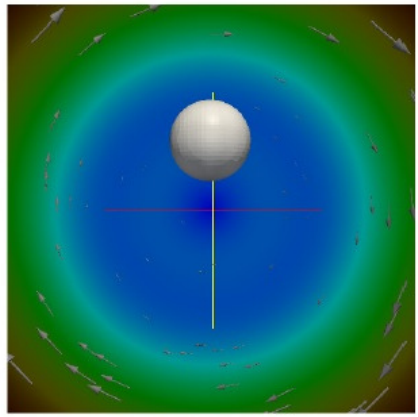

(d) SUPG2, $t=T$

Figura 4: Interface e Velocidade de Campo para Diferentes Instantes De Tempo (Vorgelegt Von, 2013)

A Figura 4 representa a deformação e rotação de uma esfera através de um campo de velocidade angular para um instante de tempo $t$, obtidos por Vorgelegt Von (2013). As cores subjacentes representam a magnitude e as setas indicam a direção do campo de velocidade do mesmo.

Patkar et al (2013) apresentaram um método híbrido LagrangeanoEuleriano para simulação dinâmica de bolhas, onde as bolhas podem crescer ou diminuir de volume, de acordo com as forças de pressão no fluido circundante. Segundo os autores, pequenas bolhas numericamente sub-resolvidas são evoluídas utilizando partículas lagrangeanas que são monoliticamente bidirecionais e acopladas ao fluxo circundante de uma maneira que se aproximem da frequência de oscilação da bolha, de acordo com a equação de Rayleigh-Plesset. As bolhas maiores foram modeladas pela abordagem tradicional do método de Level Set junto com o método Euleriano. Os autores apresentaram um exemplo de forma mais realística, ao simular o fluido interagindo com objetos, tal como ilustrado na Figura 5, onde o fluido interage com as hélices de um navio. 

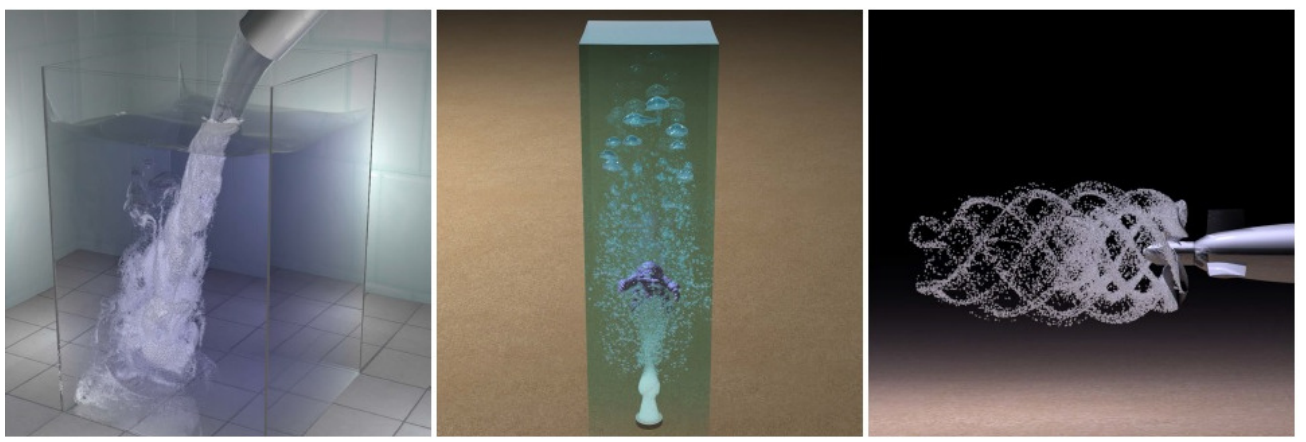

Figura 5: (Esquerda) a geração de torneira de bolhas através de retenção de ar; (Centro) Uma fonte para gerar pequenas bolhas que se fundem, interagem e crescem com o movimento do objeto; (Direita) Uma hélice gerando um helicoidal. (Patkar et al, 2013)

Gjennestad e Munkejord (2015) propuseram modelos matemáticos para o transporte de calor e para a transferência de massa entre as fases (liquido e gás) devido à vaporização e condensação. Segundo os autores, esses modelos têm sido implementados em fluxo bifásico baseado no método de Level Set. Em algumas simulações, os autores comparam e analisam a convergência das soluções analíticas com as correspondentes soluções simuladas numericamente. Eles também simularam um caso tridimensional, fisicamente mais realista, e rotacionalmente simétrico em torno de um eixo. Neste exemplo, uma gota de metano líquido é vaporizada e os autores mostram que o modelo produz resultados que são consistentes com as expectativas qualitativas, como se pode observar na Figura 6. 


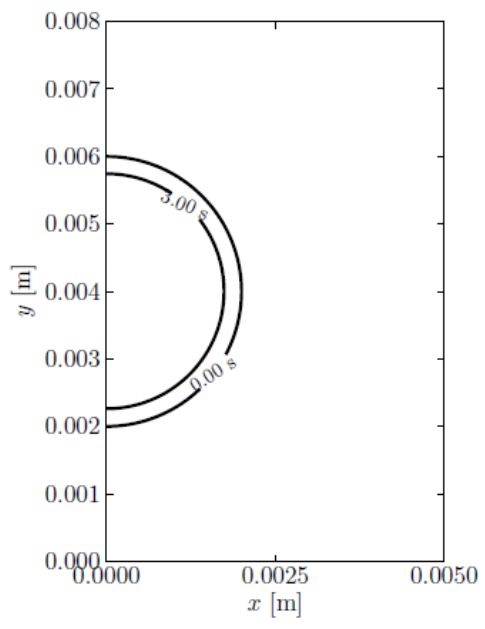

(a)

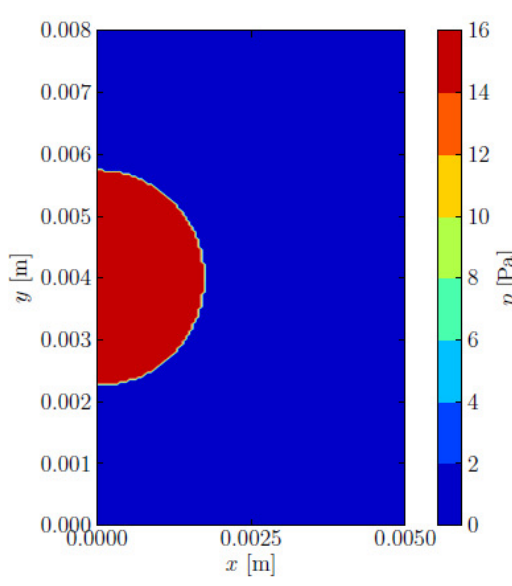

(c)

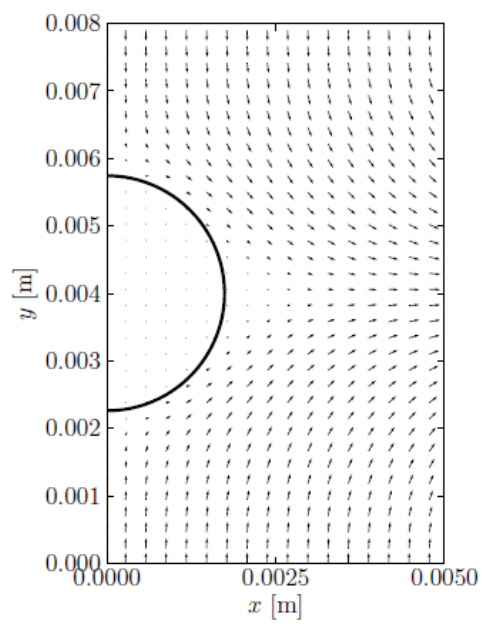

(b)

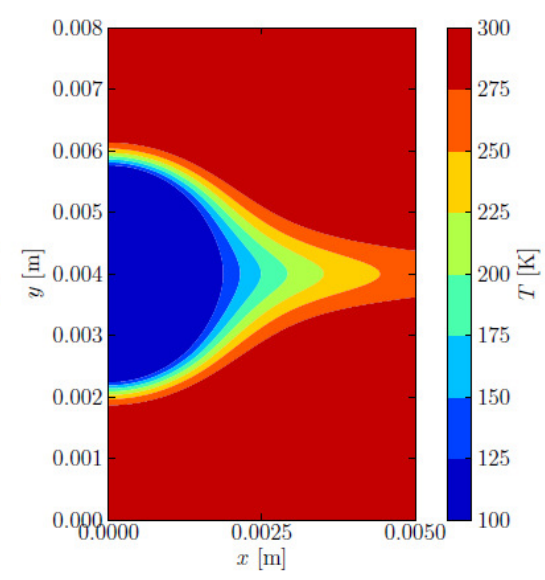

(d)

Figura 6: Propagação do fluido gás através da vaporização, dada pelo campo de pressão e pelo campo de temperatura (Gjennestad e Munkejord, 2015)

Na Figura 6(a) se nota o encolhimento da gota devido à vaporização e, na Figura 6(b), observa-se que o gás está fluindo e se misturando com o gás vaporizado. O campo de pressão é apresentado na Figura 6(c), onde se constata que não foram considerados os efeitos gravitacionais, isto é, a pressão é quase uniforme dentro de cada fase (liquido ou gás). O campo de temperatura é exibido na Figura 6(d), onde o calor é advectado com o fluxo de fluido na direção da vaporização. 


\subsection{2.}

Trabalhos relacionados referentes ao método Semi-Lagrangeano

O método Semi-Lagrangeano foi primeiramente utilizado em sistemas de Convecção - Difusão com o objetivo de alcançar duas importantes características: passo de tempo grande e estabilidade. A aproximação Semi-Lagrangeana tem sido utilizada em meteorologia para predições numéricas das condições climáticas, no qual o uso de grandes passos de tempo é essencial (Kalnay, 2003). Wiin (1959) no final dos anos 1950 e Krishnamurti e Sawyer (1960) no início dos anos 1960 foram pioneiros ao empregarem o método Semi-Lagrangeano; entretanto, foi somente após os trabalhos de Robert (1972 e 1981), que o método Semi-Lagrangeano passou a ser largamente utilizado em problemas advectivos (Stanfort Cotê, 1991).

A combinação que métodos tem sido utilizada recentemente em modelagens de reservatório de petróleo (Iske e Käser, 2005), onde se propõe um novo método de partículas Meshfree para o modelo da equação de Buckley-Leverett. O esquema utilizado Meshfree Advection, chamado de AMMoC (Adaptive Meshfree Method of Characteristics), é um método característico que combina um método SemiLagrangeano adaptativo com interpolação local Meshfree usando splines poliharmônicas. Neste trabalho, o método $A M M o C$ é aplicado aos problemas de simulação de reservatório de petróleo conhecidos como five spot. Segundo Iske e Käser, os resultados numéricos apresentados em sua pesquisa foram comparados com dois simuladores comerciais de reservatórios de petróleo, o Eclipse e o FrontSim, e o esquema de advecção Meshfree AMMoC apresentou um bom desempenho.

Berhens et al (2002) propuseram uma combinação do método SemiLagrangeano adaptativo com interpolação local usando função de base radial para suavizar as superfícies, que é conhecido como método Backward Characteristics, - sendo um novo esquema de advecção adaptativo Meshfree para resolver numericamente as equações lineares e não lineares de transporte. Neste artigo, o método não linear é estendido para equações de transporte. O bom desempenho do método resultante é mostrado nos exemplos numéricos em dois problemas específicos não-lineares dos modelos: equação de Burgers e a equação de Buckley-Leverett que descrevem um fluxo de fluido bifásico em um meio poroso. 
Lentine et al (2012) propuseram um novo método de simulação e visualização de fluxos incompressíveis de superfície livre que permite grandes intervalos de tempo da ordem de 10-40 vezes maior do que o determinado pela condição de Courant-Friedrichs-Lewy. Essa é uma das principais vantagens do método de advecção Semi-Lagrangeana além de sua estabilidade. O estudo comprova que pode ser considerado tanto um fluido gás (fumaça), bem como um fluido liquido (água).

A grande diferença entre os fluidos é que no fluido em forma de fumaça o campo de velocidade é definido globalmente e já o fluido em forma de água precisa se mover de uma maneira não influenciada pelo fluxo de ar ao redor; e esta relação causa pequenos problemas no campo de velocidade. No mesmo estudo de Lentine et al (2012), isso foi resolvido através da extrapolação do campo de velocidade onde a perda de massa foi solucionada pelo uso do modelo de advecção conservativa junto com o método de advecção Semi-Lagrangeana. O método Level Set de partículas também foi utilizado acoplado com o método de advecção Semi-Lagrangeano. No fim do trabalho os autores acima citados identificaram que o método de Level Set de Partículas falha quando há grandes passos de tempo. A Figura 7 exibe um dos principais resultados adquiridos pelos autores.

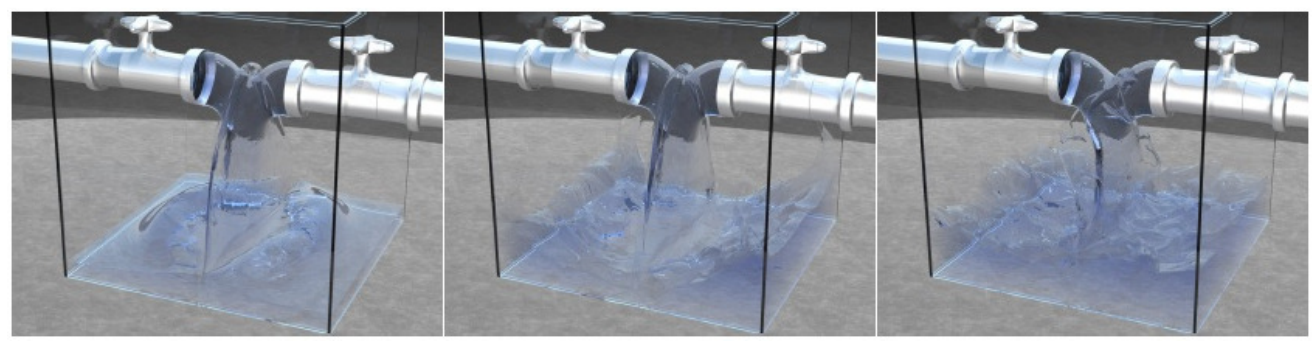

Figura 7: Derramamento de água em uma caixa a partir de duas fontes de fluxo opostas (Lentine et al, 2012)

A Figura 7 ilustra o derramamento de água em uma caixa, a partir de duas fontes de fluxo opostas uma na frente da outra. Durante a simulação, as fontes foram primeiramente ativadas até que a água atingisse o fundo do recipiente, utilizando um passo de tempo maior do que normalmente é proposto pela condição $C F L$. 
English et al (2013) introduziram um novo método para a simulação do fluido água em grande escala. A grade utilizada foi discretizada com a sobreposição de grades cartesianas através da translação e da rotação, a fim de decompor o domínio em diferentes regiões de interesse com diferentes resoluções espaciais. A finalidade dessa grade sugerida pelos autores é para acompanhar o movimento tanto das características dos fluidos como também dos objetos sólidos, permitindo, assim, a adaptabilidade espacial dinâmica do domínio. Estes mesmos autores resolveram um problema de fluxo incompressível e viscoso utilizando as equações de Navier Stokes baseado nas estratégicas de discretização LagrangianEulerian por meio do esquema de advecção Semi-Lagrangeana. Os autores modificaram o método Level Set de partículas para adaptação das grades, incluindo o tratamento de partículas nas fronteiras. A malha adaptativa foi feita através de uma malha por inserção de pontos de Voronoi local, para resolver principalmente o campo de pressão e velocidade e também problemas que normalmente surgem na vizinhança da interface. A Figura 8 ilustra alguns dos resultados obtidos por English et al. (2013).

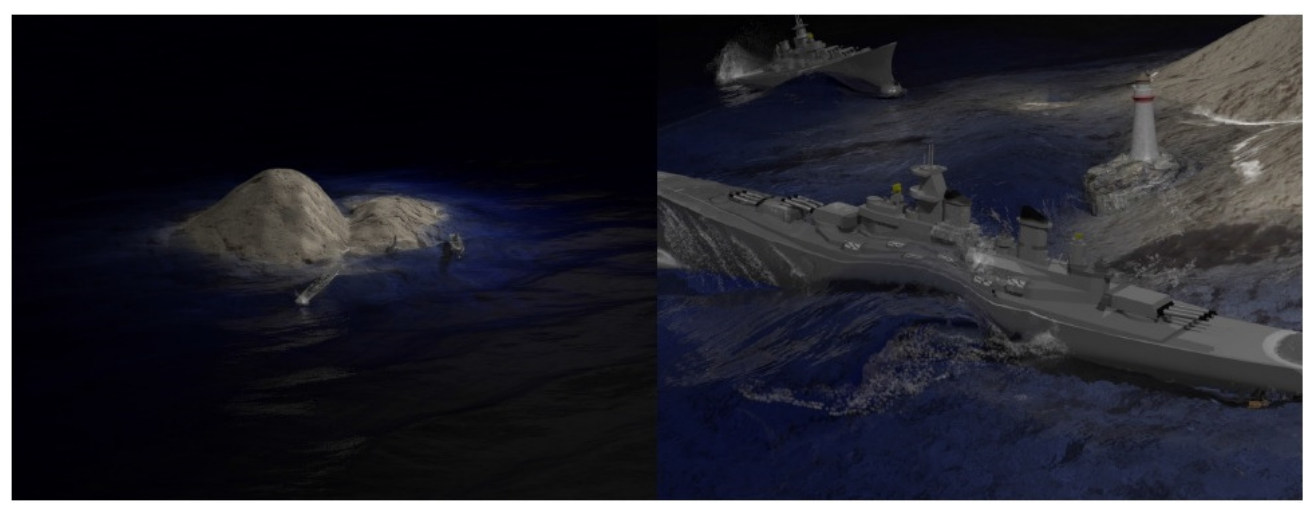

Figura 8: Dois navios em tempestade próxima de uma ilha (English et al, 2013)

A Figura 8 exemplifica dois navios se movendo de forma totalmente instável durante uma tempestade. Os autores mencionaram que perto dos navios houve um refinamento, colocando grades nos objetos, o que facilitou uma maior interação entre o líquido e o sólido. 


\subsection{3.}

Trabalhos relacionados referentes aos métodos meshfree.

Ultimamente os métodos meshfree têm sido muito utilizados na comunidade científica e vêm ganhando uma maior relevância por serem métodos interdisciplinares e se adequarem a situações complexas, com grandes deformações e liberdade para mudanças na geometria do domínio de interesse. Inicialmente os métodos meshfree foram aplicados em problemas de geodesia, geofísica, mapeamento, ou meteorologia. Depois, estes métodos passaram a ser usados em muitas outras áreas, tais como: solução numérica de EDPs em muitas aplicações de engenharia, computação gráfica, ótica, inteligência artificial, estatística, processamento de sinal e de imagem, teoria da amostragem, estatística e otimização. A seguir é apresentada uma breve história dos métodos meshfree.

Lucy e al, (1977) desenvolveram o método SPH (Smoothed Particle Hydrodynamics). Inicialmente o método foi desenvolvido para modelagem e simulação de problemas astrofísicos, sendo posteriormente estendidos para aplicações em mecânica dos fluidos. Portanto, o método $S P H$ pode ser aplicado a uma grande variedade de problemas. Muitos estudos foram focados em extensões e melhoramentos importantes do método original. Os aspectos numéricos têm se tornado cada vez mais robustos uma vez que algumas técnicas foram modificadas e a correção de alguns métodos foram propostas. Em seguida, Liska e Orkisz (1980) propuseram um método de diferenças finitas generalizada que pode lidar com grades irregulares e arbitrárias. Nayroles et al. (1992) foi o primeiro a usar aproximações por mínimos quadrados em um método de Galerkin para formular o chamado método dos elementos difusos (DEM). Belytschko et al. (1994), com base no $D E M$, estenderam a ideia para dar origem a um novo método meshfree, conhecido até o momento como Element-Free Galerkin Method (EFG). O EFG é atualmente um dos métodos meshfree mais populares, com muitas aplicações em problemas principalmente na área de mecânica dos sólidos. Posteriormente algumas outras extensões ao método aconteceram nos anos de 1996, como a proposta de Krysl e Belytschko (1996a).

Após isso, Atluri e Zhu (1998) propuseram um novo método de meshfree, o Meshless Local Petrov-Galerkin Method (MLPG), que tem sido aplicado à análise das estruturas de viga (Atluri et al., 1999), (Liu e Gu, 2000) e (Atluri e Shen 
2002). Logo depois, Lin e Atluri (2001) e Wu e Liu (2003) aplicaram o MLPG a problemas de fluxos de fluidos e em outros problemas mecânicos. Para resolver os problemas de fronteira gerados pelo método $S P H$, Li e Liu (2002) propuserem o método Reproducing Kernel Particle Method (RKPM).

Dentre os vários métodos meshfree pode-se destacar o método Moving Least Squares (MLS), (Piotr et al, 2005), que reconstrói funções contínuas a partir de um conjunto de amostras de pontos aleatórios usando mínimos quadrados. Um outro método relevante é o Hp-clouds proposto por Duarte e Oden (1996), que é aplicável aos domínios arbitrários e emprega um conjunto disperso de nós para construir soluções aproximadas para problemas de valor de fronteira BVPS.

Losasso et al. (2008), propuseram um novo método de acoplamento que combina dois métodos de simulação de partículas que são o método $S P H$ original, para simulação das partículas difusas, e o método Level Set para simulação das partículas densas, com a finalidade de simular misturas entre partículas. A estratégia destes autores é criar instrumentos de controle sobre as partículas que pertencem a regiões densas e aquelas que pertencem a regiões difusas. Um destes instrumentos de controle é o conceito de "partícula slip", que é uma partícula cuja mudança de velocidade pode ser controlada. Por outro lado, no método Level Set de partículas, partículas podem passar de um lado para outro da interface, requerendo ajustes. Se estas partículas cruzam para muito distante da interface, elas podem ser removidas do controle do Level Set e usadas para representar sprays ou borbulhas (dependendo se são água ou ar removidos, respectivamente). Estas partículas são, então, usadas como sementes do algoritmo SPH. Mostraram também que o novo método $S P H$ é eficiente, demonstrando vários exemplos, como o movimento das ondas do mar nas proximidades de um farol. (Figura 9). Umas das limitações do método é que a densidade de partícula não é válida nos vizinhos da interface entre ar e líquido, pois, o método $S P H$ não tem vizinhos em todos os lados. 

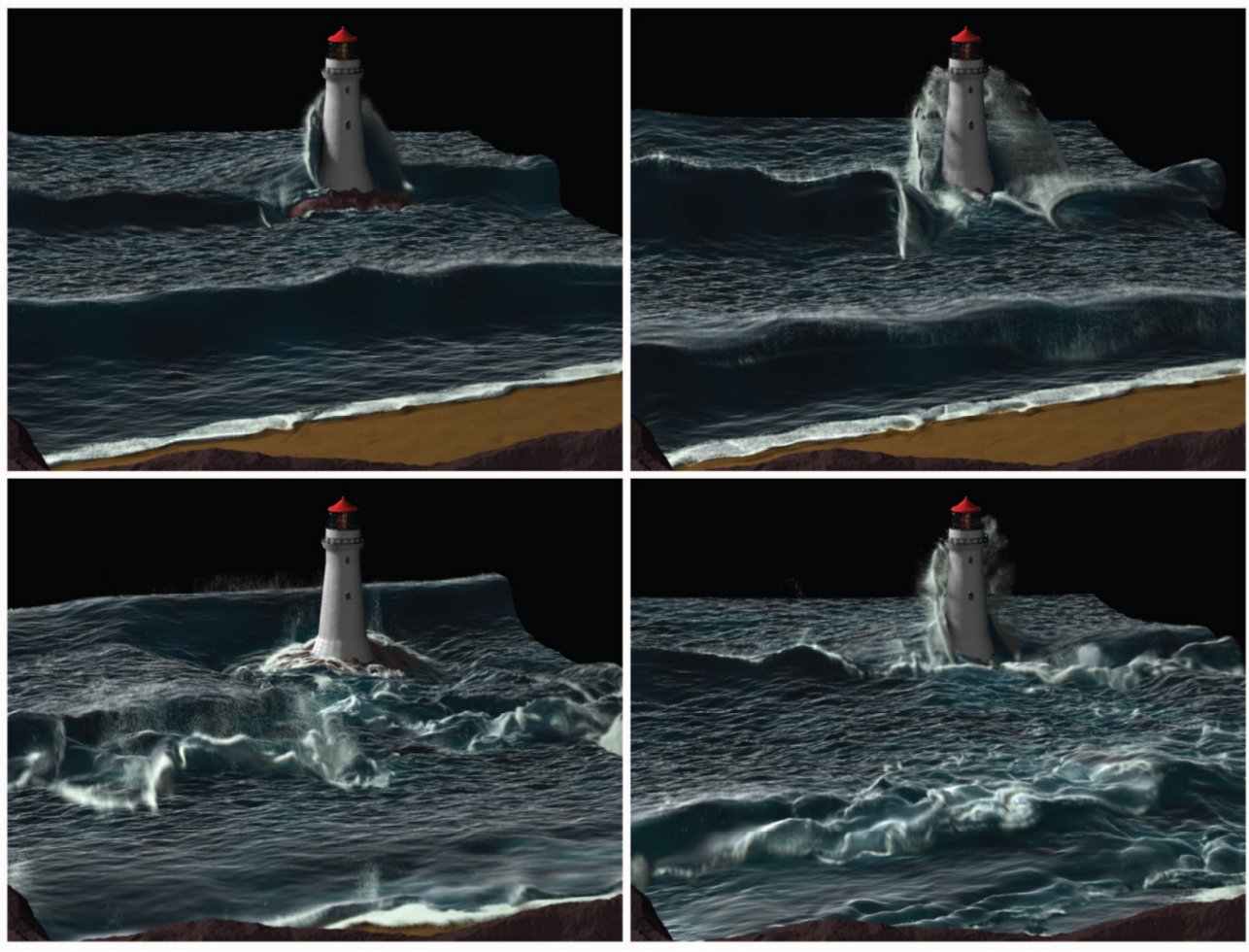

Figura 9: Uma grande cena de oceano simulado com o método two-way coupling entre os métodos de partículas SPH e o Level Set. Uma simulação de ar secundária também é usada para gerar uma segunda camada de detalhes com espuma e neblina provenientes de partículas do método SPH (Losasso et al, 2008)

Akinci et al (2012) propuseram uma forma de acoplamento do método de conservação dinâmica de fluidos $S P H$ e corpos rígidos arbitrárias baseadas em forças hidrodinâmicas. De acordo com os autores, as amostras aproximem da superfície de corpos rígidos com partículas de fronteira que interagem com o fluido, evitando problemas de deficiência, ou seja, descontinuidades espaciais e/ou temporais. O problema de fronteira da amostragem não homogênea é resolvido considerando uma contribuição relativa da partícula de fronteira a uma quantidade física. Isto facilita não só o processo de inicialização, mas também permite a simulação de múltiplos objetos dinamicamente. Os autores integraram o método de partículas nos métodos Weakly Compressible SPH (WCSPH) e Predictive-Corrective Incompressible SPH (PCISPH), e demonstraram a sua estabilidade e flexibilidade com vários cenários, incluindo o fluxo multifásico. 

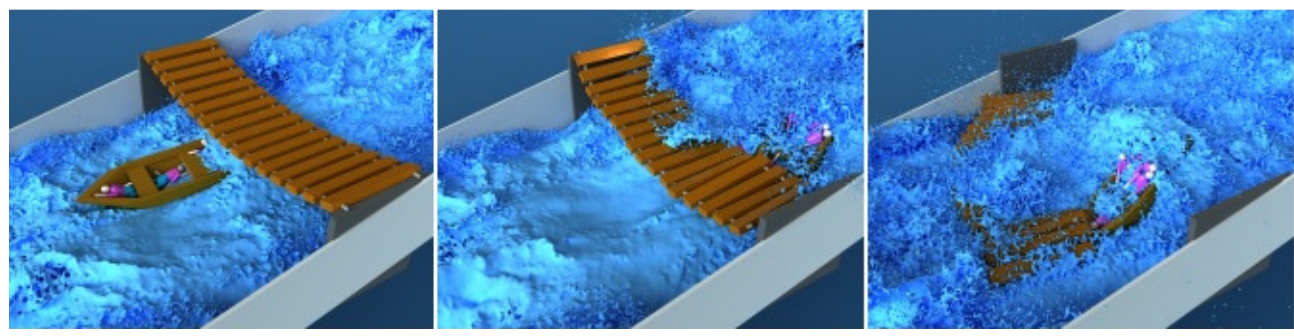

Figura 10: Interação fluido-objeto. Um barco passando por baixo da ponte (Esquerda). Um segundo barco colidindo com a ponte (meio e direita. (Akinci et al, 2012)

A Figura 10 exemplifica uma animação de uma grande interação de um objeto com um fluido. Nesta figura, um barco navega com uma figura humana (esquerda) e um segundo barco colide com a ponte devido a um aumento da vazão (centro e direita).

Chentanez et al. (2014) estudaram um novo método para simulação de fenômenos de grande escala através da combinação de partículas de água, com um objeto se movendo dinamicamente para a atmosfera e emergindo novamente nas partículas de água. Estes autores simularam também efeitos secundários especiais, tais como espumas, sprays e borbulhas. A simulação foi feita combinando a ideia de partículas com uma grade cartesiana em três dimensões. Para um melhor acoplamento entre as partículas e a grade cartesiana, os autores utilizaram a ideia de rastreamento da superfície da água com um campo de densidade junto com as estratégias baseadas em grade (grid). Para cenas ao ar livre, os autores simularam o exterior de água do domínio da grade $3 D$, resolvendo equações diferencias de águas rasas em um campo que envolve a altura. $\mathrm{O}$ método proposto pelos autores mostrou eficácia em vários cenários exibidos, incluindo o salto de uma baleia, todos em tempo real (Figura 11).
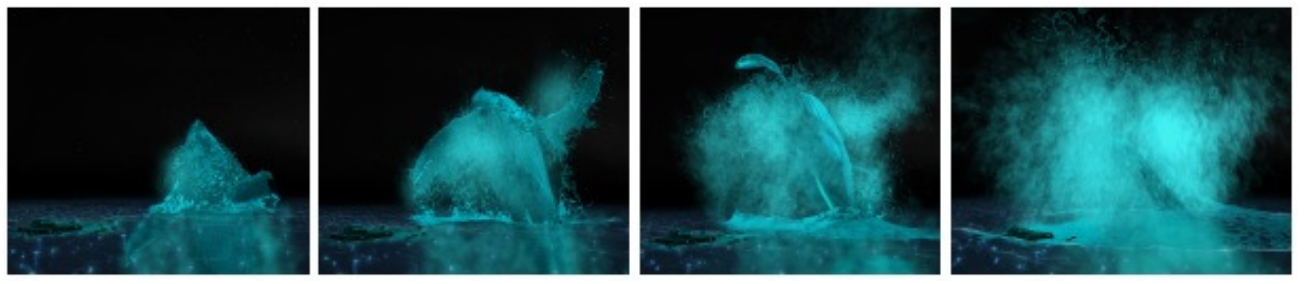

Figura 11: Interação de partículas da água com a baleia causando efeitos secundários como espuma, spray e borbulhas. (Chentanez et al., 2014) 
É importante também mencionar os métodos de ponto material (MPM Material Point Method), que são extensões do método PIC (particle-in-cell), usado em dinâmica dos fluidos, para a dinâmica de sólidos. Stomakhin et al. (2014) apresentaram um MPM para o transporte de calor, derretimento e solidificação de materiais. Isto permite uma maior variedade de comportamento de materiais em relação aos já flexíveis métodos $M P M$. O método proposto foi estendido pelos autores introduzindo um modelo constitutivo no tratamento implícito da evolução Euleriana que pode ser usado para simular materiais arbitrariamente incompressíveis. A Figura 12 ilustra a solidificação da lava, onde pode-se notar a lava emitindo luz de acordo com o espectro de corpo negro correspondente à temperatura simulada.

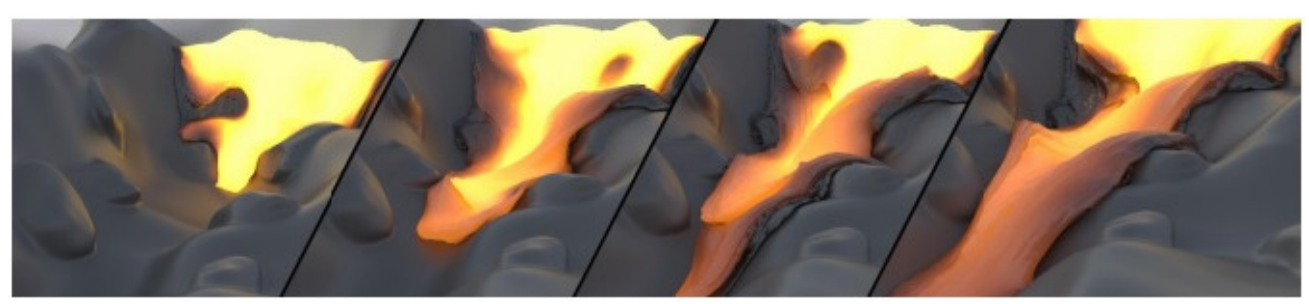

Figura 12: Solidificação da lava (Somakhin et al., 2014)

\section{4 . \\ Estrutura da Tese}

Os demais capítulos da tese estão organizados da seguinte forma. O Capítulo 2 aborda os conceitos fundamentais tais como: leis de conservação (massa, quantidade de movimento e energia), lei de Darcy, propriedades da rocha como a porosidade e a permeabilidade absoluta, modelo de Brooks-Corey e de Van Genuchten, derivada material, advecção, abordagens Euleriana e Lagrangeana e ainda o conceito de Level Set. O método de Level Set é uma ferramenta dinâmica de superfícies implícitas que simula e analisa movimentos de curvas, superfícies ou volumes.

No Capítulo 3 é discutida a formulação do método Semi-Lagrangeano presente no método Level Set para acompanhamento de interfaces móveis entre 
dois fluidos (bifásico). O método será utilizado para problemas de advecção como transporte de um campo escalar.

No capítulo 4, apresenta a formulação dos métodos meshfree, como o método de Kansa e o método de Hermite para aproximação das equações diferenciais parciais.

No capítulo 5 é apresentado um novo modelo multifásico de fluidos incompressíveis e imiscíveis em meios porosos heterogêneos tridimensionais.

No capítulo 6 apresenta os resultados obtidos nesta pesquisa.

Por fim, no capítulo 7 é apresentado as conclusões e trabalho futuros encontrado nessa pesquisa.

No Apêndice $A$ é apresentado um caso real do problema de five spot um Software comercial na área de dinâmica dos fluidos computacional conhecido por IMEX. 


\section{Conceitos Fundamentais}

Neste capítulo serão apresentadas as equações governantes que modelam o movimento do fluido num meio poroso heterogêneo, bem como uma breve introdução aos conceitos essenciais para formação dos métodos numéricos tanto baseados em grid como também em meshfree.

As equações governantes e as propriedades que podem ser utilizadas são as seguintes: equação de continuidade ou lei de conservação de massa, lei de Darcy, propriedades da rocha como a porosidade e a permeabilidade absoluta, modelo de Brooks-Corey e de Van Genuchten, a equação de quantidade de movimento, a equação de energia, derivada material, advecção e abordagens Euleriana e Lagrangeana. É válido mencionarmos que esses conceitos fundamentais estão intimamente associados aos métodos Level Set, Semi-Lagrangeano e métodos meshfree, utilizando um meio poroso heterogêneo. Esses conceitos serão discutidos com mais detalhes nos próximos capítulos. Neste capítulo objetiva-se abordar sucintamente os temas mencionados acima.

\section{1. Leis de Conservação}

As leis de conservação correspondem a uma classe de equações diferenciais parciais, que permite a compreensão de fenômenos da natureza físicomatemático tratada na engenharia.

\subsection{1.}

\section{Lei de Conservação de Massa}

A lei de conservação de massa diz que, em um sistema fluido, a massa não pode ser criada e nem pode desaparecer, ou seja, nunca se cria nem se elimina matéria, apenas é possível transformá-la de uma forma em outra (Fox e Mc 
Donald, 2001). A conservação de massa declara que, simplesmente, a massa em um sistema é constante, isto é,

$$
\left.\frac{d \text { Massa }}{d t}\right)_{\text {Sistema }}=0
$$

Uma vez que a massa seja conservada, se pode dizer que:

\section{Razão de Entrada - Razão Saída + Razão Injetada = Razão Acumulada.}

Isto é, para um caso unidimensional se pode dizer que,

$$
\begin{aligned}
& \left(A \rho \vec{V}_{x}\right)_{x}-\left(A \rho \vec{V}_{x}\right)_{x+\Delta x}+\bar{q} \bar{A} \Delta x= \\
& \bar{A} \frac{\partial(\bar{\Phi} \bar{\rho})}{\partial t} \Delta x
\end{aligned}
$$

onde $\rho$ é a massa especifica ou densidade, $\vec{V}$ o campo de velocidade, $t$ é o tempo, $A$ é a área do corte transversal para o fluxo, $q$ é a razão de massa de injeção por unidade de volume do reservatório (se o valor de $q$ for negativo implica produção). Se, por exemplo, a geometria do reservatório for dada por uma caixa de comprimento $x$ e o escoamento do fluxo por uma única fase, se pode dizer que a face esquerda (entrada) tem área $A(x)$, a face direita (saída) tem área $A(x+\Delta x$ ), (Peaceman, 1977).

A equação de continuidade pode ser escrita na forma simplificada como:

$$
\nabla \rho \vec{V}+\frac{\partial \rho}{\partial t}=0
$$

Onde $\nabla=\hat{i} \frac{\partial}{\partial x}+\hat{j} \frac{\partial}{\partial y}+\hat{k} \frac{\partial}{\partial z}$ é o operador vetorial gradiente.

Dois casos de escoamento para os quais a equação diferencial da continuidade pode ser simplificada (Fox e Mc Donald, 2001). Um primeiro caso diz respeito a escoamentos incompressíveis, isto é, escoamento onde a densidade não depende nem das coordenadas espaciais nem do tempo. Desta forma, a equação de continuidade é simplificada para

$$
\nabla \bullet \vec{V}=0
$$


Um segundo caso refere-se ao escoamento permanente, onde todas as propriedades dos fluidos, por definição, independem do tempo. Então, a equação de continuidade pode ser simplificada e apresentada na seguinte forma,

$$
\nabla \bullet \rho \vec{V}=0
$$

\subsection{2.}

\section{Equação da Quantidade de Movimento}

A equação de quantidade de movimento é responsável por descrever o movimento de um fluido. É a representação da segunda lei de Newton ou princípio fundamental da dinâmica, ou seja,

$$
\rho \frac{D \vec{V}}{D t}=\sum \text { Forças }
$$

Quanto maior for à força aplicada ao fluido maior será a aceleração adquirida pelo fluido (Fox e Mc Donald, 2001).

\subsection{3. \\ Equação de Energia}

Em um fluido, a energia total $i$ é considerada como sendo a soma da energia interna e da energia cinética por unidade de massa, conforme apresentado na equação (2.7).

$$
i=e+\frac{|\vec{V}|^{2}}{2}
$$

A equação de conservação de energia ou primeira lei da termodinâmica garante que as fontes para variação de energia total são o trabalho das forças agindo sobre o sistema e o calor total para este sistema, (Batchelor, 1967). 


\section{2.}

Propriedade da Rocha

Nesta seção se defina de uma forma muito breve dois conceitos fundamentais utilizados em modelagem de fluxo de meios porosos heterogêneos.

\subsection{1.}

Porosidade e compressibilidade

A porosidade de um meio é definida como a fração de volume total que é ocupada por um espaço vazio. O valor da porosidade está entre zero e um. O espaço vazio geralmente consiste em duas partes: o espaço poroso interconectado que está disponível para o fluxo de fluido e poros desconectados (becos sem saída) que não estão disponíveis para fluir. Usualmente a porosidade depende da pressão do reservatório (Peaceman, 1977). Então a compressibilidade da rocha pode ser definida como:

$$
c_{r}=\frac{1}{\Phi} \frac{d \Phi}{d P}=\frac{d \ln (\Phi)}{d P}
$$

Na equação (2.8), $c_{r}$ denota a compressibilidade da rocha, $\Phi$ representa à porosidade do meio e $\mathrm{P}$ a pressão do reservatório. Mas, por simplicidade do modelo proposto nesta pesquisa, a porosidade dependerá apenas das coordenadas espaciais. Portanto, para uma rocha com compressibilidade constante, a porosidade pode ser integrada por meio da equação (2.8) (Hasle et al, 2007), logo

$$
\Phi(P)=\Phi_{0} e^{c_{r}\left(P-P_{0}\right)}
$$

Para modelos simplificados é comum usar a linearização:

$$
\Phi=\Phi_{0}\left(1+c_{r}\left(P-P_{0}\right)\right)
$$

Outra importante propriedade da rocha é a permeabilidade absoluta que será definida a seguir. 


\subsection{2.}

Permeabilidade Absoluta

A permeabilidade absoluta $\vec{K}$, é uma característica intrínseca da rocha que mede a sua capacidade de transmitir fluidos em certas condições. Geralmente a permeabilidade varia de forma diferente em cada direção, mas muitas vezes são correlacionadas. Um meio poroso é chamado de isotrópico se a permeabilidade absoluta $\vec{K}$ for independente da direção, caso contrário é chamado de anisotrópico (Peaceman, 1977). Um caso isotrópico é considerado nesta pesquisa.

\section{3.}

\section{Lei de Darcy}

A lei de Darcy afirma que a densidade volumétrica do fluxo $V$ é proporcional ao gradiente de pressão do fluxo do fluido e um efeito devido à gravidade, (Darcy, 1856), ou seja:

$$
V_{\alpha}=-\vec{K} \frac{k r_{\alpha}}{\mu_{\alpha}}\left(\nabla P_{\alpha}-\left(-\rho_{\alpha} \vec{g} \nabla \text { Depth }\right)\right)
$$

Na equação acima, $\vec{K}$ é definido como a permeabilidade absoluta do meio poroso, $k r_{\alpha}$ a permeabilidade relativa da fase $\alpha, \mu_{\alpha}$ a viscosidade da fase $\alpha$ (a viscosidade é a resistência do fluido em escoar) e $\vec{g}$ a força gravitacional. O sinal negativo que está no inicio da equação (2.11) significa que o fluido escoa sempre de uma região com maior pressão para uma região com pressão mais baixa. A razão $\frac{\vec{K}}{\mu}$ pode ser entendida como o grau de condutividade do fluido no meio poroso.

\section{4.} Leis de Modelo de Brooks e Corey e Van Gebutchen

As permeabilidades relativas geralmente são assumidas para serem funções da saturação das fases conhecidas. Essas funções são normalmente obtidas a partir 
de experimentos físicos. A permeabilidade relativa $k r_{\alpha}$ descreve como uma fase $\alpha$ flui na presença de outra (Hasle et al, 2007).

A descontinuidade na pressão do fluido ocorre através de uma interface entre dois fluidos imiscíveis, no caso do presente trabalho, entre o fluido água e o óleo. Essa descontinuidade é chamada de pressão capilar definida pela diferença de pressão entre o fluido não molhante (óleo) e o fluido molhante (água), que é uma consequência da tensão superficial que existe na interface entre os dois fluídos. Na hidrodinâmica, os efeitos de capilaridade são responsáveis pelo surgimento de termos difusivos nas equações de transporte de fluidos, levando a uma suavização das frentes de onda (Chavent e Jaffré, 1986).

Outra propriedade importante é a mobilidade das fases que mede a facilidade com que os fluidos filtram com mais rapidez num meio poroso e é a razão entre a permeabilidade relativa $k r_{\alpha}$ e a viscosidade $\mu_{\alpha}$ da fase $\alpha$.

As curvas de Brooks e Corey (1964) são funções de permeabilidade relativa muito utilizadas para modelos bifásicos água e óleo (Chen et al, 2006). Portanto, essa pesquisa se baseou no modelo de Brooks-Corey para determinar a relação das permeabilidades relativas da água e do óleo e também da saturação dos mesmos. Então, segundo o modelo Brooks-Corey, se pode dizer que as permeabilidades relativas da água e do óleo são dadas por:

$$
\begin{aligned}
& k r_{w}\left(U_{w}\right)=\frac{\left(U_{w}-U_{r w}\right)^{2}}{\left(1-U_{r w}\right)^{2}} \\
& k r_{o}\left(U_{w}\right)=\left(1-\frac{U_{w}}{\left(1-U_{r o}\right)}\right)^{2}
\end{aligned}
$$

Na equação (2.12), $U_{r w}$ e $U_{r o}$ representam a quantidade mínima da água e do óleo presente no meio poroso, limitando os valores de saturação da água $U_{w}$ ao intervalo $\left[U_{r w}, 1-U_{r w}\right]$. A Figura 13 exibe as curvas de coeficientes de permeabilidades relativas da água e do óleo. Observa-se que, conforme a saturação da água cresce, a resistência à percolação (filtrar com mais rapidez) do óleo aumenta no meio poroso, fazendo com que a permeabilidade relativa do óleo $k r_{o}\left(U_{w}\right)$ diminua. 


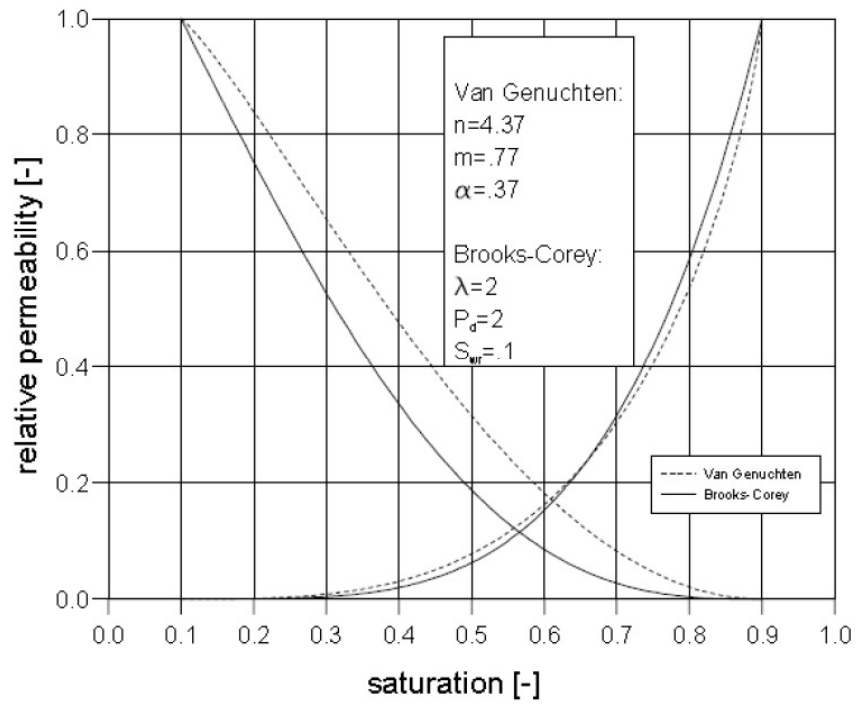

Figura 13: Relação entre a saturação e a permeabilidade relativa usando os modelos de BrooksCorey e Van Genuchten, Solidificação. (Marshall, 2009)

Considerando a hipótese que $U_{r w}=U_{r o}=0$ e $U=U_{w}$, as equações (2.12) pode ser reescrita de seguinte forma:

$$
\begin{aligned}
& k r_{w}\left(U_{w}\right)=U^{2} \\
& k r_{o}\left(U_{w}\right)=\left(1-U^{2}\right)
\end{aligned}
$$

Logo a mobilidade total é dada por:

$$
M_{T}=\frac{U^{2}}{\mu_{w}}+\frac{\left(1-U^{2}\right)}{\mu_{o}}
$$

E o fluxo fracional que será usado no algoritmo meshfree dada por:

$$
f(U)=\frac{U^{2}}{U^{2}+\mu(1-U)^{2}}
$$

onde $\mu$ a relação de viscosidade entre os dois fluidos, água e óleo, ou seja, $\mu=\frac{\mu_{w}}{\mu_{o}}$. A seguir será apresentada a derivada material. 


\section{5 .}

Derivada Material

No movimento de um corpo, a derivada material ou substantiva de um campo escalar, vetorial ou tensorial é a derivada total que mede a taxa de variação, no tempo, de uma grandeza associada a uma determinada partícula (Bird et al, 2007).

Dada uma função $f(x, t)$ qualquer, $x \in \Omega$, onde $\Omega$ é o domínio da função, a derivada da função, usando a regra da cadeia é:

$$
d f=\frac{\partial f}{\partial t} d t+\frac{\partial f}{\partial x} d x
$$

Dividindo ambos os membros por $d t$ tem-se:

$$
\frac{d f}{d t}=\frac{\partial f}{\partial t}+\frac{\partial f}{\partial x} \frac{d x}{d t}
$$

Assim a taxa de variação da função $f$ no tempo para um observador num ponto $x$ fixo, será dada por:

$$
\left.\frac{d f}{d t}\right|_{x}=\frac{\partial f}{\partial t}
$$

enquanto que a taxa de variação para um observador movendo-se com a partícula é dada por:

$$
\left.\frac{d f}{d t}\right|_{x}=\frac{\partial f}{\partial t}+\left.\frac{\partial f}{\partial x} \frac{d x}{d t}\right|_{x}
$$

A equação (2.19) pode ser reescrita da seguinte forma:

$$
\frac{D f}{D t}=\frac{\partial f}{\partial t}+\vec{V} \bullet \nabla f
$$

Onde $\vec{V}=\left.(d x / d t)\right|_{x}$ é a velocidade da partícula. A equação (2.20) é conhecida como a Derivada Material, Substantiva, Total ou Operador de Stokes, (Bird et al, 2007). 


\section{6.}

Advecção

Fluidos são meios materiais que, conforme se movimentam, transportam consigo propriedades mecânicas (massa, momento, etc.) e termodinâmicas (como a temperatura, por exemplo). Portanto, o movimento dos fluidos na advecção é descrito matematicamente como um campo vetorial, e o material transportado é tipicamente descrito como uma concentração escalar da substância, que está contida no fluido. Chama-se advecção justamente a propriedade de transporte de alguma característica do fluido (Deller, 2006).

A equação de advecção para uma quantidade conservada por um campo escalar $\phi$ é expressa matematicamente pela equação de continuidade, (Deller, 2006).

$$
\frac{\partial \phi}{\partial t}+\nabla \bullet(\phi \vec{V})=0
$$

Onde $\vec{V}=\left(V_{x}, V_{y}, V_{z}\right)$ é o campo de velocidade e $\nabla$ o operador divergente. Para o caso de incompressibilidade, isto é,

$$
\nabla \bullet \vec{V}=0
$$

ou seja, velocidade constante, conforme equação (2.4). Então a equação (2.22) pode ser reescrita na forma:

$$
\frac{\partial \phi}{\partial t}+\bar{V} \bullet \nabla \phi=0
$$

Que é idêntica ao segundo membro da equação de derivada material (2.20), sendo $f=\phi$.

\section{7.}

\section{Abordagens Lagrangeana e Euleriana}

$\mathrm{Na}$ abordagem Lagrangeana, o fluido é tratado como um sistema de partículas, pois cada nó ou ponto no fluido é classificado como tal. Cada partícula representa uma molécula ou um conjunto de moléculas do fluido e possui 
propriedades como posição, pressão, velocidade, saturação, entre outras. Enquanto que, na abordagem Euleriana, observam-se pontos fixos no espaço e analisam-se como as grandezas do fluido (tais como densidade, velocidade, temperatura) comportam-se naquele ponto fixo específico com o decorrer do tempo.

Em outras palavras, a diferença básica entre os esquemas Euleriano e Lagrangeano está no posicionamento do observador do escoamento (referencial). No Euleriano, o referencial é inercial, ou seja, é um ponto fixo no espaço onde uma determinada grandeza do escoamento é função do vetor posição em $\mathrm{R}^{3} \mathrm{e}$ do tempo $t$. Neste caso, a cada instante de tempo $t$ tem-se uma distribuição espacial de uma determinada grandeza (velocidade, temperatura, pressão e outros). No esquema Lagrangeano, utiliza-se um referencial não inercial, isto é, descreve-se a evolução do escoamento observado, acompanhando o movimento de uma parcela individual do fluido.

$\mathrm{Na}$ solução Euleriana para equação de advecção (2.23), observa-se a evolução do fluido mantendo uma distribuição regular dos seus pontos da grade, como pode ser visto na Figura 14. No tempo e num tempo $t+\Delta t$ são analisados os mesmos pontos da grade.

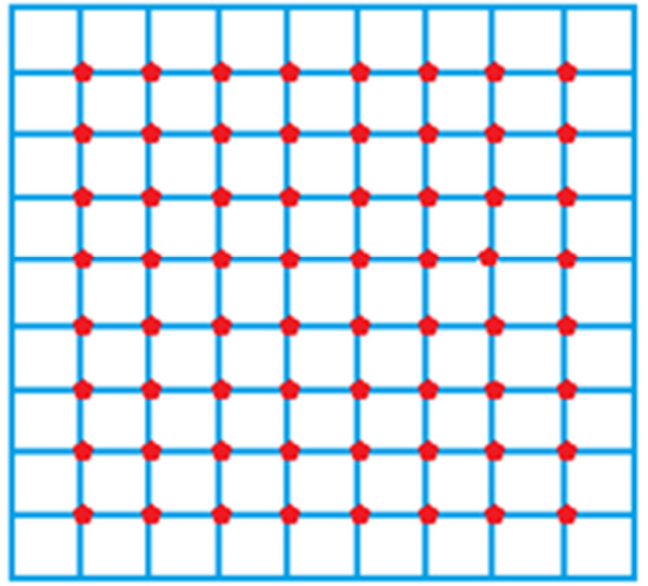

Euleriano no tempo $t$

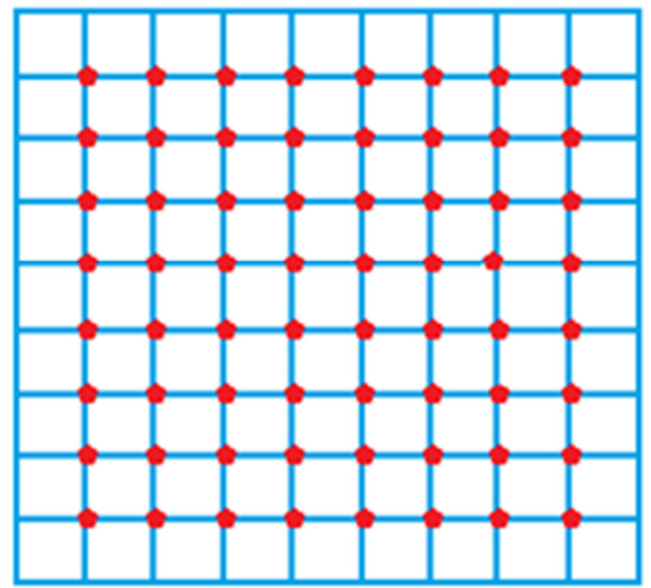

Euleriano no tempo $t+\Delta t$

Figura 14: Abordagem Euleriano 
Na solução Lagrangeana, a equação (2.19) passa a ser representada na forma Derivada Material (2.20), não explicitando-se, assim, o termo não linear (Mesinger e Arakawa, 1976) (Durran, 1998), de forma que o observador acompanha a partícula de fluido ao longo de sua trajetória no escoamento (Figura 15). Numericamente, a visão de Lagrange corresponde a um sistema de partículas, podendo existir ou não uma malha conectando estas partículas.

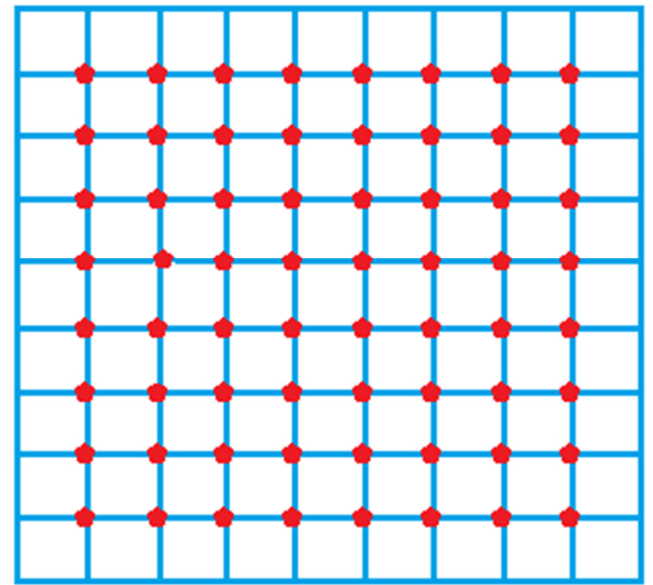

Lagrangeano no tempo $t$

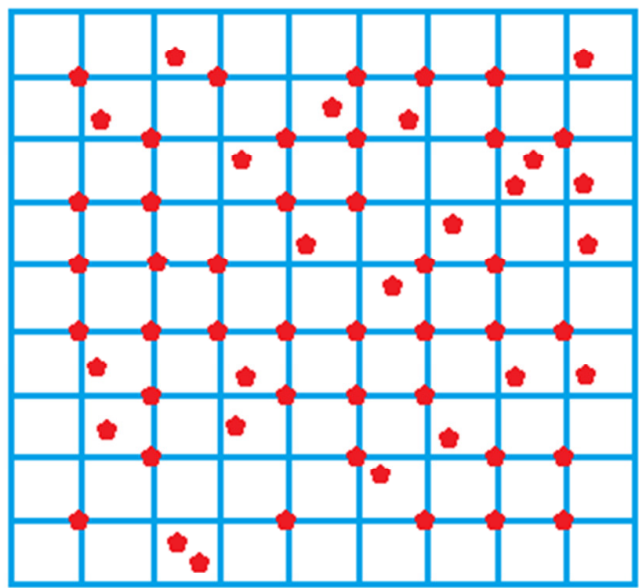

Lagrangeano no tempo $t+\Delta t$

Figura 15: Abordagem Lagrangeano

Uma das grandes desvantagens do esquema Lagrangeano é que um conjunto inicial de partículas regularmente distribuídas geralmente irá evoluir para uma distribuição espacial muito irregular (Welander, 1995), podendo, consequentemente, deixar de representar importantes características do escoamento (Staniforth e Cotê, 1991), (Figura 15). Na seção seguinte será apresentado o conceito do método Level Set, que faz uso tanto de uma abordagem Euleriana como de uma abordagem Lagrangeana.

\section{8.}

Conceito e Formulação do Método Level Set

O método Level Set é um método dinâmico de superfícies implícitas, isto é, simula e analisa movimentos de curvas, superfícies ou volumes (Osher e Fedkiw, 2003) (Sethian, 1999). A ideia é representar uma determinada curva (ou superfície 
ou volume) $\Gamma$ como uma curva de nível zero de uma superfície $\phi$ de maior dimensão, denominada função Level Set (Figura 16).

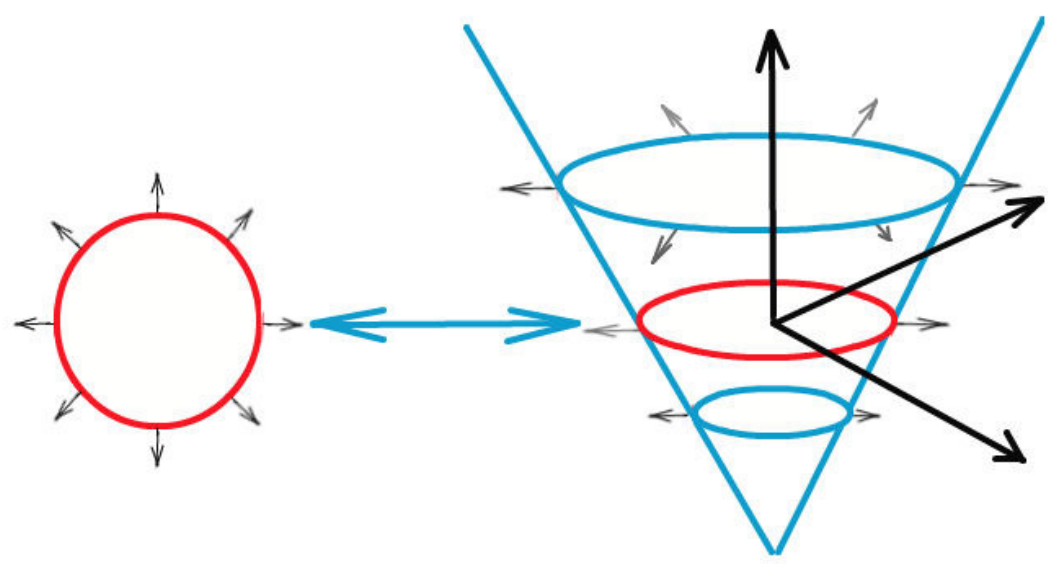

Figura 16: Level Set

A parte à esquerda da Figura 16 ilustra a expansão de uma circunferência e à direita da figura mostra a mesma circunferência como sendo a curva de nível zero de uma função de maior dimensão (Napolitano, 2004).

Uma das principais vantagens em utilizar o método Level Set se deve ao fato de que tal modelo numérico é capaz de tratar eficazmente mudanças topológicas e/ou descontinuidades que, eventualmente, podem surgir no decorrer da propagação da curva, superfícies ou volume de nível zero. Devido à eficiência do método Level Set, esta formulação tem sido amplamente aplicada em diversas áreas científicas, tais como, mecânica dos fluidos, processamento de imagens e visão computacional, crescimento de cristais, geometria computacional, ciência dos materiais, entre outras (Enright et al, 2002; Guedelman et al, 2005; Irving et al, 2006; Losasso et al, 2006; Sethian, 1999).

Após um breve relato introdutório do método Level Set, apresentam-se em síntese os fundamentos nos quais esse método está baseado.

Dada uma curva (ou superfície ou volume) $\Gamma$ como curva de nível zero (ou zero Level Set) qualquer, definida em certo domínio, assume-se que essa curva poderá se mover em uma direção normal a ela mesma de acordo com função 
velocidade $\vec{V}$. E que essa função pode assumir tanto valores positivos como, também, valores negativos.

A finalidade é escrever a equação de propagação dessa função chamada $\phi(\vec{x}, \vec{y}, \vec{z}, t)$, conhecida como função Level Set, que no caso unidirecional reduz-se a $\phi(\vec{x}, t)$ (Osher e Fedkiw, 2003). Considera-se a hipótese de que a posição de uma partícula descrita por $x(t)$, no caso unidirecional, esteja sobre a curva de nível $\phi(\vec{x}, t)$ (Napolitano, 2004), ou seja:

$$
\phi(\vec{x}(t), t)=0
$$

Derivando a função acima (equação 2.24) usando a regra da cadeia, tem-se

$$
\phi_{t}+\nabla \phi(\vec{x}(t), t) \cdot \dot{\vec{x}}(t)=0
$$

Sabendo que $\dot{\vec{x}}(t)$ na direção normal equivale a $\dot{\vec{x}}(t) \cdot \vec{N}$, esta corresponde à velocidade da partícula e é representada por $\vec{V}$, onde a componente normal $\vec{N}$ é dada por $\vec{N}=\nabla \phi /|\nabla \phi|$. Assume-se também que $\vec{V}(\vec{x})$ é conhecido para todos os pontos $x \operatorname{com} \phi(\vec{x})=0$. Como $\dot{\vec{x}}(t)=\vec{V}(x)$, então a equação acima (equação 2.25) pode ser reescrita da seguinte forma,

$$
\phi_{t}+\vec{V} \cdot \nabla \phi=0
$$

Onde o subscrito $t$ denota a derivada temporal parcial em relação à variável tempo $t, \vec{V}$ é a velocidade do campo para todos os pontos da superfície, representada por $\vec{V}=(u, v, w)$. E $\nabla$ o operador gradiente, dado por $\nabla=\frac{\partial}{\partial \vec{x}}+\frac{\partial}{\partial \vec{y}}+\frac{\partial}{\partial \vec{z}}$, então

$$
\vec{V} . \nabla \phi=u \phi_{x}+v \phi_{y}+w \phi_{z}
$$

Por fim pode-se reescrever a equação (equação 2.27) como,

$$
\phi_{t}+u \phi_{x}+v \phi_{y}+w \phi_{z}=0
$$

Onde $\phi_{x}, \phi_{y}$ e $\phi_{z}$, denotem as derivadas parciais em relação às variáveis espaciais $x, y$ e $z$ (Osher e Fedkiw, 2003). 
A equação (2.28) representa a equação diferencial parcial e define o movimento de uma interface, onde $\phi(\vec{x})=0$ (Napolitano, 2004), (Osher e Sethian, 1988).

O movimento do campo das velocidades no espaço bidimensional possui apenas a componente Normal $\vec{V}_{\vec{n}}$, isto é, a componente Tangencial $\vec{V}_{t}$ é zero (Osher e Fedkiw, 2003). Como dito anteriormente que a componente tangencial é nula, então a equação (2.26) é equivalente a

$$
\phi_{t}+V_{n} \vec{N} \cdot \nabla \phi=0
$$

Além disso, o produto escalar entre

$$
\vec{N} . \nabla \phi=\frac{\nabla \phi}{|\nabla \phi|} \nabla \phi=\frac{|\nabla \phi|^{2}}{|\nabla \phi|}=|\nabla \phi| .
$$

Logo, a equação de Level Set passa a ser escrita como,

$$
\phi_{t}+V_{n} \cdot|\nabla \phi|=0
$$

Onde $V_{n}$ é a componente da velocidade na direção normal.

Uma das leis mais importantes na simulação de fluidos é a lei de conservação de massa (seção 2.1.1). A grande desvantagem do método Level Set é que ele tem uma tendência de perda de massa de alta curvatura, isto é, o método Level Set não obedece à lei de conservação de massa (Osher e Fedkiw, 2003). Sendo assim, esta pesquisa preferiu representar o método Level Set numericamente através do esquema Semi-Lagrangeano que conserva massa e que será discutido no próximo capítulo. 


\section{3 Formulação do método Semi-Lagrangeano de advecção}

Este trabalho também faz uso de um método para acompanhamento de interfaces móveis, que se fundamenta no método de curvas de nível, Level Set, descrito no capitulo 2. O método será utilizado para problemas de advecção como transporte de um campo escalar. As interfaces são representadas através do método Semi-Lagrangeano, para simulação e visualização de dois fluidos, i.e. fluxo bifásico.

O esquema Semi-Lagrangeano é um método numérico que vem sendo amplamente utilizado em modelos de previsão numérica de tempo para integração das equações que governam o movimento atmosférico, conforme apresentado por Kalnay em 2003 em sua pesquisa. Recentemente, o mesmo método passou a ser aplicado a problemas de visualização e simulação na área de dinâmica dos fluidos computacionais (Iske e Käser, 2005).

Este método é chamado de Semi-Lagrangeano porque, conforme mencionado anteriormente, os métodos originais Lagrangeanos para simulação de fluidos são baseados em partículas, e não em malhas de posições fixas. Neste método, a "partícula" é apenas uma forma de refletir a resolução do sistema Euleriano, não aparecendo explicitamente no modelo numérico (Bridson et al, 2006).

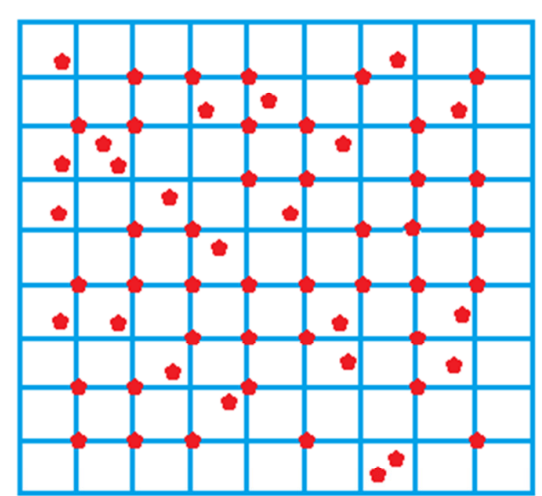

Semi-Lagrangeano no tempo $t$

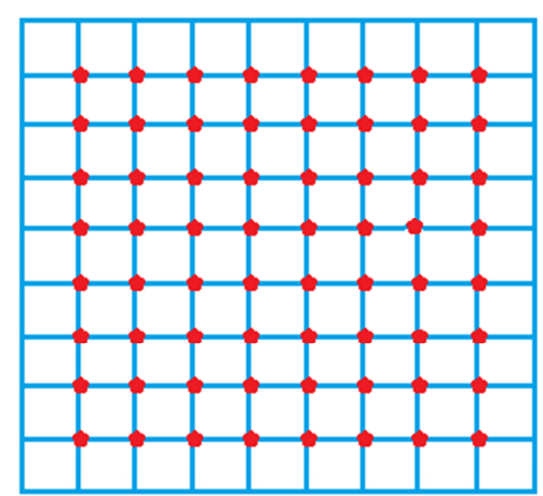

Semi-Lagrangeano no tempo $t+\Delta t$

Figura 17: Abordagem Semi-Lagrangeano 
A ideia principal do esquema Semi-Lagrangeano é conciliar as vantagens dos métodos Euleriano e Lagrangeano, isto é, a distribuição regular das partículas no tempo $t+\Delta t$ é mantida, conforme mostra a Figura 17. Deste modo, ao analisar o movimento de uma partícula imaginária transportando uma propriedade genérica $\phi$ ao longo de uma trajetória, pode-se utilizar o vetor velocidade para, a partir de um ponto $x_{t}$, retroceder a advecção até ao ponto de origem $x_{t_{0}}$, chamado de upstream point; e, neste ponto, verificar qual o valor que foi transportado do ponto $x_{t_{0}}$ para o ponto $x_{t}$ (Figura 18).

O ponto de origem é obtido de seguinte forma:

$$
x_{t_{0}}=x_{t}-\Delta t v_{x_{t}}
$$

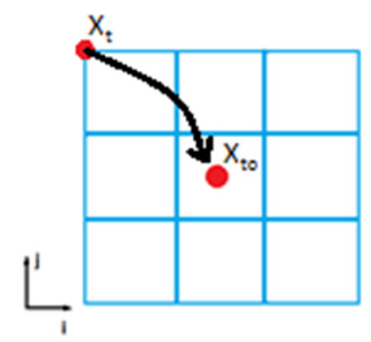

Figura 18: Upstream Point

Deve-se observar que, ao realizar a advecção a partir deste ponto, chega-se à partícula que está sendo analisada no momento. Isto é, o novo valor da propriedade $\phi$ em um ponto no espaço $x_{t}$ é justamente o antigo valor de $\phi$ que a partícula tinha ao partir do ponto $x_{t_{0}}$ e, desta forma, sabe-se de onde a partícula partiu, faltando apenas descobrir qual o seu valor de $\phi$ naquele ponto. Como normalmente o ponto inicial não é um ponto que foi amostrado na grade, utiliza-se uma interpolação para obter uma aproximação de seu valor. Esta interpolação pode ser bilinear ou trilinear ou usando interpolação por funções de base radial. Explicitando o valor de $\phi_{x_{t}}^{n+1}$ :

$$
\phi_{x_{t}}{ }^{n+1}=\operatorname{Interp} .\left(\phi_{n}, x_{t}-\Delta t v_{x_{t}}\right)
$$


Neste caso, para resolver o problema da interpolação local para achar a propriedade $\phi$ do upstream point $x_{t_{0}}$, esta pesquisa preferiu utilizar a interpolação por splines poliharmônicas - que é uma ferramenta para interpolação multivariada a partir de dados dispersos. A forma de interpolação multivariada será discutida mais a frente na seção (3.3).

Muitas propriedades em processo de transporte no tempo são descritos pelas leis de conservação hiperbólicas, suas equações que regem o movimento de uma ou de um conjunto de partículas, no caso linear, estão na forma:

$$
\frac{\partial \phi}{\partial t}+V \bullet \nabla f(\phi)=0
$$

Onde no domínio $\Omega \subset \Re^{d}$, com $d \geq 1$ e no intervalo de tempo $I=[0, T p]$, e $T p>0$, todos conhecidos, e a função $\phi: I \times \Omega \rightarrow \Re$ é desconhecida. Além disso, $f(\phi)=\left(f_{1}(\phi), \ldots, f_{d}(\phi)\right)^{T}$ denota o fluxo. A equação (3.3) é denominada como a equação de Buckley-Leverett (Iske e Käser, 2005). A equação de Buckley-Leverett é uma equação hiperbólica dependente do tempo, cuja solução $\phi$ apresenta descontinuidades, mesmo que os dados iniciais sejam suavizados, pois a descontinuidade é devido ao choque entre duas interfaces. Essa descontinuidade é usada nesta pesquisa como sendo o conceito da pressão capilar que é discutida com mais detalhes no capítulo 5.

Um caso especial da equação (3.3) é quando $f(\phi)=\phi$, no qual se obtém a equação na forma:

$$
\frac{\partial \phi}{\partial t}+\vec{V} \bullet \nabla \phi=0
$$

A equação (3.4) é do mesmo tipo que a equação (2.20) da derivada material e também, da equação (2.23) de advecção. Ainda, a equação (3.4) é também conhecida como advecção passiva.

Para modelar o choque entre as interfaces, (Iske e Käser, 2005) propuseram utilizar a equação (3.3) na forma não linear. Logo, o segundo membro da equação (3.3) torna-se diferente de zero. Portanto, por exemplo, a equação (3.3) pode ser utilizada de seguinte modo: 


$$
\frac{\partial \phi}{\partial t}+\vec{V} \bullet \nabla f(\phi)=\varepsilon \cdot \Delta \phi
$$

sendo, $\mathcal{E}$ o coeficiente de difusão e $\Delta$ o operador Laplaciano em $\Re^{d}$. A equação (3.5) também é conhecida como a equação de Buckley-Leverett viscoso. Segundo os autores Iske e Käser (2005), a ideia da modificação da equação (3.5) se baseia na técnica de estabilização, modificando assim, a equação hiperbólica (3.3) para uma equação parabólica (3.5), que ao contrário da equação (3.3) tem uma suavização da solução $\phi$ para qualquer passo de tempo, mesmo para os dados iniciais. Além disso, o coeficiente de difusão deve ser suficientemente pequeno.

A representação do problema é feita usando a ideia da abordagem Lagrangeana, pois, reescrevendo a equação (3.5) e usando o conceito de derivada material tem-se:

$$
\frac{D \phi}{D t}=\varepsilon \cdot \Delta \phi
$$

onde $\frac{D \phi}{D t}=\frac{\partial \phi}{\partial t}+\vec{V} \bullet \nabla f(\phi)$ é a derivada material mencionada na equação (2.20). Logo, a discretização da equação (3.6) é dada por:

$$
\frac{\phi(t+\tau, \xi)-\phi(t, x)}{\tau}=\varepsilon \cdot \Delta \phi(t, x)
$$

$x \equiv x(\xi) \equiv x_{t_{0}}$ é o upstream point correspondente ao nó $\xi \in \Xi$. Sendo $\Xi$ o conjunto dos pontos do domínio e os $\xi$ são os pontos da malha fixa do domínio do problema. A equação (3.7) pode ser ainda reescrita como:

$$
\frac{\phi(t+\tau, \xi)-\phi(t, \tilde{x})}{\tau}=\varepsilon . \Delta \phi(t, \tilde{x})
$$

Deve-se notar que o local exato de $x$ é desconhecido. Para computar a aproximação, $\tilde{x} \equiv x$, utiliza-se a evolução discreta da partícula no tempo, dada pela equação (3.1). Após tendo computado o upstream point, a aproximação desejada de $\phi(t+\tau, \xi)$ é dada por:

$$
\phi(t+\tau, \xi)=\phi(t, \tilde{x})+\tau . \mathcal{E} \cdot \Delta \phi(t, \tilde{x}), \quad \xi \in \Xi
$$


Para computar $\phi(t, \tilde{x})$ e $\Delta \phi(t, \tilde{x})$ utiliza-se a interpolação local por splines poliharmônicas $s$, satisfazendo a seguinte equação:

$$
s(\chi)=\phi(t, \chi) \quad \chi \in \eta
$$

A aproximação da interpolação local $s$, é feita determinando um conjunto de pontos vizinhos $\eta \subset \Xi$, ao redor do upstream point. Substituindo a equação (3.10) na equação (3.9) obtêm-se:

$$
\phi(t+\tau, \xi)=s(\tilde{x})+\tau . \varepsilon . \Delta s(\tilde{x}), \quad \xi \in \Xi
$$

A seguir será apresentado o algoritmo e o fluxograma do modelo com malha.

\section{1.}

\section{Algoritmo e o Fluxograma do Modelo com Malha}

O processo para resolver o movimento de um ou mais fluidos faz uso das equações de diferencias parciais tais como, Navier Stokes e ou equações BuckleyLeverett dependendo do problema a ser tratado. A solução dessas equações não é um processo numericamente simples. Com o intuito de simular numericamente estes movimentos, surge a necessidade de criar técnicas robustas, eficientes e confiáveis para discretização, representação e a determinação das grandezas envolvidas. De acordo com esta necessidade, Iske e Käser (2005) propuseram um algoritmo para resolver numericamente o método Semi-Lagrangeano, dividindo assim o problema em três etapas, como se segue:

\section{Dados de entrada:}

Passo de tempo $\tau$;

O conjunto dos nós da malha, conjunto $\Xi$;

Os valores de $\phi(t, \xi), \xi \in \Xi$.

\section{Loop principal:}

Para cada node $\xi \in \Xi$ faça:

1. Calcular os upstream points usando a equação (3.1);

2. Determinar $s(\tilde{x}) \approx \phi(t, \tilde{x})$ e $\Delta s(\tilde{x})$ por interpolação local;

3. Calcular $\phi(t+\tau, \xi)=s(\tilde{x})+\tau \cdot \varepsilon \cdot \Delta s(\tilde{x})$. 


\section{Dados de saída}

Os valores de $\phi(t+\tau, \xi)$ para todos $\xi \in \Xi$, para o tempo $t+\tau$.

Como dito anteriormente, a etapa 1, do loop principal, será aproximada usando a equação (3.1) e representada por um método eficiente e estável de resolução de equação diferencial ordinária. Obtendo, assim, a posição de todos os upstream points. A etapa 2 consiste em utilizar o conceito de interpolação local; assim, após ter determinado todos os upstream points pela etapa 1, calcula-se a aproximação de $s(\tilde{x})$ e $\Delta s(\tilde{x})$, individualmente, para cada upstream point, satisfazendo assim a equação (3.10). Logo, para cada upstream point, determinase um conjunto de pontos vizinhos, $\eta \subset \Xi$, ao redor de cada upstream point. São determinados 16 pontos, denominados stencil points, como mostra a Figura 19.

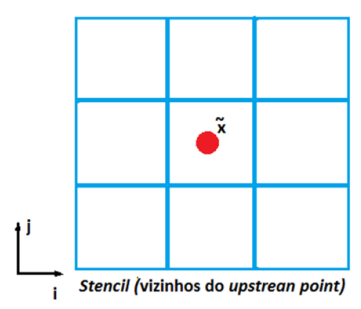

Figura 19: - Stencil Points.

Por fim, na etapa 3, determina-se a grandeza de interesse em todos os instantes de tempo, isto é, o valor do campo escalar $\phi(t+\tau, \xi)$, usando a equação (3.11). Lembrando que o valor do campo escalar $\phi(t+\tau, \xi)$, no tempo $t+\tau$ depende dos valores obtidos no instante anterior $t$ e assim sucessivamente.

$\mathrm{Na}$

Figura 20 é apresentado o fluxograma do método numérico Semi-Lagrangeano. 


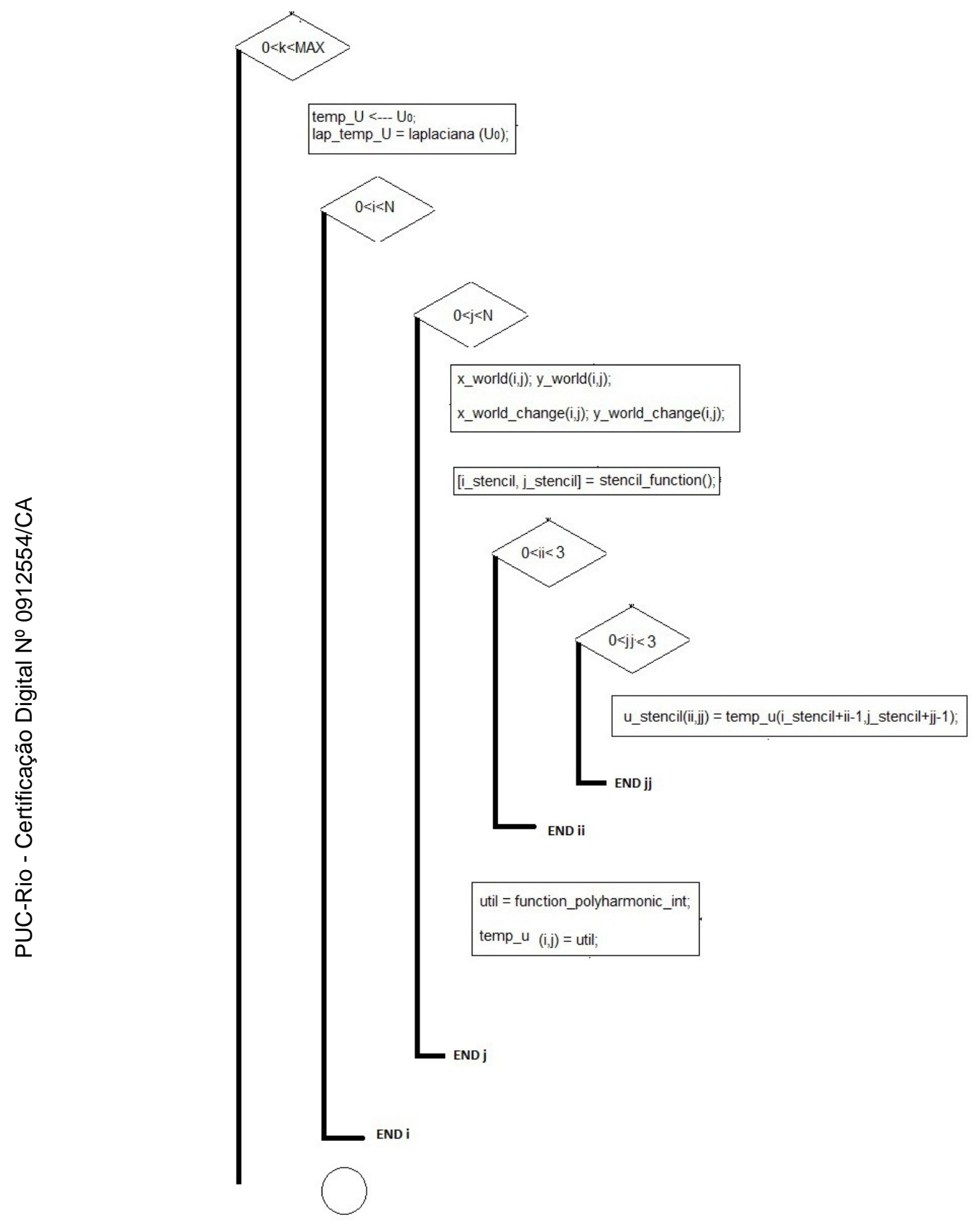




\section{Continuação}

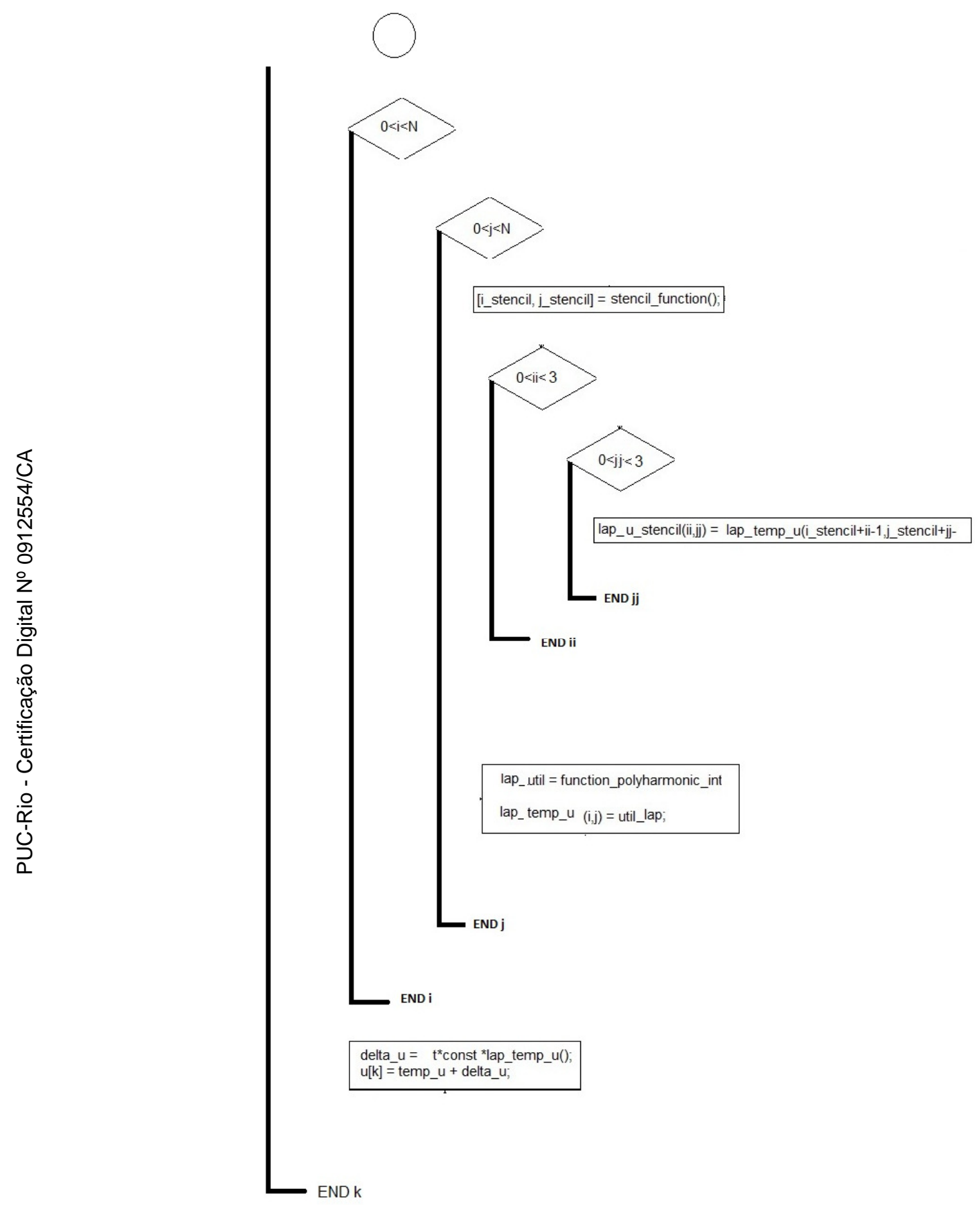

Figura 20: Fluxograma 


\section{2.}

\section{Algoritmo e o Fluxograma do Modelo Meshfree}

Até o momento discutiu-se a ideia de malha fixa, ou seja, o domínio era dado apenas por pontos igualmente espaçados. As informações, como pressão, velocidade e saturação eram determinadas para cada ponto da malha, como também a evolução do modelo em todos os instantes de tempo. Com o intuito de melhorar a eficiência no desempenho do modelo os autores Iske e Käser (2005) propuseram uma nova forma de determinar as informações da nova posição do upstream point, como também as informações de pressão, velocidade e saturação. Assim sendo, aqueles autores utilizaram a ideia de meshfree/Grid; portanto, neste caso, a nova posição da partícula é calculada por um conjunto de pontos gerados de forma aleatória (meshfree), enquanto que as informações de saturação são determinadas e atualizadas na malha fixa (Grid). Outra forma que os autores Behrens et al (2001) propuseram foi de utilizar apenas a ideia de meshfree (sem malha). Neste caso, a nova posição do upstream point, a pressão, a velocidade e a saturação são determinadas usando um conjunto de pontos gerados inicialmente de forma randômica. A variação do modelo no tempo é baseada no critério de avalição e estimação do erro gerado pela interpolação por splines poliharmônicas, que é apresentada na seção 3.3. Também, os autores Behrens et al (2001) utilizaram a ideia de refinamento e remoção de nós, que depende do erro gerado por cada partícula ou nó. Detalhes desta abordagem serão apresentados nas próximas seções.

Neste trabalho está sendo utilizada a ideia proposta pelos autores Behrens et al (2001), baseada em meshfree. Inicialmente um conjunto de pontos $\xi \in \Xi$ é gerado de forma randômica, onde $\Xi$ é o conjunto de todos os nós do domínio; e para cada ponto $\xi \in \Xi$ se determina, inicialmente, a pressão, velocidade e saturação.

A atualização da nova posição do upstream point é obtida através dos pontos gerados. A atualização da saturação é feita usando uma estratégia de avaliação e correção do erro $\eta(\xi)$. O erro $\eta(\xi)$ é obtido pela relação entre o valor da saturação do upstream point já interpolado, usando a função de interpolação por splines poliharmônicas e o valor da saturação advectado, dado pela equação (3.11). Determina-se o erro máximo dentre todos os erros $\eta(\xi)$. A adaptação do 
nó $\xi \in \Xi$, no esquema meshfree proposto pelos autores, é feita através da inserção (por inserção de pontos voronoi) e da remoção dos nós, dependendo do erro.

Os métodos meshfree podem ser computacionalmente caros, dependendo do número de pontos e, portanto, requerem regras eficazes e personalizadas para o refinamento e remoção de nós (Behrens et al, 2001). Por isso, é de extrema importância saber quando e onde as remoções e os refinamentos devem ser feitos.

A estratégia de adaptação do nó não contribui significativamente para o desempenho do método em termos de custos computacionais -contribui apenas na qualidade do modelo (Behrens et al, 2001).

A seguir é apresentado o algoritmo, discutindo detalhadamente cada passo.

\subsection{1. \\ Algoritmo}

Para cada nó $\xi$ do conjunto $\Xi \equiv \Xi^{t}$ (para o tempo $t$ ), uma aproximação do ponto $x$ correspondente ao upstream point é computado. As concentrações das saturações $U(t, x)$ são aproximadas pela interpolação por splines poliharmônicas, levando-se em conta os vizinhos do upstream point.

Para determinar todos os valores, $U(t+\tau, \xi)$, dados pela equação 3.11, cada nó $\xi \in \Xi$ pode ser simplificado ou removido, o que implicará na obtenção de um novo conjunto de nós, $\Xi \equiv \Xi^{t+\tau}$, para ser usado no próximo instante de tempo. A simplificação ou remoção do nó depende de regras propostas baseadas

no erro gerado $(\eta(\xi), \xi \in \Xi)$ pela interpolação em volta do nó $\xi$, como mencionado anteriormente.

A seguir é discutido o algoritmo do modelo de forma detalhada.

\section{Dados entrada}

Os valores que foram usados como dados de entrada para o problema de five spot são:

- Conjunto de pontos

- Número de passos de tempo;

- Discretização do tempo, deltaT;

- Posição dos poços; 
- Raio de injeção;

- Coeficiente de difusão;

\section{Condições iniciais e de contorno}

- Relação Pressão - Velocidade, neste caso foi dada pela lei de Darcy simplificada, isto é, a velocidade é igual a menos o gradiente de Pressão;

- Saturação inicial é dada pela relação entre a saturação da água e a saturação do óleo, ou seja, a soma dos dois é igual a 1.

\section{Dados de saída}

- Saturação $U(t+\tau, \xi)$ em cada instante de tempo. 


\section{Para cada instante de tempo faça: $t \rightarrow t+\tau$}

1. Iteraja até a Saturação do conjunto dos nós ficar estacionária.

Para cada ponto randômico $\xi \in \Xi$ faça:

2. Calcule a nova posição de $x \_u p s t r e a m$ equações (3.12) (3.14) e (3.15). Função ComputeUpstreamPointsBeta.

3. Determine os vizinhos de $x \_$upstream: função Neighbour.

4. Determine o valor da saturação $U$ do $x \_$upstream dada pela interpolação por splines poliharmônicas. Advecte $U$ já interpolado, onde,

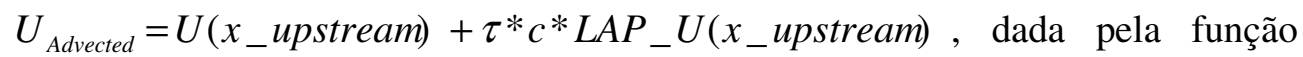
Semi_Lagrangian_Advect.

5. Determine o erro máximo entre o valor da saturação $U$ do $x \_$upstream e o valor da Saturação advectado, $\eta(\xi)=\mid U_{\text {Advected }}-U\left(x_{-}\right.$upstream $) \mid$. O erro máximo é dado por, ErroMax $=\max (\eta(\xi))$.

6. Refinamento/Simplificação, $\Xi \equiv \Xi^{t+\tau}$;

- Se o Erro de cada nó $\xi \in \Xi$ for menor do que $\theta_{\text {coarse }} *$ ErroMaxentão, remova $\mathrm{U}$.

- Se o Erro de cada nó $\xi \in \Xi$ for maior que $\theta_{\text {refine }} *$ ErroMax, refina, ou seja, insira novos pontos Voronoi e retorna ao passo 1 a 6 novamente.

- Senão a saturação $U=U_{\text {Advected }}$ obtida no passo 4 .

Função Adaptively_Refine_Coarse

Fim de cada ponto randômico $\xi \in \Xi$.

- Armazena todos os valores da saturação $U$ para cada instante de tempo, onde agora $\Xi \equiv \Xi^{t+\tau}$.

Fim do instante de tempo.

No algoritmo acima, são sempre armazenados dois conjuntos diferentes de nós, um no instante $\mathrm{t}$ (com valores conhecidos em locais conhecidos), e outro no instante $t \rightarrow t+\tau$, (valores e posições desconhecidos), que inicialmente são os mesmos, e que vai sendo gradativamente atualizado. 
No passo 2 calcula-se a trajetória da nova posição dos nós utilizando a aproximação do upstream point dada por

$$
x_{\text {Aprox }}=\xi-\beta
$$

Onde $\beta=\tau \cdot \vec{V}$, considerando a aproximação dependente do campo de velocidade.

A trajetória da partícula é acompanhada pela variação do campo de velocidade. Como mencionado anteriormente na seção 3.1, para um caso de advecção passiva (caso linear), a velocidade é dada por $\vec{V}=v$. Entretanto, para um caso não linear a velocidade de advecção é dada pela variação da função fluxo fracional, $f(U)$, com relação à saturação $U$, do passo anterior, dada por:

$$
\vec{V}=\frac{\partial f(U)}{\partial U}
$$

Neste caso específico, usando a equação de Buckley-Leverett, o campo de fluxo é dado pela equação (2.15) aqui reproduzida, onde os autores Iske e Käser (2005) propuseram utilizar $\mu$ igual a 0.5 .

É importante ter em mente que a função fluxo fracional $f(U)$, dependerá do tipo de equação a ser utilizada.

A aproximação de $\beta$, neste caso, foi obtida utilizando aproximação por fixed point iteration, baseado na ideia de midpoint, (Behrens et al (2002).

$$
\beta^{k+1}=\tau \cdot V\left(t+\frac{\tau}{2}, \xi-\frac{\beta^{k}}{2}\right)
$$

Note-se que a iteração acima é a evolução da velocidade $\vec{V}$ para um tempo intermediário, $t+\frac{\tau}{2}$. Então, como é um caso não linear, para computar $V\left(t+\frac{\tau}{2}, \xi-\frac{\beta^{k}}{2}\right)$, os autores, Behrens et al, (2002), propuseram empregar um esquema de extrapolação dado por:

$$
V\left(t+\frac{\tau}{2}, \xi-\frac{\beta^{k}}{2}\right)=\frac{3}{2} V\left(t, \xi-\frac{\beta^{k}}{2}\right)-\frac{1}{2} V\left(t-\tau, \xi-\frac{\beta^{k}}{2}\right)
$$


Assim, calculando-se o valor da saturação $U$ na posição $\xi-\beta$ e no tempo $t$, através da interpolação por splines poliharmônicas, é possível advectar a saturação $U$, para um tempo $t+\tau$, através da equação 3.11 .

No passo 3 do algoritmo, determinam-se os vizinhos do valor de upstream point aproximado, utilizando um critério de distância mínima entre os pontos gerados.

No passo 4, determina-se a valor da saturação $U$ do upstream point através da interpolação local usando splines poliharmônicas. Com os vizinhos do upstream point e os valores conhecidos da saturação dos vizinhos obtem-se o valor da saturação do upstream point requerido.

No passo 5, calcula-se o erro máximo local a partir do cálculo dos erros de todos os vizinhos. O erro é dado pela diferença do valor da saturação do upstream point advectado (equação 3.11) menos o valor da saturação $U$ calculado pela interpolação por splines poliharmônicas, isto é $\eta(\xi)=|U(\xi)-s(\xi)|$. Este erro indicará se deve haver remoção ou refinamento.

$\mathrm{O}$ erro é $\eta(\xi)$ devido principalmente à função base radial utilizada durante a interpolação. A escolha da função base é crucial para a avalição do erro do modelo, dependendo do problema. A função base utilizada na interpolação por splines poliharmônicas é conhecida como Thin Plate, (Wu e Schaback, 1993).

No passo 6, aplica-se um processo de refinamento ou remoção dos nós, com base no erro máximo calculado no passo 5. Caso o erro local esteja acima de certo limite, definido pelo usuário, refina-se localmente essa área. Caso o erro local esteja abaixo de um limiar, também definido pelo usuário, remove-se o nó.

São escolhidos os valores $\theta_{\text {refine }}$ e $\theta_{\text {coarse }}$, onde $0 \leq \theta_{\text {coarse }}<\theta_{\text {refine }} \leq 1$, para definir quando deve haver refinamento ou remoção:

- Se o erro $\eta(\xi)$ de um nó $\xi \in \Xi$ for menor do que $\theta_{\text {coarse }} *$ ErroMax, então remova $\xi$. 
- Se o erro $\eta(\xi)$ de um nó $\xi \in \Xi$ for maior que $\theta_{\text {refine }} *$ ErroMax, então refine, ou seja, insira novos pontos Voronoi.

- Caso contrário a saturação é dada por $\mathrm{U}=U_{\text {Advected }}$, isto é, a saturação é a mesma obtida no passo 4.

Valores sugerido pelos autores Behrens et al (2001) foram $\theta_{c r s}=0.1$ e $\theta_{r e f}$ $=0.2$, mas isso depende do critério a ser proposto pelo usuário, dependendo do problema a ser tratado.

Com isto tem-se um novo conjunto de nós com novas informações. De posse deste novo conjunto de nós o algoritmo retorna para o passo 2. Os passos 2 a 6 se repetem até não encontrar mais regiões, nem para refinar nem para remover, isto é, a repetição para quando a iteração converge. Para mais detalhes ver as seções (3.2.1 e 3.2.2).

Um conjunto de nós é considerado estacionário se menos que um percentual dos nós, definido pelo usuário, sofreu refinamento ou simplificação. Segundo os autores Behrens et al (2001), 1\% é um valor adequado. Essa abordagem evita loops de muitas iterações.

\section{3. Interpolação Spline Poliharmônica}

Splines poliharmônicas são usadas para aproximações de funções e interpolação de dados dispersos de várias dimensões, ou seja, multiescala. Nesta interpolação, os dados do vetor $\left.f\right|_{x}=\left(f\left(x_{1}\right), f\left(x_{2}\right) \ldots, f\left(x_{n}\right)\right)^{T} \in \mathfrak{R}^{N}$ são valores de uma função desconhecida $f: \Re^{d} \rightarrow \Re$ para um conjunto de pontos dispersos $X=\left\{x_{1}, x_{2} \ldots, x_{n}\right\} \subset \mathfrak{R}^{d}$ de $n$ variáveis independentes, assumindo $d \geq 1$ (Iske, 2003). A interpolação desses dados dispersos deve satisfazer a seguinte condição: $s: \Re^{d} \rightarrow \mathfrak{R}$, tal que $\left.s\right|_{x}=\left.f\right|_{x}$, isto é,

$$
s\left(x_{j}\right)=f\left(x_{j}\right), \text { para } 1 \leq j \leq N
$$


A interpolação poliharmônica é dada por:

$$
s(x)=\sum_{j=1}^{N_{P}} c_{j} \varphi_{d}\left(\left\|x-x_{j}\right\|\right)+v^{T}\left[\begin{array}{l}
1 \\
x
\end{array}\right]
$$

Onde $c_{j}$ são os $N_{P}$ pesos das funções base de acordo com o número de pontos do stencil, $x \in X$, $\|$ é a norma Euclidiana em $I R^{d}, v$ são os $n+1$ pesos do polinômio, obtidos a partir da equação (3.21) e $n$ é o grau do polinômio; neste caso $n=2$, pelo motivo do problema ser $2 D$. As funções $\varphi_{d}(r)$ são as funções de base radial, dadas por:

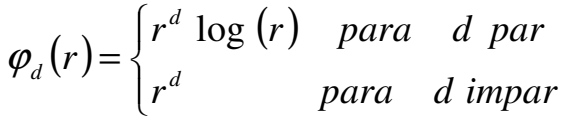

$$
\begin{aligned}
& r=\left\|x-x_{j}\right\|_{2}=\sqrt{\left(x-x_{j}\right)^{T}\left(x-x_{j}\right)}
\end{aligned}
$$

Os pesos $c_{j}$ e $v_{i}$ são determinados de tal modo que a função passa através dos $N_{p}$ pontos dados e devem satisfazer a condição de ortogonalidade:

$$
0=\sum_{j}^{N_{P}} c_{j}, \quad 0=\sum_{j}^{N_{P}} c_{j} x_{i, j} \quad i=1,2, \ldots n
$$

Para computar os pesos $c_{j}$ e $v_{i}$ resolve-se a seguinte sistema de equações lineares:

$$
\left[\begin{array}{ll}
A & B^{T} \\
B & 0
\end{array}\right]\left[\begin{array}{l}
c \\
v
\end{array}\right]=\left[\begin{array}{l}
Y \\
0
\end{array}\right]
$$

onde:

$$
A_{j, i}=\varphi\left(\left\|x_{j}-x_{i}\right\|\right), \quad B=\left[\begin{array}{ccccc}
1 & 1 & 1 & \ldots & 1 \\
x_{1} & x_{2} & \ldots & x_{N_{P}}
\end{array}\right],
$$

onde:

$$
Y=\left[y_{1}, y_{2}, \ldots, y_{N_{P}}\right]^{T}
$$


A matriz do sistema de equações lineares para determinar os pesos é não singular, portanto existe uma única solução.

A seguir serão apresentadas algumas vantagens e desvantagens do método SemiLagrangeano.

\section{4 .}

Vantagens e desvantagens do Método Semi-Lagrangeano

Devido à necessidade de calcular-se sempre a posição de partida da partícula e das interpolações locais, a implementação da abordagem SemiLagrangeana implica num maior custo computacional por passo de tempo. Entretanto, para melhorar a eficiência do método, é possível utilizar passos de tempo grandes, uma vez que, matematicamente, o esquema é estável. A interpolação sempre será obtida através de valores conhecidos da partícula em questão para obter o novo valor. Portanto, o domínio de dependência da solução numérica incluirá sempre o domínio de dependência da solução exata, (Durran, 1998).

Uma das limitações dos métodos numéricos explícitos é a restrição de estabilidade imposta pela condição Courant-Friedrich-Lewy (condição CFL) (Courant et al, 1928). A grande vantagem do método Semi-Lagrangeano é a capacidade de resolução de fenômenos transientes sem se preocupar muito com o passo de tempo, ou seja, passos de tempo arbitrários.

Apesar de estável para qualquer $\Delta t$, este método acarreta uma perda de volume. Isto significa que não pode ser utilizado para realizar a advecção de campos que necessitem de uma alta precisão. No entanto, o método SemiLagrangeano pode ser utilizado na simulação de substâncias difusivas como a fumaça, pois a perda de volume tende a ser natural, (Durran, 1998). 


\section{4 \\ Formulação dos métodos meshfree}

Uma malha é uma rede formada por ligação dos nós de forma pré-definida, podendo ser uma grade, volumes, células ou elementos. Ao contrário de um meshfree (sem malha) que é um conjunto de nós espalhados dentro do domínio do problema, para representar, e não discretizar, o domínio do problema e suas fronteiras. Não há nenhuma relação ou conectividade entre os nós espalhados pelo domínio, ou seja, não estão interconectados, são linearmente independentes.

Os métodos meshfree, também chamados de métodos de partículas, são métodos numéricos para resolução de equações diferencias parciais com condições de contornos ou iniciais que usa um critério de aproximação de forma local, ou global, para obter soluções de valores pontuais, (Gingold e Monaghan, 1977), (Wendlan, 2005), (Fasshauer, 2007). Os métodos meshfree são bastante utilizados em estudos de problemas que envolvam grandes deformações e propagação de fraturas, problemas com fronteiras e ainda processos adaptativos (Liu, 2003), (Belytschko et al, 1996), (Gu et al, 2014), (Aitken et al, 2015).

De forma mais detalhada, é possível caracterizar os métodos meshfree da seguinte forma:

- Representação do domínio - Um conjunto de nós de forma aleatória é escolhido para representar o domínio do problema e a sua fronteira. Vale a pena ressaltar que o maior problema dos métodos meshfree está na modelagem das fronteiras. Além disso, a quantidade de nós também é uma restrição no domínio problema. O domínio neste trabalho foi composto por um conjunto de Halton Points (Halton, 1960; Wong et al, 1997) e a condição de fronteira utilizada foi a condição de contorno de Dirichlet.

- Interpolação do campo - A interpolação é usada para aproximação dos nós. Esta pesquisa adotou interpolação por splines poliharmônicas como funções de base radial. 
- Formulação de sistemas de equações - As equações dos métodos meshfree podem ser formuladas usando sistemas de equações na forma forte ou fraca. Esta tese utilizou a solução forte na formulação.

- De acordo com cada problema, a resolução das equações diferenciais parciais globais pode ser feita através de métodos direto ou indireto, implícito ou explicito, entre outras formas de resolução levando em conta a precisão, estabilidade e velocidade. Esta pesquisa utilizou formas diretas de resolução das equações diferencias parciais. As aproximações foram feitas explicitamente de forma global ou local dependendo do caso.

Para finalidade deste trabalho, preferiu-se trabalhar com os métodos baseados nas aproximações de funções de base radial $(R B F)$ de Hermite simétrico e RBF Kansa antissimétrico, (Kansa, 1990b), (Fasshauer, 2007). Segundo Gregory Fasshauer (2007), esses métodos fornecem uma reconstrução numericamente estável de arbitrária ordem de aproximação local a partir de dados esparsos. A formulação desses métodos será detalhada nas próximas seções. Por agora pode-se dizer que os métodos de Kansa e Hermite são poderosos na teoria da aproximação multivariada de dados esparsos, especialmente, permitem trabalhar em arbitrária dimensão do espaço.

Nesta pesquisa foram implementados os métodos meshfree de aproximação por RBF Hermite simétrico e RBF Kansa antissimétrico de dados esparsos, principalmente, para o cálculo do Laplaciano de dados dispersos. Em seguida será descrito o processo geral e fundamental dos métodos de aproximação esparsa anteriormente mencionados.

\section{1.} Métodos Meshfree

Nesta seção serão introduzidas algumas técnicas de resolução de equações diferencias parciais $(E D P)$ por meio de funções de base radial $R B F$ para dados esparsos. As técnicas são baseadas em aproximações pelo método de Kansa e o método de Hermite, (Fasshauer, 2007), mencionados anteriormente. Esse conceito 
é visto na literatura como uma forma de solução forte quando se trata de simulações de propriedades físicas baseadas em aproximações meshfree. Além disso, consideram-se as condições de contorno de Dirichlet para ajudar no tratamento e na resolução das equações diferenciais parciais. A seguir será discutido o método de Kansa.

\subsection{1.}

Aproximação por método antissimétrico de Kansa

Considera-se um domínio composto por $\left\{x_{i}, f\right\}, i=1, \ldots, N, x_{i} \in R^{s}$, onde os pontos são esparsamente distribuídos em $R^{s}$, ou seja, um conjunto de pontos não interconectados. A função escalar $f$ representa a imagem do espaço dimensão, dada por uma função suave (Fasshauer, 2007). A imagem desses pontos pode ser aproximada por uma relação entre os pesos de cada ponto e uma função de suavização escolhida de acordo com o problema, isto é:

$$
\mathrm{P}_{f}=\sum_{j=1}^{N} \lambda_{j} \varphi\left(\left\|x-x_{j}\right\|\right), \quad x \in R^{s}
$$

$$
\Lambda=\left\{\lambda_{1}, \ldots \lambda_{N}\right\}
$$

Onde $\Lambda$ é o conjunto de pesos de cada ponto. De tal modo que:

$$
\mathrm{P}_{f}\left(x_{i}\right)=f_{i} \quad i=1 \ldots N
$$

Para determinar os pesos este problema leva à determinação de um sistema linear $(a x=b), A \lambda=f$, sendo que a matriz $A$ é dada por:

$$
A_{i, j}=\varphi\left(\left\|x_{i}-x_{j}\right\|\right), \quad i, j=1 \ldots, N
$$

A equação (4.4) denota imagem da função $\varphi$ da distância entre todos os pontos pertencentes ao domínio. Para uma melhor aproximação a matriz $A$ é dada localmente, isto é, pela distância de todos os pontos considerados na vizinhança do ponto onde se quer obter a propriedade física e também pela distância dos pontos colocados na fronteira dados pela condição de Dirichlet. 
Por motivos de possíveis singularidades, Kansa (1990b), afirma que a matriz $A$ deverá ser necessariamente não singular. Uma das formas de evitar as singularidades, neste caso é solucionar através de colocação de pontos para resolver as equações diferenciais parciais, utilizando condição de contorno de Dirichlet. Portanto, assumindo que o domínio $\Omega \subset R^{s}$ seja uma equação diferencial parcial elíptica na forma:

$$
\operatorname{Lu}(x)=f(x) \quad x \quad \text { em } \Omega
$$

Onde $L$ é o operador diferencial e também considerando a condição de fronteira de Dirichlet dada por:

$$
u(x)=g(x), \quad x \quad \text { em } \partial \Omega
$$

Onde $g(x)$ é uma função dada para os pontos da fronteira $\partial \Omega$. Pode-se dizer que baseado na aproximação dada na equação (4.1), Kansa prefere representar a solução aproximada de $\hat{u}$ por uma base radial de expansão, da seguinte forma:

$$
\hat{u}(x)=\sum_{j=1}^{N} \lambda_{j} \varphi\left(\left\|x-\xi_{i}\right\|\right)
$$

Note que o parâmetro $\hat{u}$ para esta pesquisa denota o campo escalar, isto é, o campo de saturação dos upstream points. Onde $\Xi=\left\{\xi_{1} \ldots, \xi_{N}\right\}$ são os centers ou o conjunto de pontos dados na vizinhança do upstream point se for considerada uma aproximação local, ou o conjuntos de todos os pontos do domínio se for considerada uma aproximação global. $\mathrm{O}$ conjunto $X=\left\{x_{1} \ldots x_{N}\right\} \subset \Omega$ representa o ponto ou o conjunto de pontos da aproximação pretendida. Embora formalmente diferentes em algumas situações, estes pontos fisicamente coincidem. Nesta pesquisa o conjunto de pontos $X$ representam os upstream points. A solução da equação (4.7) pode ser considerada para uma análise local ou global para solucionar a propriedade física escalar no ponto requerido, de acordo com o problema. Combinando a ideia das equações (4.5 e 4.6), surge a matriz de colocação antissimétrica, dada por:

$$
A=\left[\begin{array}{l}
\tilde{A}_{L} \\
\tilde{A}
\end{array}\right]
$$


A matriz de colocação $\widetilde{A}_{L}$ é dada pela imagem do operador diferencial da função de suavização da distância entre os pontos originais, (centers, $\Xi$ ), de acordo com a equação (4.5). Enquanto que a matriz de colocação $\tilde{A}$ é a imagem da função de suavização da distância dos pontos colocados na fronteira de acordo também com condição de Dirichlet dada na equação (4.6). Como se pode notar nas equações (4.9) e (4.10).

$$
\begin{gathered}
\left(\tilde{A}_{L}\right)_{i, j}=L \varphi\left(\left\|x-\xi_{j}\right\|\right)_{x=x_{i}}, \quad x_{i} \in \mathrm{I}, \quad \xi_{j} \in \Xi \\
\tilde{A}_{i, j}=\varphi\left(\left\|x_{i}-\xi_{j}\right\|\right), \quad x_{i} \in \mathrm{B}, \quad \xi_{j} \in \Xi
\end{gathered}
$$

Em outras palavras, $\tilde{A}$ é a imagem do conjunto dos pontos colocados na fronteira $B$ para o tratamento de borda. Observe que o problema somente será bem colocado se, e somente se, o sistema linear $A \lambda=f$, resultar em uma solução única. A função $\varphi$ é a função base radial utilizada. No método original, Kansa propôs utilizar a função base multiquadratics dada por,

$$
\varphi=\frac{1}{\sqrt{1+r^{2}}}
$$

Mas nesta pesquisa preferiu-se trabalhar com a função base radial por splines poliharmônicas para uma melhor aproximação apresentada na seção (3.3). O método de Kansa pode ser bem adequado para vários outros problemas que envolvem solução de equações diferenciais parciais e dependentes do tempo.

A seguir será analisado o método simétrico de Hermite. É importante observar que a ideia de aproximação de Hermite se baseia nos mesmos conceitos do método de aproximação de Kansa, sendo a grande diferença na colocação da matriz A para solucionar os pesos de todos os pontos. 


\subsection{2.}

\section{Aproximação pelo método simétrico de Hermite}

O método de Hermite é baseado na abordagem de interpolação generalizada de Hermite, interpolação de Lagrange, Teorema de Taylor multivariada e colocação simétrica para equações diferenciais parciais. O método de Kansa bem como o método de Hermite é eficiente na solução de problemas de equações diferencias parciais $P D E$ com condições de contorno, neste caso, condição de fronteira de Dirichlet. Como mencionado anteriormente, o problema pode ser estendido para problemas lineares e não lineares de dados esparsos. A ideia do método de Hermite de colocação da matriz Hermitiana assente principalmente no tratamento das singularidades que possivelmente podem aparecer no problema durante a simulação. Assim sendo, Hermite propõe que a matriz $A$ também seja não singular. Seguindo a mesma ideia de Kansa, utilizando as equações (4.5) e (4.6), Hermite propõe a seguinte expansão para uma função desconhecida (Fasshauer, 2007):

$$
\begin{aligned}
& \hat{u}(x)=\sum_{j=1}^{N_{I}} \lambda_{j} L^{\xi} \varphi(\|x-\xi\|)_{\xi=\xi_{i}}+ \\
& \sum_{j=N_{I}+1}^{N} \lambda_{j} \varphi\left(\left\|x-\xi_{j}\right\|\right)
\end{aligned}
$$

Onde $N_{I}$ é o número de nós no interior do domínio e $L^{\xi}$ é o operador diferencial para ser usado na aproximação da equação. O operador $L \varphi$, da aproximação Kansa, bem como o operador $L^{\xi} \varphi$ são diferenciais da função de suavização $\varphi$ da distância entre os pontos interiores (de 1 a $N_{I}$ ) e dos pontos colocados na fronteira (de $N_{I+1}$ a $N$ ). Ambos serão utilizados para o cálculo da matriz Hermitiana que determina os pesos de todos os pontos para serem usados na equação (4.11). Portanto, isso pode ser de forma local considerando apenas a vizinhança como também de forma global considerando todos os pontos do domínio.

Observe que a colocação das equações (4.5) e (4.6) é agora da seguinte forma:

$$
\begin{aligned}
& L u\left(x_{i}\right)=f\left(x_{i}\right) \quad x_{i} \in I \\
& u\left(x_{i}\right)=g\left(x_{i}\right), \quad x_{i} \in B
\end{aligned}
$$


Note que o conjunto $I$ é dado por todos os pontos no interior do domínio e que o conjunto $B$ é dado por todos os pontos colocados na fronteira. Então, diferentemente da matriz $A$ de Kansa dada na equação (4.8), agora a colocação de Hermite é dada como:

$$
A=\left[\begin{array}{ll}
\tilde{A}_{L L}{ }^{\xi} & \tilde{A}_{L} \\
\tilde{A}_{L}{ }^{\xi} & \tilde{A}
\end{array}\right]
$$

Em outras palavras e de forma mais detalhada a montagem da matriz Hermitiana $A$ é caracterizada por:

$$
\begin{gathered}
\left(\tilde{A}_{L L}^{\xi}\right)_{i, j}=L L^{\xi} \varphi\left(\left.\|x-\xi\|\right|_{x=x_{i}, \xi=\xi_{j}}, \quad x_{i}, \xi_{j} \in \mathrm{I},\right. \\
\left(\tilde{A}_{L}\right)_{i, j}=L \varphi(\|x-\xi\|)_{x=x_{i}}, \quad x_{i} \in \mathrm{I}, \quad \xi_{j} \in B, \\
\left(\tilde{A}_{L}^{\xi}\right)_{i, j}=L^{\xi} \varphi\left(\left\|x_{i}-\xi\right\|\right)_{\xi=\xi_{j}}, \quad x_{i} \in B, \quad \xi_{j} \in \mathrm{I}, \\
\tilde{A}_{i, j}=\varphi\left(\left\|x_{i}-\xi_{j}\right\|\right), \quad x_{i}, \xi_{j} \in \mathrm{B} .
\end{gathered}
$$

Observe também os operadores diferencias $L \varphi \mathrm{e} L^{\xi} \varphi$, para este problema, representam o laplaciano da função base radial da distância entre os pontos interiores e dos pontos colocados na fronteira. E que o operador $L L^{\xi}$, neste caso, matematicamente significa $\nabla^{2} \nabla^{\xi}$.

Note que a matriz de colocação de Hermite é um pouco mais complexa na estruturação e requer funções de base mais suaves do que a matriz de colocação de Kansa. A expansão do método de aproximação de Hermite usando a matriz simétrica é uma eficiente colocação para determinar a variação de propriedades físicas de pontos esparsos tanto para problemas lineares como para problemas não lineares. Para uma boa convergência usando alta dimensão em domínios totalmente irregulares, as funções de base radiais devem ser necessariamente mais suaves, segundo a análise feita por Franke e Schaback (1998). (Chaback, 1998b), (Wendland, 2005a).

No ano 2003, os autores Larsson e Fornberg, (2003), concluíram que o método de colocação simétrico de Hermite é mais preciso do que o método de 
colocação antissimétrico de Kansa. Computacionalmente falando, o método antissimétrico de Kansa é mais barato que o método simétrico de Hermite na montagem da matriz de colocação $A$ por requerer menos derivadas da função base radial, mais simples de programar e, além disso, pode ser aplicado à problemas com soluções menos suaves (Fasshauer, 2007).

Para mais informações sobre o tema pode-se referenciar alguns autores de teoria de aproximações, tais como, como, Buhmann (2003), Cheney e Light (1999), Fasshauer (2007), Iske (2004), Wendland (2005) e Firoozjaee et al (2015). Acrescentam-se também trabalhos que exploraram a teoria de aproximações com aplicações na engenharia, tais como: Forrester et al (2008), Liu (2009), Griebel e Schweitzer (2014).

A seguir será apresentada uma proposta para simulação de fluidos bifásicos, imiscíveis e incompressíveis num meio poroso heterogêneo considerando um domínio tridimensional. A ideia dessa proposta é combinar os métodos de colocação meshfree com um meio poroso heterogêneo. 


\section{5 Novo modelo de fluxo multifásico de fluidos imiscíveis e incompressíveis em meios porosos heterogêneos.}

Os modelos numéricos de reservatórios são usados para estimar a produção, calibrar parâmetros e visualizar o fluxo de um reservatório de petróleo. Portanto, o objetivo principal desses modelos é fornecer uma base de informações em comparação com dados experimentais para auxiliar na projeção da posição e no gerenciamento dos poços a fins de maximizar a recuperação de petróleo e gás (Barker e Thibeau, 1997; Christie e Blunt, 2001; Hasle et al, 2007).

Segundo Rosa et al (2006), os modelos de simulação de reservatórios podem ser classificados entre físicos (e.g. modelos reduzidos e protótipos) e matemáticos; sendo os modelos matemáticos, por sua vez, subdivididos entre analíticos (e.g. o modelo de Buckley-Leverett para fluidos não miscíveis) e numéricos (i.e. aqueles que usam soluções numéricas para estudar modelos de reservatórios mais complexos). Este último tipo de simulador é geralmente denominado de simuladores numéricos de fluxo. Além disso, os modelos numéricos de um reservatório de fluxo são caracterizados pelo número de fases admitidas (monofásico, bifásico e trifásico - e.g. óleo e água no caso bifásico), pelo número de dimensões do domínio $(1 D, 2 D$, ou $3 D)$ e pelas hipóteses e tratamento matemático usados com relação à representação dos comportamentos físicos e das características de desempenho. Uma hipótese importante diz respeito às condições do meio poroso, que pode ser homogêneo ou heterogêneo. Geralmente, os reservatórios de fluxo são heterogêneos.

A Figura 21 ilustra um fluxograma simplificado para o desenvolvimento de um simulador numérico de reservatório. 


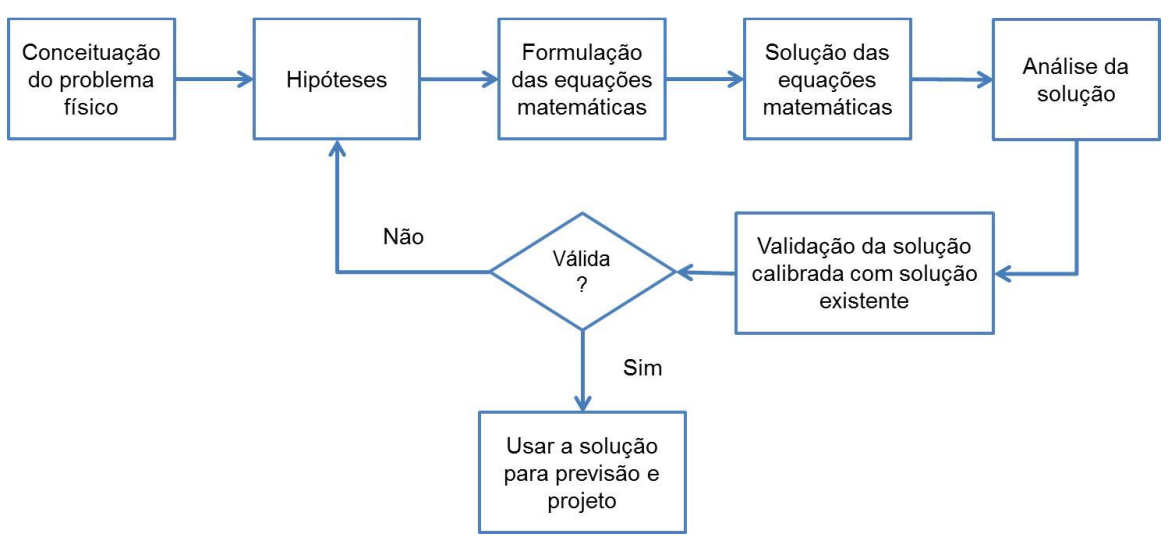

Figura 21: Fluxograma de um simples modelo numérico de reservatórios (Adalberto et al, 2006).

O modelo físico usado no desenvolvimento de um simulador numérico de reservatório é o escoamento de fluidos em meios porosos. Diversas são as hipóteses e condições que podem ser consideradas neste modelo, tais como homogeneidade ou heterogeneidade, geometria, condições de contorno apropriadas, anisotropia ou isotropia, a miscibilidade ou imiscibilidade, compressibilidade ou incompressibilidade, consideração ou não dos efeitos de capilaridade, efeitos gravitacionais ou ainda as forças viscosas. Todas desempenhando um papel importante no processo de fluxo de fluido. $\mathrm{Na}$ resolução de problemas físicos a escolha dessas hipóteses pode ser crucial no resultado do modelo requerido (Peaceman, 1977; Christie e Blunt, 2001; Hasle et $a l$, 2007). Durante a simulação em um reservatório de petróleo, também é importante impor condições de injeção e produção, como por exemplo, impor que o termo fonte seja nulo, em todo o domínio, exceto nos poços de injeção.

Essas hipóteses são discutidas com mais detalhes neste capítulo. Por enquanto, pode-se dizer que a geometria desempenha um papel importante na visualização dos modelos, como também, a condição de contorno imposta dá suporte à simulação do reservatório. Nesta pesquisa utilizou-se a condição de fronteira de Dirichlet apresentado na equação (4.6).

Um dos modelos mais utilizados para descrever o fluxo de fluidos em meios porosos em um reservatório é o modelo "Black Oil". Este modelo usa uma descrição simples de Pressão-Volume-Temperatura $(P V T)$ na qual as várias espécies químicas de hidrocarbono são agrupadas em um único componente simples, isto é: o óleo é considerado um único componente de hidrocarbono pesado (embora seja constituído por diversos hidrocarnonetos), assim como o gás 
é representado por um único componente de hidrocarbono leve. Neste caso, o componente simples gás pode ser parcial ou completamente dissolvido na fase óleo, formando uma ou duas fases (líquido ou vapor) que não se dissolve na fase água. Quando não se tem esta simplificação de agrupar componentes, passa-se a lidar com modelos composicionais. Há também os modelos térmicos, quando é necessário considerar os efeitos de variações da temperatura no interior do reservatório (e.g. injeção de vapor ou água quente em poços). Os modelos térmicos são necessariamente composicionais. Há vários outros tipos de modelos, tais como IMPES, generalizado e porosidade simples/dual. Alguns destes modelos se referem à aplicação (e.g. o térmico), outros à formação do modelo em si (e.g. IMPES) e outros se referem a um atributo da formação do reservatório (e.g. porosidade simples/dual). Uma apresentação clara destes tipos de modelo pode ser encontrada na publicação online da SPE denominada PetroWiki (http://petrowiki.org/Reservoir_simulation).

Os grupos mais conhecidos na sociedade de engenharia de petróleo por abordar o modelo "Black Oil" são o Computer Modeling Group Ltd (CMG, 2007) e o grupo SINTEF (Aarnes et al, 2007). Os modelos bifásicos são muito utilizados na indústria de reservatórios. Um dos métodos mais utilizados para abordar os modelos bifásicos é a metodologia IMPES (Implicit Pressure - Explicit Saturation) (Chen et al, 2004), que é usada no desacoplamento das equações governantes com intuito de solucioná-las separadamente. O modelo IMPES também pode ser estendido para situações trifásicas, quando passa a ser denominado de formulação sequencial.

É importante ressaltar que, na abordagem IMPES, o campo de pressão é determinado de forma implícita e a saturação de forma explícita. O campo de saturação é atualizado a cada instante de tempo utilizando os valores mais recentes do campo de pressão; portanto, o campo de pressão evolui mais lentamente do que o campo de saturação. Geralmente os métodos explícitos são limitados por uma condição de estabilidade durante o avanço temporal (Chen et al, 2004).

O petróleo é formado principalmente por compostos de carbono e hidrogênio, denominados de hidrocarbonetos. Inicialmente, no processo de produção de petróleo, um reservatório de hidrocarboneto está em equilíbrio contendo gás, óleo e água, separados pela gravidade (Peaceman, 1977; Hasle et al, 
2007). Quando um poço é perfurado, este equilíbrio é perturbado e o fluxo "explode" porque o reservatório está sob grande pressão. Segundo Hasle et al (2007), durante este processo de produção, aproximadamente vinte por cento dos hidrocarbonetos presentes são produzidos até que um novo equilíbrio é alcançado. Este processo é denominado de produção primária. Durante o processo de produção primaria haverá uma queda de pressão e menos óleo e gás fluem e a produção passa a não ser mais economicamente sustentável. Em geral, para compensar esta queda, utiliza-se a recuperação secundária, que consiste na injeção da água com objetivo de deslocar o óleo na direção dos poços de produção.

A presente pesquisa foca na simulação e visualização de recuperação secundária. Ainda é possível obter produção através da recuperação terciária, onde se promove a injeção de solventes que reduzem a viscosidade do petróleo, facilitando o seu movimento no interior do reservatório; aquecendo, assim, o reservatório por injeção de substâncias sofisticadas, tais como espuma, polímeros ou solventes (Hasle et al, 2007). Os polímeros devem mudar as propriedades de fluxo da água e, desse modo, empurrar o óleo de forma mais eficiente para os poços de produção (Peaceman, 1977).

A finalidade principal da presente pesquisa é a simulação e a visualização de um fluxo bifásico num meio poroso heterogêneo, considerando os efeitos de capilaridade entre os fluidos água e óleo e os efeitos de gravidade, utilizando os conceitos de meshfree. Naturalmente, uma desvantagem dos métodos de meshfree em aplicações de grande escala na indústria é o aumento do tempo de processamento computacional na resolução das matrizes dos sistemas lineares ou não lineares, principalmente na evolução temporal desses modelos. Uma das formas de reduzir o tempo de processamento dos modelos de grande escala consiste em desenvolver algoritmos paralelos baseados nas unidades de processamento gráfico conhecido como GPUs (Graphics Processing Units). No desenvolvimento deste trabalho foram utilizadas funções em GPU para acelerar a solução dos sistemas lineares do tipo $A x=b$, implementadas em MATLAB (Martin, 2010).

O Modelo proposto é um modelo de duas fases (água e óleo), incompressível, imiscível em meios porosos heterogêneos usando uma geometria tridimensional, considerando os efeitos das seguintes forças: capilares, viscosas e gravitacionais. No primeiro momento, a geometria regular é usada nas três 
direções, desprezando os efeitos gravitacionais. No segundo momento, é empregado o conceito de meshfree que se baseia, principalmente, como mencionado no capítulo 4, nas aproximações do método de Kansa e do método de Hermite para aproximar todas as componentes variacionais como gradiente e divergente na resolução das equações diferencias parciais. A análise feita também despreza os efeitos de gravidade.

A formulação matemática do modelo proposto descreve as equações de correlação pressão-velocidade e a saturação da água, usando a lei de Darcy e as leis de conservação de massa para escoamentos imiscíveis em meios porosos heterogêneos. A escolha das estratégias para resolução do problema matemático é extremamente importante bem como os métodos numéricos a serem utilizados. Nesta pesquisa, utilizou-se a abordagem do método característico modificado para advectar o campo de saturação num meio poroso heterogêneo. Mais detalhes são mencionados ao longo deste capítulo.

A seguir é apresentada a formulação matemática do modelo incompressível e imiscível, de duas fases em meios porosos heterogêneos, utilizando um domínio tridimensional.

\section{1. Nova formulação do modelo de fluxo bifásico de fluidos incompressíveis e imiscíveis em meios porosos heterogêneos tridimensionais.}

Nesta seção é discutida a formulação do modelo de um fluxo bifásico de fluidos incompressíveis e imiscíveis em meios porosos heterogêneos tridimensionais. Este modelo se baseia na consideração de todas as forças e efeitos mencionados anteriormente, isto é, efeitos viscosos, efeitos de capilaridade e efeitos gravitacionais. Além disso o modelo está preparado para lidar com reservatórios homogêneas ou heterogêneas, com geometria regular ou meshfree. As equações diferenciais são obtidas através da combinação da lei de Darcy e a equação da continuidade para cada fase. Nos instantes iniciais se discute a equação diferencial parcial de pressão-velocidade e, em seguida, apresenta-se a equação diferencial parcial da saturação, descrevendo assim o fluxo para as duas fases (água e óleo) em geometria tridimensional. Alguns conceitos básicos da 
mecânica de reservatório descritos no capítulo 2 são utilizados, também, nesta seção.

O espaço vazio no meio poroso é suposto ser preenchido com as diferentes fases $\alpha$, onde $\alpha=o, w, g$; isto é, $\alpha$ é óleo (o), água ( $w$ ) ou gás ( $g$ ). A fração de volume de cada fase é a saturação $\mathrm{U}_{\alpha}$ de cada fase $\alpha$.

Cada fase contém um ou mais componentes $l$. Na hipótese do Black oil, os vários componentes são agrupados em um único componente e, portanto, pode-se considerar um único componente para hidrocarbonos representando o óleo, outro componente para gás e outro para a água. A fração de massa $\gamma$ do componente $l$ na fase $\alpha$ é denotada por $\gamma_{1 \alpha}$. Em cada uma das fases, as frações de massa devem somar 1 e, portanto, para $N$ componentes diferentes, tem-se que:

$$
\sum_{l=1}^{N} \gamma_{l o}=\sum_{l=1}^{N} \gamma_{l w}=\sum_{l=1}^{N} \gamma_{\mathrm{lg}}=1
$$

O modelo que descreve o fluxo de um fluido de vários componentes e várias fases é a equação de conservação para cada componente $l$ (Peaceman, 1977; Hasle et al, 2007):

$$
\begin{aligned}
& \frac{\partial}{\partial t}\left(\Phi \sum_{\alpha} \gamma_{l \alpha} \rho_{\alpha} U_{\alpha}\right)+\nabla \bullet\left(\sum_{\alpha} \gamma_{l \alpha} \rho_{\alpha} \vec{V}_{\alpha}\right) \\
& =\sum_{\alpha} \gamma_{l \alpha} q_{\alpha}
\end{aligned}
$$

$$
\alpha=o, w, g
$$

onde $\Phi$ representa a porosidade da rocha, $\gamma_{l \alpha}$ a fração volumétrica (fração de massa) do componente $l$ na fase $\alpha, \vec{V}_{\alpha}$ a velocidade da fase $\alpha$ e $q_{\alpha}$ é a fonte da fase $\alpha$. Considerando um fluxo imiscível de apenas duas fases (uma fase de água e uma fase de óleo) a equação (5.2) se reduz a duas equações, (Hasle et al, 2007):

$$
\begin{gathered}
\frac{\partial}{\partial t}\left(\Phi \rho_{\alpha} U_{\alpha}\right)+\nabla \bullet\left(\rho_{\alpha} \vec{V}_{\alpha}\right)=q_{\alpha} \\
\alpha=w, o
\end{gathered}
$$


Dependendo do modelo a ser simulado é de extrema importância à colocação de hipóteses para simplificar ou generalizar a complexidade do modelo.

\section{Hipóteses}

1. Para simplificação considera-se a ausência da troca de massa entre as fases água e óleo.

2. As densidades não variam nem em relação ao tempo nem em relação ao espaço.

3. Fluidos são incompressíveis, isto é, $\nabla \bullet \vec{V}=0$ (equação 2.4), a densidades das fases óleo e água $\left(\rho_{o}\right.$ e $\left.\rho_{w}\right)$ são constantes.

4. A porosidade $\Phi$ depende apenas das coordenadas espaciais.

5. Meio poroso isotrópico, ou seja, a permeabilidade absoluta é a mesma na direção e sentido.

6. Estruturas são heterogêneas, pois há uma variação da porosidade e a permeabilidade.

7. $U=U_{w}+U_{o}=1, U=U_{w}$, segundo o modelo de Corey, (1956) (Brooks e Corey, 1964).

Aplicando a regra da cadeia e dividindo ambos os membros pela densidade $\rho_{\alpha}$ na equação (5.3), logo se obtém:

$$
\begin{aligned}
& \frac{1}{\rho_{\alpha}} \frac{\partial}{\partial t}(\Phi) \rho_{\alpha} U_{\alpha}+\frac{1}{\rho_{\alpha}} \Phi \rho_{\alpha} \frac{\partial}{\partial t}\left(U_{\alpha}\right)+ \\
& \Phi \frac{U_{\alpha}}{\rho_{\alpha}} \frac{\partial}{\partial t}\left(\rho_{\alpha}\right)+\frac{1}{\rho_{\alpha}} \rho_{\alpha} \nabla \bullet\left(\vec{V}_{\alpha}\right)+\frac{\vec{V}_{\alpha} \nabla \rho_{\alpha}}{\rho_{\alpha}}=\frac{q_{\alpha}}{\rho_{\alpha}}
\end{aligned}
$$

Note que: 


$$
\begin{aligned}
& \frac{\partial}{\partial t}\left(\Phi \rho_{\alpha} U_{\alpha}\right)=\frac{\partial}{\partial t}(\Phi) \rho_{\alpha} U_{\alpha}+\Phi \rho_{\alpha} \frac{\partial}{\partial t}\left(U_{\alpha}\right)+ \\
& \Phi U_{\alpha} \frac{\partial}{\partial t}\left(\rho_{\alpha}\right) \\
& \nabla \bullet\left(\rho_{\alpha} \vec{V}\right)=\rho_{\alpha} \nabla \bullet\left(\vec{V}_{\alpha}\right)+\vec{V}_{\alpha} \nabla \rho_{\alpha}
\end{aligned}
$$

Simplificando a equação (5.4) acima e escrevendo para ambas as fases, se tem:

$$
\begin{aligned}
& \frac{\partial}{\partial t}(\Phi) U_{w}+\Phi \frac{\partial}{\partial t}\left(U_{w}\right)+\Phi \frac{U_{w}}{\rho_{w}} \frac{\partial}{\partial t}\left(\rho_{w}\right)+ \\
& \nabla \bullet\left(\vec{V}_{w}\right)+\frac{\vec{V}_{w} \nabla \rho_{w}}{\rho_{w}}=\frac{q_{w}}{\rho_{w}} \\
& \frac{\partial}{\partial t}(\Phi) U_{o}+\Phi \frac{\partial}{\partial t}\left(U_{o}\right)+\Phi \frac{U_{o}}{\rho_{o}} \frac{\partial}{\partial t}\left(\rho_{o}\right)+ \\
& \nabla \bullet\left(\vec{V}_{o}\right)+\frac{\vec{V}_{o} \nabla \rho_{o}}{\rho_{o}}=\frac{q_{o}}{\rho_{o}}
\end{aligned}
$$

Somando ambas às equações de continuidade de ambas as fases e de acordo com as hipóteses (4) e (7), a equação (5.6) pode ser reescrita de seguinte forma:

$$
\begin{aligned}
& \frac{\partial}{\partial t}(\Phi)+\Phi \frac{U_{w}}{\rho_{w}} \frac{\partial}{\partial t}\left(\rho_{w}\right)+\Phi \frac{U_{o}}{\rho_{o}} \frac{\partial}{\partial t}\left(\rho_{o}\right) \\
& +\nabla \bullet\left(\vec{V}_{w}+\vec{V}_{o}\right)+\frac{\vec{V}_{w} \nabla \rho_{w}}{\rho_{w}}+\frac{\vec{V}_{o} \nabla \rho_{o}}{\rho_{o}} \\
& =Q
\end{aligned}
$$

Também sé possível perceber que:

$$
\begin{aligned}
& \frac{\partial}{\partial t}(\Phi) U_{w}+\frac{\partial}{\partial t}(\Phi) U_{o}= \\
& \frac{\partial}{\partial t}(\Phi)\left(U_{w}+U_{o}\right)=\frac{\partial}{\partial t}(\Phi) \\
& \frac{q_{w}}{\rho_{w}}+\frac{q_{o}}{\rho_{o}}=Q
\end{aligned}
$$


Para introduzir a pressão da água $P_{w}$ e a pressão do óleo $P_{o}$ na equação (5.7), usa-se a compressibilidade da rocha dada pela equação (2.8) e considera-se que a compressibilidade da fase é definida como no caso da rocha, isto é:

$$
\begin{gathered}
c_{r}=\frac{1}{\Phi} \frac{\partial \Phi}{\partial P} \\
c_{w}=\frac{1}{\rho_{w}} \frac{\partial \rho_{w}}{\partial P} \\
c_{o}=\frac{1}{\rho_{o}} \frac{\partial \rho_{o}}{\partial P_{o}}
\end{gathered}
$$

Onde $P$ é a pressão do reservatório. No momento oportuno, é importante lembrar que, no caso simplificado da rocha e das fases fluidas serem incompressíveis, se tem que $c_{r}=c_{w}=c_{o}=0$ e, como consequência de se desprezar a compressibilidade da rocha, assume-se que a porosidade da rocha $\Phi$ depende apenas das coordenadas espaciais. Considerando que o campo de velocidade é dado pela lei de Darcy, também apresentada na equação (2.11) do capítulo 2, temse que substituindo as equações (5.9) na equação (5.7), chega-se a:

$$
\begin{aligned}
& \nabla \bullet\left(\begin{array}{l}
-\vec{K} M_{w}\left(\nabla P_{w}-\rho_{w} \vec{G}\right)- \\
\vec{K} M_{o}\left(\nabla P_{o}-\rho_{o} \vec{G}\right)
\end{array}\right) \\
& +c_{r} \Phi(x) \frac{\partial P}{\partial t}+\Phi(x) \frac{U w_{w}}{\rho_{w}} c_{w} \rho_{w} \frac{\partial P_{w}}{\partial t}+ \\
& \Phi(x) \frac{U_{o}}{\rho_{o}} c_{o} \rho_{o} \frac{\partial P_{o}}{\partial t}+ \\
& \frac{\left(-\vec{K} M_{w}\left(\nabla P_{w}-\rho_{w} \vec{G}\right)\right) c_{w} \rho_{w} \nabla P_{w}}{\rho_{w}}+ \\
& \frac{\left(-\vec{K} M_{o}\left(\nabla P_{o}-\rho_{o} \vec{G}\right)\right) c_{o} \rho_{o} \nabla P_{o}}{\rho_{o}} \\
& =Q \quad
\end{aligned}
$$


Ainda no capítulo 2 pôde ser visto que a mobilidade total é dada pela soma da mobilidade da água $M_{w}$ com a mobilidade do óleo $M_{o}$, ou seja $M_{T}=M_{w}+M_{o}$. Portanto, simplificando a equação (5.10) tem-se, então, a seguinte equação geral:

$$
\begin{aligned}
& -\nabla \bullet\left(\vec{K} M_{w}\left(\nabla P_{w}-\rho_{w} \vec{G}\right)+\vec{K} M_{o}\left(\nabla P_{o}-\rho_{o} \vec{G}\right)\right) \\
& +c_{r} \Phi(x) \frac{\partial P}{\partial t}- \\
& c_{w}\left(\nabla P_{w} \bullet \vec{K} M_{w}\left(\nabla P_{w}-\rho_{w} \vec{G}\right)-\Phi(x) U_{w} \frac{\partial P_{w}}{\partial t}\right) \\
& -c_{o}\left(\nabla P_{o} \bullet \vec{K} M_{o}\left(\nabla P_{o}-\rho_{o} \vec{G}\right)-\Phi(x) U_{o} \frac{\partial P_{o}}{\partial t}\right) \\
& =Q
\end{aligned}
$$

Com se pode observar na equação geral (5.11) é possível determinar o campo de pressão da água, o campo de pressão do óleo, a pressão total, e as saturações da água e do óleo.

Geralmente todos os fluidos são compressíveis, mas usualmente a compressibilidade da água e do óleo são desprezíveis (Peaceman, 1977). Então pela hipótese (3), desprezando a compressibilidade da rocha, a compressibilidade da água e do óleo na equação (5.11), ou seja, $c_{r}=c_{o}=c_{w}=0$, tem-se:

$$
\begin{gathered}
\nabla \bullet\left(\begin{array}{l}
-\vec{K} M_{w}\left(\nabla P_{w}-\rho_{w} \vec{G}\right)- \\
\vec{K} M_{o}\left(\nabla P_{o}-\rho_{o} \vec{G}\right)
\end{array}\right)=Q \\
\nabla \bullet\left(\vec{V}_{w}+\vec{V}_{o}\right)=\nabla \bullet\left(\vec{V}_{T}\right)=Q
\end{gathered}
$$

Observa-se que na equação (5.12) ou (5.13) a velocidade de Darcy total é $\vec{V}_{T}=\vec{V}_{w}+\vec{V}_{o}$, isto é:

$$
V_{T}=-\vec{K} M_{T} \nabla P_{T}+\vec{K}\left(M_{w} \rho_{w}+M_{o} \rho_{o}\right) \vec{G}
$$


A equação (5.14) será utilizada para determinar o campo de pressão e o campo de velocidade em todo o domínio. Desta maneira, como o objetivo principal é obter o campo de saturação em cada instante de tempo e como a mobilidade total $M_{T}$ depende do campo de saturação, então a correlação de pressão-velocidade será dada atualizando-se automaticamente o campo de saturação. Além disso, a pressão da água $P_{w}$ e a pressão do óleo $P_{o}$ são desconhecidas na equação (5.12). Para isso, é usual eliminar uma das pressões, introduzindo na equação o conceito de pressão capilar. Por definição pressão capilar é uma diferença de pressão entre duas fases, ou seja, uma descontinuidade de pressão que ocorre através de uma interface entre as duas fases imiscíveis, neste caso, óleo e água. Logo,

$$
\begin{aligned}
& P_{c}=P_{o}-P_{w} \\
& P_{c}=P_{o}-P_{T} \\
& P_{T}=P_{o}-P_{c} \\
& \nabla P_{c}=\nabla P_{o}-\nabla P_{w}
\end{aligned}
$$

Sendo assim, aplicando novamente a definição da lei de Darcy para as duas fases e as relações de pressões dadas na equação (5.15) (Peaceman, 1977), tem-se a seguinte relação:

$$
\begin{aligned}
& \vec{K} M_{o} M_{w} \nabla P_{c}=M_{o} V_{w}-M_{w} V_{o}+ \\
& \vec{K} M_{o} M_{w}\left(\rho_{o}-\rho_{w}\right) \vec{G}
\end{aligned}
$$

A ideia é eliminar uma das velocidades. Substituindo, $V_{o}=V_{T}-V_{w}$, na equação (5.16) tem-se:

$$
\begin{aligned}
& \vec{K} M_{o} M_{w} \nabla P_{c}=M_{o} V_{w}-M_{w}\left(V_{T}-V_{w}\right)+ \\
& \vec{K} M_{o} M_{w}\left(\rho_{o}-\rho_{w}\right) \vec{G}
\end{aligned}
$$

Observa-se que o objetivo é obter o campo de velocidade da fase água, então, simplificando, obtém-se:

$$
V_{w}=\frac{M_{w}}{M_{o}+M_{w}}\left(\begin{array}{l}
\vec{K} M_{o} \nabla P_{c}+V_{T}+ \\
+\vec{K} M_{o}\left(\rho_{w}-\rho_{o}\right) \vec{G}
\end{array}\right)
$$




$$
f=\frac{M_{w}}{M_{o}+M_{w}}
$$

A equação (5.19) representa o fluxo fracional, também obtido na equação (2.15). Sendo assim,

$$
V_{w}=f\left(V_{T}+\vec{K} M_{o} \nabla P_{c}+\vec{K} M_{o}\left(\rho_{w}-\rho_{o}\right) \vec{G}\right)
$$

A equação (5.20) revela o campo de velocidade da fase água, dada pela relação entre a lei de Darcy e a relação de capilaridade. A equação de saturação é obtida combinando a equação (5.3) com as hipóteses (3) e (4), ou seja, a densidade não varia nem com o espaço nem com o tempo e também a porosidade varia apenas com as coordenadas espaciais. Assim sendo,

$$
\Phi(x) \rho_{\alpha} \frac{\partial}{\partial t}\left(U_{\alpha}\right)+\rho_{\alpha} \nabla \bullet\left(\vec{V}_{\alpha}\right)=q_{\alpha}
$$

Dividindo ambos os membros pela densidade $\rho_{\alpha}$, tem-se,

$$
\Phi(x) \frac{\partial}{\partial t}\left(U_{\alpha}\right)+\nabla \bullet\left(\vec{V}_{\alpha}\right)=\frac{q_{\alpha}}{\rho_{\alpha}}
$$

Uma das formas de encontrar a equação geral que governa um campo de saturação num meio poroso heterogêneo é substituir a equação (5.20) na equação (5.22). Além disso, o objetivo desse modelo é analisar e avaliar o campo de saturação da fase água, utilizando os conceitos do modelo de Corey (Brooks e Corey, 1964), discutido no capítulo 2. Em vista disso, pode-se reescrever a equação (5.22), como:

$$
\begin{aligned}
& \Phi(x) \frac{\partial}{\partial t}\left(U_{w}\right)+ \\
& \nabla \bullet\left(f_{w}\left(V_{T}+\vec{K} M_{o} \nabla P_{c}+\vec{K} M_{o}\left(\rho_{w}-\rho_{o}\right) \vec{G}\right)\right) \\
& =\frac{q_{w}}{\rho_{w}}
\end{aligned}
$$


Portanto, expandindo $\nabla P_{c}=\frac{\partial P_{c}}{\partial U_{w}} \nabla U_{w}$, obtém-se:

$$
\begin{aligned}
& \Phi(x) \frac{\partial}{\partial t}\left(U_{w}\right)+ \\
& \nabla \bullet\left(f_{w} V_{T}\right)+\nabla \bullet\left(f_{w} \vec{K} M_{o} \frac{\partial P_{c}}{\partial U_{w}} \nabla U_{w}\right) \\
& +\nabla \bullet\left(f_{w} \vec{K} M_{o}\left(\rho_{w}-\rho_{o}\right) \vec{G}\right) \\
& =\frac{q_{w}}{\rho_{w}}
\end{aligned}
$$

Lembrando que, $\vec{G}=-\vec{g} \nabla$ Depth, sendo $\vec{g}$ a aceleração gravitacional e também a operação vetorial, onde que:

$$
\nabla \bullet(A \vec{B})=A \nabla \bullet \vec{B}+\vec{B} \bullet \nabla A
$$

Para facilitar e simplificar o uso da equação (5.24) definem-se agora alguns termos, como por exemplo,

$$
\text { Gama }
$$

O sinal negativo na expressão do Gama _Flow é pelo motivo de guardar o sinal positivo uma vez que a função de pressão capilar $P_{c}$, seja uma função que diminui com saturação da água. Por isso, define-se também,

$$
\text { Gravity_Flow }=f_{w} M_{o}\left(\rho_{w}-\rho_{o}\right) \vec{g}
$$

Então, simplificando a equação (5.24), usando as equações (5.26) e (5.27), tem-se:

$$
\begin{aligned}
& \Phi(x) \frac{\partial}{\partial t}\left(U_{\alpha}\right)+ \\
& \nabla \bullet\left(f_{w} V_{T}\right)-\nabla \bullet\left(\text { Gama _Flow } \vec{K} \nabla U_{w}\right) \\
& -\nabla \bullet(\text { Gravity _Flow } \vec{K} \nabla \text { Depth }) \\
& =\frac{q_{w}}{\rho_{w}}
\end{aligned}
$$


$\mathrm{Na}$ equação (5.28) que, $f_{w} V_{T}$ representa a força viscosa, Gama_Flow $\vec{K} \nabla U_{w}$ a força de capilaridade e Gravity _ Flow $\vec{K} \nabla$ Depth , a força de gravidade. De acordo com a operação vetorial dada na equação (5.25) é possível dizer que:

$$
\left\{\begin{array}{l}
\nabla \bullet\left(f_{w} \vec{V}_{T}\right)=f_{w} \nabla \bullet \vec{V}_{T}+\vec{V}_{T} \bullet \nabla f_{w}=\vec{V}_{T} \nabla f_{w} \\
\nabla \bullet \vec{V}_{T}=0
\end{array}\right.
$$

De acordo com a hipótese (3). Acrescenta-se ainda:

$$
\left\{\begin{array}{l}
\nabla \bullet\left(\text { Gama_Flow } \vec{K} \nabla U_{w}\right)= \\
\text { Gama_Flow } \nabla \bullet\left(\vec{K} \nabla U_{w}\right)+ \\
\vec{K} \nabla U_{w} \nabla(\text { Gama_Flow })
\end{array}\right.
$$

Finalmente,

$$
\left\{\begin{array}{l}
\nabla \bullet(\text { Gravity_Flow } \vec{K} \nabla \text { Depth })= \\
\text { Gravity_Flow } \nabla \bullet(\vec{K} \nabla \text { Depth })+ \\
\vec{K} \nabla \text { Depth } \nabla(\text { Gravity _ Flow })
\end{array}\right.
$$

Diante das equações (5.29), (5.30) e (5.31), a equação (5.28), pode ser reescrita na forma simplificada:

$$
\begin{aligned}
& \Phi(x) \frac{\partial}{\partial t}\left(U_{\alpha}\right)+\vec{V}_{T} \nabla f_{w}= \\
& \frac{q_{w}}{\rho_{w}}+\text { Gama_Flow } \nabla \bullet\left(\vec{K} \nabla U_{w}\right)+ \\
& \vec{K} \nabla U_{w} \nabla\left(\text { Gama } \_ \text {Flow }\right)+ \\
& \text { Gravity_Flow } \nabla \bullet(\vec{K} \nabla \text { Depth })+ \\
& \vec{K} \nabla \text { Depth } \nabla(\text { Gravity _ Flow })
\end{aligned}
$$

Constata que a equação (5.32), é uma equação de meios porosos heterogêneos, para determinar o campo de saturação, considerando os efeitos viscosos, efeitos de capilaridade e os efeitos gravitacionais. Também se pode dizer que é uma equação de Buckley-Leverett na sua forma geral, considerando as forças viscosas, forças capilares e as forças gravitacionais. Em vista disso, a 
equação (5.32) será utilizada nesta pesquisa para achar o campo de saturação em todo o domínio para todos os instantes de tempo. Por simplicidade será considerado um caso num meio poroso heterogêneo onde os efeitos gravitacionais são desprezíveis.

Para facilitar o entendimento do algoritmo proposto à frente serão definidos alguns outros termos, como:

$$
\begin{aligned}
& \text { Capillary_Effects }= \\
& \text { Gama_Flow } \nabla \bullet\left(\vec{K} \nabla U_{w}\right)+ \\
& \vec{K} \nabla U_{w} \nabla(\text { Gama__Flow })
\end{aligned}
$$

Bem como:

$$
\begin{aligned}
& \text { Gravity_Effects }= \\
& \text { Gravity_Flow } \nabla \bullet(\vec{K} \nabla \text { Depth })+ \\
& \vec{K} \nabla \text { Depth } \nabla(\text { Gravity _ Flow })
\end{aligned}
$$

Para um maior detalhamento dos métodos e modelagens utilizados nesta pesquisa, assim como a descrição completa da modelagem de fluxo de fluidos em meios porosos num reservatório podem ser consultadas as obras e trabalhos gerais dos seguintes autores: Peaceman (1977), Aziz e Settari (1979), Ewing (1983), Rosa et al (2006) e Hasle et al (2007).

A seguir serão discutidas formas e estratégias de representar as equações para o cálculo de pressão-velocidade (equação 5.14) e equações para tratamento do campo de saturação (equação 5.32). O objetivo principal é adaptar essas equações aos métodos propostos nos capítulos (3) e (4).

\section{2.}

\section{Estratégias para representação do modelo proposto}

Nesta seção serão estudadas técnicas para representação das equações para aproximar o upstream point, a equação de pressão-velocidade e a equação da saturação. A ideia principal é usar a formulação Semi-Lagrangeana baseada no método característico, que tem sido largamente utilizada para resolver problemas 
de advecção no transporte de uma grandeza escalar, neste caso o campo de saturação.

Nos capítulos anteriores discutiu-se sobre a derivada material ou advecção, e ainda o método característico na sua forma clássica, que encerram o mesmo conceito. Como agora será considerado um meio poroso heterogêneo, para advectar o campo de saturação na equação do mesmo aparecerá um novo termo da propriedade da rocha conhecido como porosidade. O artificio utilizado incluindo o termo de porosidade na advecção é adaptar pequenas modificações no método caraterístico, que serão analisadas e estudadas mais a frente durante o capítulo. Por agora pode-se dizer que essas modificações foram propostas principalmente por Douglas e Russell (1982) e Zhu et el (2014). Portanto, a equação de derivada material ou a advecção na sua forma modificada foi proposta da seguinte forma:

$$
\frac{D}{D t}=\frac{\partial}{\partial t}+\frac{\vec{V}_{T}}{\Phi(x)} \nabla
$$

Ou seja, ainda pode ser escrita na forma de diferenciação numérica, em relação ao campo de saturação,

$$
\begin{aligned}
& \Phi(x) \frac{D\left(U\left(x, t^{m+1}\right)\right)}{D t}= \\
& \Phi(x) \frac{U\left(x, t^{m+1}\right)-U\left(\tilde{x}, t^{m}\right)}{\Delta t}
\end{aligned}
$$

Note que, o upstream point agora será dado, por:

$$
\tilde{x}=x-\frac{V_{T}\left(x, t^{m+1}\right) \Delta t}{\Phi(x)}
$$

Observe que a advecção na sua forma modificada, na equação (5.35), o campo de velocidade está sendo dividido pela porosidade $\Phi(x)$, razão pelo qual justifica o aparecimento da porosidade também na equação (5.37). Logo, no primeiro membro da equação (5.32) pode-se dizer que:

$$
\begin{aligned}
& \Phi(x) \frac{U\left(x, t^{m+1}\right)-U\left(\tilde{x}, t^{m}\right)}{\Delta t}= \\
& \Phi(x) \frac{\partial}{\partial t}\left(U_{\alpha}\right)+\vec{V}_{T} \nabla f_{w}
\end{aligned}
$$


Então, a equação (5.32) pode ser reescrita usando a ideia de advecção modificada, como:

$$
\begin{aligned}
& \Phi(x)\left(\frac{U\left(x, t^{m+1}\right)-U\left(\tilde{x}, t^{m}\right)}{\Delta t}\right)= \\
& \frac{q_{w}}{\rho_{w}}+\text { Gama_Flow } \nabla \bullet\left(\vec{K} \nabla U_{w}\right)+ \\
& \vec{K} \nabla U_{w} \nabla(\text { Gama_Flow })+ \\
& \text { Gravity__Flow } \nabla \bullet(\vec{K} \nabla \text { Depth })+ \\
& \vec{K} \nabla \text { Depth } \nabla(\text { Gravity _ Flow })
\end{aligned}
$$

Por fim,

$$
U\left(x, t^{m+1}\right)=U\left(\tilde{x}, t^{m}\right)+\frac{\Delta t}{\Phi(x)}\left(\begin{array}{l}
\frac{q_{w}}{\rho_{w}} \\
\text { Gama_Flow } \nabla \bullet\left(\vec{K} \nabla U_{w}\right)+ \\
\vec{K} \nabla U_{w} \nabla(\text { Gama_Flow })+ \\
\text { Gravity_Flow } \nabla \bullet(\vec{K} \nabla \text { Depth })+ \\
\vec{K} \nabla \text { Depth } \nabla(\text { Gravity _Flow })
\end{array}\right)
$$

A equação (5.40) representa o campo de saturação utilizando o método característico modificado, em um meio poroso heterogêneo, considerando os efeitos viscosos, efeitos de capilaridade e efeitos de gravidade.

A seguir será apresentado um novo algoritmo adaptativo baseado nessa ideia junto com os métodos de aproximação meshfree num meio poroso heterogêneo.

\subsection{1}

Novo algoritmo adaptativo para simulação de dois fluidos imiscíveis e incompressíveis em meios porosos heterogêneos.

Como mencionado no algoritmo apresentado na seção (3.2), que para cada nó $\xi$ do conjunto $\Xi \equiv \Xi^{t}$ (para o tempo $t$ ), uma aproximação do ponto $x$ 
correspondente ao upstream point $\tilde{x}$, agora é computada pela equação (5.37). Repare que agora haverá uma atualização do campo de pressão-velocidade através da variação do campo de saturação, que por sua vez, será atualizada também pela variação da mobilidade das fases para cada instante de tempo.

As concentrações de saturações $U(t, x)$ do upstream point $\tilde{x}$ são determinadas usando a equação (5.40). Nesta equação são considerados os efeitos das forças. Além disso, as aproximações da saturação $U\left(\tilde{x}, t^{m}\right)$ e da velocidade do upstream point $\tilde{x}$ são dadas pela interpolação local usando splines poliharmônicas, através do conjunto dos vizinhos do mesmo ponto.

Para determinar todos os valores da saturação para todos instantes de tempo, $U\left(\xi, t^{m+1}\right)$, dados pela equação (5.40), cada nó $\xi \in \Xi$ será ou não refinado ou simplificado de acordo com a avaliação do erro gerado pela interpolação spline poliharmônica. Após várias iterações, refinando e simplificando o modelo, haverá uma estabilidade na correção do valor de saturação para cada nó, o que implicará na obtenção de um novo conjunto de nós, $\Xi \equiv \Xi^{t+\tau}$, para ser usado no instante de tempo subsequente. A simplificação ou remoção do nó depende de regras propostas baseadas no erro gerado, $\eta(\xi), \xi \in \Xi$ pela interpolação local em volta do nó $\xi$, como mencionado anteriormente.

Será agora, discutido o novo algoritmo adaptativo para simulação de dois fluidos imiscíveis e incompressíveis em meios porosos heterogêneos, de forma mais detalhada.

\section{Dados entrada}

Os valores que foram usados como dados de entrada são:

- O domínio que pode ser utilizando uma malha fixa ou meshfree.

- Descrição do reservatório, para o problema de five spot.

○ Posição dos poços;

○ Permeabilidade relativa e absoluta;

○ Porosidade;

○ Pressão inicial;

○ Razão inicial de injeção e produção; e

- Profundidade dos poços. 
- Número de passos de tempo.

- Discretização do tempo, delta T.

\section{Condições iniciais e de contorno}

- Relação de pressão inicial do reservatório.

- Profundidade inicial do reservatório.

- Uso de modelo Corey (Brooks e Corey, 1964), para determinação das permeabilidades relativas da água e do óleo.

- Uso do modelo Van Genuchten, mencionado no capítulo 2, para determinação da variação de pressão e de capilaridade em relação à saturação da água na equação (5.26) para determinar o Gama _ Flow (Marshall, 2009).

- Saturação inicial que é dada pela relação entre a saturação da água e a saturação do óleo, lembrando que a soma dos dois é igual a 1.

\section{Dados de saída}

- Saturação $U\left(\xi, t^{m+1}\right)$ em cada instante de tempo.

- Pressão e Velocidade em cada instante de tempo

- Os resultados podem ser considerados os efeitos gravitacionais ou não.

A seguir será apresentado o algoritmo propriamente dito: 
1. Para cada instante de tempo faça: $t \rightarrow t+\tau$

1.1- Atualizar as permeabilidades relativas da água e do óleo usando o modelo de Corey (Brooks e Corey, 1964), mencionado no capítulo 2.

1.2- Atualizar as mobilidades da água e do óleo usando as novas permeabilidades relativas. É possível obter o fluxo fracional nesta etapa através do novo campo de mobilidades.

1.3- Atualizar o campo de pressão - velocidade através da equação (5.14) ou (5.20) usando também o novo campo das mobilidades da água e do óleo.

1.4- Atualizar os efeitos de capilaridade e efeitos gravitacionais dados das equações (5.33) e (5.34), caso esses efeitos forem consideradas.

1.5- Iterar até a saturação do conjunto dos nós ficar estabilizada

1.5.1 - Para cada ponto randômico $\xi \in \Xi$ faça:

1.5.1.1 - Calcule nova posição de $x \_u p s t r e a m$, agora dada pela equação (5.37), usando aproximação por iteração de ponto fixo.

1.5.1.2 - Compute os vizinhos do $x \_u p s t r e a m$ usando a ideia de distancia mínima.

1.5.1.3 - Compute a saturação do $x \_u p s t r e a m$ dada pela $R B F$ interpolação local, splines poliharmônicas, $U\left(\tilde{x}, t^{m}\right)$.

1.5.1.4 - Advecte a saturação já interpolada, pela equação (5.40).

1.5.1.5 - Determine o erro máximo entre o valor da saturação $U$ do $x \_$upstream e o valor da Saturação advectado, $\eta(\xi)=\left|U\left(x, t^{m+1}\right)-U\left(\tilde{x}, t^{m}\right)\right|$. O erro máximo é dado por, ErroMax $=\max (\eta(\xi))$.

\subsubsection{6 - Fim da etapa 1.5.1}

1.5.2 Refinamento/Simplificação, $\Xi \equiv \Xi^{t+\tau}$;

- Se o Erro de cada nó $\xi \in \Xi$ for menor do que $\theta_{\text {coarse }} *$ ErroMax então, remova $U$.

- Se o Erro de cada nó $\xi \in \Xi$ for maior que $\theta_{\text {refine }} *$ ErroMax, refina, ou seja, insira novos pontos Voronoi e retorna ao passo 1.5 a 1.5 .3 novamente.

- Senão a saturação $U=U\left(x, t^{m+1}\right)$ obtida no passo 1.5.1.4.

\subsubsection{Fim da etapa 1.5.}

1.5.4 Armazena todos os valores da saturação $U$ para cada instante de tempo, onde agora $\Xi \equiv \Xi^{t+\tau}$.

\subsubsection{Fim da etapa 1.}

2. Imprimir Resultados finais de $\xi \in \Xi$ do campo de saturação $U\left(x, t^{m+1}\right)$, para todos os instantes de tempo.

3. Fim do Programa 
Como mencionado anteriormente, este algoritmo necessita armazenar dois diferentes conjuntos de nós, um no instante $t$ (com valores conhecidos em locais conhecidos), e outro no instante $t \rightarrow t+\tau$, (valores e posições desconhecidos), que inicialmente são os mesmos, e que vai sendo gradativamente atualizado. A grande diferença entre o modelo anterior proposto por Iske e Käser (2005) e o novo modelo adaptado é que este foi generalizado para ser aplicado a um problema mais geral, utilizando um meio poroso heterogêneo. Em outras palavras, a variação das propriedades heterogêneas, isto é, da rocha, como as permeabilidades e a porosidade influenciarão no calculo e no comportamento físico do upstream point, como também no campo de pressão, velocidade e saturação.

Importante salientar que o algoritmo sugerido nesta seção (passo 1.1) sempre precisará atualizar o campo das permeabilidades relativas de ambas as fases. Esta atualização é realizada pela variação de campo de saturação em cada instante de tempo, baseado no modelo de Corey. As mobilidades de ambas as fases se atualizarão pela atualização das permeabilidades relativas dos mesmos (passo 1.2), do mesmo modo, que a atualização do campo de pressão e velocidade (no passo 1.3). A consideração ou não dos efeitos capilares e/ou gravitacionais influenciarão na atualização dos mesmos, no passo 1.4.

O passo (1.5.1.1) calcula a trajetória a nova posição dos nós utilizando a aproximação do upstream point, que é dada pela equação (3.12). A modificação será feita no calculo de $\beta$. Tendo em vista que se trata de um meio poroso heterogêneo, deve-se levar em conta, também, a porosidade do meio, tomando $\beta$ como.

$$
\beta=\tau \cdot \frac{\vec{V}}{\Phi(x)}
$$

Agora a trajetória da partícula é acompanhada pela variação do campo de pressão, velocidade e saturação. Como explicado anteriormente na seção 5.1, para um caso de advecção passiva (caso linear), a velocidade é dada por $\vec{V}=v$. Entretanto, para um caso não linear a velocidade de advecção é dada pela variação, principalmente, das mobilidades de ambas as fases que variam de acordo com a variação das permeabilidades relativas dos mesmos. 
A aproximação de $\beta$, é obtida utilizando aproximação por fixed point iteration, baseado na ideia de midpoint e também no método característico modificado (Douglas e Russel, 1982). Da mesma forma que, para computar $V\left(t+\frac{\tau}{2}, \xi-\frac{\beta^{k}}{2}\right)$, será utilizado o esquema de extrapolação dada pela equação (3.15). Assim, após ter determinando o upstream point, é possível advectá-lo, determinando a saturação do mesmo através da equação (5.40). Logo, nos passos 1.5.1.2, 1.5.1.3, 1.5.1.4, determinam-se os vizinhos do valor de upstream point aproximado, a interpolação por spline poliharmônica e por fim a advecção da saturação $U$, para um tempo $t+\tau$, dado pela equação (5.40), variando de acordo com o caso. Além disso, no passo 1.5.1.5, computa-se o erro máximo local. Este erro é dado pela diferença do valor da saturação do upstream point advectado menos o valor da saturação $U$ aproximado, calculado pela interpolação por spline poliharmônica, isto é $\eta(\xi)=\left|U\left(x, t^{m+1}\right)-U\left(\tilde{x}, t^{m}\right)\right|$. Este erro indicará se deve haver simplificação ou refinamento.

Finalmente, o processo de refinamento ou simplificação dos nós é executado com base no erro máximo calculado no passo 1.5.1.5. Os valores $\theta_{\text {refine }}$ e $\theta_{\text {coarse }}$, onde $0 \leq \theta_{\text {coarse }}<\theta_{\text {refine }} \leq 1$, servem para definir quando deve haver refinamento ou simplificação. Com isto tem-se um novo conjunto de nós com novas informações de pressão, velocidade e saturação. De posse deste novo conjunto de nós o algoritmo retorna para o passo (1.5) e os passos 1.5.1 a 1.5.2 se repetem até não encontrar mais regiões nem para refinar nem para remover, isto é, quando a iteração converge.

No próximo capítulo serão apresentados todos os resultados obtidos a partir do modelo proposto, incluindo geometrias unidimensional, bidimensional e tridimensional. 


\section{6 Resultados}

Sumarizando os capítulos anteriores, foram descritos e discutidos os métodos numéricos como o método Level Set, o método Semi-Lagrangeano, os métodos meshfree, assim como o método de Kansa e o método de Hermite. Também foram apresentadas as inserções de algumas hipóteses, como condições iniciais e condição de fronteira de Dirichlet. Os métodos propostos não possuem solução analítica, salvo em casos muito simplificados. $\mathrm{O}$ acoplamento desses métodos tem por finalidade uma boa convergência e funcionamento do modelo simulado, extraindo a melhor aplicação de cada um deles. Este capítulo propõe uma análise dos resultados obtidos de cada método proposto, separadamente e também dos resultados combinando-os, tendo em vista os objetivos propostos inicialmente neste trabalho.

Neste capitulo são exibidos alguns resultados alcançados e também ponderações observadas. Em primeiro lugar, são apresentados alguns resultados do método Semi-Lagrangeano. Em são apresentados alguns resultados do modelo meshfree adaptativo para o problema bidimensional e na sequência alguns resultados do novo modelo meshfree adaptativo de advecção para problemas de fluxo bifásico e de fluidos incompressíveis e imiscíveis em meios porosos heterogêneos tridimensionais.

\section{1. \\ Método Semi-Lagrangeano $1 D$ e $2 D$}

Para testar e analisar o método Semi-Lagrangeano foram resolvidos dois problemas numéricos, usando a equação de advecção linear e não linear. O primeiro é um problema $1 D$ baseado na propagação de onda senoidal linear, com velocidade constante ao longo do tempo, feito pelos autores (Costa et al, 2004). Os mesmos autores utilizaram o método de diferenças finitas para aproximar as derivadas parciais da equação diferencial. O segundo é um problema em $2 D$ que pode ser entendido como um modelo simples de reservatório de petróleo clássico 
conhecido como five spot, também com velocidade constante ao longo tempo (Iske e Käser, 2005).

O modelo five spot (também conhecido por modelo de malha de cinco pontos) é um esquema de injeção no qual os poços (tanto os de injeção como os de produção) estão uniformemente distribuídos em toda a área do reservatório. Neste caso, o fluido deslocante é injetado na zona de óleo, o que altera a distribuição da saturação e o movimento dos fluidos. Este tipo de modelo é recomendado para reservatórios com grandes áreas e pequenas inclinações e espessuras (Rosa et al. 2006).

\subsection{1.}

\section{Modelo 1D}

O modelo $1 D$ simulado nesta pesquisa, acima mencionado, foi a propagação de uma onda senoidal linear com velocidade constante ao longo do tempo. Nesta tese utilizou-se a ideia do método Semi-Lagrangeano para representar o mesmo problema $1 D$. Considere os seguintes dados do problema:

$$
U_{t}+C(x) U_{x}=0, \quad C(x)=\frac{1}{5}+\operatorname{sen}^{2}(x-1)
$$

Onde $U_{t}$ e $U_{x}$ são as variações temporais e espaciais do campo escalar $U$, ainda $C(x)$ é o campo de velocidade, neste caso, independentemente do tempo. As condições iniciais e de contorno são dadas abaixo:

$$
0 \leq x \leq 2 \pi, \quad 0 \leq t \leq 8
$$

A equação (6.1) é uma equação de advecção com coeficiente variável espacialmente. A seguir serão apresentados os resultados obtidos pelos autores (Costa et al, 2004), em comparação com os resultados obtidos usando o método Semi-Lagrangeano. Os autores utilizaram uma malha unidirecional com 128 pontos. 


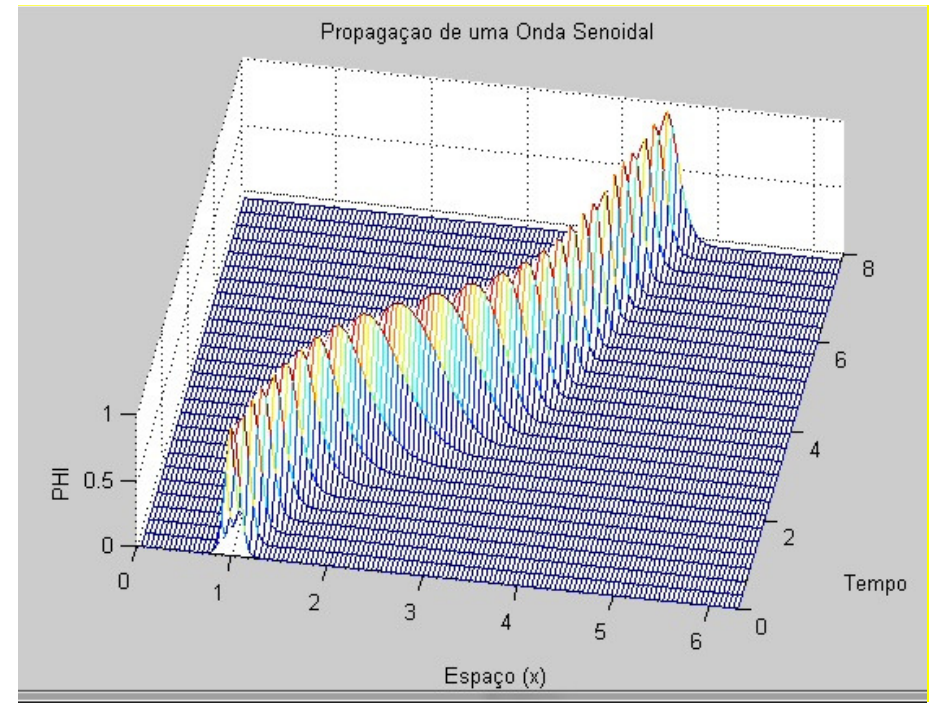

Figura 22: Propagação de uma Onda Senoidal Unidirecional (Costa et at, 2004)

A Figura 22 exibe a solução da equação de advecção de coeficiente variável obtida pelos autores (Costa et al, 2004). Os mesmos autores consideraram que o coeficiente $C(x)$ é sempre positivo, então a elevação se move para direita com o tempo, conforme mostra a Figura 22. A Figura 23, produzida por este trabalho, mostra o mesmo problema $1 D$ usando a abordagem SemiLagrangeana.

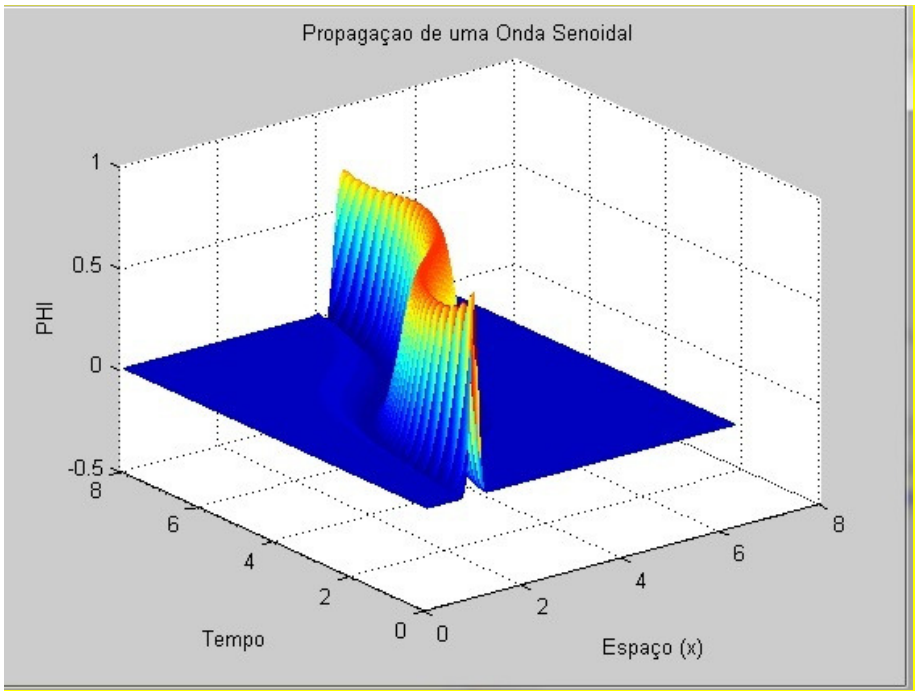

Figura 23: Propagação de uma Onda Senoidal Unidirecional utilizando o método SemiLagrangeano 
Note-se que a propagação da onda senoidal é constante ao longo do tempo, isto é, a velocidade é constante ao longo do tempo, variando apenas espacialmente, como no problema original. Como pode ser observado na Figura 22 e na Figura 23 o resultado obtido é exatamente o mesmo validando assim a implementação $1 D$ do método Semi-Lagrangeano.

\subsection{2.}

\section{Modelo 2D}

O modelo $2 D$ é um problema clássico de meios porosos não linear de reservatório de petróleo conhecido como five spot, como mencionado anteriormente, o modelo se baseia na equação de advecção de Buckley-Leverett viscoso, equação (3.5) (Buckley e Leverett, 1942). A ideia principal desde modelo $2 D$ é calcular para cada instante de tempo a saturação $U$ em todos os upstream points através da interpolação local. Neste caso, utilizou-se a interpolação local por splines poliharmônicas (Iske, 2003), discutida na secção (3.3).

O domínio computacional é dado como, $\Omega=[-0.5,0.5]^{2}$, que corresponde à limitação do reservatório em ambas as direções. Inicialmente os poros do reservatório estão saturados com o fluido óleo, quando a saturação $U_{o}=0$ e com o fluido água, quando a saturação $U_{w}=1$, isto é, inicialmente no centro do poço o raio de injeção $R$ contêm o fluido água e fora do raio de injeção $R$ contêm o fluido óleo, conforme mostra a equação abaixo:

$$
U= \begin{cases}1 & \text { para }\|x-o\| \leq R \\ 0 & \text { outros casos }\end{cases}
$$

Onde raio de injeção $R=0.02$ e centro $o=(0,0)$, ambos pertencentes ao domínio $\Omega$. A posição dos outros quatro cantos dos poços sugeridos pelos autores são:

$$
C=\left\{\begin{array}{ll}
(-0.5,-0.5), & (-0.5,0.5) \\
(0.5,-0.5), & (0.5,0.5)
\end{array}\right\}
$$


Na equação (6.4), $C$ representa os poços de produção do reservatório. Os balanços de massa de ambos os fluidos são dados pelas equações (6.5) e (6.6).

$$
\begin{aligned}
& \Phi(x) \frac{\partial}{\partial t} U_{w}(t, x)+\nabla \bullet \vec{V}_{w}(t, x)=0 \\
& \Phi(x) \frac{\partial}{\partial t} U_{o}(t, x)+\nabla \bullet \vec{V}_{o}(t, x)=0
\end{aligned}
$$

A equação (6.5) representa a conservação da massa da água e $\vec{V}_{w}(t, x)$ é a velocidade de fase da água ao longo do tempo. Analogamente, a equação (6.6) se refere à conservação da massa do óleo e $\vec{V}_{o}(t, x)$ é a velocidade de fase do óleo ao longo do tempo. Em ambas as equações (6.5) e (6.6), $\Phi(x)$ denota a porosidade do meio, que determina o volume do fluxo do fluido. Assim, para qualquer meio homogêneo, sua porosidade $\Phi(x)$ é constante (Iske e Käser, 2005). $U_{w}$ e $U_{o}$ são as frações de volume da água e do óleo no meio poroso. Portanto, a relação do balanço de massa entre as fases água e óleo é dada por:

$$
U_{w}(t, x)+U_{o}(t, x)=1
$$

Visto que o meio ou o domínio é constituído por apenas duas fases, isto é, bifásico. Os dois campos de velocidade das duas fases, $\vec{V}_{o}(t, x)$ e $\vec{V}_{w}(t, x)$ são determinados pela Lei de Darcy na sua forma simplificada (equação 2.11) (Darcy, 1856), como se segue:

$$
\vec{V}_{w}(t, x)=-\vec{K}(x) M_{w}\left(U_{w}\right) \nabla P(t, x)
$$

$\mathrm{e}$

$$
\vec{V}_{o}(t, x)=-\vec{K}(x) M_{o}\left(U_{o}\right) \nabla P(t, x)
$$

Onde $P(t, x)$ é a pressão do reservatório e $\vec{K}(x)$ é o tensor de permeabilidades do meio poroso. $M_{w}\left(U_{w}\right)$ e $M_{o}\left(U_{o}\right)$ são as mobilidades das fases água e óleo dada na equação (2.14), segundo o modelo de Brooks e Corey (1964) apresentado na seção 
2.4. Ainda $k_{w}\left(U_{w}\right)=U^{2}, k_{o}\left(U_{o}\right)=(1-U)^{2}$, são as permeabilidades relativas das fases água e óleo, também utilizando o modelo de Brooks e Corey (1964) que são idênticas às equações (2.13). Lembrado que $\mu_{w}$ e $\mu_{o}$, são as viscosidades das fases correspondentes.

Considerando a hipótese de que o escoamento seja incompressível (equação 2.4), e também combinando as equações de conservação de massa de ambos as fases água e óleo (equações 6.5 e 6.6), obtém-se a seguinte relação:

$$
\nabla \bullet\left(\vec{V}_{w}(t, x)+\vec{V}_{o}(t, x)\right)=\nabla \bullet \vec{V}(t, x)=0
$$

Comprovando que a velocidade total do fluido na relação de incompressibilidade é estacionária, ou seja, a relação mostra que o divergente da velocidade total é igual a zero. Note que a equação (6.10) é idêntica a equação (2.4).

Ainda, segundo os autores (Iske e Käser, 2005), se a mobilidade for $M \equiv 1$, as equações (6.8 e 6.9) satisfazem a condição de incompressibilidade, sendo assim, as equações (6.8 e 6.9) podem ser reescritas na forma:

$$
\vec{V}=-\nabla P
$$

E isto pode se aplicar para ambas as fases. O problema de five spot proposto nesta tese considera também o campo de velocidade como estacionário, ilustrado na equação (6.11). Pela correlação pressão-velocidade, se a velocidade é estacionária então, a pressão também será. Neste modelo, o campo de pressão utilizado pelos autores Iske e Käser (2005) é:

$$
\begin{aligned}
& P(x)=\sum_{c \in C} \log (\|x-c\|)-\log (\|x-o\|), \\
& x \in \Omega \quad c \in C
\end{aligned}
$$

Portanto, o problema de five spot consiste em injetar o fluido água do centro do reservatório através do raio da injeção e o fluido óleo é empurrado pela saturação da água ao longo do tempo, para os quatros outros poços de produção. 
A seguir serão exibidos alguns resultados obtidos pelos autores (Iske e Käser, 2005), em comparação com os resultados obtidos usando o programa desenvolvido por esta tese. Durante a simulação foi usado um passo de tempo de $\tau=5 \times 10^{-5}$. De acordo com os mesmos autores um passo de tempo muito pequeno foi utilizado para evitar que os upstream points caíssem fora do domínio. Já que para este problema em particular a estimativa do passo é extremamente importante no cálculo do upstrean point, a simulação ocorre no intervalo de tempo $I=[0,2100 \tau]$ e o coeficiente de difusão é dada por $\varepsilon=0.015$.

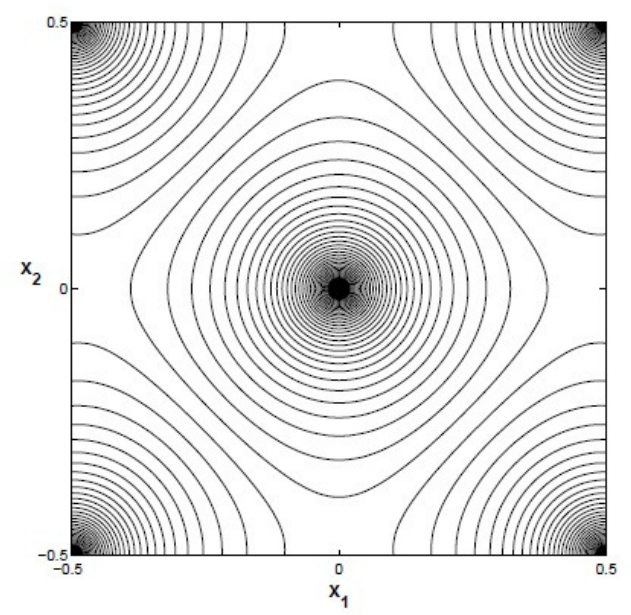

Figura 24: Campo de Pressão (Iske e Käser, 2005)

A Figura 24 exibe os contornos do campo de pressão calculado através da equação (6.12). Observa-se que a pressão é muito alta nos cinco poros do poço, ou seja, há presença de singularidades nos cantos e no centro do domínio. Há uma grande variação de pressão entre os cinco poços do domínio e na vizinhança dos mesmos, conforme apresentado na Figura 24. A pressão é pequena entre os poços, isso reflete o comportamento físico real do problema de five spot, onde existem pequenos efeitos de pressão na saturação, (Thomas, 2001). 


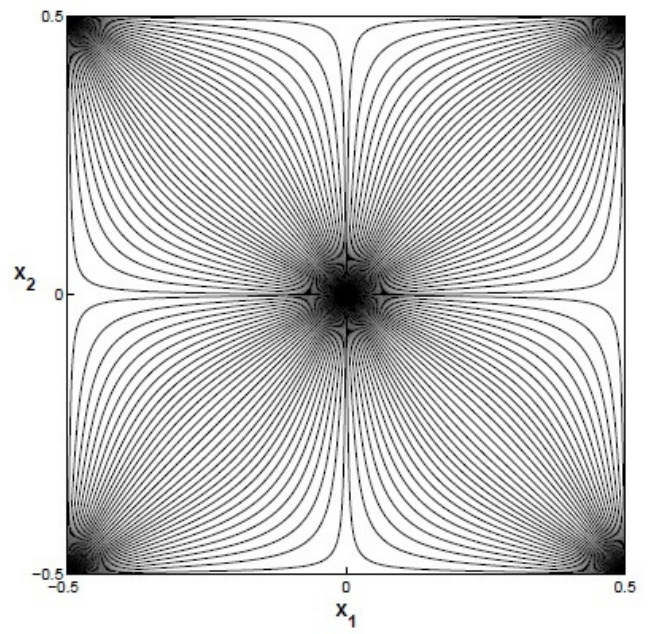

Figura 25: Campo de Velocidade(Iske e Käser, 2005)

Pela correlação pressão-velocidade, o mesmo deve acontecer com o campo de velocidade como mostra a Figura 25, aplicando a equação (6.11). Com o campo de velocidade em todo o domínio é também possível determinar o campo de Saturação em todo o domínio. A solução de saturação $U$ é dada a cada instante de tempo pela combinação das equações (3.11 e 6.10) e pela interpolação local por spline poliharmônica obtida pela equação (3.17).

A Figura 26 mostra a saturação $U$ da água em seis diferentes instantes de tempo, $t=t_{0}, t=t_{420}, t=t_{840}, t=t_{1260}, t=t_{1680}$ e $t=t_{2100}$, onde a saturação $U$ é avaliada numa malha cartesiana fixa retangular de 100 x 100 células.

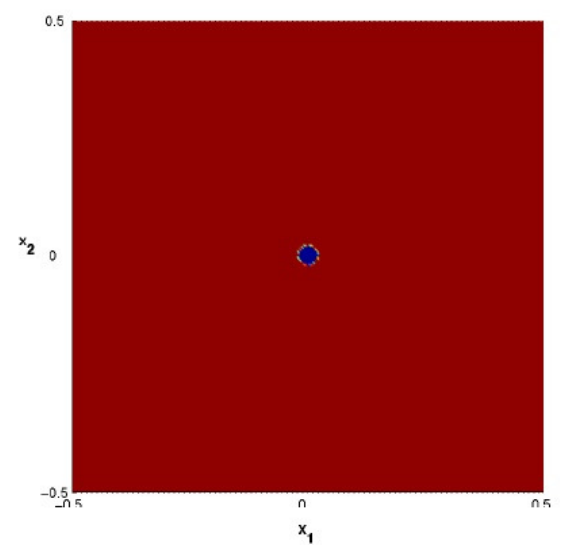

(a)

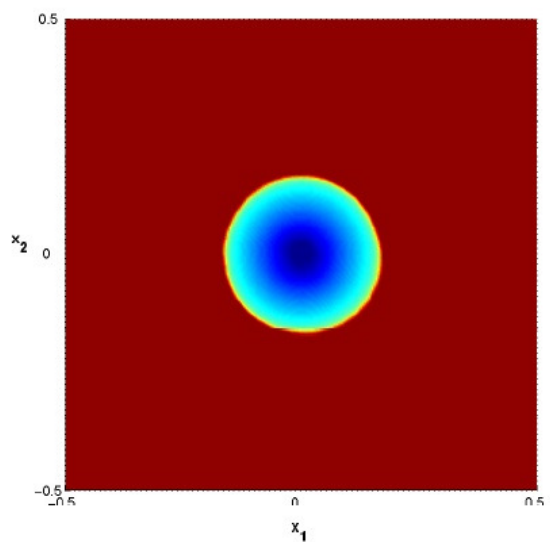

(b) 


\section{Continuação}

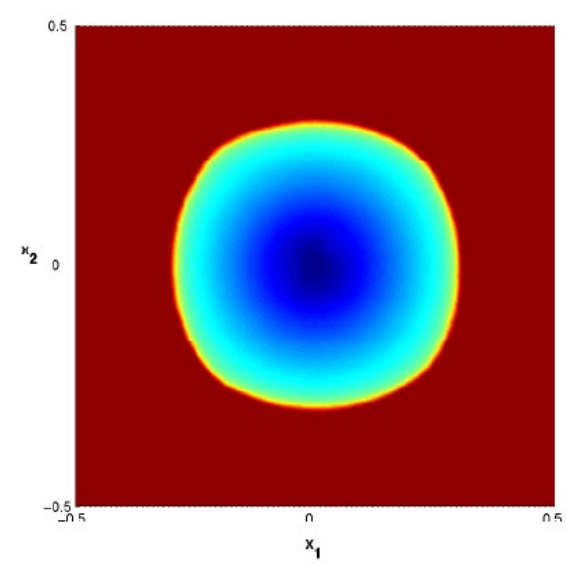

(c)

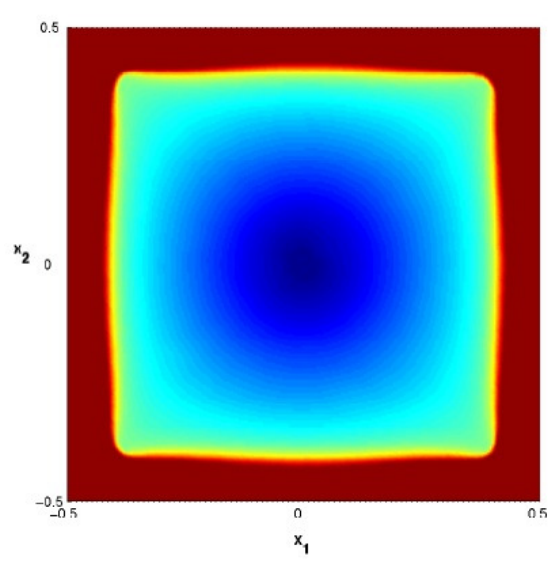

(e)

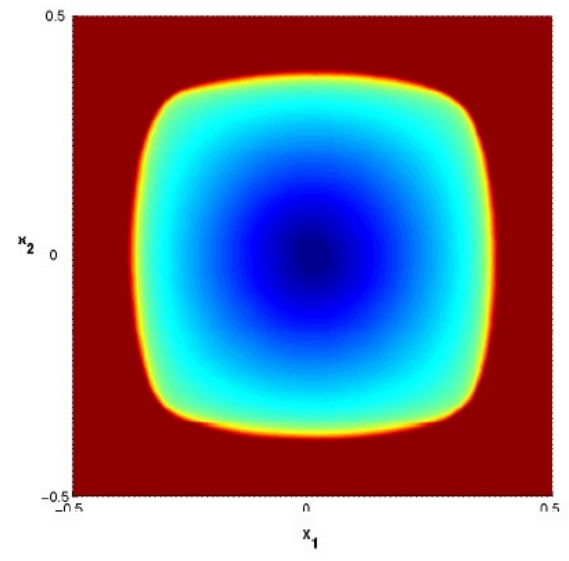

(d)

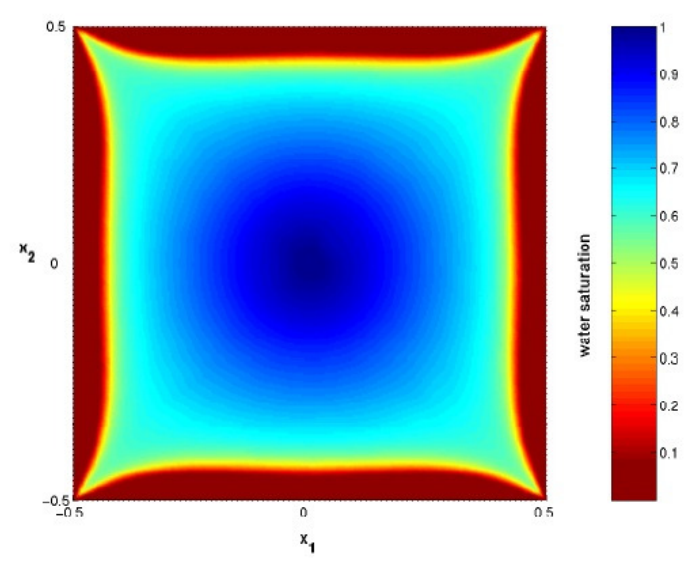

(f)

Figura 26: Saturação no problema five spot em 6 instantes de tempo (t) (Iske e Käser, 2005)

No código de cores ilustrado à direita da Figura 26, a cor vermelha representa o fluido puramente óleo, ou seja, saturação $U=0$ enquanto que a cor azul escura indica o fluido puramente água, isto é, saturação $U=1$. A combinação das cores entre vermelha e azul escuro mostra a variação da saturação $U$ da água, ao longo do tempo. Note que a interface água / óleo está se deslocando do centro para os quatro poços das extremidades.

São apresentados a seguir os resultados dos campos de pressão, velocidade e de saturação obtidos pelo programa desenvolvido nesta pesquisa, usando os 
mesmos dados propostos pelos autores Iske e Käser (2005). Da Figura 27 à Figura 30 são exibidas os principais resultados obtidos na simulação do problema $2 D$ de five spot através da combinação da equação de advecção de Buckley-Leverett viscoso proposto na equação (3.5) e na equação de incompressibilidade (6.10), usando o modelo do programa desenvolvimento nesta pesquisa.

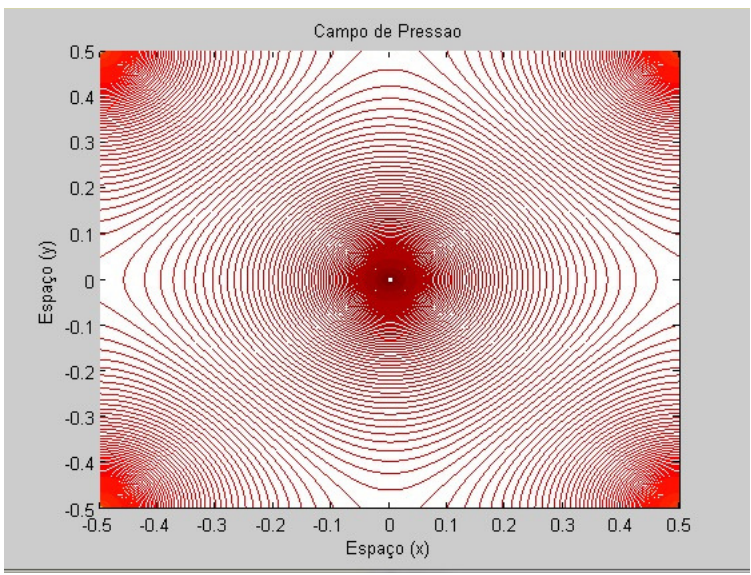

Figura 27: Campo de Pressão. Resultado obtido usando o modelo da tese

A Figura 27 ilustra o campo de pressão do problema de five spot. O mesmo também foi determinado usando a equação (6.12). Note que em comparação com Figura 24, o mesmo resultado foi alcançado, com a presença de singularidades nos quatro cantos e no centro dos poços.

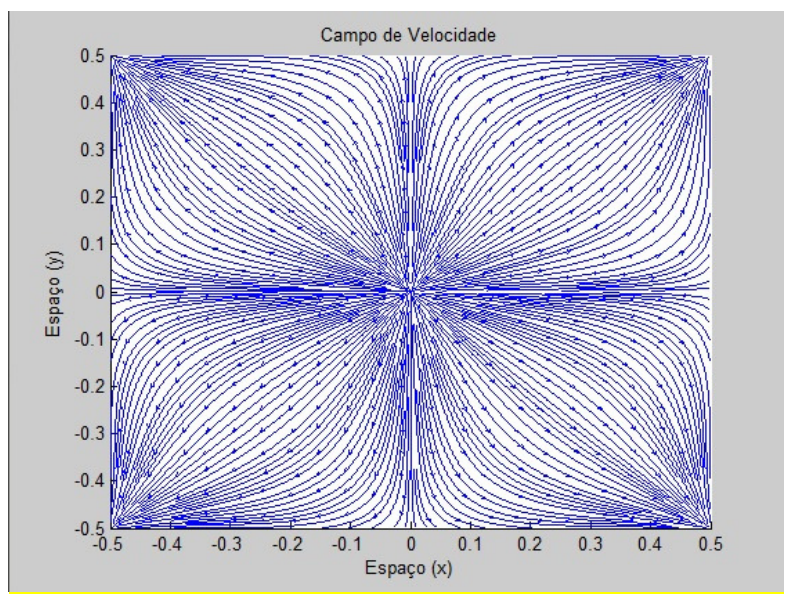

Figura 28: Campo de Velocidade. Resultado obtido usando o modelo da tese

Na Figura 28 observa-se o campo de velocidade calculado pelo programa, que foi determinado através da equação (6.11). Como esperado, o comportamento 
do campo de velocidade, devido à correlação pressão-velocidade é semelhante ao campo de pressão da Figura 24 e da Figura 25.

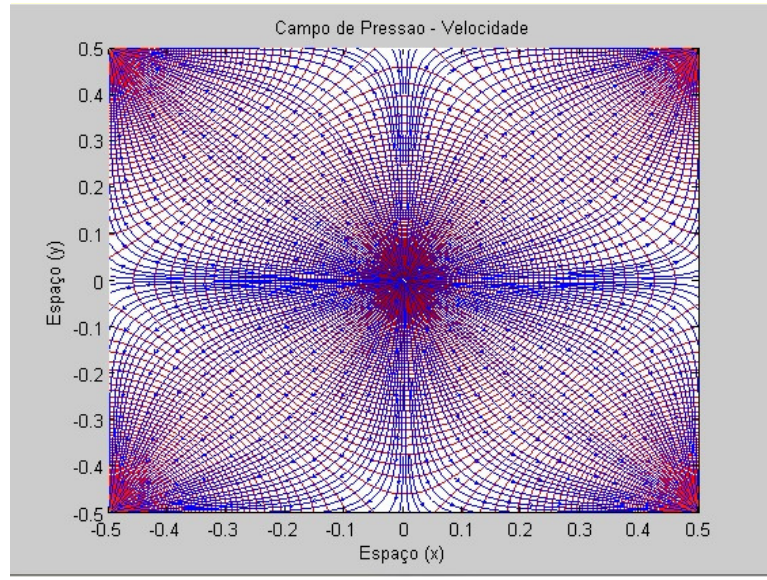

Figura 29: Campo de Pressão-Velocidade sobreposto. Resultado obtido usando o modelo da tese

Ainda explorando o modelo criado por esta pesquisa, a Figura 29 ilustra a correlação pressão-velocidade de acordo com a lei de Darcy simplificada, obtida pelas equações (6.11 e 6.12). 
Figura a: Saturacao no tempo $=0$

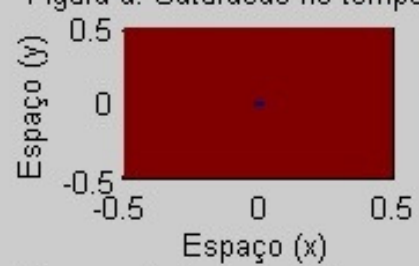

Figura c: Saturacao no tempo $=840$

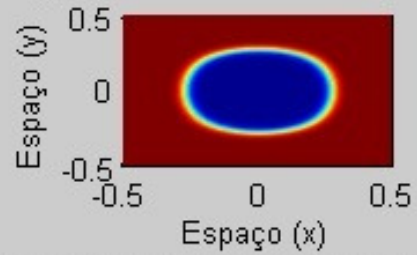

Figura e: Saturacao no tempo $=1680$

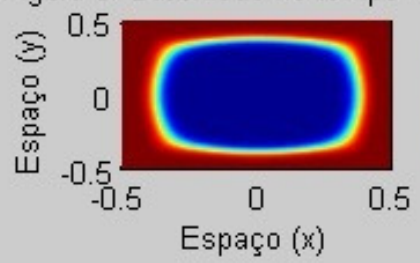

Figura b: Saturacao no tempo $=420$

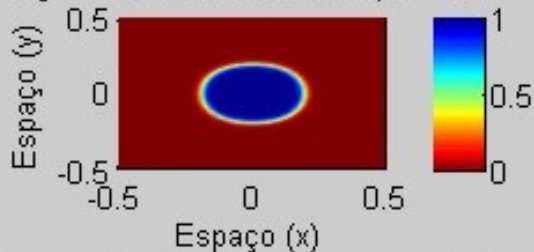

Figura d: Saturacao no tempo $=1260$

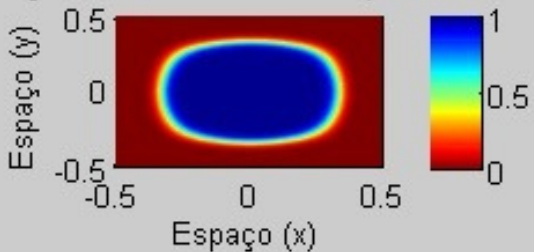

Figura f: Saturacao no tempo $=2100$

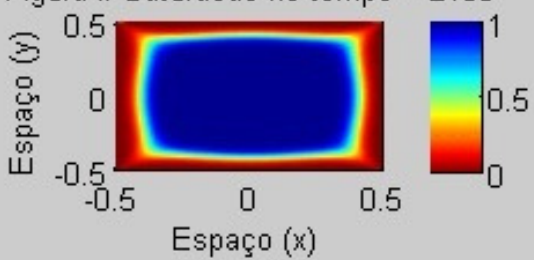

Figura 30: Problema de five spot. Resultado obtido usando o modelo da tese: Figura a: $t=t_{0}$,

Figura $b: t=t_{420}$, Figura $c: t=t_{840}$, Figura $d: t=t_{1260}$, Figura $:: t=t_{1680}$, Figuraf:

$$
t=t_{2100}
$$

A Figura 30 representa a solução numérica de saturação $U$ da água dada pela combinação das equações (3.11 e 6.10) e pela interpolação local por spline poliharmônica obtida na equação (3.17), em seis diferentes instantes de tempo, $t=t_{0}, t=t_{420}, t=t_{840}, t=t_{1260}, t=t_{1680}$ e $t=t_{2100}$. O código de cores à direita é semelhante ao da Figura 26 onde a cor vermelha representa o fluido puramente óleo, ou seja, saturação $U=O$ enquanto que a cor azul escuro indica o fluido puramente água, isto é, saturação $U=1$. Aqui também pode ser observado que a interface água/óleo está se deslocando do centro para os quatro poços das extremidades como apresentado anteriormente nos resultados gerados pelos autores Iske e Käser (2005).

Importante salientar que a intenção da pesquisa até aqui é apenas de validar o método Semi-Lagrangeano e a interpolação por spline poliharmônica, utilizando uma malha cartesiana regular. Portanto, o objetivo esperado até este ponto foi avaliar a propagação e o comportamento da injeção da saturação do fluido água se escoando para os quatros poços de produção. O objetivo é 
acrescentar adaptações ao programa desenvolvido com o intuito de evoluir e otimizar as aplicações propostas pelos autores Iske e Käser (2005) para o caso meshfree. Umas das melhorias é o cálculo do laplaciano dado no segundo membro da equação (3.5). Para este fim, esta pesquisa propôs utilizar os métodos meshfree de Hermite e de Kansa abordados no capitulo 4. A seguir serão apresentados alguns resultados e discussões baseando-se na combinação do método SemiLagrangeano, interpolação local por spline poliharmonica e os métodos meshfree.

\section{2. \\ Resultados do modelo adaptativo meshfree do problema 2D}

Nesta seção serão apresentados alguns resultados obtidos a partir do modelo desenvolvido dado pela combinação do método Semi-Lagrangeano, interpolação local por função base radial spline poliharmonica e os métodos meshfree de Hermite e de Kansa. Inicialmente na seção (6.2.1) será analisado e discutido os primeiros resultados, utilizando um domínio meshfree sem adaptação como estratégia para refinamento e remoção de nós. Em um segundo momento, na seção (6.2.2) será exibido o mesmo modelo sendo que agora utilizando as regras e estratégias de refinamento e remoção mencionados no capítulo 3. Somando-se as ilustrações, serão apresentadas observações e análises comparativas do método.

\subsection{1. \\ Resultados do modelo meshfree}

Esta seção apresenta os resultados encontrados, que tiveram por base primeiramente apenas a combinação do método Semi-Lagrangeano de pontos esparsos com os métodos meshfree, esta combinação se apresenta como solução para o problema de five spot. Além disso, fez-se uso da condição de contorno de Dirichlet para tratamento de fronteiras. Conforme já mencionado, o tratamento de fronteira é um dos maiores problemas dos métodos citados, por apresentarem pontos independentes sem conexão de fronteira e tendo em vista esta oportunidade de melhoria dada por esta deficiência no modelo de Dirichlet, esta tese trouxe como uma de suas contribuições, a adaptação no cálculo do laplaciano 
de pontos esparsos usando o método de Hermite e o método de Kansa como melhoria do método proposto pelos autores Iske e Käser (2005), que pode ser expandido para solução de qualquer tipo de EDP, segundo Greg Fasshauer (2007).

Para isso, primeiramente apresentam-se os resultados do cálculo de pressão dada pela mesma equação (6.12) e do campo de velocidade das fases para pontos esparsos, dada pela equação (6.11). Observa-se que na equação (6.12) o campo de pressão depende principalmente da distância entre pontos, pois a variação é apenas espacial.

Com a simplificação da lei de Darcy, isto é, o campo de velocidade da fase água ou da fase óleo é igual ao menos a gradiente de pressão e pode-se então dizer que o campo de velocidade das fases poderá ser obtido também pela simples derivada do campo de pressão em relação à direção $x$ e também em relação à direção $y$. Para um caso $2 D$, assumindo que um ponto do domínio tenha coordenadas $\left(x_{1}, x_{2}\right)$ a equação (6.11) pode ser rescrita como:

$$
\vec{V}=-\left(\frac{\partial P\left(x_{1}, x_{2}\right)}{\partial x_{1}}, \frac{\partial P\left(x_{1}, x_{2}\right)}{\partial x_{2}}\right)
$$

Onde o campo de velocidade de fase, na direção $x_{1}$ é dado por:

$$
\begin{aligned}
& \frac{\partial P\left(x_{1}, x_{2}\right)}{\partial x_{1}}= \\
& \sum_{c \in C}\left(2\left(x_{1}-c_{x_{1}}\right) \frac{1}{2}\left[\left(x_{1}-c_{x_{1}}\right)^{2}+\left(x_{2}-c_{x_{2}}\right)^{2}\right]^{\frac{1}{2}} \frac{1}{\ln (10) \sqrt{\left(x_{1}-c_{x_{1}}\right)^{2}+\left(x_{2}-c_{x_{2}}\right)^{2}}}\right) \\
& -\frac{x_{1}-o_{x_{1}}}{\sqrt{\left(x_{1}-o_{x_{1}}\right)^{2}+\left(x_{2}-o_{x_{2}}\right)^{2}}} \frac{1}{\ln (10) \sqrt{\left(x_{1}-o_{x_{1}}\right)^{2}+\left(x_{2}-o_{x_{2}}\right)^{2}}} \\
& =\frac{1}{\ln (10)}\left(\sum_{c \in C}\left(\frac{x_{1}-c_{x_{1}}}{\left[\left(x_{1}-c_{x_{1}}\right)^{2}+\left(x_{2}-c_{x_{2}}\right)^{2}\right]}\right)-\frac{x_{1}-o_{x_{1}}}{\left[\left(x_{1}-o_{x_{1}}\right)^{2}+\left(x_{2}-o_{x_{2}}\right)^{2}\right]}\right)
\end{aligned}
$$

E analogamente na direção $x_{2}$ é dada por: 


$$
\begin{aligned}
& \frac{\partial P\left(x_{1}, x_{2}\right)}{\partial x_{2}}= \\
& \left.\left.\frac{1}{\ln (10)}\left(\sum_{c \in C}\left(\frac{x_{2}-c_{x_{2}}}{\left[\left(x_{1}-c_{x_{1}}\right)^{2}+\left(x_{2}-c_{x_{2}}\right)^{2}\right.}\right]\right)-\frac{x_{2}-o_{x_{2}}}{\left(x_{1}-o_{x_{1}}\right)^{2}+\left(x_{2}-o_{x_{2}}\right)^{2}}\right]\right)
\end{aligned}
$$

Além disso, o campo de velocidade de advecção poderá ser dado pela variação da função fluxo fracional $f(U)$ (equação 2.15), com relação à saturação $U$, do passo anterior. Então, na forma de expressão tem-se que:

$$
\vec{V}=\frac{\partial f(U)}{\partial U}=\frac{2 U \mu(1-U)}{\left[U^{2}-\mu(1-U)^{2}\right]^{2}}
$$

Nesta pesquisa, o domínio foi representado por um conjunto de pontos esparsos conhecido na comunidade científica como Halton points (Halton, 1960; Wong et al, 1997), para aplicações multidimensionais. O número de nós no domínio, Halton sugere como,

$$
N_{-} \text {epslon }=\left\{\left(2^{k}+1\right)^{2}\right\}=\{9,25,81,289,1089,4225,16641, \ldots\}
$$

Utilizando as mesmas condições iniciais, como o raio de injeção e a posição do poço de injeção e dos poços de produção, dadas anteriormente, o campo de pressão e o campo de velocidade de dados esparsos são exibidos nas figuras abaixo que se seguem: 


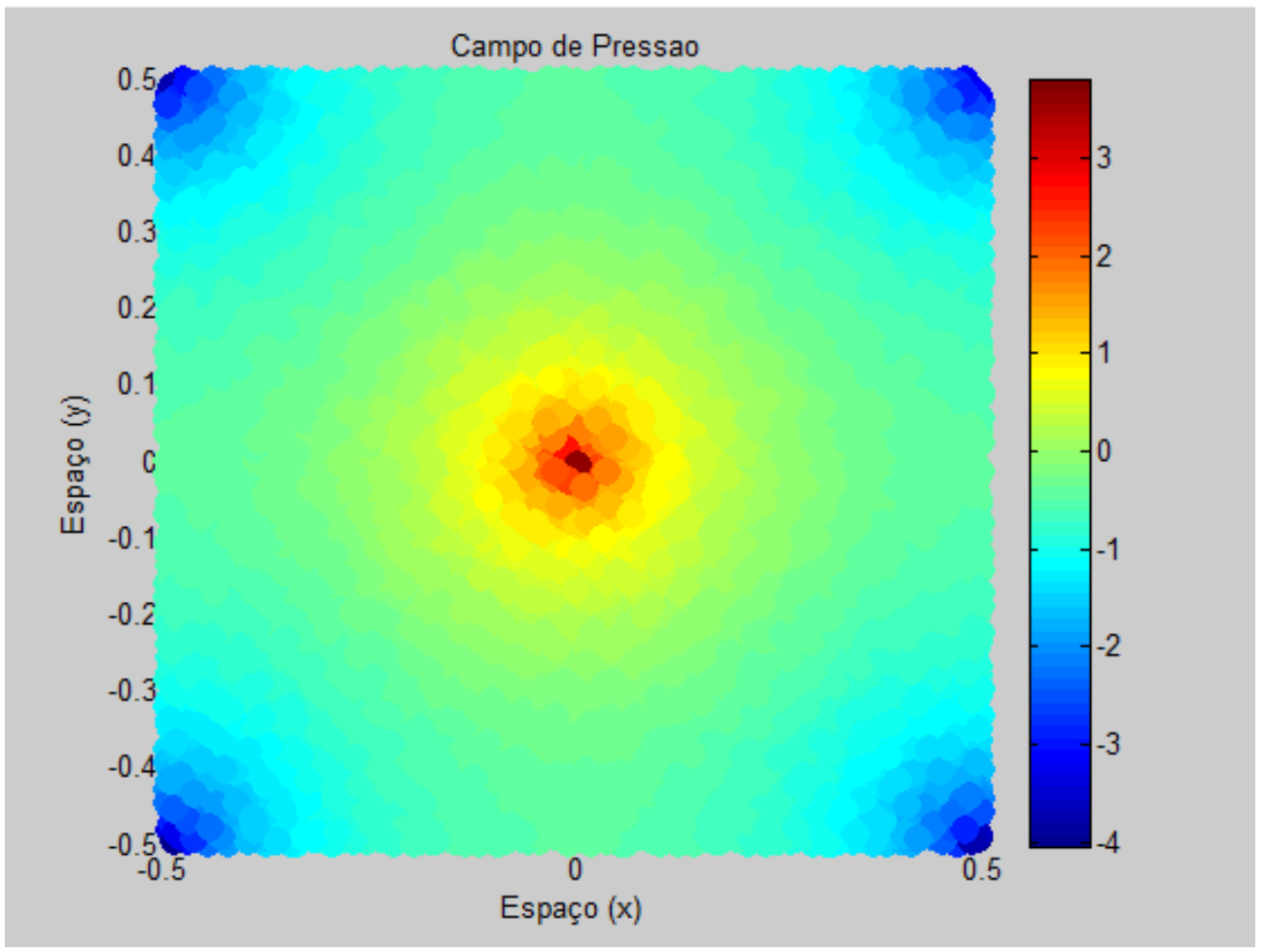

Figura 31: Campo de pressão do problema five spot. Resultado obtido usando o modelo SemiLagrangeano meshfree

Como sugerido na modelagem a Figura 31 ilustra o campo de pressão do problema de five spot, obtido usando o modelo Semi-Lagrangeano adaptativo. Enquanto que a Figura 32 exibe o campo de velocidade também do modelo SemiLagrangeano adaptativo correspondente ao mesmo problema. 


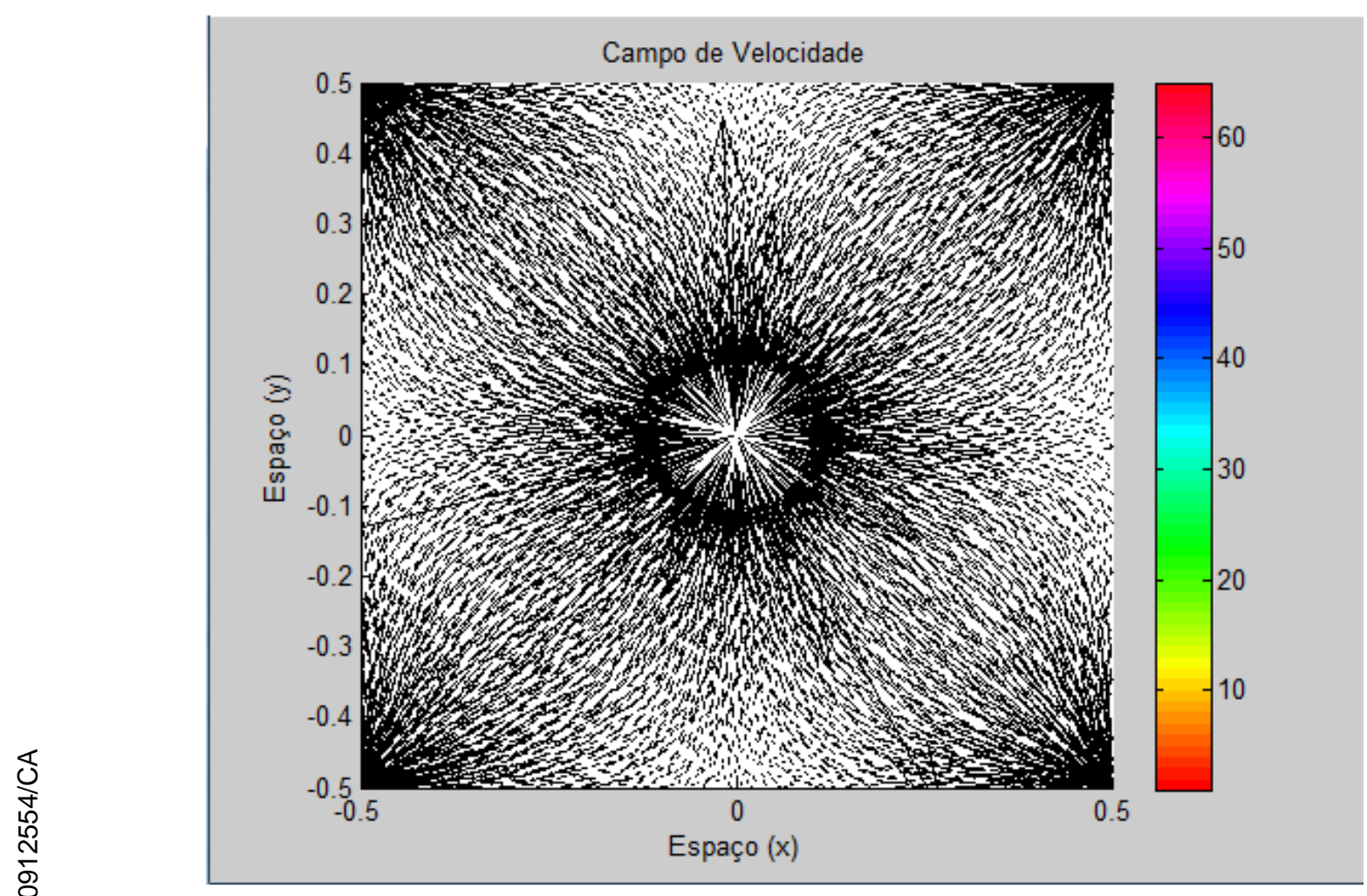

Figura 32: Campo de velocidade do problema five spot. Resultado obtido usando o modelo SemiLagrangeano meshfree

Como pode se notar o campo de pressão e o campo de velocidade tem o mesmo comportamento dado em relação ao campo de pressão e o campo de velocidade gerado na malha regular cartesiana. Portanto, mostrando assim o fenômeno da variação do campo de pressão e do campo de velocidade com a injeção de água no centro do domínio se propagando até os quatro poços de produção.

Apresentam-se a seguir, os resultados obtidos do campo de saturação, neste caso usando a combinação do modelo Semi-Lagrangeano com os métodos meshfree para pontos esparsos em diversos instantes de tempo, variando assim o número de pontos. A escolha do número de pontos será feita de acordo com a formação proposta por Halton (equação 6.17). Sendo assim, usando as mesmas condições iniciais propostas pelos autores e aplicadas juntamente com o algoritmo proposto no capitulo 3, determina-se o upstream point baseado nas equações $(3.12 ; 3.14 ; 3.15)$.

Como já sabido, a intenção neste dado momento é encontrar a saturação ideal do upstream point pela interpolação, para isso, advectando o mesmo ao 
longo do tempo. A principal diferença em relação aos resultados obtidos anteriores e os que são apresentados a seguir é que em vez de uma malha com espaçamento regular, tem-se agora um domínio composto por um conjunto de pontos esparsos. Além disso, todas as funções foram generalizadas para serem usadas em qualquer situação, isto é, independentemente de se ter uma malha ou não, desde o cálculo dos vizinhos até a advecção.

Então, após se ter determinado o upstream point determinam-se os vizinhos do mesmo ponto para calcular a saturação. A função para o cálculo dos vizinhos se baseia na distância mínima entre os pontos. $\mathrm{O}$ valor da distância mínima utilizada inicialmente depende principalmente do número de pontos, pois, a ideia é ter um número de vizinhos controlados para uma boa aproximação do upstream point. Após o cálculo dos vizinhos também foi formulada uma nova função, mais robusta para interpolação do upstream points, usando aqui a função base por spline poliharmônica esparsa, agregado ao cálculo do laplaciano para dados esparsos. Para o cálculo do laplaciano utilizou-se os métodos meshfree e após a obtenção da saturação e do laplaciano do upstream point determinou-se a advecção do mesmo ponto dada pela equação (3.11).

Para determinar a advecção do novo campo da saturação é necessário também obter o laplaciano do upstream point pela interpolação por spline polihâmonica no segundo membro da equação (3.11). Mas, a interpolação local depende do campo do laplaciano do instante anterior. Então, neste caso, para determinar o campo do laplaciano utilizou-se os métodos meshfree, sendo eles, o método de Hermite e o método de Kansa, já citados. Note que a equação que descreve o fenômeno físico-matemático do laplaciano é uma equação diferencial elíptica parcial, como mostra a equação (4.5).

Segundo Gregorry Fasshauer (2007), uma das melhores formas de calcular o laplaciano de pontos esparsos é usar a estratégia de tratamento de fronteira, usando condições de contorno. Sendo assim, neste caso, utilizou-se a condição de contorno proposta por Dirichlet mencionada na equação (4.6). Essa estratégia foi feita colocando pontos na fronteira, conforme ilustra a Figura 33. Esta modelagem pode ser feita de forma local ou de forma global, ou seja, a interpolação ou o laplaciano do ponto requerido pode ser feita de enumeras formas, dependo do problema a ser resolvido. 


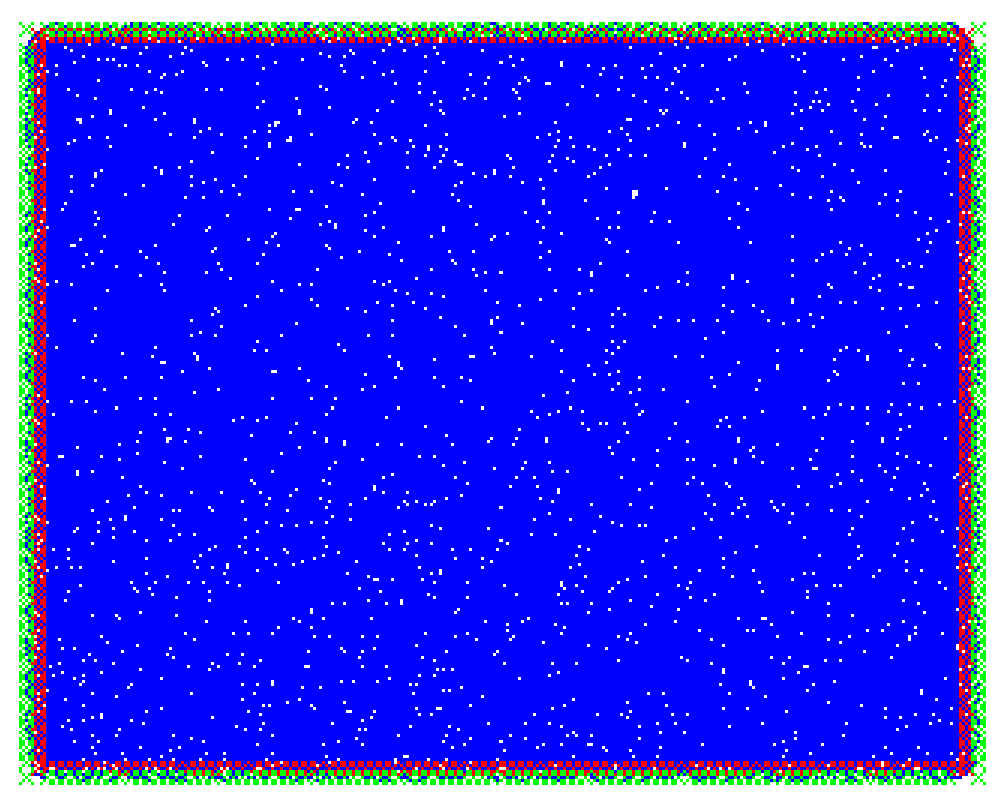

Figura 33: Forma de colocação de pontos na fronteira

Observe que essa estratégia envolve novos pontos no domínio, isso implica a necessidade de obter a saturação destes novos pontos para resolver o campo do laplaciano. Utilizado a modelagem do método de Hermite ou do método de $K a n s a$, é possível ter uma função que representa uma aproximação da saturação em qualquer ponto do domínio. Nos problemas propostos por Gregory Fasshauer sempre havia uma função dada pelo problema. Esta pesquisa adotou como premissa a ideia de construir uma função para cada instante de tempo que representasse a aproximação do campo da saturação do instante de tempo anterior, usando a condição de fronteira de Dirichlet.

As figuras abaixo foram simuladas com as seguintes informações:

- Número de pontos, $N \_$epslon $=4225$;

- Distância mínima entre os pontos, Dist_Min =0.06;

- As outras informações usadas foram as mesmas apresentadas nos exemplos anteriores.

Da Figura 34 à Figura 37 são exibidas os principais resultados do campo da saturação $U$ em diferentes instantes de tempo do modelo Semi-Lagrangeano combinado com os métodos meshfree para advecção do problema de injeção de água de five spot. Essas figuras foram geradas inicialmente usando o método 
simétrico de Hermite de acordo com a formulação feita na seção (4.1.2) e mais a frente apresentam-se os mesmos resultados, sendo que neste segundo momento utilizando o método assimétrico de Kansa de acordo com a formulação dada na seção (4.1.1). Além disso, utilizou-se como mencionado anteriormente, a interpolação local por spline poliharmônica para todos os casos. Lembrando que a proposta inicial veio da solução da equação de advecção de Buckley-Leverett viscoso para problemas de reservatório de petróleo.

Relembrado o código de cores ilustrado. À direita das figuras, a cor vermelha representa o fluido puramente óleo, ou seja, saturação $U=0$ enquanto que a cor azul escura indica o fluido puramente água, isto é, saturação $U=1$. A combinação das cores entre vermelha e azul escuro mostra a variação da saturação $U$ da água, ao longo do tempo. Note que a interface água / óleo está se deslocando do centro do domínio para os quatro poços das extremidades.

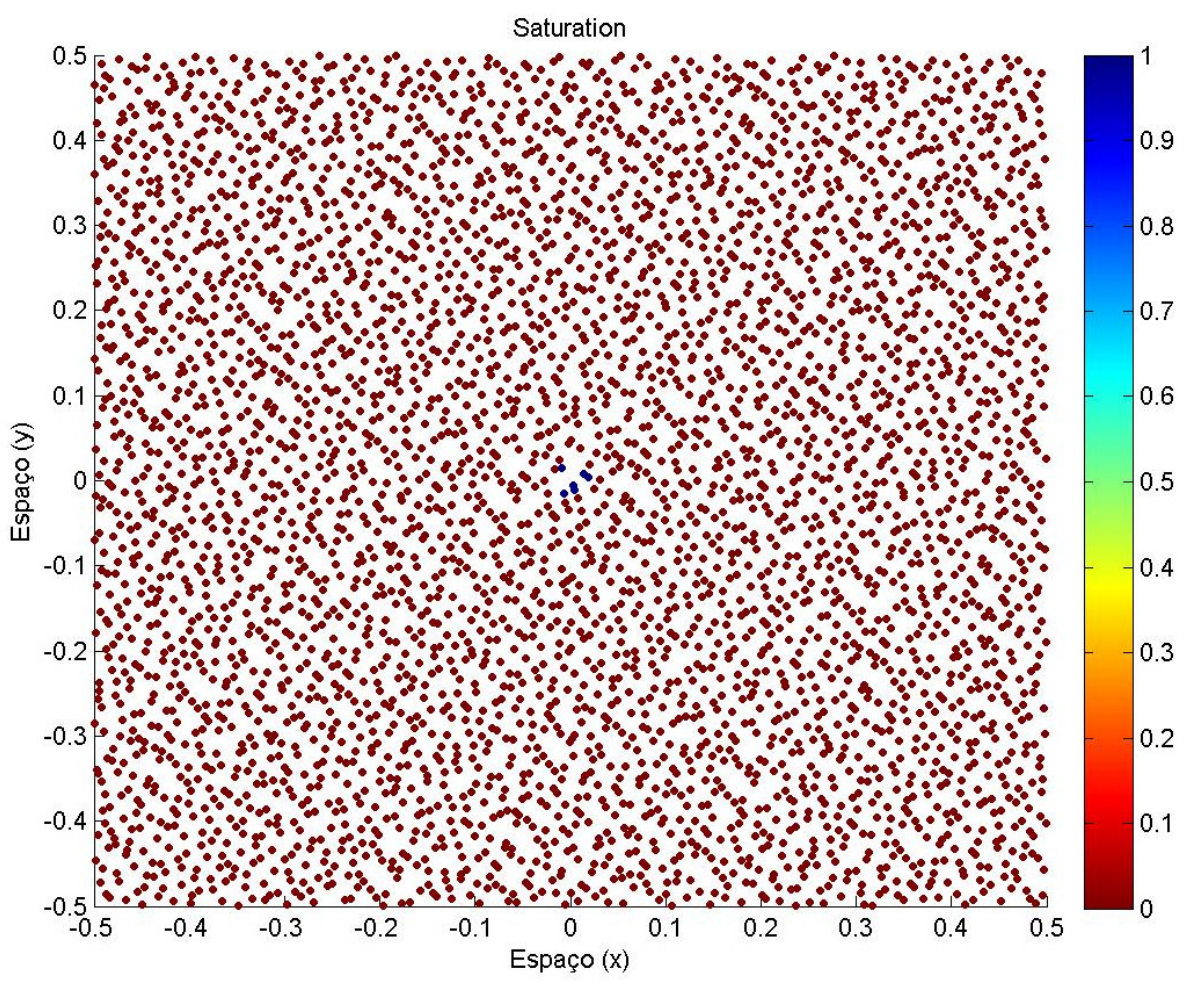

Figura 34: Saturação do modelo adaptativo do Problema five spot

$$
t=1
$$

A Figura 34 apresenta a condição inicial do campo da saturação proposto pelos autores Iske e Kaser (2005), no primeiro instante de tempo. 


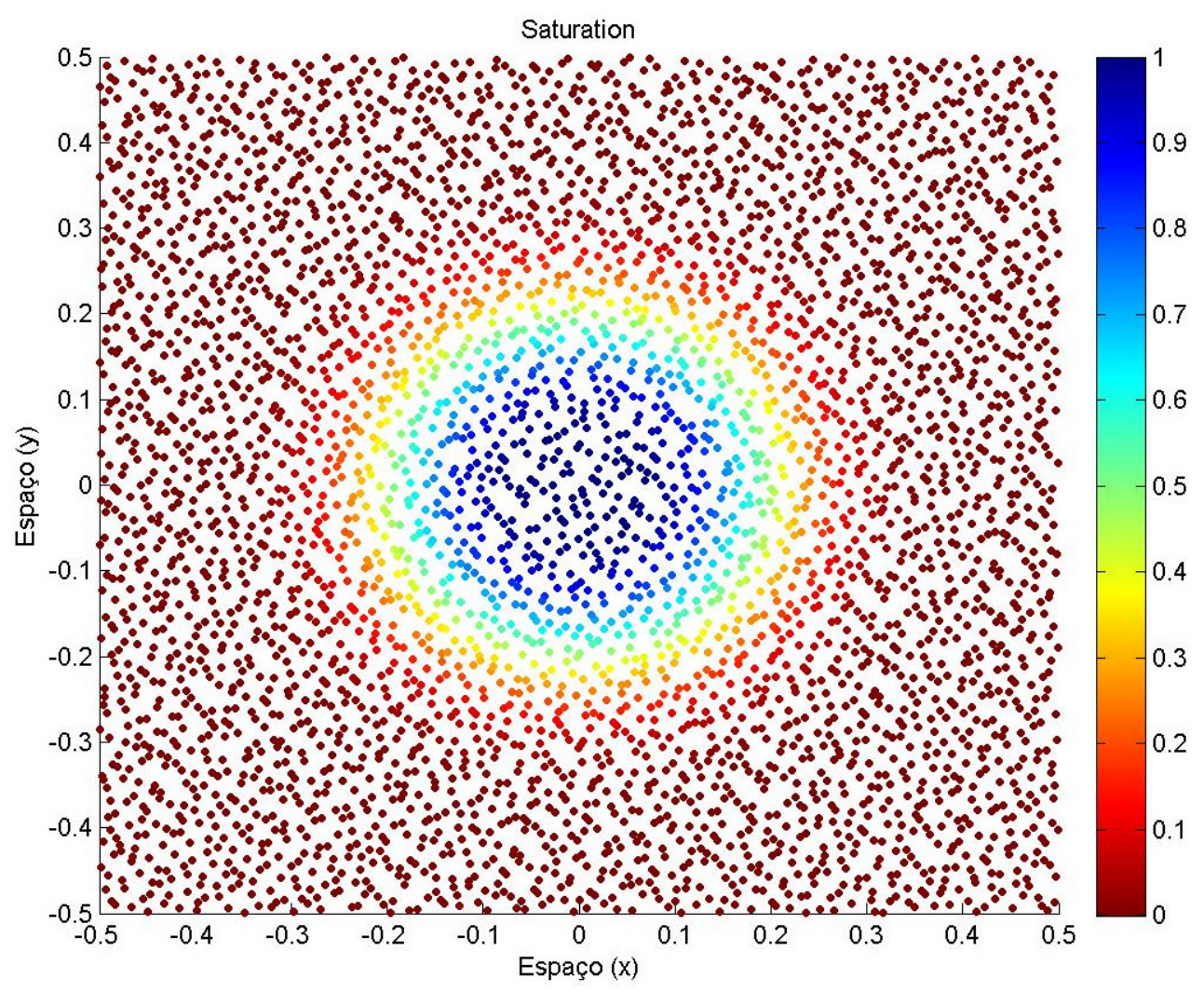

Figura 35: Saturação do modelo adaptativo do problema five spot utilizando o método de Hermite

$$
t=420
$$

A Figura 35 sinaliza a saturação no instante de tempo igual a 420. Como pode ser observado na figura há uma variação do campo de saturação de forma simétrica e harmônica, isto representa o comportamento e variação da saturação de um reservatório homogênea, ou seja, a permeabilidade absoluta e a porosidade do meio são constantes. 


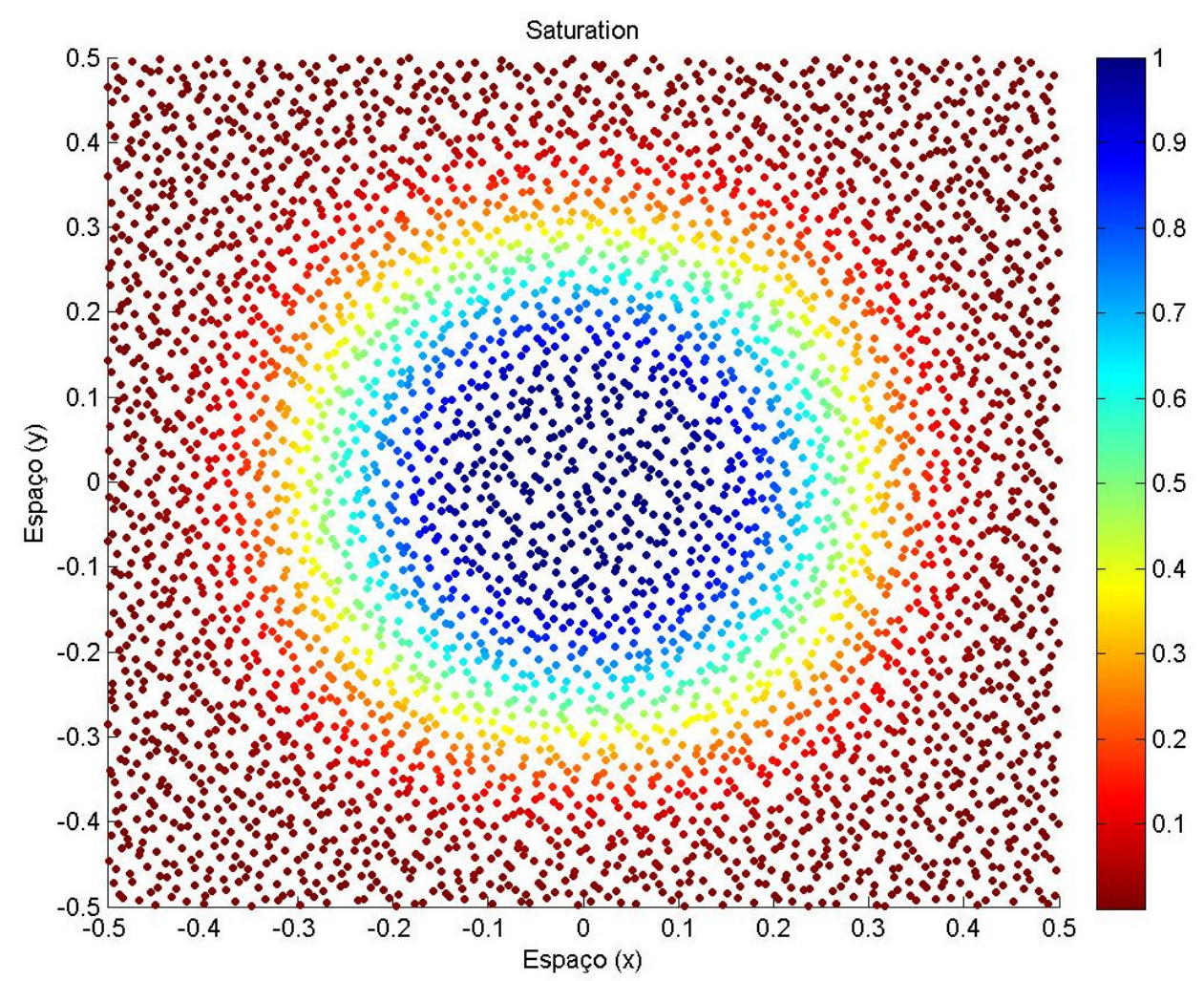

Figura 36: Saturação do modelo adaptativo do problema five spot utilizando o método de Hermite

$$
t=840
$$

As ilustrações da Figura 36 à Figura 39 demostram o campo de saturação $U$ nos instantes de tempo 840, 1260, 1680 e 2100 respectivamente. Portanto, essas figuras representam a injeção do fluido água do centro do reservatório através do raio da injeção e o fluido óleo sendo empurrado pela saturação da água ao longo do tempo, para os quartos outros poços de produção como esperado. 


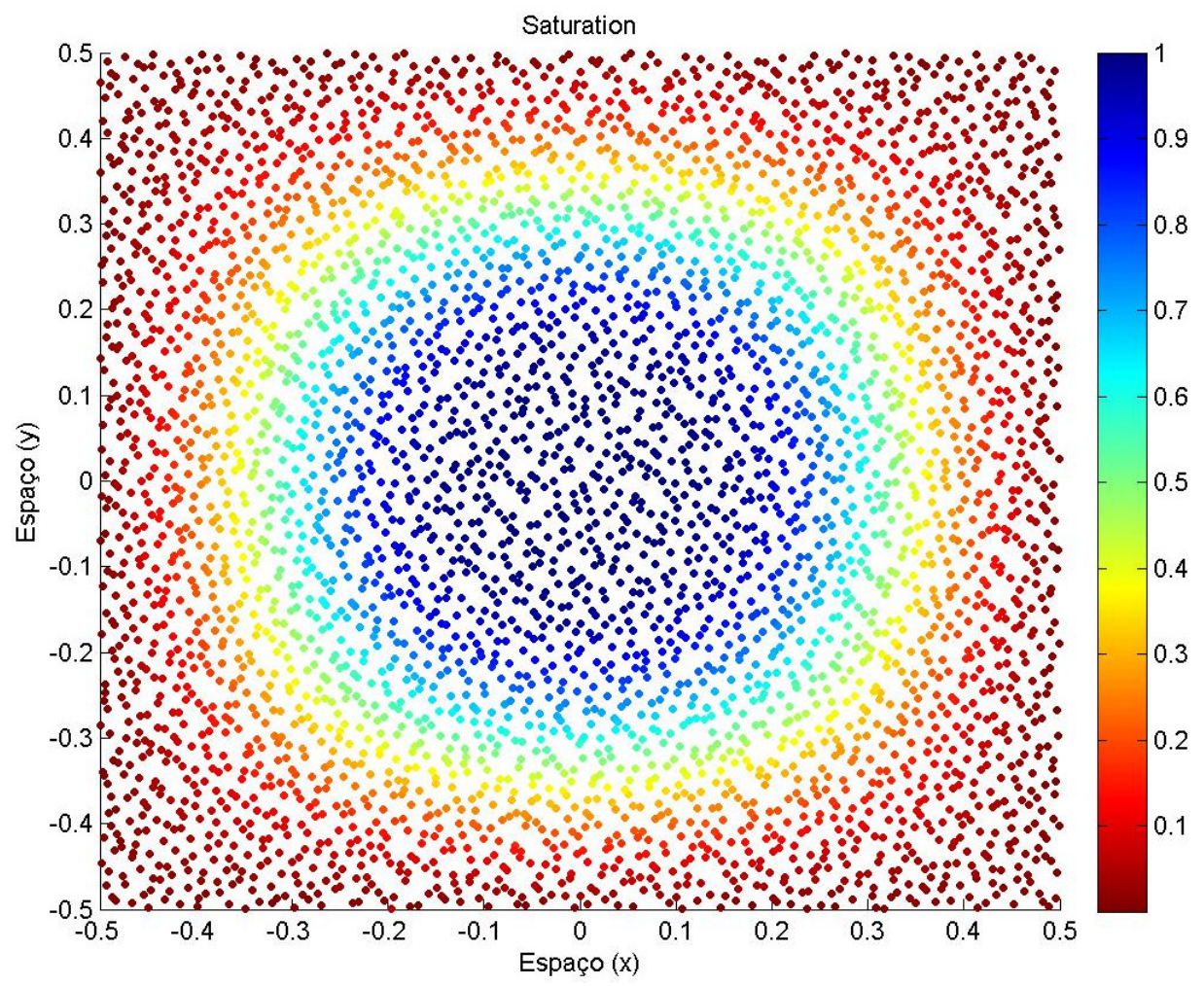

Figura 37: Saturação do modelo adaptativo do problema five spot, utilizando o método de Hermite

$t=1260$

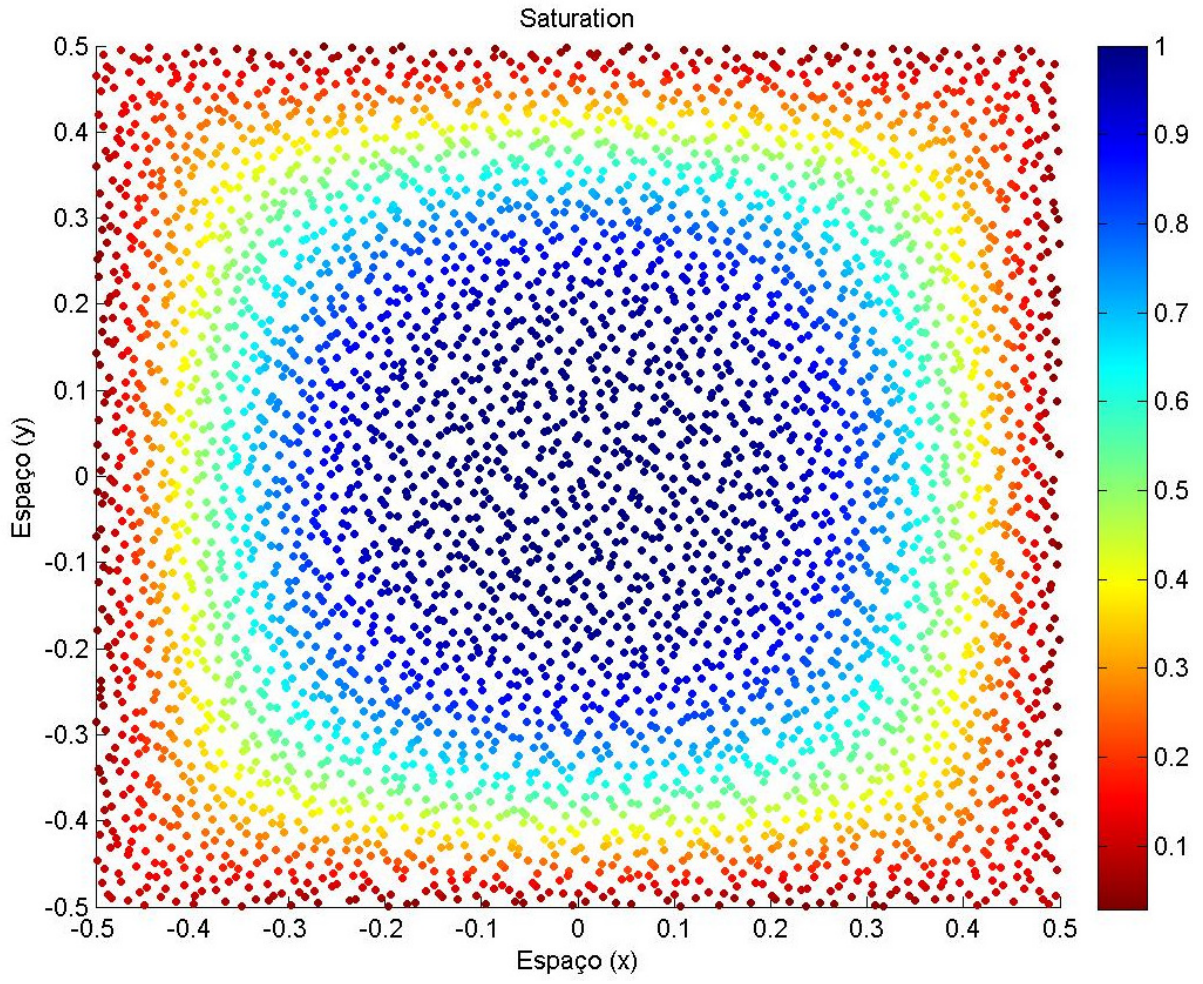

Figura 38: Saturação do modelo adaptativo do problema five spot, utilizando o método de Hermite

$t=1680$ 


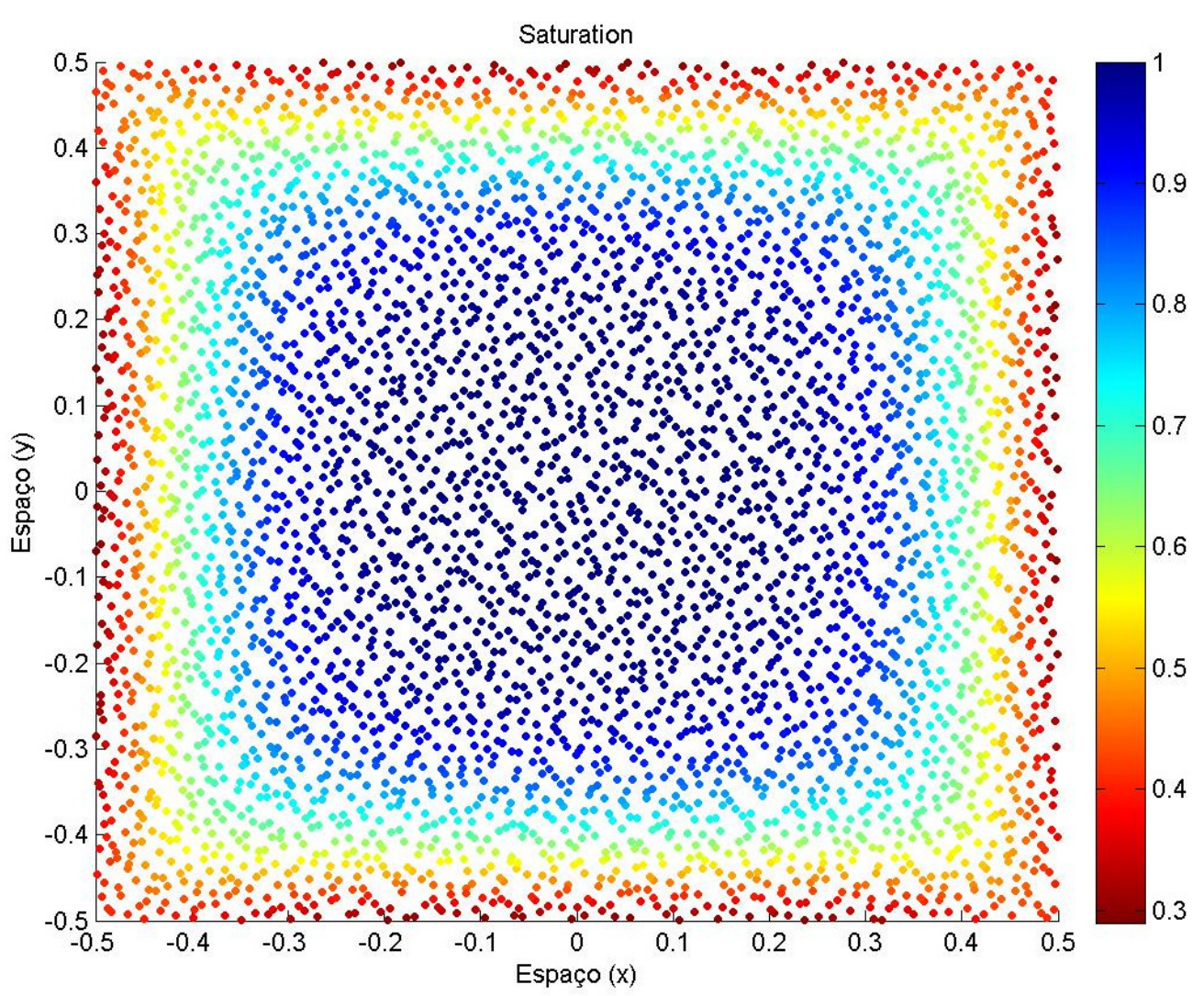

Figura 39: Saturação do modelo adaptativo do problema five spot, utilizando o método de Hermite

$$
t=2100
$$

As figuras a seguir ilustram também o campo de saturação usando agora o modelo Semi-Lagrangeano em combinação com o método de Kansa, utilizando a mesma condição de fronteira de Dirichlet. Logo, da Figura 40 à Figura 43 são ilustrados os campos de saturação nos instantes de tempo 840, 1260, 1680 e 2100, respectivamente. Como se pode constatar também que há uma variação da saturação ao longo do tempo. É importante salientar ainda que a cor azul prevalece em grande quantidade significando fisicamente que uma injeção contínua do fluido, água, está empurrando em grande proporção o fluido, óleo, para os quatro poços de produção por meio de sobrepressão, efeito conhecido na literatura como produção primaria (Peaceman, 1977). Em determinado instante de tempo, a pressão chega a um estado de equilíbrio e o óleo deixa de fluir, sendo necessária uma nova pressão mais elevada no reservatório, de modo que a produção do óleo continue. Este fenômeno, também conhecido na literatura como 
uma produção secundária e conforme apresentado no capítulo 5 pode ser tratado no novo modelo proposto por esta pesquisa. Um exemplo de produção secundaria será ilustrado na seção 6.3 do novo modelo proposto nesta pesquisa, em combinação com os métodos utilizando um meio poroso heterogêneo.

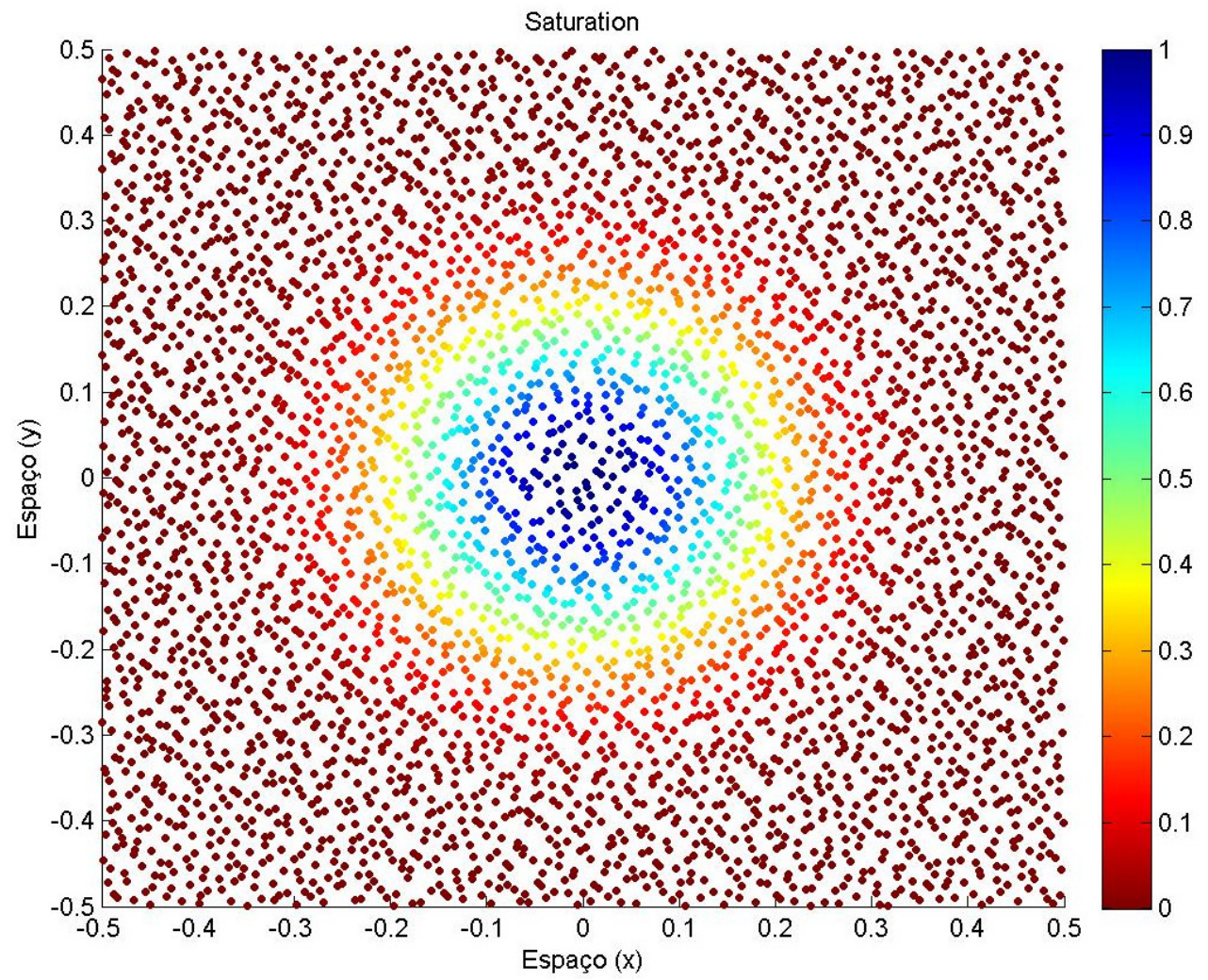

Figura 40: Saturação do modelo adaptativo do problema five spot, utilizando o método de Kansa

$$
t=840
$$




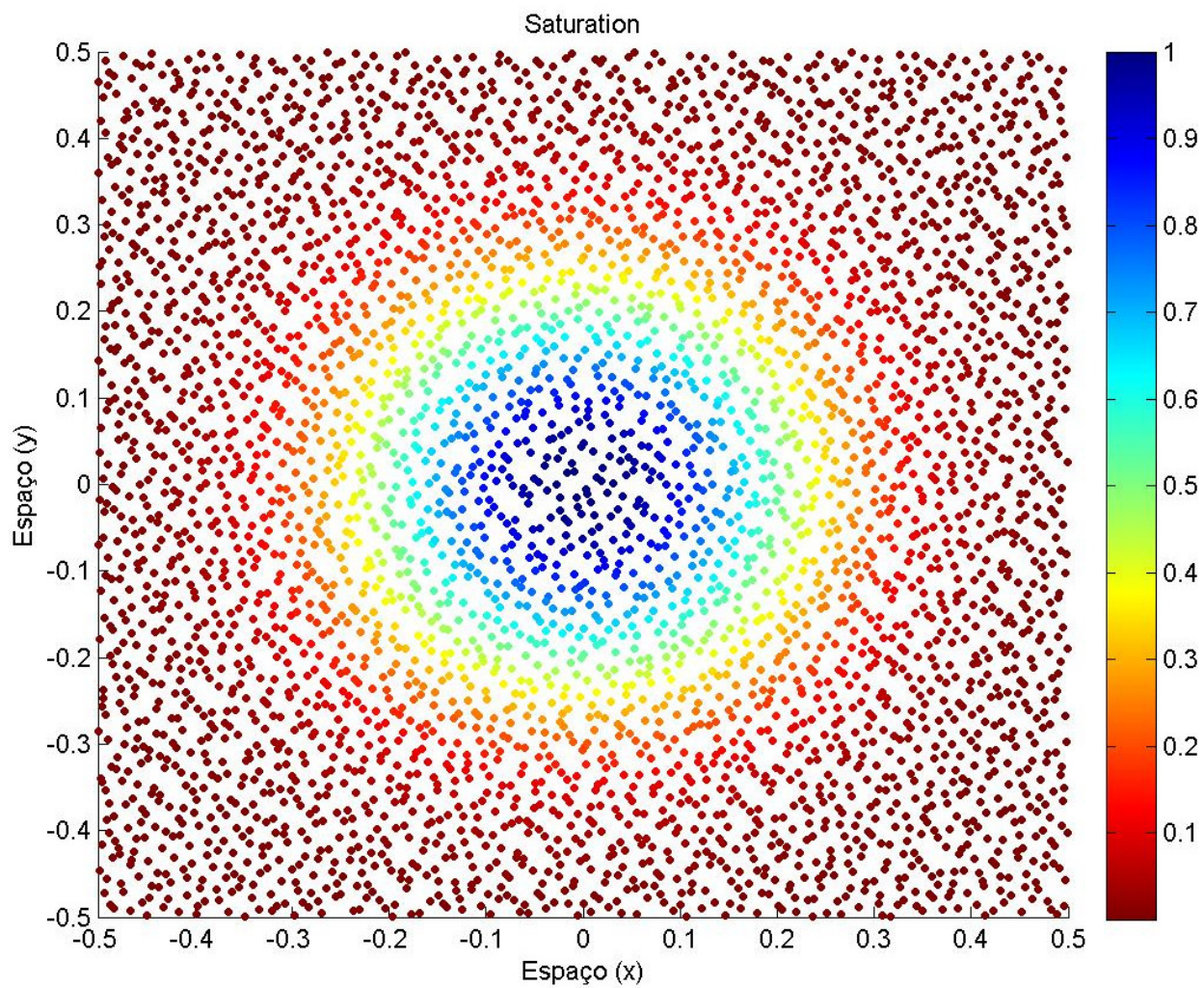

Figura 41: Saturação do modelo adaptativo do problema five spot, utilizando o método de Kansa

$$
t=1260
$$

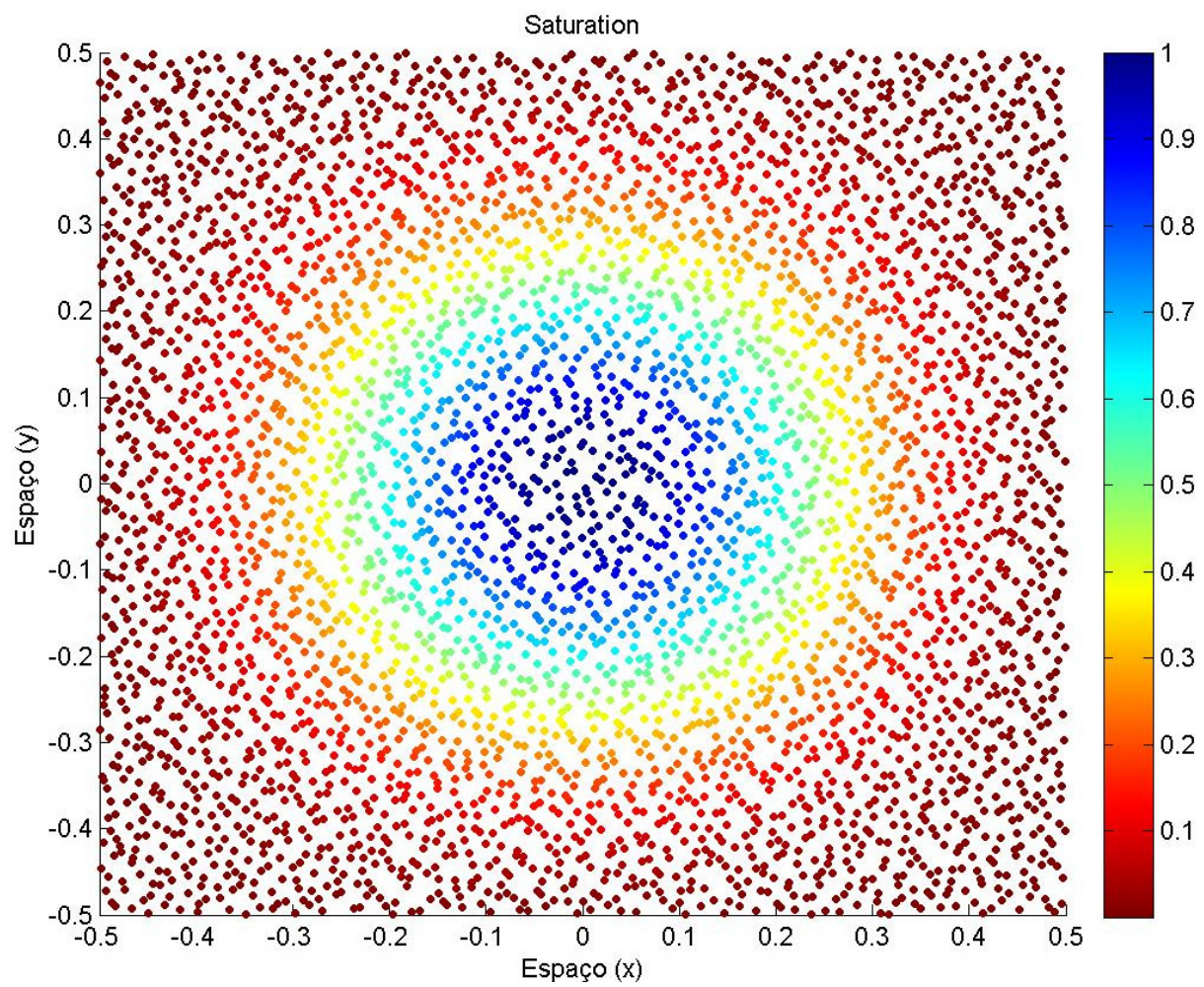

Figura 42: Saturação do modelo adaptativo do problema five spot, utilizando o método de Kansa

$$
t=1680
$$




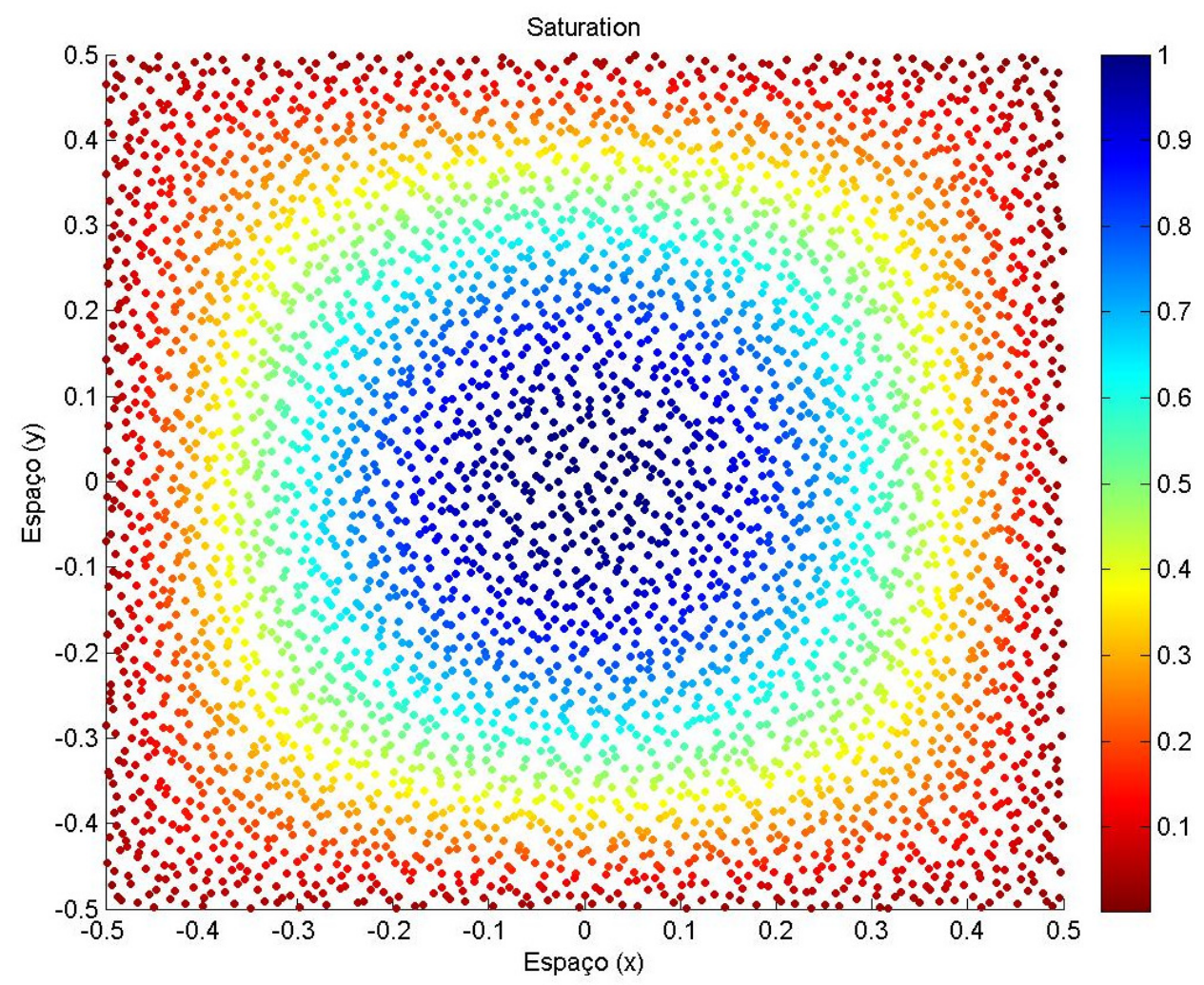

Figura 43: Saturação do modelo adaptativo do problema five spot, utilizando o método de Kansa

$$
t=2100
$$

Uma análise relevante sobre as matrizes propostas por Hermite e por Kansa é que a colocação dos pontos na fronteira influenciará de forma crucial na criação da matriz determinando se será simétrica ou antissimétrica. Além disso, a colocação implicará também na estabilidade e na convergência do modelo sugerido. Para generalizar o modelo proposto, dependendo do caso, pequenas relações devem ser feitas durante a simulação para haver uma boa interação entre a geometria, o raio de injeção e a distância mínima. O número de pontos colocados na vizinhança e a escolha da função de base radial, bem como, os tratamentos de singularidades que eventualmente podem surgir durante o processo de simulação, são fundamentais para uma boa convergência da modelagem.

A seguir, serão apresentados testes do modelo de refinamento e remoção de nós. 


\subsection{2. Resultados do Modelo Adaptativo}

Uma das melhores formas de avaliar e analisar o processo de refinamento e remoção de nós em um modelo é baseando-se e considerando um domínio muito inferior ao domínio proposto inicialmente. Para este fim, esta seção consiste em ilustrar e discutir dois exemplos de processo de refinamento e remoção de nós empregando as estratégias mencionadas no algoritmo da seção (3.2).

O processo de refinamento e remoção de nós consiste na melhoria da qualidade do modelo e não na otimização do modelo. Primeiramente é apresentado um exemplo do modelo de refinamento e remoção considerando uma malha cartesiana regular. A primeira avaliação se baseia em um teste feito propositalmente para que haja um grande número de pontos com refinamento, analisando ao mesmo tempo a convergência do modelo. Como a geometria é regular então o exemplo é feito utilizando o modelo de midpoint para o refinamento ou inserção dos nós.

A segunda avaliação se baseia no modelo de meshfree utilizando um domínio totalmente disperso e com pequenas falhas na forma como que estão dispostos os nós. A ideia proposta foi a utilização do algoritmo de refinamento e remoção do modelo meshfree para obter a melhor correção possível ao longo do tempo. Por ser uma geometria meshfree o refinamento é dado pela inserção de pontos baseado no modelo de diagrama de Voronoi, Preparata e Shamos, (1985) e Devadoss e Rourke (2011). O diagrama de Voronoi, também conhecido como tesselação de Dirichlet é uma estrutura geométrica formada por polígonos convexos regulares ou não, obtidos a partir de um conjunto arbitrário de pontos no espaço euclidiano. É uma tesselação que permite determinar, no espaço Multidimensional, a região de domínio dos pontos dados e os vizinhos mais próximos. Sendo assim, é possível determinar com base numa triangularização de pontos, a área de influencia de cada um desses pontos em função da posição do outros pontos, delimitando e minimizando geometricamente cada área de influência. Deste modo, dado um conjunto de nós inicial $\Xi \subset \mathfrak{R}^{d}$, considerando que qualquer nó $\xi \in \Xi$, a grelha de Voronoi é dada da seguinte forma: 


$$
\text { Voronoi }_{\Xi}(\xi)=\left\{x \in \mathfrak{R}^{d} \quad: \operatorname{dist}_{\Xi}(x)=\|x-\xi\|\right\} \subset \mathfrak{R}^{d}
$$

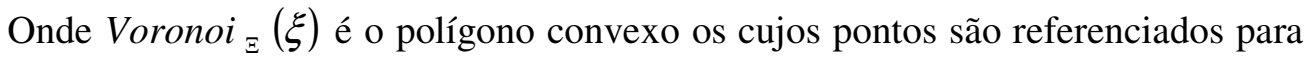
serem os pontos de Voronoi, formando um conjunto finito de pontos na vizinhança de $\xi$.

Da Figura 44 à Figura 47 ilustram o processo de refinamento e remoção do modelo usando o método de Hermite em combinação com o modelo adaptativo nos instante de tempo 1, 5, 109 e 216, respectivamente. O domínio é composto por 96 nós, sendo que 64 pontos são considerados como os pontos interiores e 8 pontos em cada uma das 4 fronteiras, totalizando 96 pontos $=64+8+8+8+8$.

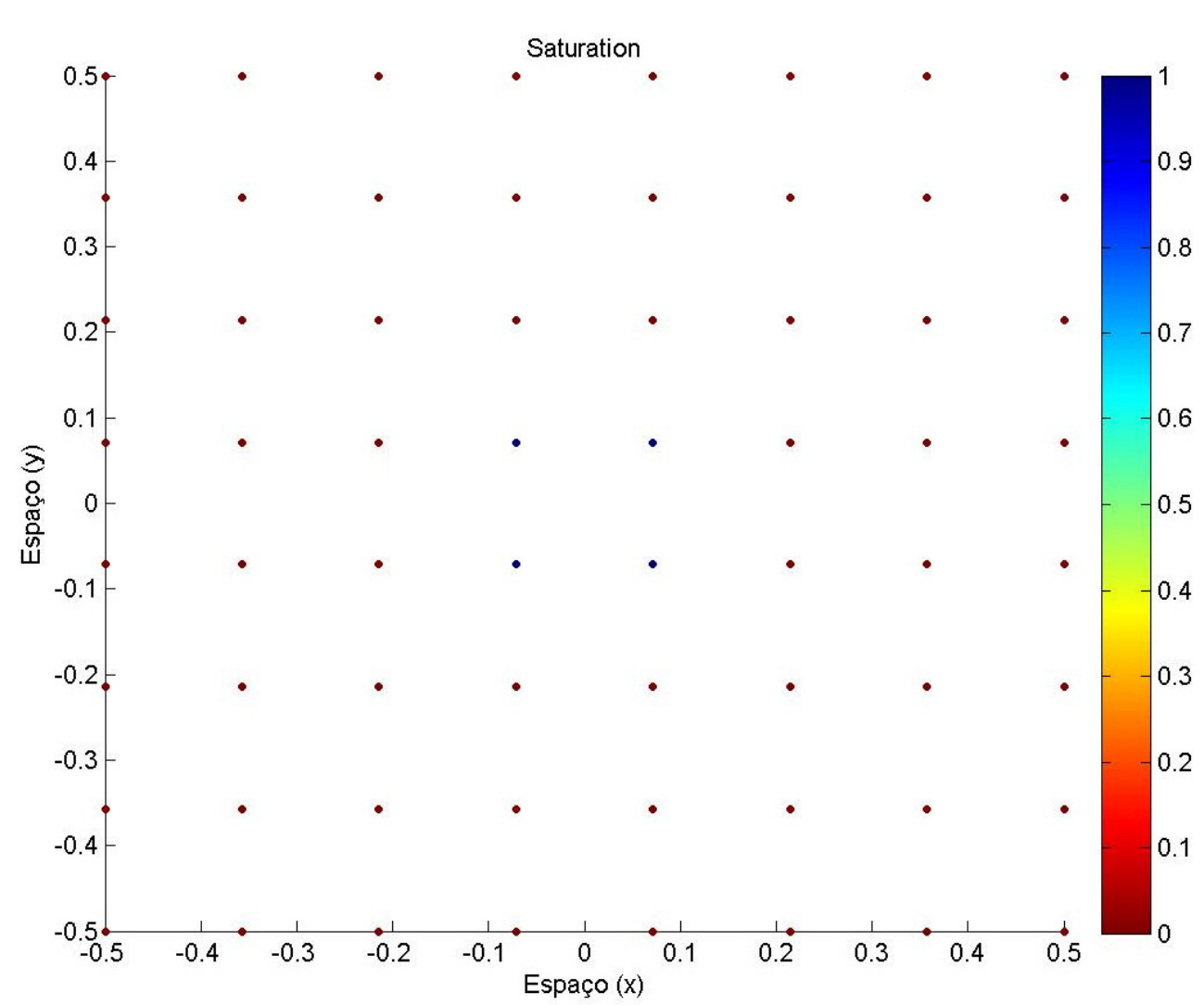

Figura 44: Saturação do modelo adaptativo do problema five spot, utilizando uma malha regular

$$
t=1
$$




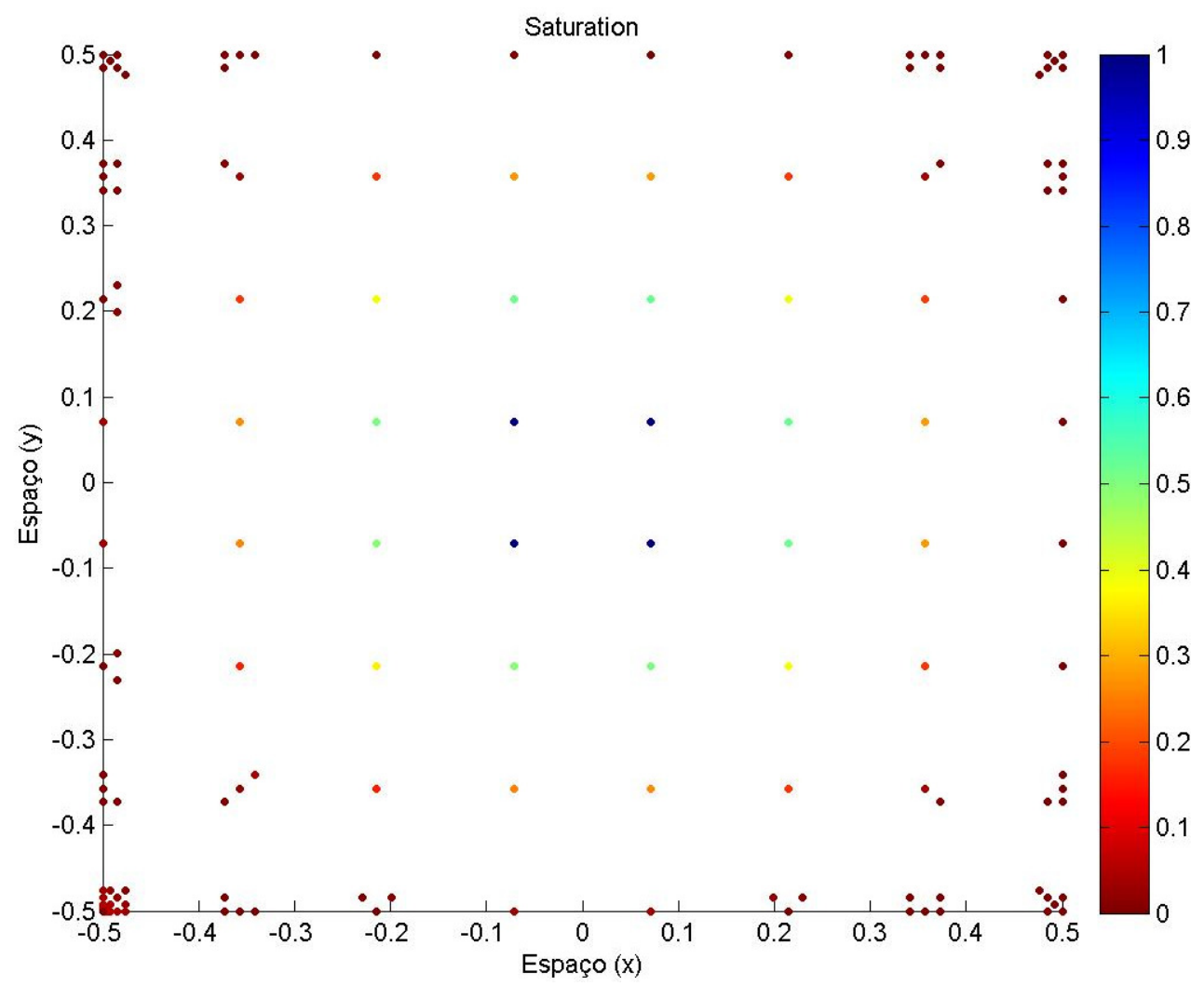

Figura 45: Saturação do modelo adaptativo do problema five spot, utilizando uma malha regular

$$
t=5
$$

Como os pontos não são processados ao mesmo tempo, isto é, pontos simétricos geometricamente deixam de ser simetricos com a relaçao ao valor de saturaçao. 


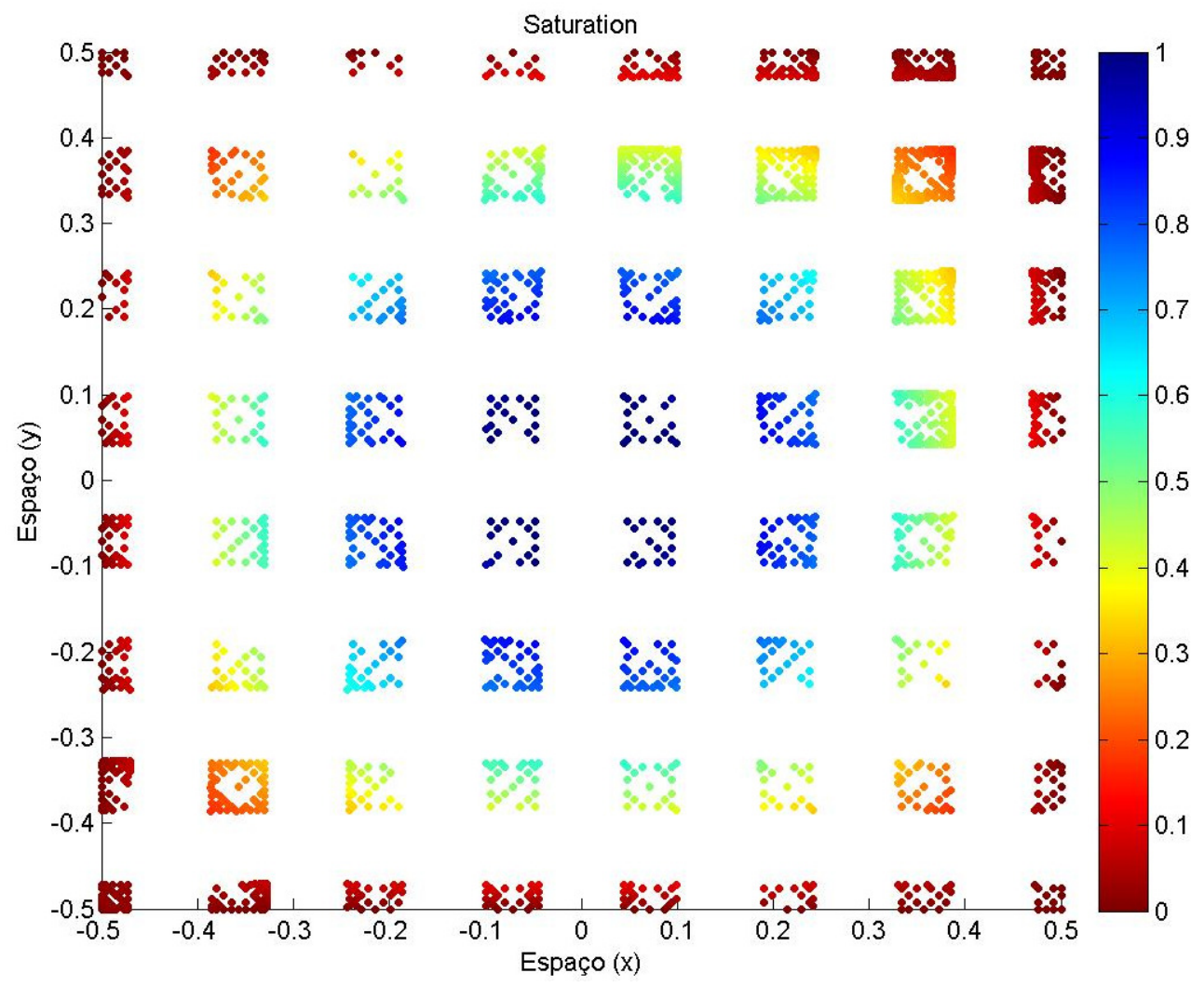

Figura 46: Saturação do modelo adaptativo do problema five spot, utilizando uma malha regular

$$
t=109
$$




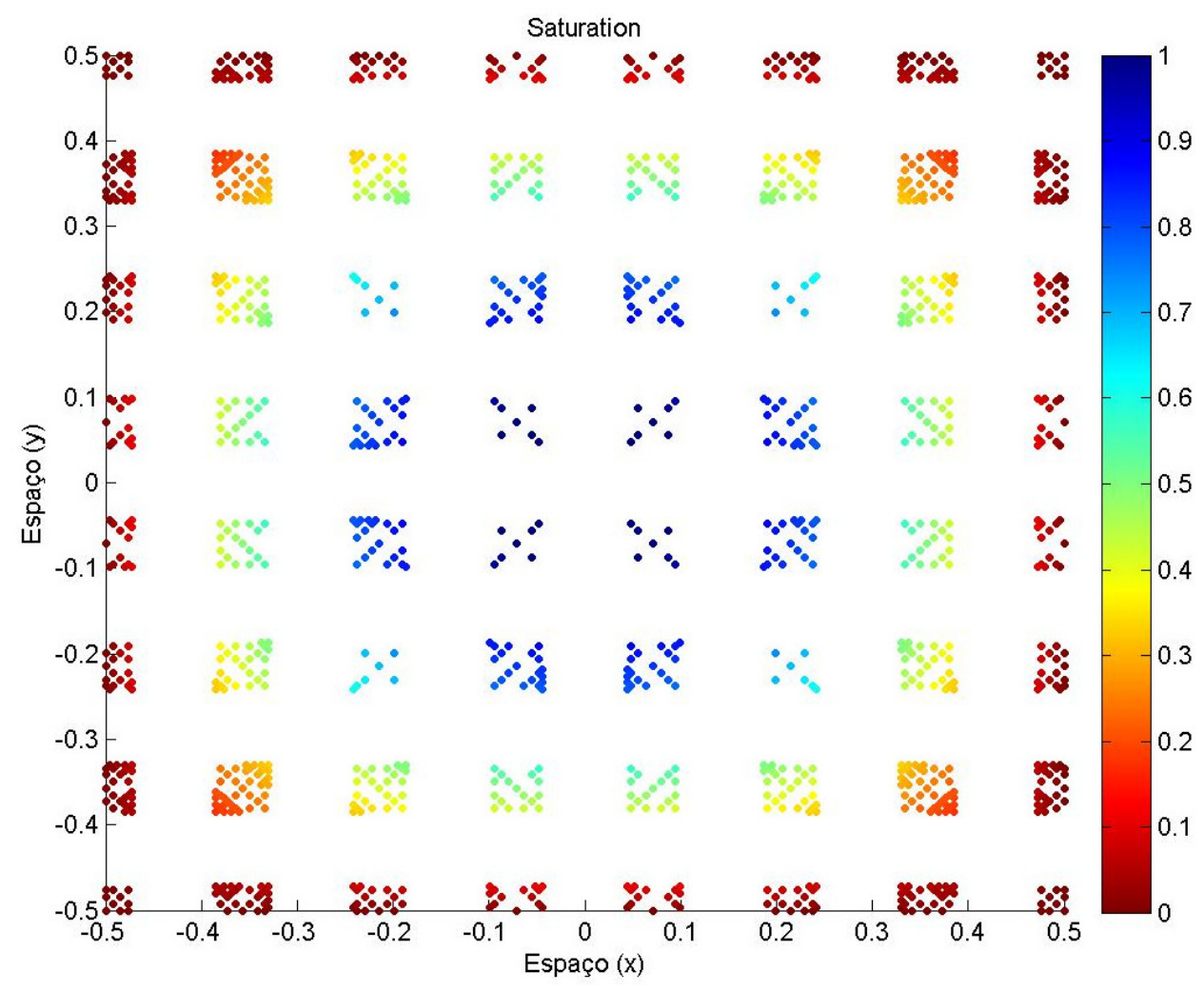

Figura 47: Saturação do modelo adaptativo do problema five spot, utilizando uma malha regular

$$
t=216
$$

O segundo exemplo é baseado na inserção por pontos de Voronoi. O principal objetivo deste exemplo é ilustrar o processo de refinamento através da remoção e inserção de pontos. Da Figura 48 à Figura 51, exibem o processo de refinamento e remoção, utilizando a estratégia de inserção por pontos de Voronoi nos instantes de tempo de 1, 8, 75 e 179, respectivamente. As figuras supracitadas da combinação do método de Hermite com o modelo adaptativo. 


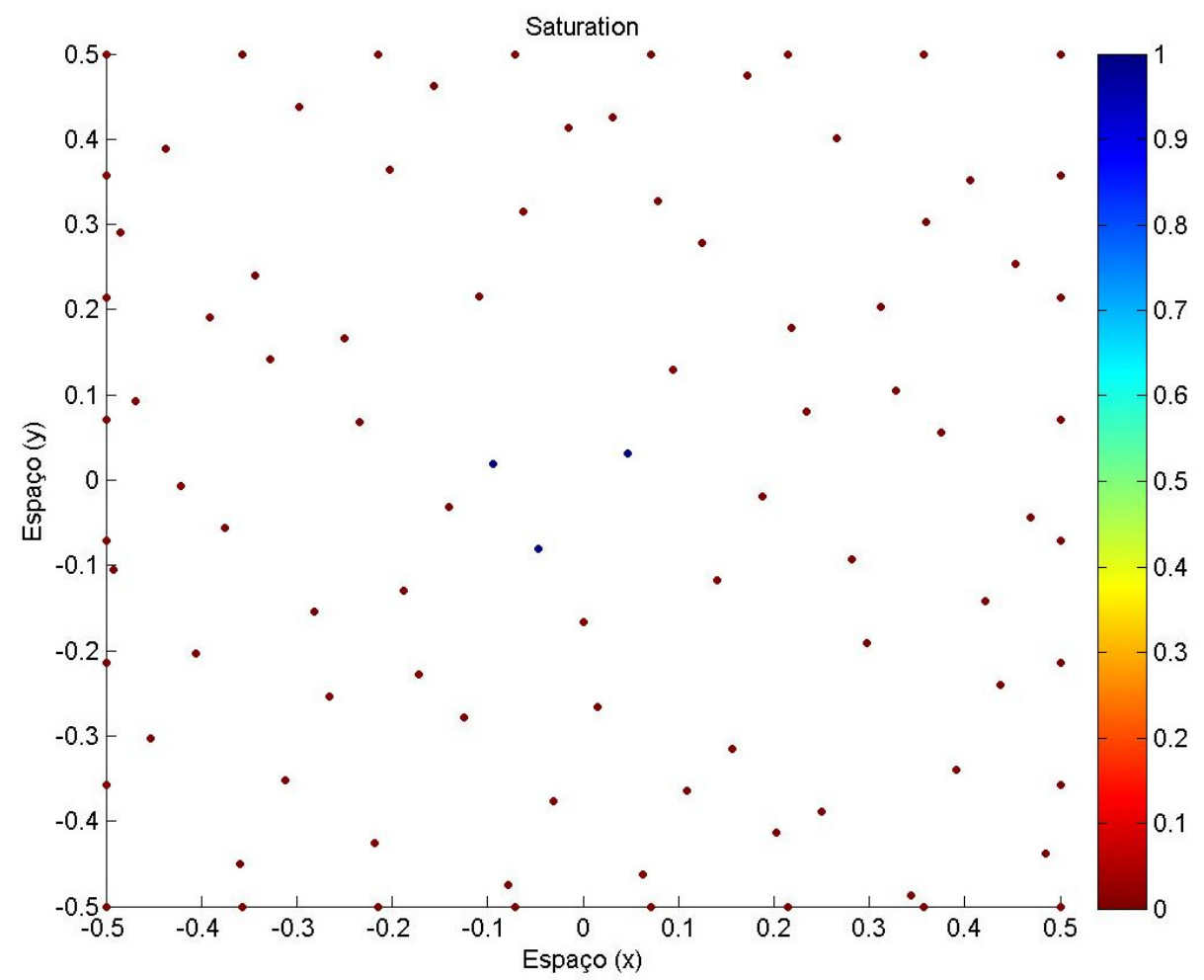

Figura 48: Saturação do modelo adaptativo do problema five spot, utilizando pontos Voronoi $t=1$

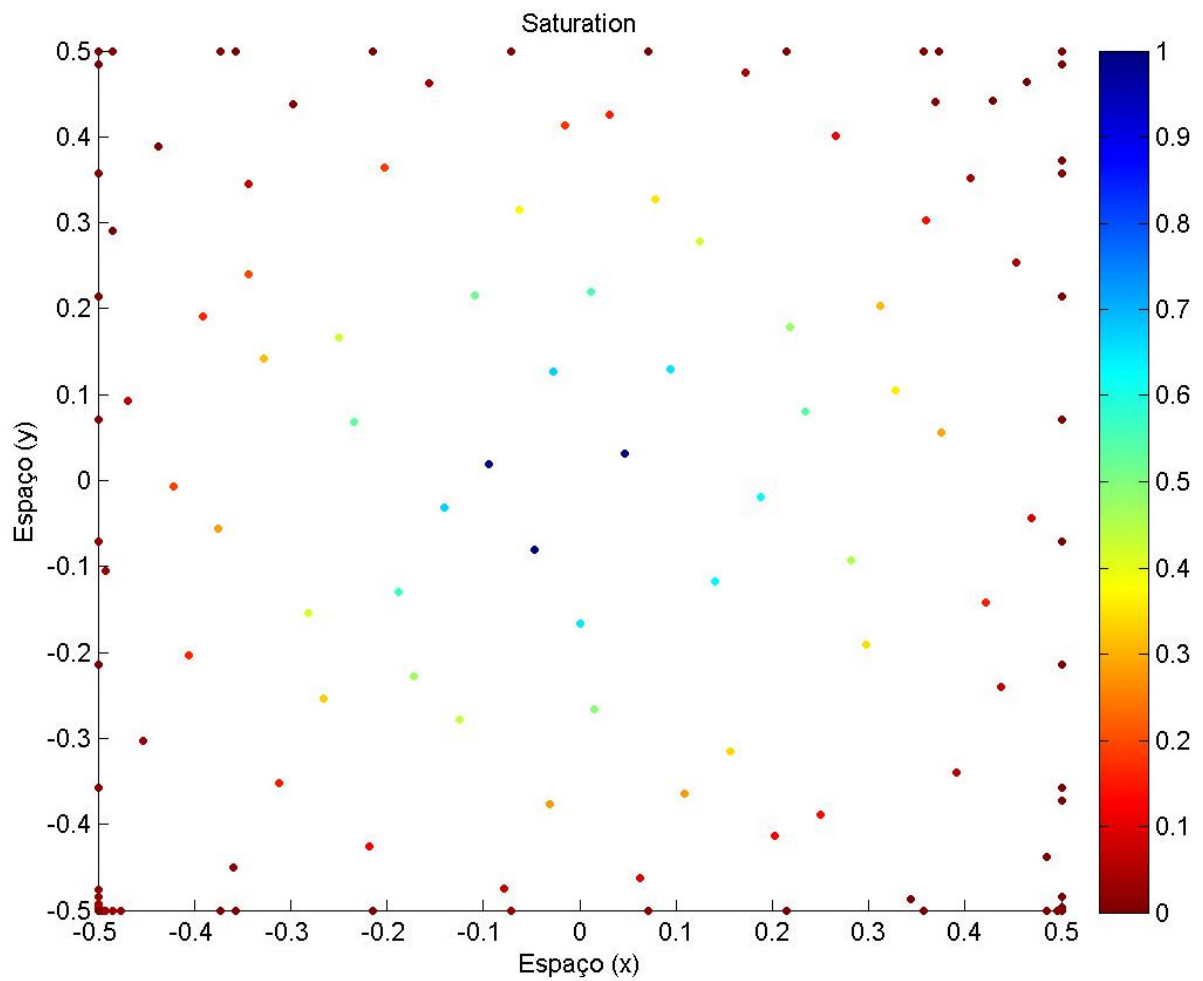

Figura 49: Saturação do modelo adaptativo do problema five spot, utilizando pontos Voronoi $t=8$ 


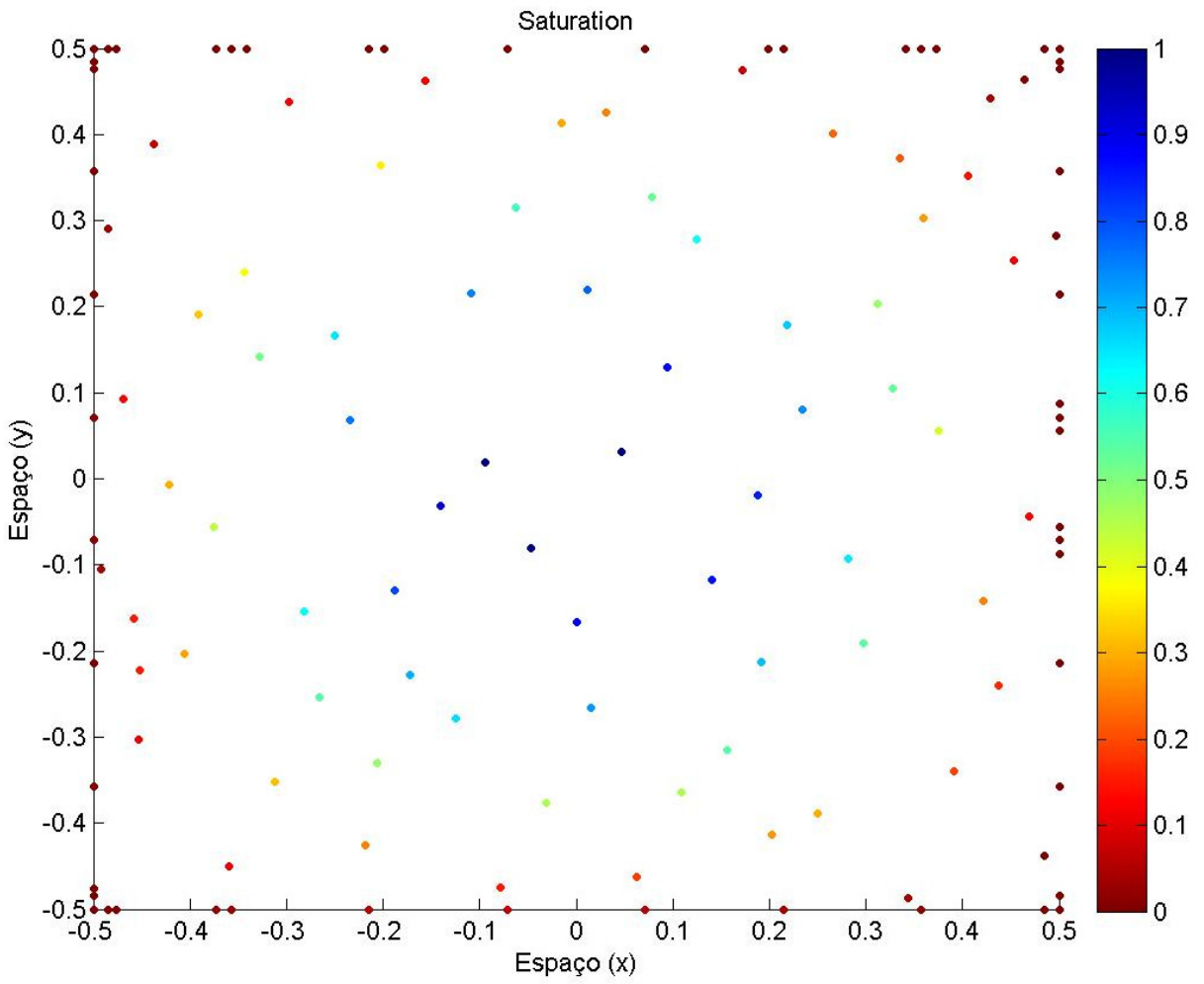

Figura 50: Saturação do modelo adaptativo do problema five spot, utilizando pontos Voronoi $t=75$

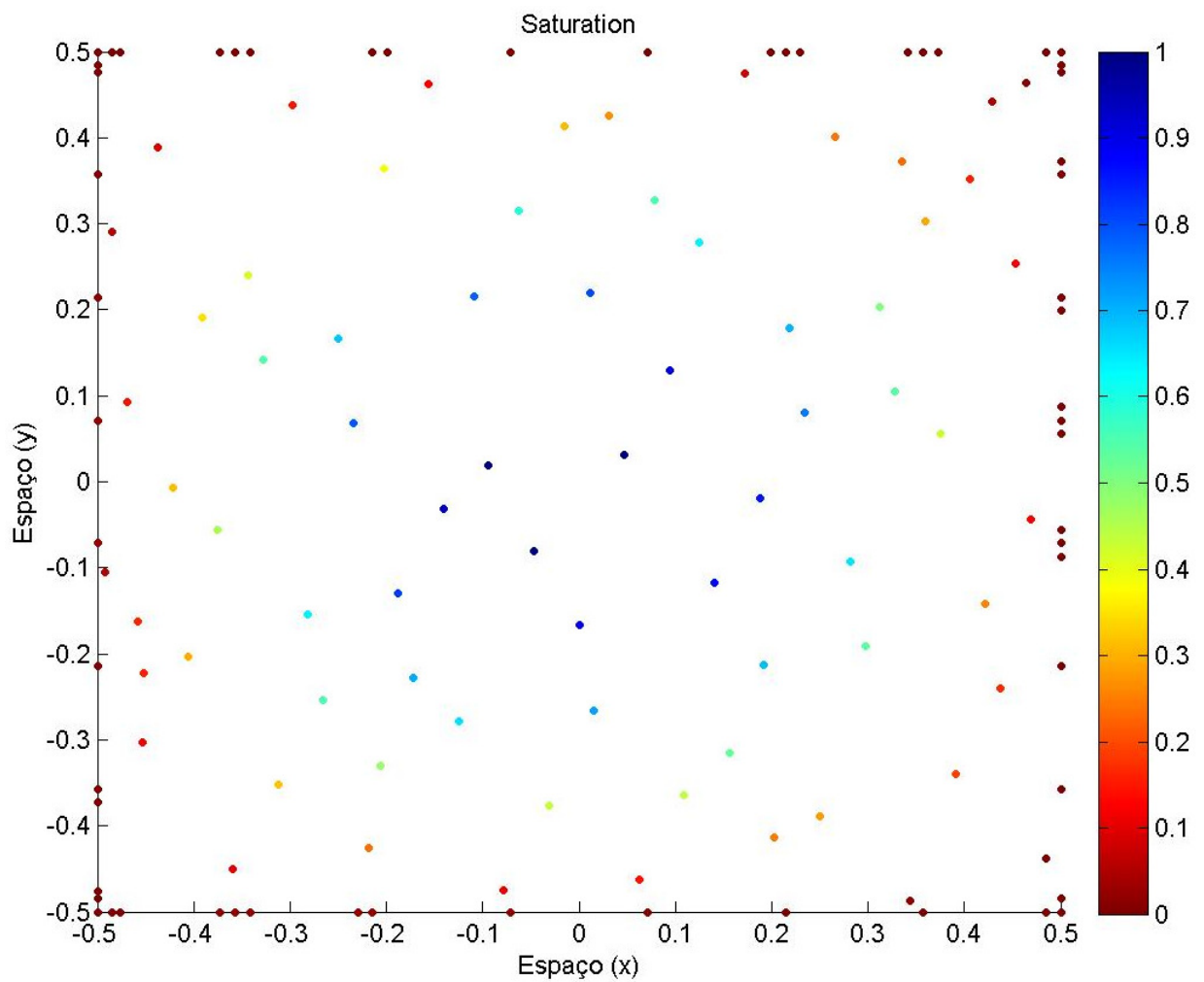

Figura 51: Saturação do modelo adaptativo do problema five spot, utilizando pontos Voronoi

$$
t=179
$$


A seguir serão apresentados os resultados do novo modelo adaptativo de advecção para problemas de fluxo bifásico de fluidos incompressíveis e imiscíveis em meios porosos heterogêneos tridimensionais.

\section{3. \\ Resultados do novo modelo bifásico imiscível e incompressível num meio poroso heterogêneo em domínio tridimensional.}

Nesta seção serão apresentadas as simulações numéricas obtidas com o novo modelo de advecção bifásico imiscível e incompressível utilizando tanto um meio poroso homogêneo como heterogêneo em um domínio computacional tridimensional, conforme proposta apresentada no capitulo 5, do problema clássico de reservatório de petróleo five spot. Esta pesquisa apresentará os resultados desprezando todos os efeitos gravitacionais. Portanto o objetivo dessa seção é analisar, validar e comparar os modelos feitos em uma geometria regular e uma geometria meshfree resultando assim em uma análise das principais vantagens e desvantagem que cada uma apresenta.

O modelo exemplo utilizado neste trabalho foi o modelo SPE 10 Model 2 (Christie e Blunt, 2001), para simulação de reservatório heterogêneo, onde as permeabilidades variam de forma análoga nas três direções isto é, são isotrópicas e a porosidade varia apenas espacialmente, conforme mencionado no capítulo 2 desta tese. É importante salientar que, neste caso, os dados de porosidade e de permeabilidade absoluta do modelo SPE 10 Model 2 foram extraídos com a base em amostras reais de um campo de um reservatório existente. Essas amostras são levadas a um laboratório para que as propriedades supracitadas sejam aferidas fazendo uma relação entre a escala do campo real e a escala de laboratório. Para garantir a representatividade, é necessário distribuir as propriedades obtidas, a partir da escala de laboratório, para a escala de campo, fazendo uso de técnicas de geoestatística. Assim, através de um histograma, é possível estabelecer uma distribuição que representa o reservatório. Posteriormente, o modelo numérico é comparado com a curva de produção real do campo. De maneira geral é necessário ajustar alguns parâmetros de forma que o modelo numérico possa representar fielmente os dados apresentados pelo campo; este processo é 
conhecido como ajuste de histórico. Portanto, uma comparação entre o resultado obtido com o modelo proposto e o Software IMEX, Apêndice A, não é pertinente, para o trabalho aqui desenvolvido. Cabe ressaltar que o processo descrito anteriormente faz uso de um grid de diferenças finitas regular e fixo.

A porosidade é uma função escalar que depende das coordenadas espaciais e esta função é de extrema importância para simulações de meios porosos heterogêneos ou em simulações que considerem os efeitos do acoplamento geomecânico (Murad e Loula, 1992; Peaceman, 1977; Hasle et al, 2007).

O modelo original SPE Model 2 (3D) do SPE66599 (Christie e Blunt, 2001) trata um problema tridimensional, fazendo uso de uma malha cartesiana de $60 \mathrm{x}$ 220 x 85 (1.122.000 células). O espaçamento em cada direção é dado por:

$$
\begin{aligned}
& \text { Deltax }=20 \mathrm{ft} \\
& \text { Deltay }=10 \mathrm{ft} \\
& \text { Deltaz }=2 \mathrm{ft}
\end{aligned}
$$

Então pode-se dizer que a dimensão física do reservatório, respectivamente é dada por:

$$
[6022085] \times[20102] \times(0.3048)=[365.76 ; 670.56 ; 51.82] \text { metros }
$$

Para determinar os valores de porosidade e de permeabilidade absoluta adaptada para uma geometria meshfree foi usado o dado original fornecido pelo SPE 10 Model 2, apresentado neste trabalho. As equações 6.21, 6.22, 6.23 e 6.24 determinam as novas posições para obter os valores de porosidade e de permeabilidade absoluta de cada ponto do modelo, isto é:

$$
\text { Posicao_ } i=\frac{\left(N_{x}-1\right)\left(x-\frac{\operatorname{Dim} X}{2}\right)+N_{x} \operatorname{Dim} X}{\operatorname{Dim} X}
$$




$$
\text { Posicao }_{-} j=\frac{\left(N_{y}-1\right)\left(y-\frac{\operatorname{Dim} Y}{2}\right)+N_{y} \operatorname{Dim} Y}{\operatorname{Dim} Y}
$$

$\mathrm{e}$

$$
\text { Posicao_k }_{-}=\frac{\left(1-N_{z}\right)(z-(-\operatorname{Dim} Z))+(-\operatorname{Dim} Z)}{(-\operatorname{Dim} Z)} \text {. }
$$

Então

$$
\begin{aligned}
& \text { SPELine }=\left(\text { Posicao }_{-} j-1\right) N_{x}+\text { Posicao_i }{ }^{\prime} . \\
& +(\text { Posicao_k }-1) N_{x} N_{y}
\end{aligned}
$$

Onde SPELine representa a nova posição dos valores do campo de porosidade e do campo de permeabilidade absoluta. As posições Posicao ${ }_{i} i$, Posicao $_{-} j \mathrm{e}$ Posicao _ $k$ são as posições nas direções $x y$ e $z$ enquanto que $N_{x}, N_{y}$ e $N_{z}$ são os tamanhos máximos nas direções $x, y$ e $z$. Além disso, $\operatorname{Dim} X, \operatorname{Dim} Y$ e $\operatorname{Dim} Z$ são as dimensões físicas do reservatório nas três direções.

Em relação às 85 camadas deste modelo, as 35 camadas superiores são do tipo "formação Tabert", que pode ser entendido como um terreno arenoso composto basicamente por arenito de baixa compactação e as 50 últimas camadas são do tipo "Upper Ness" compostas de um material de alta compactação. A formação "Upper Ness" é uma formação fluvial, que é encontrada em geral em terrenos abaixo de um rio e composto por uma sequência de arenitos, siltitos e folheios (Deegan e Scull, 1977). Na camada de "Upper Ness" há caminhos permeáveis que são os arenitos e impermeáveis que são as rochas. Da Figura 52 à Figura 55, mostram a porosidade e a permeabilidade absoluta do meio heterogêneo, utilizando o modelo SPE 10 Model 2.

Inicialmente na seção 6.3.1 são apresentados os resultados do modelo proposto utilizando uma malha cartesiana regular em um meio poroso homogêneo e também em um meio poroso heterogêneo e na seção 6.3.2 são apresentados os resultados do modelo proposto usando um meio poroso heterogêneo com base nos métodos meshfree, conforme proposta apresentada. 


\section{Outros dados de entrada do modelo SPE 10, modelo 2 do SPE66599.}

- Viscosidades da água $=3 \times 10^{-4}$ e do óleo $=3 \times 10^{-3}$

- Densidade da água $=64 \mathrm{lb} / \mathrm{ft}^{3}$ e densidade do óleo $=53 \mathrm{lb} / \mathrm{ft}^{3}$

- Pressão inicial do reservatório $=6000$ psi

- Todos os poços são verticais sendo que: $\mathrm{I} 1=[600,1100] ; \mathrm{P} 1=[0,0]$;, $\mathrm{P} 2=[1200,0] ; \mathrm{P} 3=[1200,2200] ; \mathrm{P} 4=[0,2200]$.

- Razão de injeção = $5000 \mathrm{bbl} / \mathrm{dia}$

- Pressão máxima de injeção $=10000$ psi

Onde $I 1$ denota o poço de injeção e $P 1, P 2, P 3$ e $P 4$ denotem os poços de produção.

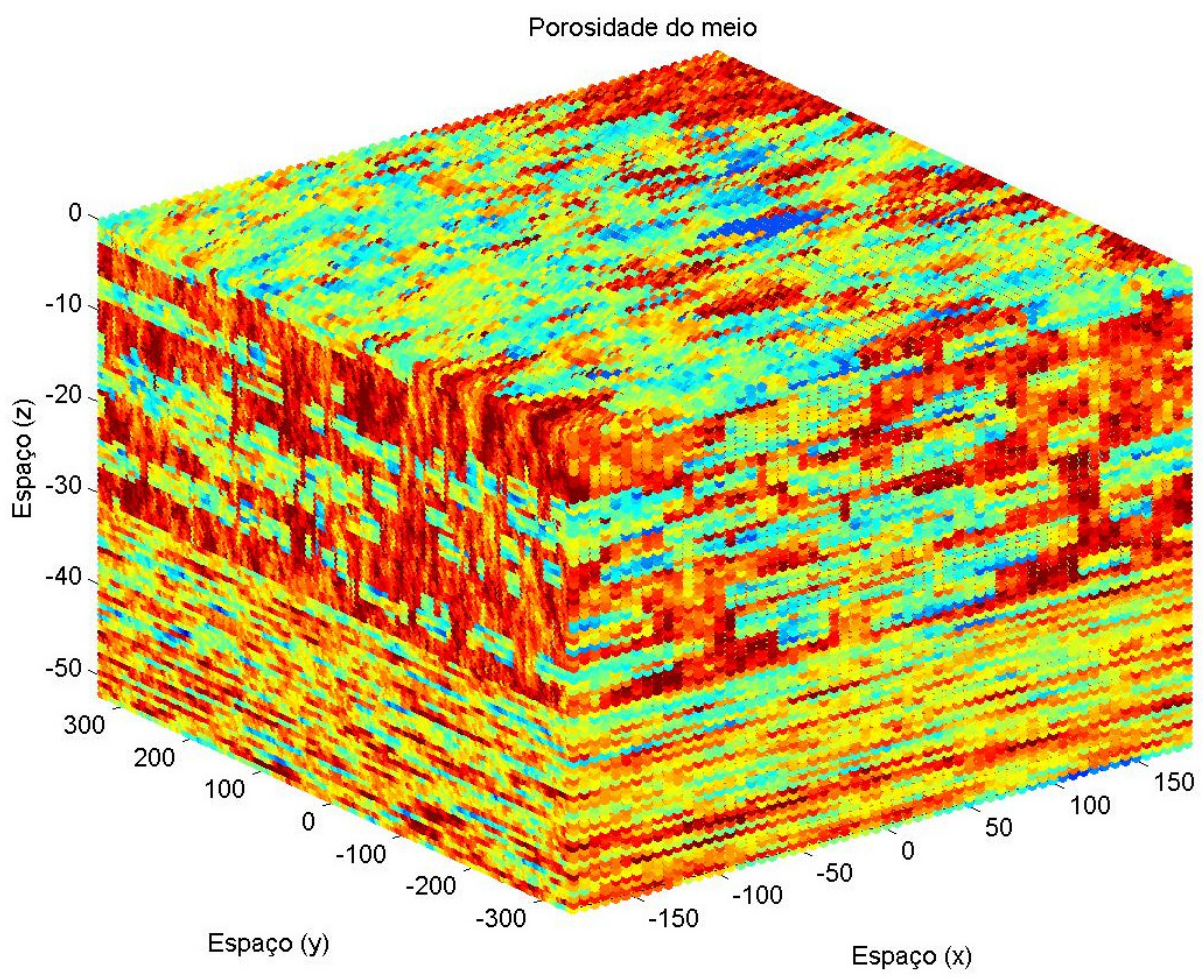

Figura 52: Porosidade do meio heterogêneo SPE 10, modelo 2. 


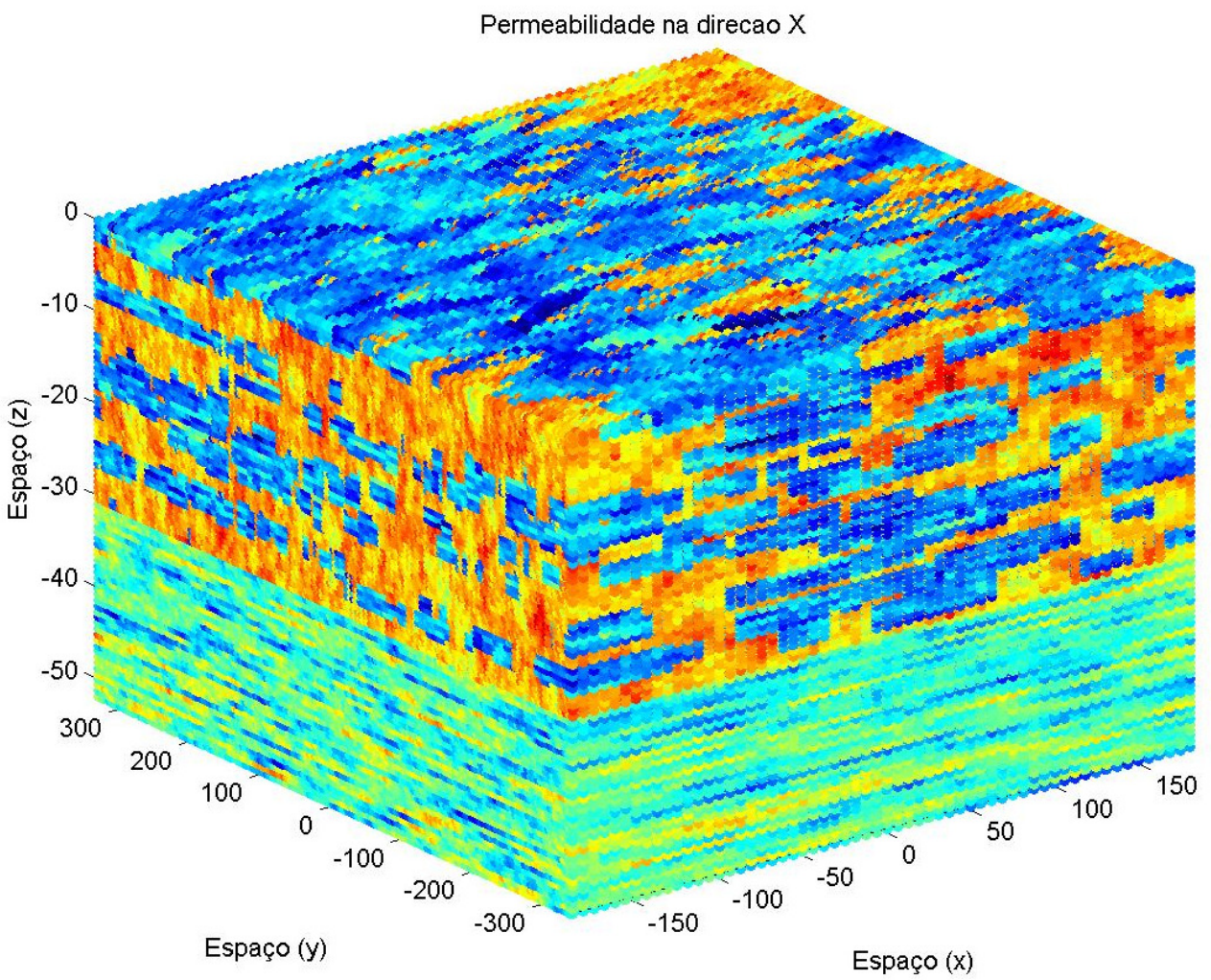

Figura 53: Visão do logaritmo da permeabilidade absoluta do meio poroso heterogêneo

SPE 10, modelo 2 do SPE66599 na direção X.

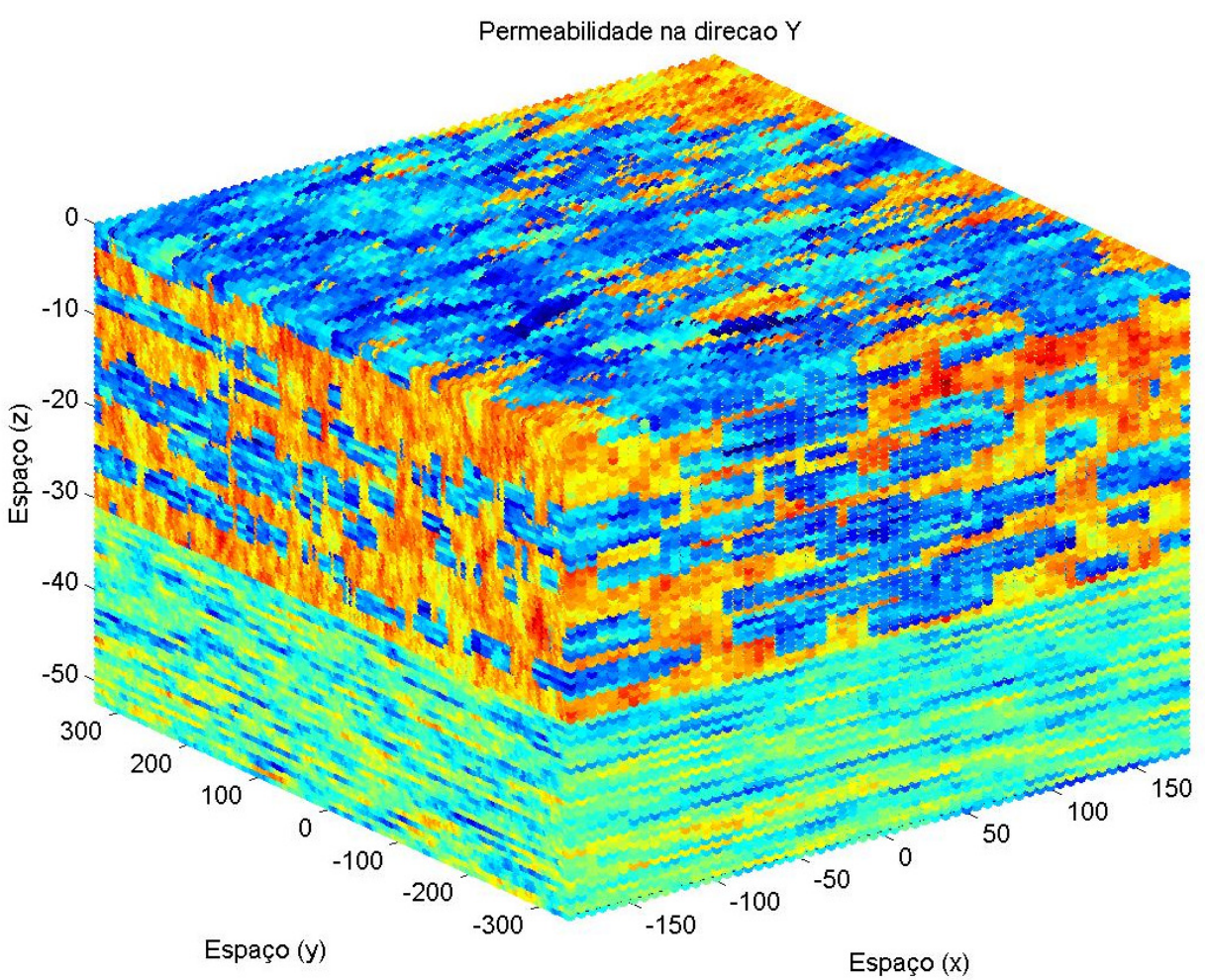

Figura 54: Visão do logaritmo da permeabilidade absoluta do meio poroso heterogêneo SPE 10, modelo 2 do SPE66599 na direção Y. 


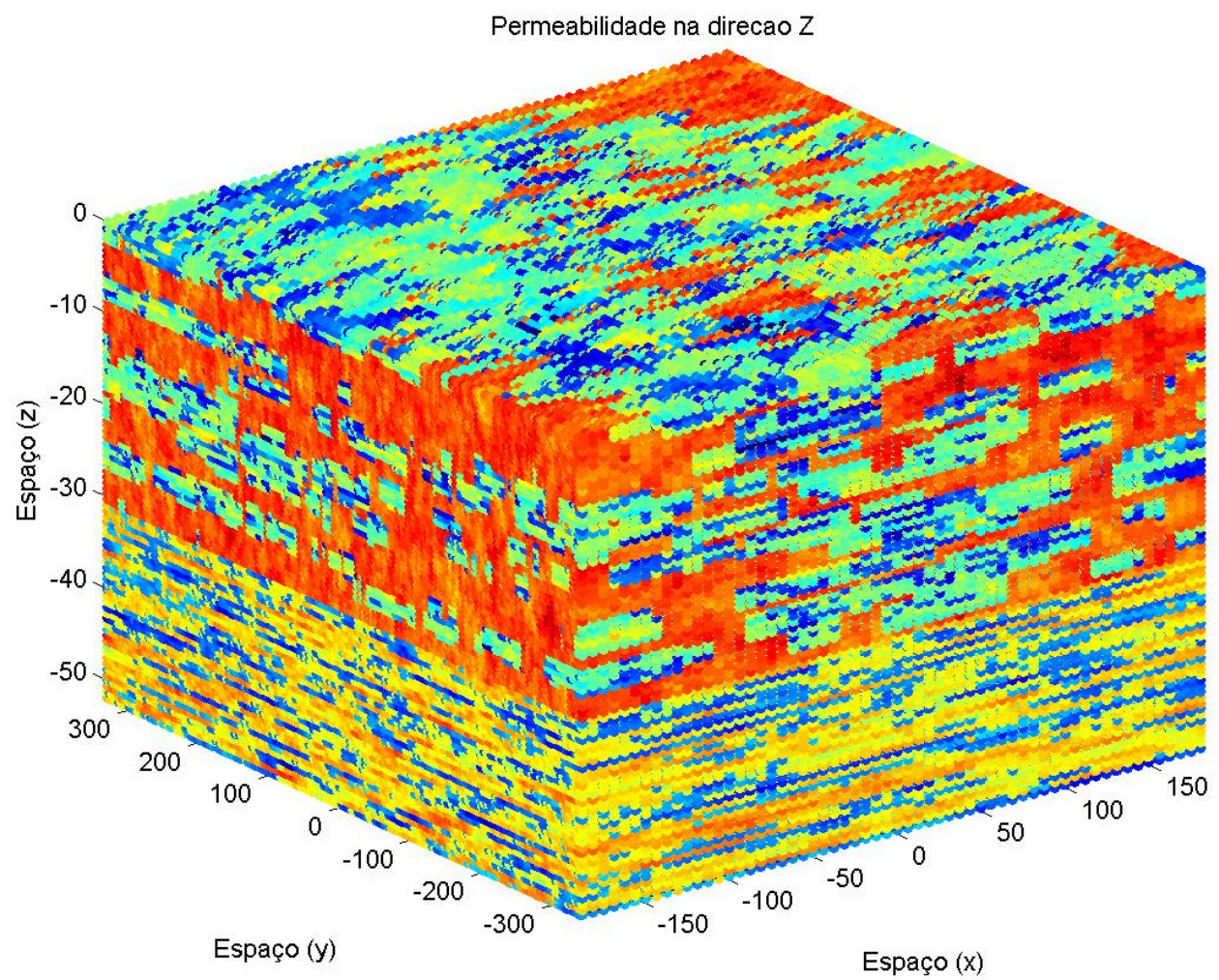

Figura 55: Visão do logaritmo da permeabilidade absoluta do meio poroso heterogêneo SPE 10, modelo 2 do SPE66599 na direção Z.

A seguir, serão apresentados os resultados do modelo de advecção conforme proposto no capítulo 5 .

\subsection{1. \\ Resultados do Novo Modelo Proposto Utilizando uma Geometria Regular}

Nesta seção serão apresentados alguns resultados do modelo de advecção bifásico imiscível e incompressível utilizando um meio poroso homogêneo e heterogêneo usando uma geometria regular. Inicialmente, nesta seção serão apresentadas as simulações de saturação em um reservatório utilizando um meio poroso homogêneo e em seguida a saturação usando um meio poroso heterogêneo, nos dois casos, fazendo uso de uma geometria regular.

Os resultados da Figura 56 à Figura 59 foram simulados utilizando a simplificação dos dados heterogêneos para dados homogêneos, ou seja, fazendo com que a permeabilidade absoluta e a porosidade fossem constantes para todo o 
reservatório. As simulações foram feitas nos instantes de tempo $T=1, T=200$, $T=300$ e $T=400$, utilizando uma malha regular composta por [60, 220, 17], isto é composta por 224400 células. Ambas as figuras representam a variação da saturação das fases agua e óleo em meio poroso homogêneo, sendo que a cor vermelha representa o fluido puramente água, ou seja, $U=0.2$ e a cor azul representa o fluido puramente óleo, isto é, $U=0.8$. A combinação das cores entre vermelha e azul escuro mostra a variação da saturação $U$, ao longo do tempo.

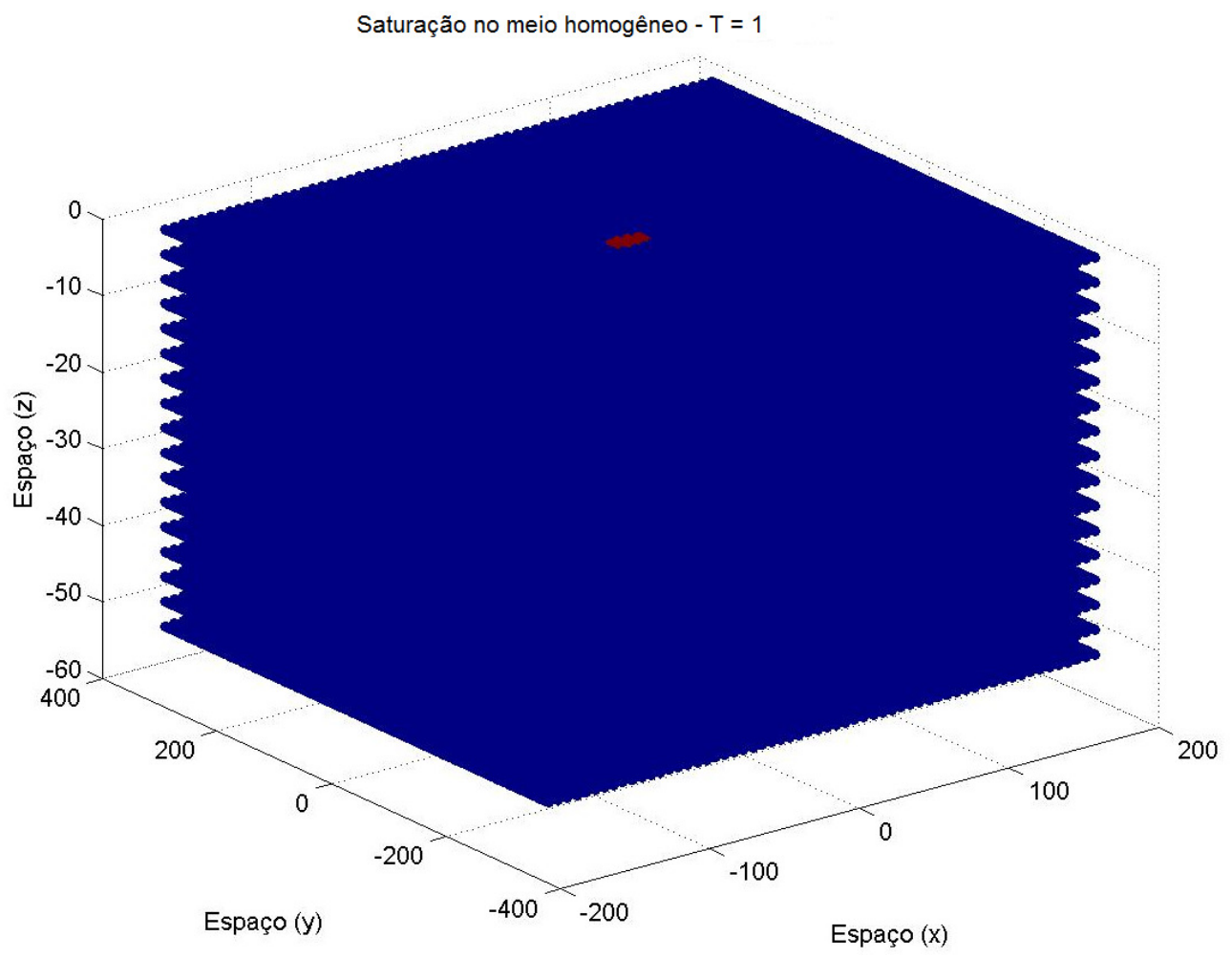

Figura 56: Saturação no meio poroso homogêneo em $T=1$. 


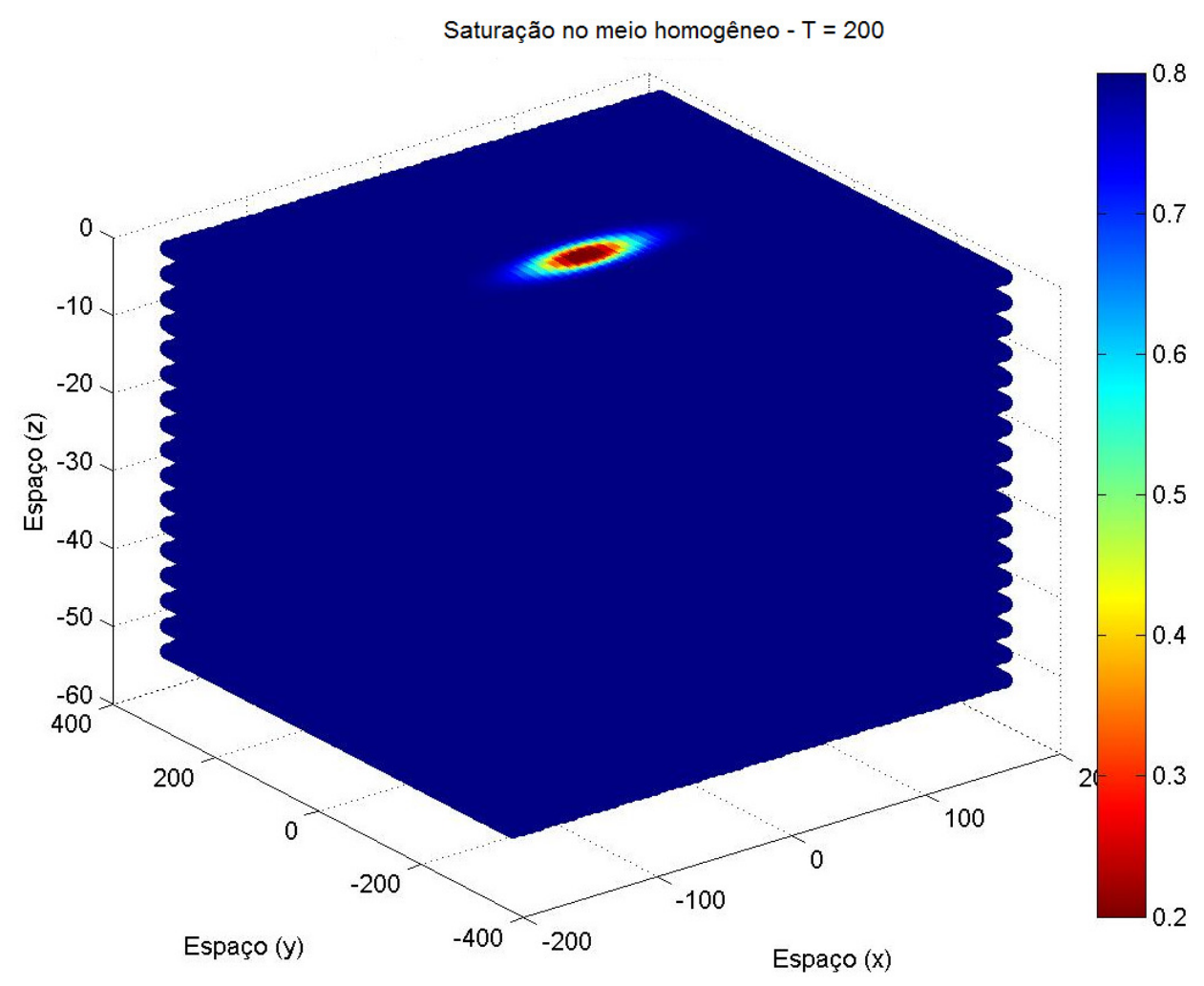

Figura 57: Saturação no meio poroso homogêneo em $T=200$. 


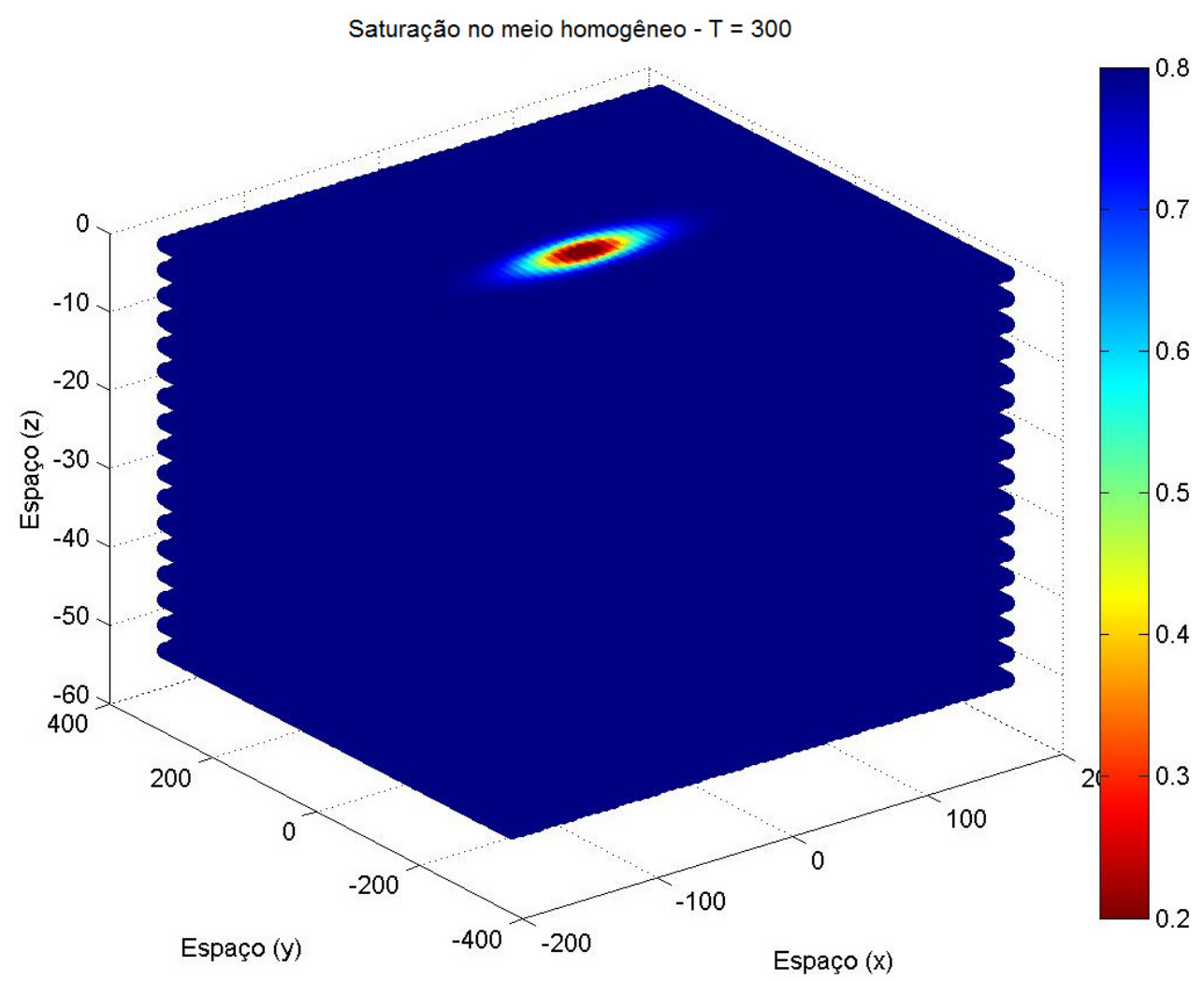

Figura 58: Saturação no meio poroso homogêneo em $T=300$. 


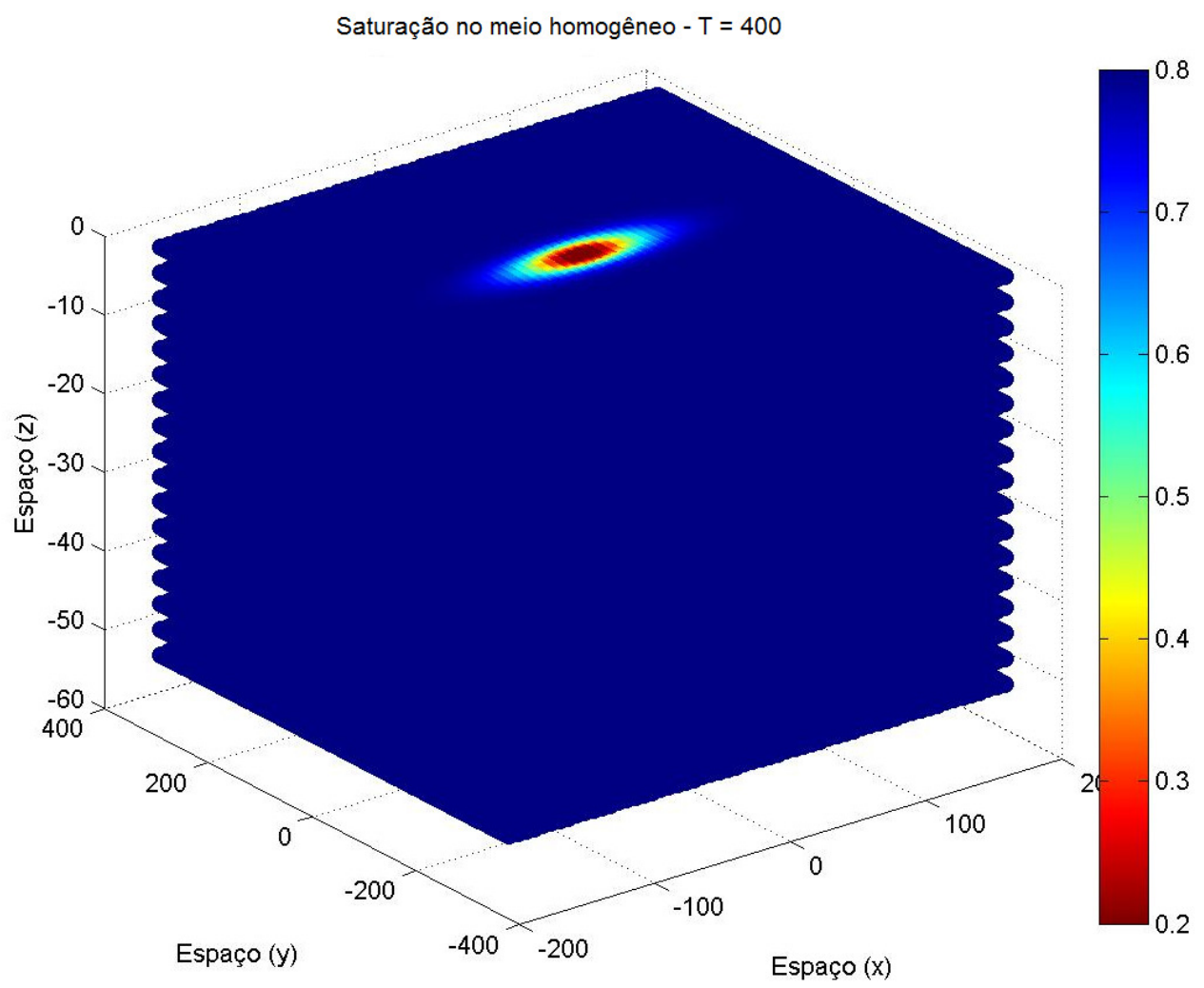

Figura 59: Saturação no meio poroso homogêneo em $T=400$.

A interface água / óleo está se deslocando do centro para os quatro poços das extremidades, o mesmo efeito visto no modelo bidimensional.

A seguir, serão apresentados os resultados em um meio poroso heterogêneo usando também uma geometria regular. Portanto, da Figura 60 à Figura 64 exibem os principais resultados obtidos da saturação, tendo como base um meio poroso heterogêneo. Foram realizadas simulações para os instantes de tempo $T=1, T=200, T=300, T=400$ e $T=2000$, utilizando uma malha composta por $[60,220,1]$, ou seja, cartesianamente composta por 13200 células. 


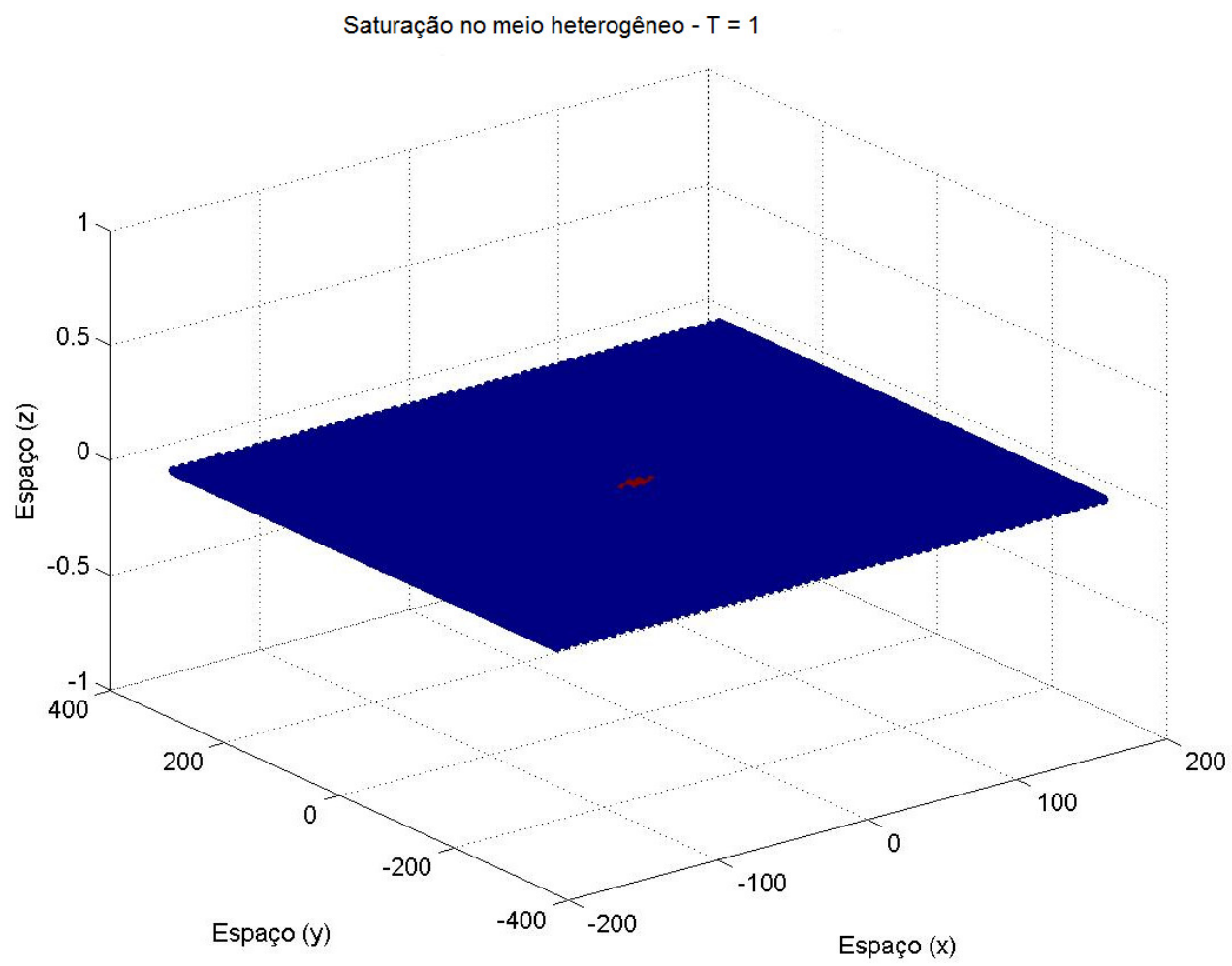

Figura 60: Saturação no meio poroso heterogêneo em $T=1$.

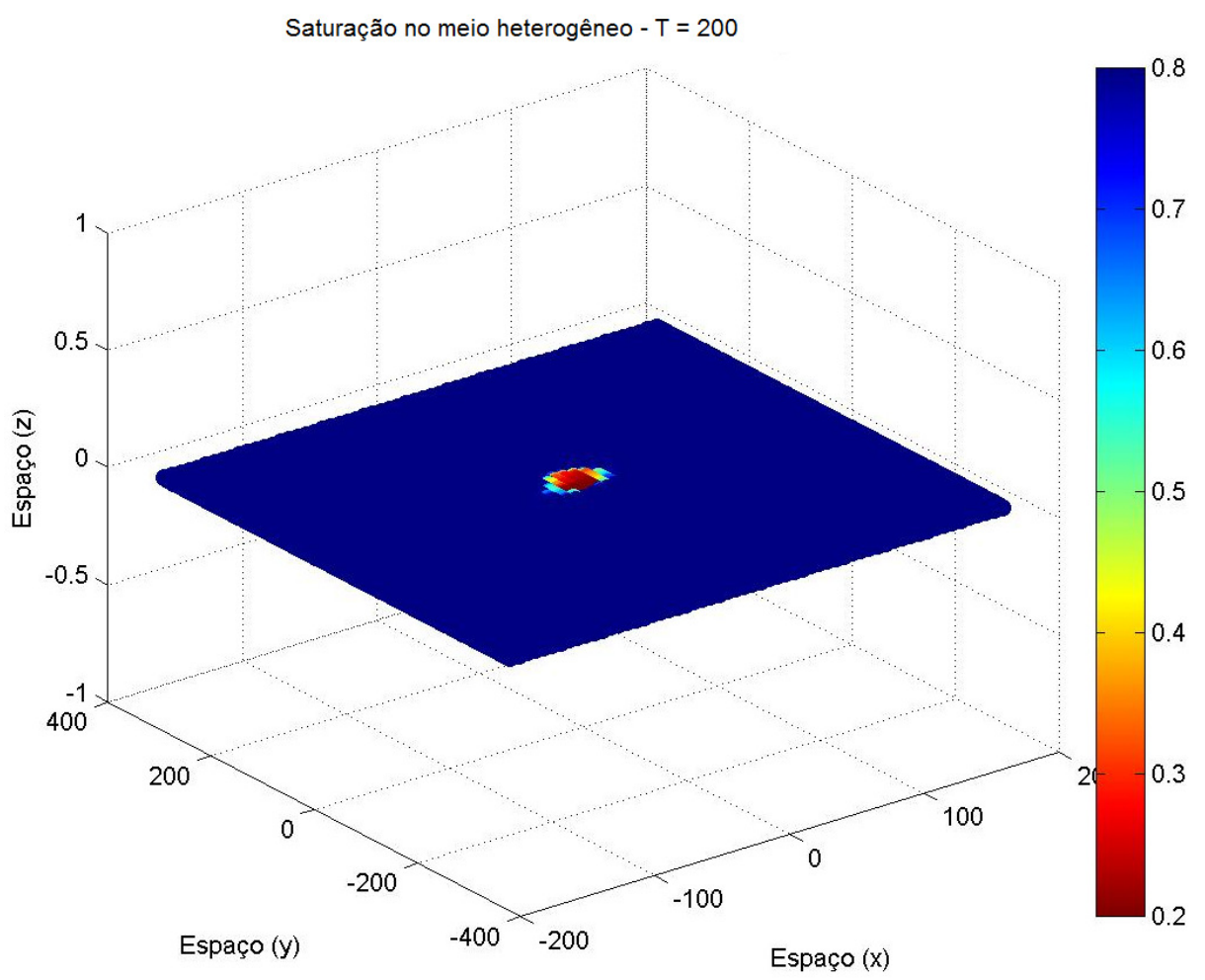

Figura 61: Saturação no meio poroso heterogêneo em T $=200$. 


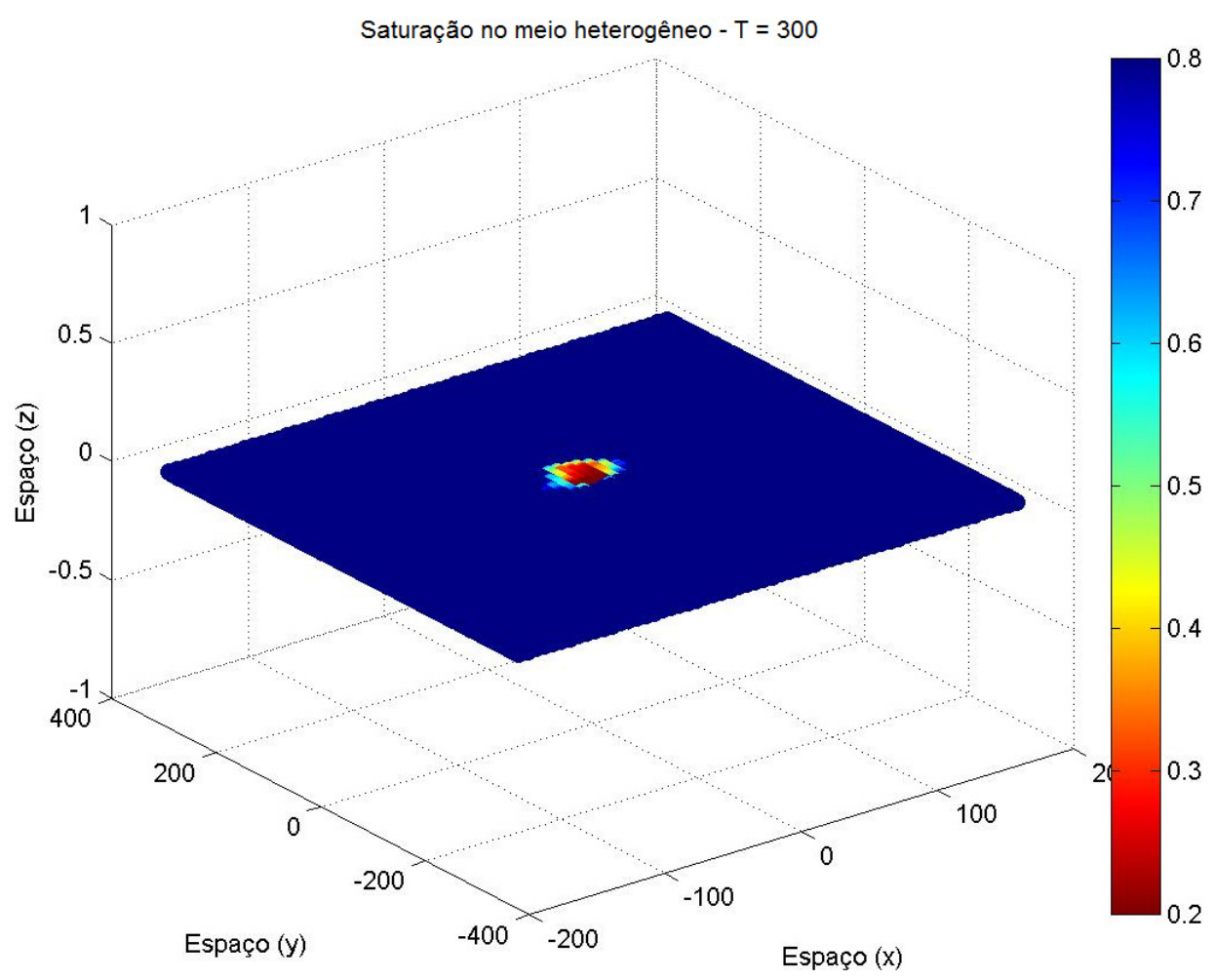

Figura 62: Saturação no meio poroso heterogêneo em $T=300$.

Apesar de usar uma malha pouco refinada é possível observar um deslocamento da propagação da frente de onda entre a fase água e a fase óleo do poço de injeção para os quatro poços de produção. Note que da Figura 60 à Figura 64 há uma variação significativa no deslocamento do fluído água em relação a fluído óleo que representa fisicamente o comportamento do problema five spot em um reservatório de petróleo em um meio poroso heterogêneo. Neste exemplo que trata de um meio poroso heterogêneo percebe-se também que há uma variação das propriedades da rocha, segundo o comportamento apresentado por ambos.

Uma grande diferença no comportamento da Figura 56 à Figura 59 em relação às da Figura 60 à Figura 64. Essa diferença é devida principalmente à variação da porosidade e da permeabilidade absoluta do meio heterogêneo. 


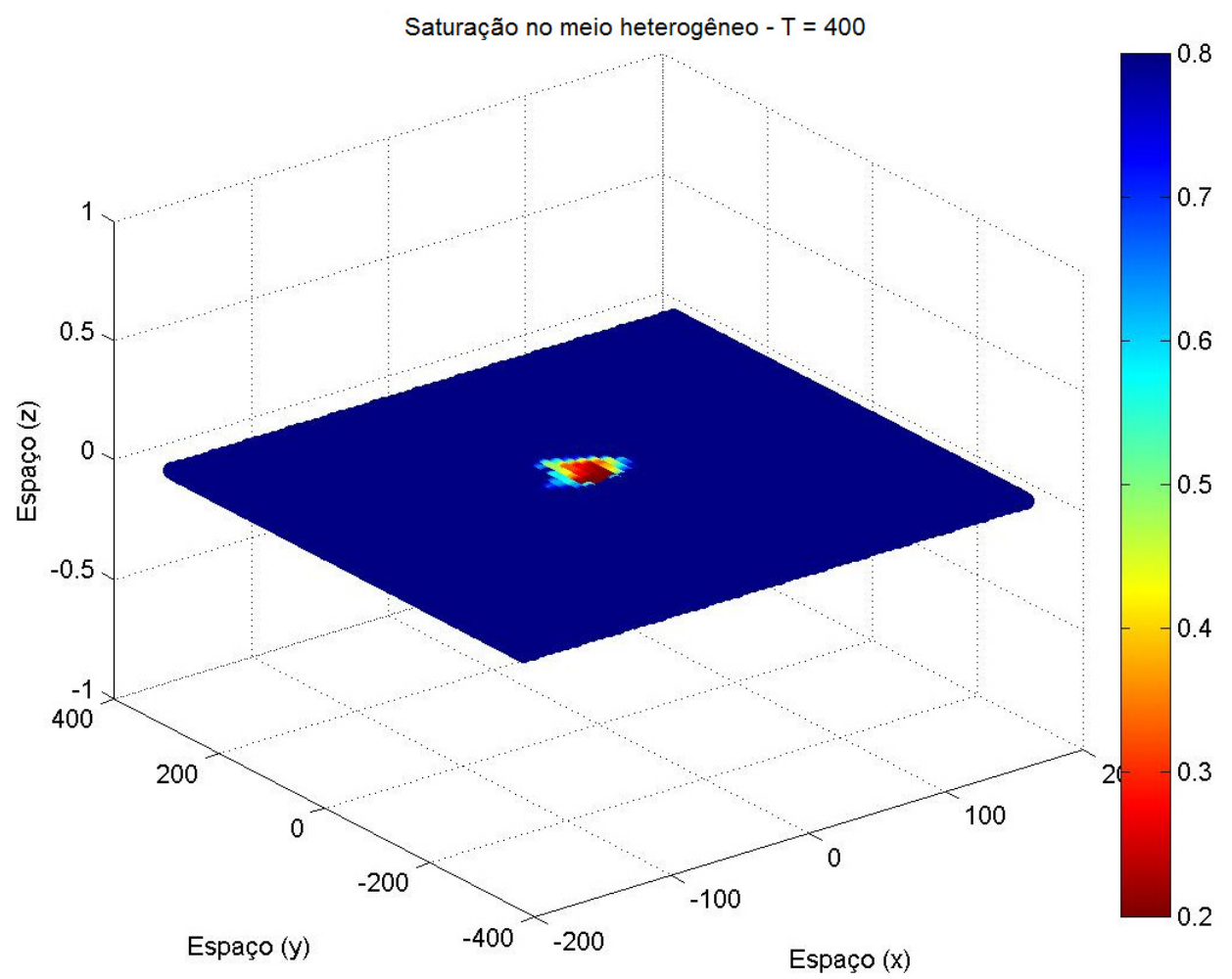

Figura 63: Saturação no meio poroso heterogêneo em $T=400$.

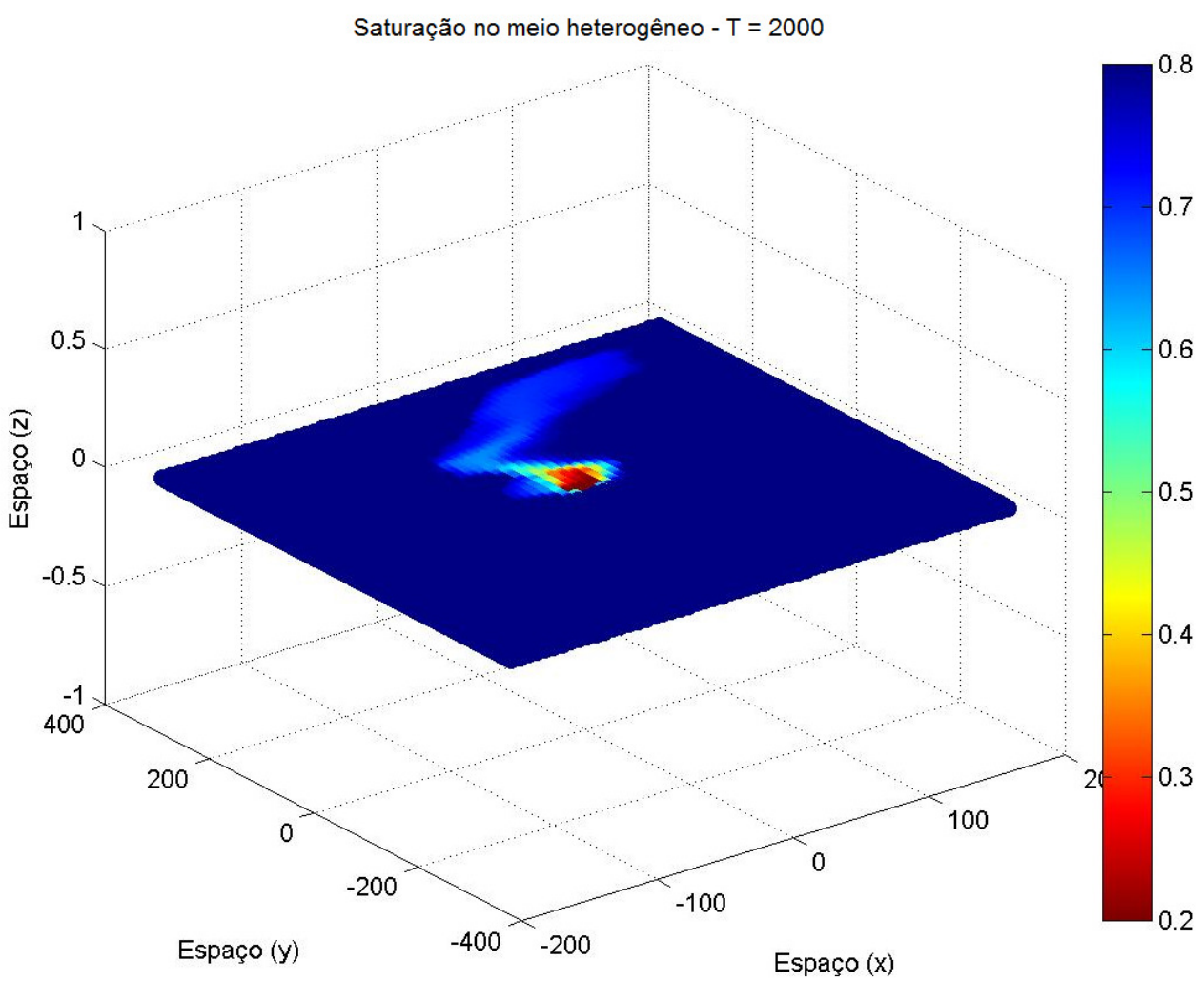

Figura 64: Saturação no meio poroso heterogêneo em $T=2000$. 
A seguir, serão apresentados os resultados do modelo proposto utilizando uma geometria meshfree.

\subsection{2. Resultados do novo modelo proposto utilizando uma geometria meshfree}

Nesta seção serão exibidos alguns resultados do modelo proposto de advecção bifásico imiscível e incompressível utilizando um meio poroso heterogêneo, sendo que agora utilizando uma geometria meshfree. É importante salientar, novamente, que a porosidade e a permeabilidade absoluta do meio poroso heterogêneo do modelo SPE 10 Model 2 foram adaptadas para uma geometria generalizada de acordo com as coordenadas espaciais e as dimensões físicas do modelo, já mencionado anteriormente, como demostrado nas equações $6.21,6.22,6.23$ e 6.24 .

A seguir, serão apresentados os resultados do modelo proposto utilizando uma geometria meshfree. As figuras nesta seção foram geradas utilizando 224400 nós randômicos, o mesmo número nós da grade regular. 


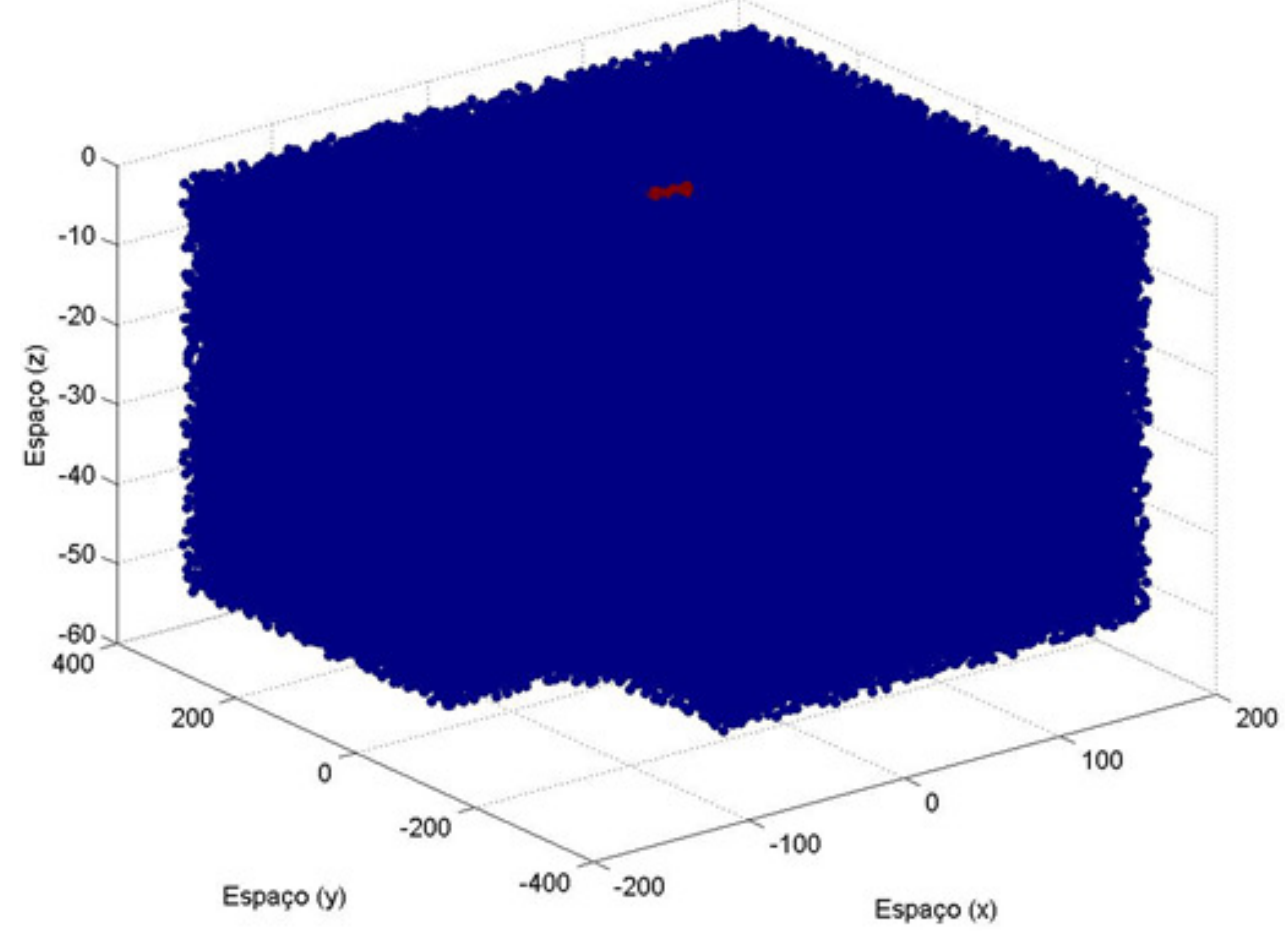

Figura 65: Saturação no meio poroso heterogêneo em $T=1$. 


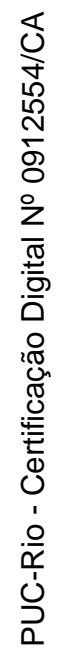

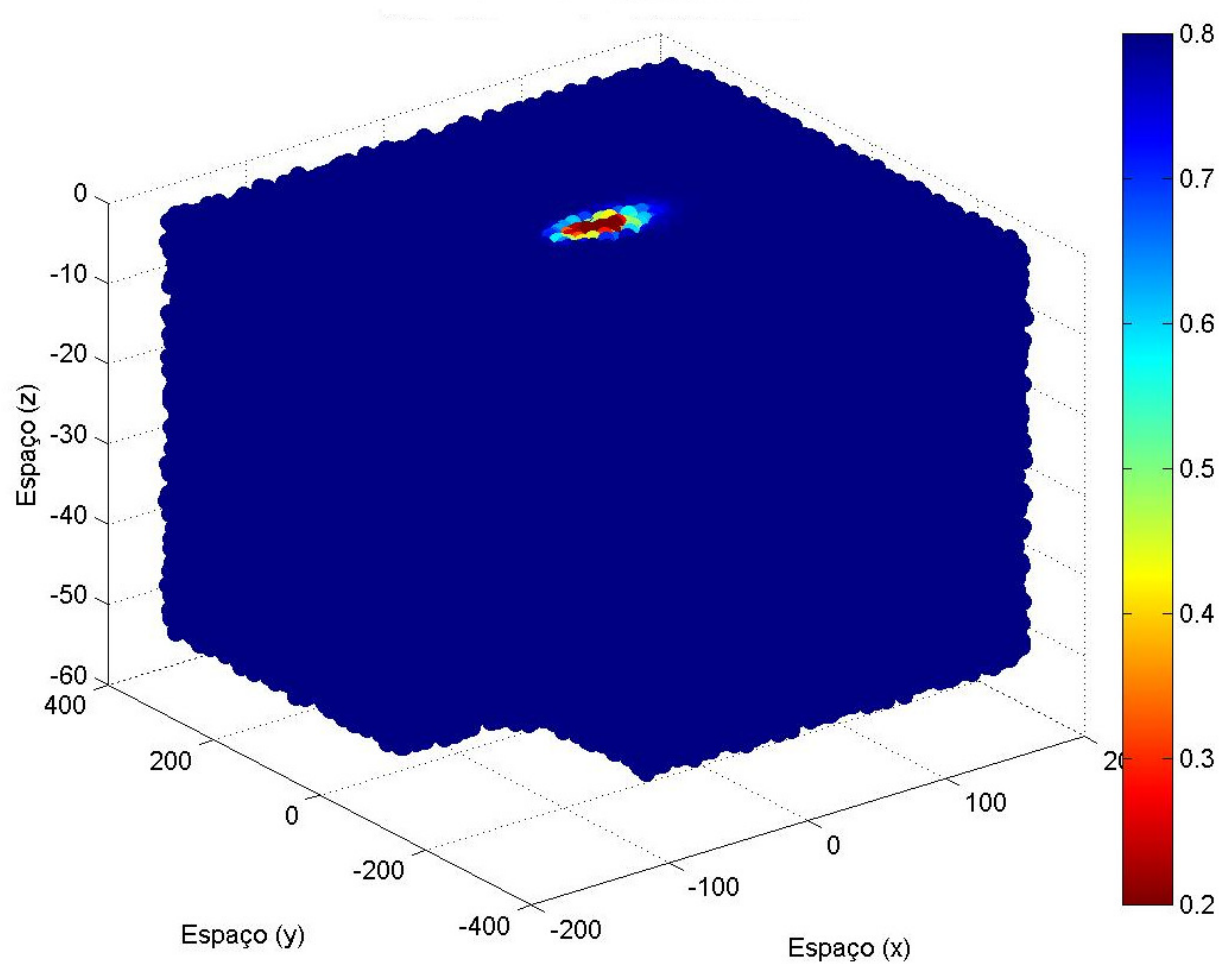

Figura 66: Saturação no meio poroso heterogêneo em $T=100$.

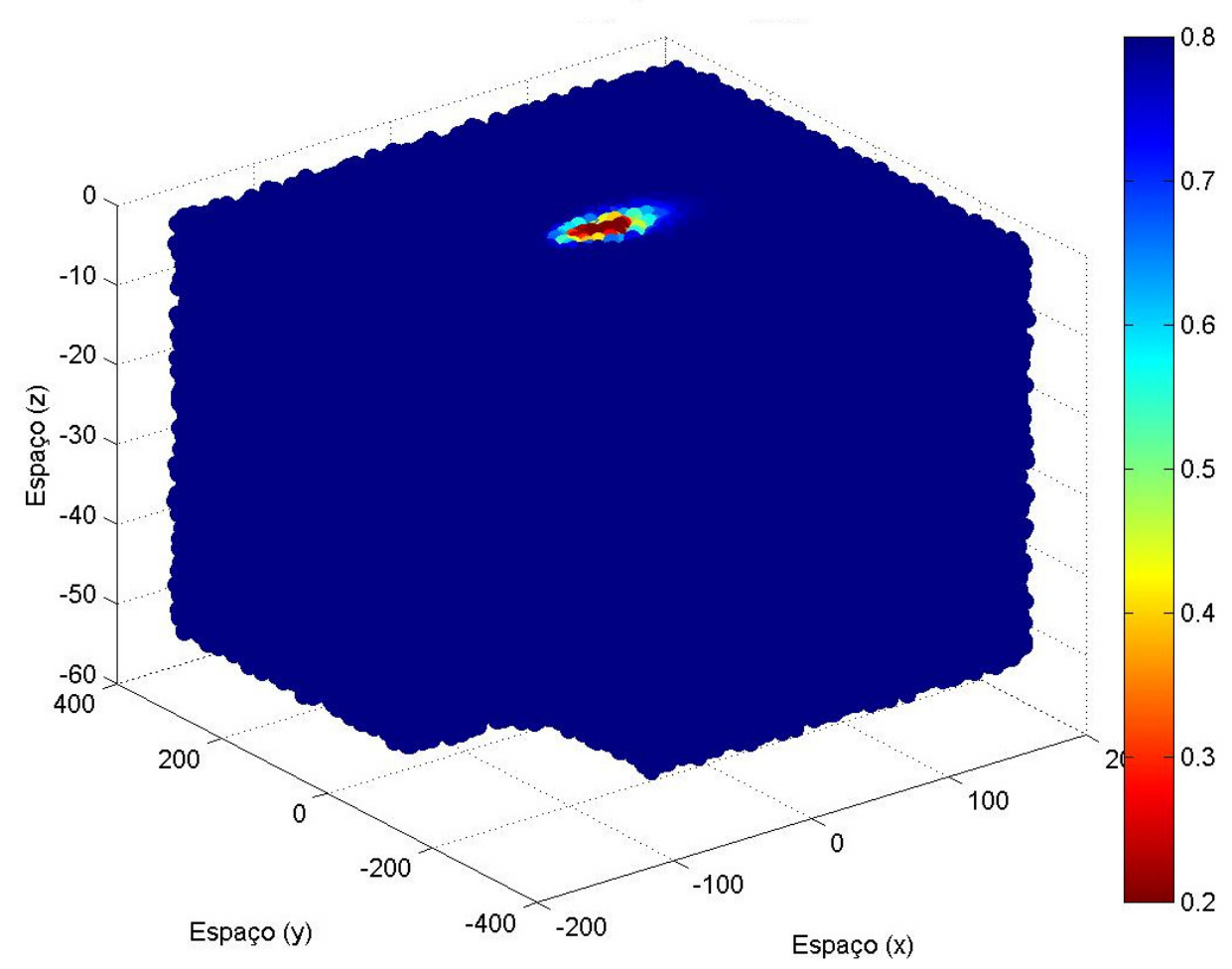

Figura 67: Saturação no meio poroso heterogêneo em $T=150$. 


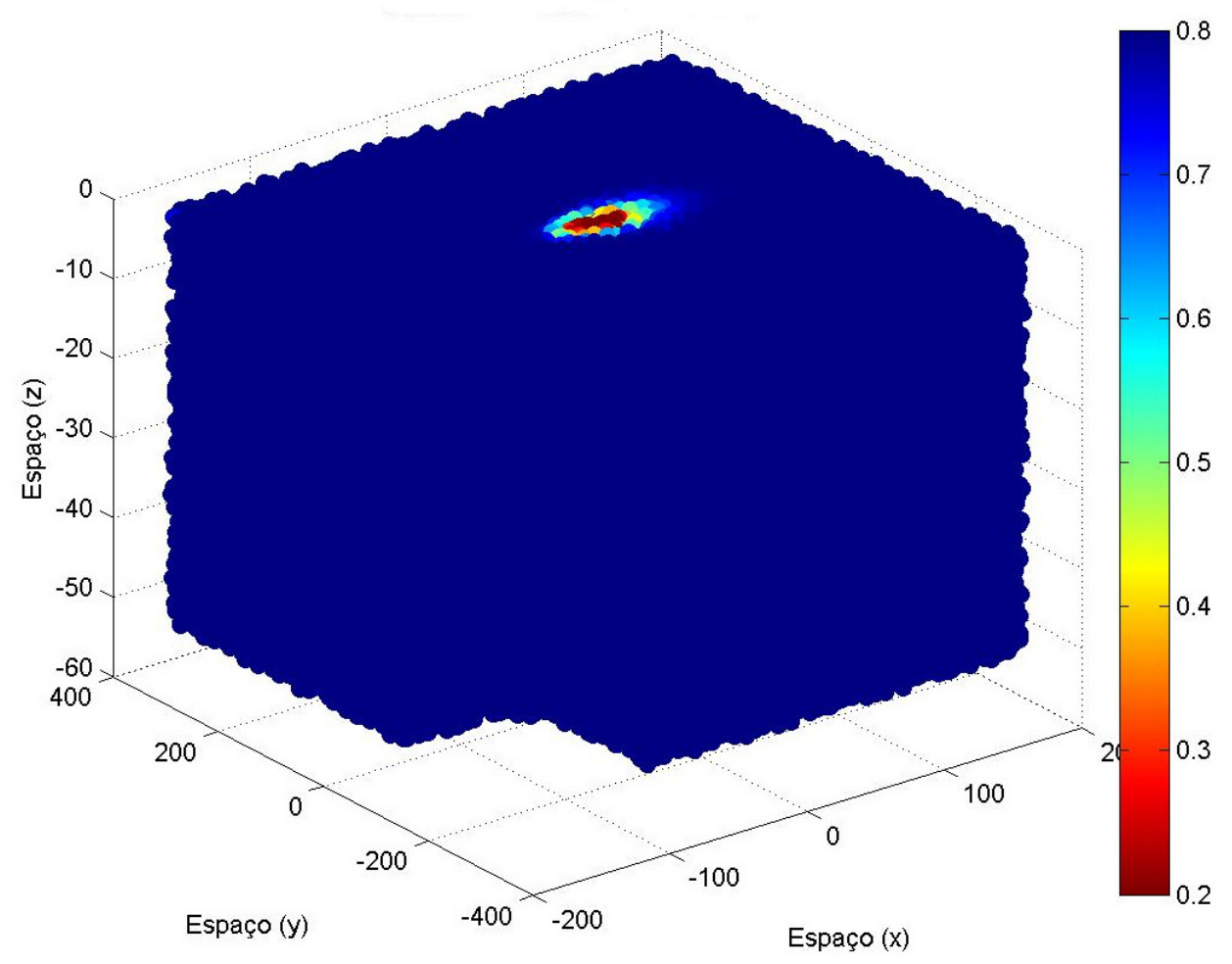

Figura 68: Saturação no meio poroso heterogêneo em $T=200$. 


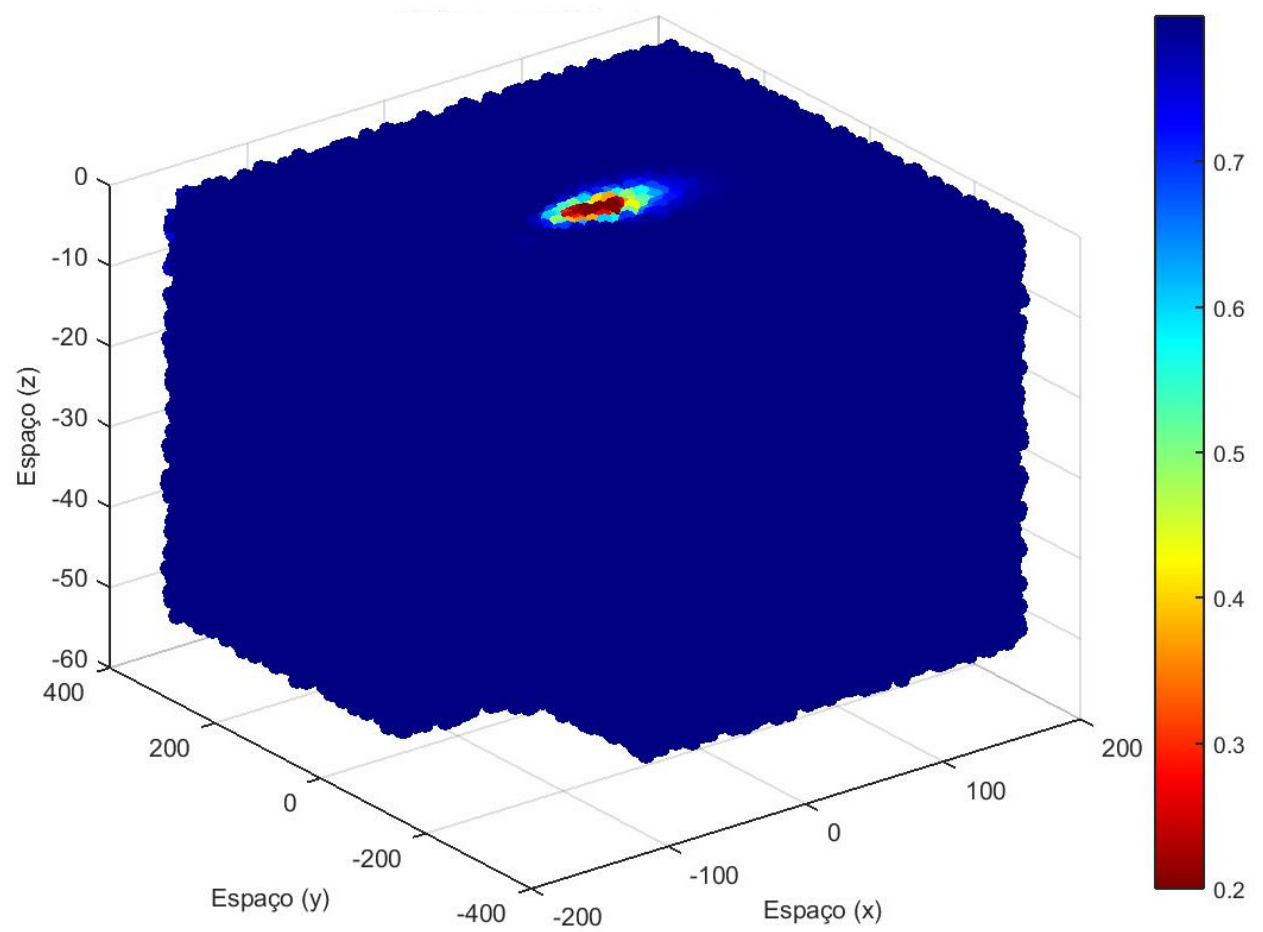

Figura 69: Saturação no meio poroso heterogêneo em $T=250$.

Da Figura 65 à Figura 69 são exibidas a saturação do meio poroso heterogêneo nos instantes de tempo $T=1, T=100, T=150, T=200$ e $T=250$, utilizando uma geometria meshfree. Nestas figuras observa-se também uma variação considerável no deslocamento do fluído água em relação a fluído óleo que representa também fisicamente o comportamento do problema five spot em um reservatório de petróleo em um meio poroso heterogêneo.

Contudo, para se ter uma melhor base de comparação do modelo proposto na tese não basta apenas adaptar os dados fornecidos em uma malha fixa regular para uma geometria meshfree. Neste caso, é importante que os dados fornecidos pelo SPE 10 Model 2 sejam ajustados usando um histograma para representação das amostras do reservatório. Sendo assim, se faz necessário um tratamento mais rigoroso que gere todos os valores do campo de porosidade e de permeabilidade absoluta, no caso de uma geometria meshfree. 
No desenvolvimento deste trabalho foram utilizadas as funções em $G P U$ para acelerar a solução dos sistemas lineares. As funções em $G P U$ utilizadas são do tipo $A x=b$, implementados em MATLAB (Martin, 2010) e todos os modelos foram simulados usando um CPU Dell Precision win 64 com o processador $i 7$ 2960XM com 16.0 GB de memória com uma placa de vídeo nVidia 2000M GB GDDR3.

Por fim são apresentados as conclusões e os trabalhos futuros encontrado nessa pesquisa. 


\section{Conclusão e Trabalhos Futuros}

O objetivo deste trabalho foi fazer um estudo criterioso de modelos para a simulação de reservatórios de petróleo e buscar soluções práticas para simuladores numéricos de fluxo. A principal tentativa foi propor um método meshfree adaptativo de advecção para problemas de fluxo bifásico de fluidos incompressíveis e imiscíveis em meios porosos heterogêneos tridimensionais. Este método se baseia principalmente na combinação do método SemiLagrangeano adaptativo com interpolação local sem malha usando splines poliharmônicas como funções de base radial. O método proposto é uma melhoria e uma extensão do método adaptativo sem malha $A M M o C$ proposto por Iske e Käser (2005) para modelagem $2 D$ de reservatórios de petróleo. A presente tese inicialmente propõe um modelo em duas dimensões, contribuindo com uma melhoria significativa no cálculo do Laplaciano, utilizando os métodos sem malha de Hermite e Kansa. Depois, o método é ampliado para três dimensões (3D) e para um meio poroso heterogêneo. $\mathrm{O}$ método proposto é testado para problemas típicos de reservatórios, como o problema de five spot. Alguns resultados são comparados com os obtidos por sistemas bem conhecidos na indústria de petróleo.

Este estudo tomou como base os resultados de Barker e Thibeau (1997), Christie e Blunt (2001), Chen et al (2004) Hasle et al (2007) e Aarnes et al (2007). Ficou claro que a utilização de métodos meshfree é interessante para simulação de reservatórios. O trabalho de Iske e Käser (2005), que trata de meios porosos homogêneos, serviu como inspiração para o modelo proposto nesta pesquisa.

Esta pesquisa apresentou uma solução eficiente através da combinação do método de advecção Semi-Lagrangeano adaptativo com interpolação local usando splines poliharmônicas como funções de base radial. Para uma validação inicial desta solução foi feito um estudo comparativo unidimensional, com resultados quantitativos exatos, e um outro estudo, bi-dimensional, com grade regular, que apresentou um comportamento bastante satisfatório. 
Em seguida foi feito um estudo de um novo modelo bidimensional combinando os métodos meshfree com o método de Hermite, ou com o método de Kansa, e também com o método de advecção Semi-Lagrangeano adaptativo e interpolação local por splines poliharmônicas. Este estudo mostrou resultados compatíveis com a literatura.

Por fim, foi desenvolvida uma formulação para um novo método meshfree adaptativo de advecção para problemas de fluxo bifásico de fluidos incompressíveis e imiscíveis em meios porosos heterogêneos tridimensionais. Esta nova formulação permite simular reservatórios de meios porosos tanto homogêneos quanto heterogêneos. A porosidade e a permeabilidade absoluta do meio podem ser variáveis como também é possível a atualização do campo de pressão-velocidade e da saturação das fases do meio poroso de forma adaptativa.

Durante o desenvolvimento desta nova formulação foi feito um estudo do Software IMEX, como um experimento de sensibilidade dos processos envolvidos, para avaliar comportamentos e tendências entre as interfaces dos dois fluidos. Os resultados do $I M E X$ não puderam ser diretamente comparados com os obtidos pelo simulador desenvolvido porque a base de dados utilizada não tinha a mesma dimensão.

Ficou evidente que outros casos tridimensionais deveriam ter sido analisados utilizando dados de porosidade e permeabilidade absoluta mais próximos à realidade a fim de poder fazer uma melhor avaliação quantitativa do modelo proposto.

Este trabalho realizou um estudo crítico de trabalhos na área de simulação de reservatórios e esclareceu aspectos práticos para o desenvolvimento de simuladores numéricos de fluxo. A presente tese gerou um extenso material sobre a área e apresentou uma formulação completa que permite a simulação simples porém eficiente dos efeitos de forças viscosas, forças de capilaridade e forças gravitacionais. Esta é uma contribuição importante, visto que a literatura, apesar de ter muitos trabalhos didáticos e de pesquisa, carece de orientação sobre métodos meshfree. 


\section{Trabalhos Futuros}

Como mencionado anteriormente, há necessidade de estudar outros casos tridimensionais em meios porosos heterogêneos. Há dados reais disponíveis, inclusive o modelo SPE10 model 2 usado no exemplo do IMEX, Apêndice A, porém a extração dos mesmos para um ambiente meshfree é computacionalmente custosa.

O modelo proposto se mostrou bastante geral, ao considerar ambiente tridimensional, homogêneo ou heterogêneo, sujeito a efeitos diversos, porém não se preocupou com o tempo de processamento. Há a necessidade de se realizar um estudo no sentido de otimizar o modelo, inclusive com a adoção de novas rotinas em GPU. Não é possível paralelizar em relação ao tempo, porém a parte espacial (upstream points) pode ser feita em paralelo. Ainda nesta linha de melhorar o desempenho, recomenda-se a implementação do modelo numa linguagem mais eficiente e com estruturas de dados mais adequadas, visto que, na presente tese, o $M A T L A B$ foi usado apenas como prototipação.

Por fim, um estudo mais detalhado entre os métodos com malha e sem malha é recomendado como trabalho futuro.

No Apêndice A, é apresentado um caso real do problema five spot utilizando o simulador comercial IMEX (IMplicit-EXplicit Black-oil Simulator), conjuntamente com os aplicativos BUILDER e RESULTS GRAPH que fazem parte do mesmo ambiente computacional. 


\section{8 Referências bibliográficas}

Aguiar, T., V. Visualização de Fronteira entre Fluidos utilizando o Método SPH e o Algoritmo de Marching Cubes. Dissertação (Mestrado em Informática) Pontifícia Universidade Católica do Rio de Janeiro, 2009.

Alves, J. F. Uma análise comparativa dos principais métodos baseados em partículas para simulação de escoamentos. Dissertação (Mestrado em Informática) - Pontifícia Universidade Católica do Rio de Janeiro, 2008.

Akinci, N., Ihmsen, M., Akinci, G., Zurich, E., Teschner, M. Versatile RigidFluid Coupling for Incompressible SPH. ACM Transactions on Graphics (TOG) - Proceesings of ACM SIGGRAPH, New York, USA, Volume 31 Issue 4, July, Article No 62, 2012.

Aarnes, J. E., Gimse, T. Lie, K.-A. An introduction to the numeric of flow in porous media using Matlab. In G. Hasle, K.-A. Lie, and E. Quak, editors, Geometrical Modeling, Numerical Simulation and Optimisation: Industrial Mathematics at SINTEF, pages 265\{306. Springer Verlag, Berling Heidelberg New York, 2007.

Atluri, S. N., and Zhu, T. A new meshless local Petrov-Galerkin (MLPG) approach in computational mechanics. Comput. Mech. 22: 117-127, 1998.

Atluri, S. N., Cho, J. Y, Kim, H. G. Analysis of thin beams, using the meshless local new meshless local Petrov-Galerkin (MLPG) method, with generalized moving least squares interpolation. Computational mechanics, 24: 334-347, 1999.

Atluri, S. N., and Shen, S. The Meshless Local Petrov-Galerkin (MLPG) Method. Tech Science Press, Encino, CA, 2002.

Atluri, S. N., and Shen, S. The meshless local Petrov-Galerkin (MLPG) method: A simple \& lesscostly alternative to the finite element and boundary element methods. CMES:Computer Modeling in Engineering \& Sciences, vol. 3, no. 1, pp. 11-52, 2002.

Atluri, S. N. The Meshless Methods (MLPG) For Domain \& BIE Discretizations, Tech Science Press (Encino, CA), 2004.

Aziz, K., and Settari, A. Petroleum reservoir simulation. Elsevier, London and New York, 1979.

Buckley, S. E., and Leverett, M. C. Mechanism of displacement in sand. Trans.AIME, 146, 187 - 196, 1942.

Baraff, D., Witkin, A., Kass, M., Anderson J. Physically Based Modeling (a Little Fluid Dynamics for Graphics), ACM SIGGRAPH Course Notes. ACM, 2003.

Barker, J. W., and Thibeau, S. A critical review of the use of pseudorelative permeabilities for upscaling. SPE Reservoir Eng., 12(2):138ir Eng. Ups, 1997. 
Batchelor, G. K. An introduction to fluid dynamics. Cambridge University Press, 1967.

Behrens, J., Iske, A., Käser, M. Adaptive Meshfree Method of Backward Characteristics for Nonlinear Transport Equations, in Meshfree Methods for Partial Differential Equation, Griebel M. and M. A. Schweitzer M. A. (eds.), Springer-Verlag, Heidelberg, 21-36, 2002.

Belystschko, T., Lu, Y. Y., Gu, L. Element Free Galerkin Methods. International Journal for Numerical Method in Engineering, 37,229-256, 1994a.

Belystschko, T., Lu, Y. Y., Gu, L. A New Implementation of the Element, Free Galerkin Method. Computer Methods in Applied Mechanics and Engineering, 113, 397-414, 1994b.

Belystschko, T., LU, Y. Y., GU, L. Fracture and Crack Growth by EFG Methods. Modeling Simul. Muter. Sci. Eng., 2,519-534, 1994c.

Belystschko, T., KRONGAUZ, Y., ORGAN, O., FLEMING, M., KRYSL, P. Meshless methods: an overview and recent developments, Comp. Meth. Appl. Mech. Eng. 139, pp. 3-47. 1996.

Bird, R. B., Stewart, W. E., Lightfoot, E. N. Transport Phenomena (Revised Second Edition ed.). John Wiley \& Sons. p. 83. ISBN 978-0-470-11539-8, 2007.

Breitkopf, P., Touzot, G., Villon, P. Double grid diffuse collocation method. Computational Mechanics, vol. 25, no. 2/3, pp. 199-206, 2000.

Brooks, A. N., Corey, A. T. Hydraulic Properties of Porous Media. Colorado State University, 1964.

Brown, S. and Collier R., 2006. Flooding Ice Age: The Meltdown Using Wavesynth and Point-Based Froth, ACM SIGGRAPH Sketches and Applications. ACM Press.

Buhmann, M. D. Radial Basis Functions: Theory and Implementations, Cambridge University Press (Cambridge), 2003.

Chen, Z., Huan, G.; Li, B. An Improved IMPES Method for Two-Phase in Porous Media. Transport in Porous Media, 54 (3):361-376. ISSN 0169-3913, March, U.S.A, 2004.

Chen, Z., Huan, G., Ma, Y. Computational Methods for Multiphase Flows in Porous Media. Computational Science and Engineering. Society for Industrial and Applied Mathematics, 2006.

Cheney, E. W., Light, W. A. A Course in Approximation Theory, Brooks/Cole (Pacific Grove, CA), 1999.

Chentanez, N., Muller, M., Kim, Y. T. Coupling 3D Eulerian, Heightfield and Particle Methods for Interactive Simulation of Large Scale Liquid Phenomena, in Proceedings of ACM SIGGRAPH/EUROGRAPHICS Symposium on Computer Animation (SCA), Copenhagen, July 21-23, 2014.

Chavent, G., Jaffré, J. Mathematical Models and Finite Elements for Reservoir Simulation: Single Phase, Multiphase and Multicomponent Flows through Porous Media. Studies in Mathematics and its applications. North-Holland, 1986. 
CMG - COMPUTER MODELLING GROUP LTD. Imex.10 User's Guide, Manual de Referência, 2007.

Corey, A. T. The Interrelation Between Gas and Oil Relative Permeabilities, Producer's Monthly 19, No. 1, 38-41, 1954.

Courant, R., Friedrichs, K., Lewy, H. On the partial difference equations of mathematical phsysics. AEC Research and Development Report, NYO-7689, New York: AEC Computing and Applied Mathematics Centre - Courant Institute of Mathematical Sciences, pp V + 76, archived from the original on October 23, 2008: translated from the German by Phyllis Fox. This is an earlier version of the paper Courant, Friedrichs \& Lewy 1967, circulated as a research report, 1928.

Christie, M. A., Blunt, M. J. Tenth SPE comparative solution project: A comparison of upscaling techniques. SPE Reservoir Eval. Eng., 4(4):308 oir . url: www.spe.org/csp, 2001.

Darcy, H. Détermination des lois d'écoulement de l'eau ‘a travers le sable. In Les Fontaines Publiques de la Ville de Dijon, pages 590-594. Victor Dalmont, Paris, 1856.

De Forest, E. L. Additions to a Memoir on Method of Interpolation Applicable to the Graduation of Irregular Series. Annual Report of the Board of Regent of the Smithsonian Intitution for 1873, pp 319-353, 1874.

Deller, J. The handbook of groundwater engineering, CRC Press, 2006.

Desbrun, M., Gascuel, M. P. Smoothed particles: a new paradigm for animating highly deformable bodies. In Computer Animation and Simulation 96 (Proceedings of EG Workshop on Animation and Simulation), p. 61-76, 1996.

Devadoss, L. S., Rourke O. J. Discrete and Computational Geometry. New jersey, USA, 2011.

Douglas Jr , J., Russel; T. F. Numerical methods for convection-dominated diffusion problems based on combining the method of characteristics with finite element or finite difference procedures, SIAM J. Numer.Anal. 871-885, 19, 1982.

Duarte, C. A., Oden, J. T. Hp Clouds-an hp Meshless Method. Numerical Methods for Partial Differential Equations, v12, 673-705, 1996.

Durran, D. R. Numerical Methods for Waves Equations in Geophysical Fluid Dynamics . 1 ed. New York, Springer-Verlag, 1998.

Ewinh, R. E. The mathematics of reservoir simulation. SIAM, 1983.

ENGLISH, E., QIU, L., FEDKIW, R. Chimera Grids for Water Simulation. ACM SIGGRAPH Symposium on Computer Animation, 2013.

Enright, D., Fedkiw, R., Ferziger, J., and Michell, I. A Hybrid Particle Level Set Method for Improved Interface Capturing, Journal of Computational Physics, 183, 83-116, 2002.

Ertekin, T., Abou-kassem, J. H., King, G. R., Basic Applied Reservoir simulation. SPE Texbook Series. Vol. 7, p. 107, p.181, 2001. 
Fasshauer, G. E. Meshfree Approximation Method with Matlab. 6a. Ed: World Scientific Publishing Co, 2007.

Firoozjaee, A., Hendi, E. Farvizi, F. Element Free Gelerkin Method for 2-D Potential Problems. Applied Mathematics, 6, 149-162: 10.4236/am .61015, 2015.

Forrester, A. I. J., Sóbester, A., Keane, A. J. Engineering Design via Surrogate Modelling: A Practical Guide, Chichester, 240 pages, ISBN 978-0-470-06068-1, Southampton City, United Kindom, 2008.

Fox, R. W., MC Donald A. T. Introdução a Mecânica dos Fluidos, 5 Ed., LTC, Livros Técnicos e Científicos S. A., Rio de Janeiro, 2001.

Franke, C., Schaback, R. Solving partial differential equations by collocation using radial basis functions, Appl. Math. Comp. 93, pp. 73-82, 1998a.

Franke, C., Schaback, R. Convergence orders of meshless collocation methods using radial basis functions, Adv. in Comput. Math. 8, pp. 381-399, 1998b.

Geiger, W., Leo, M., Rasmussen, N., Losasso, F., and Fedkiw, R. So Real It'll Make You Wet, ACM SIGGRAPH Sketches and Applications. ACM Press, 2006.

Gjennestad, A., Munkejord, S.T. Modelling of Heat Transport in Two-Phase Flow and of Mass. SINTEF, Rondheim, Norway, 2015.

Gingold, R., A., Monaghan, J., J. Smoothed Particle Hydrodynamics: Theory and Application to Non-spherical Star. Monthly Notices of de Royal Astronomical Society. v.181, p. 375-389, 1997.

Goldstein, H., Poole C., Safko J. Classical Mechanics, - Addison-Wesley, 3Ed, 2002.

$\mathrm{Gu}, \mathrm{C}$., Wahba, G. Semiparametric analysis of variance with tensor product thin plate splines, J. Roy. Statist. Soc. Ser. B 55, pp. 353-368, 1993.

Aitken, Z. H.; Fan, H., Ei-Awady, J. A., Greer, J. R. The effect of size, orientation and alloying on the deformation of AZ31 nanopillars. Journal of Mechanics and Physics of Solids. 76, 208-223, 2015.

Gu, W., Jafary-Zadeh, M., Chen, D. Z., Wu, Z., Zhang, Y-W.; Srolovitz, D. J.; Greer, J. R. Mechanisms of Failure in Nanoscale Metallic Glass. Nano Letters 14, 5858-5864, 2014.

Guedelman, E., Selle, A., Losasso, F., and Fedkiw, R. Coupling Water and Smoke to Thin Deformable and Rigid Shells, ACM SIGGRAPH Sketches and Applications. ACM TOG 24, Pages 973-981, 2005.

Gram, J. P. Uber Entwicklung Reeler Functionen in Reiher Mittelst der Method der Kleinsten Quadrate, J. Math, 1883.

Griebel, M., Schwetzer, M.A. Meshfree Methods for Partial Differential Equations VII Lecture Notes in Computational Science and Engineering, Hardcover, California, Unite States, December 3, 2014.

Hasle, G., Lie, K.-A, Quak, E. Cook, R.; Geometrical Modeling Simulation and Optimisation: Applied Mathematics at SINTEF. $1^{\text {a }}$. ed. Springer Verlag Berling Heidelberg New York, 2007.

Havas, P. The Range of Application of the Langrange Formalism-I. Nuovo Cimento Suppl. 5, 363, 1957. 
Iversen, J., Sakaguchi, R. Growing Up with Fluid Simulation on: The Day After Tomorrow, ACM SIGGRAPH Sketches and Applications. ACM Press.a, 2004.

Irving, G., Guendelman, E., Losasso, F., and Fedkiw, R. Efficient Simulation of Large bodies of Water by Coupling Two and Three Dimensional Techniques, ACM SIGGRAPH Sketches and Applications, 2006.

Iske, A. Multiresolution Methods in Scattered Data Modelling, Lecture Notes in Computational Science and Engineering 37, Springer Verlag Berlin, 2004.

Iske, A., and Käser, M. Two-Phase Flow Simulation by AMMoC, Method of Characteristics, Computer Modeling in Engineering \& Sciences (CMES) 7(2), 2005, 133-148, 2005.

Iske, A. On the approximation order and numerical stability of local Lagrange interpolation by polyhamonic splines. Modern Developments in Multivariete Approximation, W. Haussmann, K. Jetter, M. Reimer, J. Stockler (eds.), ISNM 145, Birkhäuser, Basel, 153-165, 2003.

Kalnay E., Atmospheric Modeling, Data Assimilation and Predictability, Cambridge University Press, Cambridge, 2003.

Kansa, E. J. Multiquadrics - A scattered data approximation scheme with applications to computational fluid-dynamics - II: Solutions to parabolic, hyperbolic and elliptic partial differential equations, Comput. Math. Appl. 19, pp. 147-161, $1990 \mathrm{~b}$.

Krishnamurti, T. N., Numerical Integration of Primitive Equations by a quasiLagrangian Advective Scheme, Journal of Applied Meteorology, vol 1, pp 508$521,1962$.

Krysl, P., Belytschko, T. Analysis of thin plates by element-free Galerkin method. Comput. Mech. 17: 26-35 Krysl P, Belytschko T 1996b Analysis of thin shells by element-free Galerkin method. Int. J. Solids Struct. 33: 3057-3080, 1996a.

Larsson, E., Fornberg, B. A numerical study of some radial basis function based solution methods for elliptic PDEs, Comput. Math. Appl. 46, pp. 891-902, 2003.

Lemos, N. Mecânica Analítica. Tese (Doutorado em Física) - Departamento de Física - Universidade Federal Fluminense. 2002.

Lewiner, T., Lopes, H., Vieira, A. W., Tavares, G. Efficient implementation of Marching Cubes cases with topological guarantees. Journal of Graphics Tools, 8 (2), 1-15, 2003.

Lentine, M., Cong, M., Patkar S., Fedkiw, R. Simulating Free Surface Flow with Very Large Time Steps. ACM SIGGRAPH Symposium on Computer Animation, 2012.

Li, S., Liu, W. K. Meshfree and particle methods and their applications. Applied Mechanics Review, vol. 55, no. 1, pp. 1-34, 2002.

Lin, H., Atluri, S. N. Analysis of incompressible Navier-Stokes flows by the meshless MLPG method. Computer Modelling in Engineering \& Sciences, 2(2), 117-142, 2001. 
Liszka, J., Orikisz. The Finite Difference Method for Arbitrary Irregular mesh A Variational Approach to Applied Problems, $2^{\text {nd }}$ In Congress on Num. Meth. Eng. Paris, pp 277- 288, 1980.

Liu, G. R., Gu, Y. T. Coupling of element-free Galerkin method and hybrid boundary element methods using modified variational formulation. Comput. Mech. 26: 166-173, 2000.

Liu, G. R., Liu, M., B. Smoothed Particle Hydrodynamics: a meshfree particle method. World Scientific Publishing, 2003.

Liu, G. R. Mesh free methods-moving beyond the finite element method. CRC Press, United States, October 6, 2009.

Losasso, F., Gibou, F., Fedkiw, R. Simulating water and smoke with an octree data structure. ACM Trans. Graph. (SIGGRAPH Proc.), 457-462, 2004.

Losasso, F, Shinar, T., Selle, A., and Fedkiw, R. Multiple Interacting Liquids, ACM SIGGRAPH Sketches and Applications. ACM TOG 25, 2006.

Losasso, F, Shinar T., Talton, J., Kwatra, N., and Fedkiw, R. Two-way Coupled SPH and Particle Level Set Fluid Simulation. IEEE TVCG 14, 2008.

Lucy L. B. A numerical approach to the testing of the fission hypothesis. The Astronomical Journal, 82: p. 1013-1024, 1977.

Marshall, F. Numeric Solution of Philip's Redistribution Problem. Diploma Thesis, Germany, 2009.

Martin, J. Accelerating MATLAB with NVIDIA GPUs. Principle Architect, MathWorks Parallel Computing Products, Reino Unido, 2010.

Mesinger, F., Arakawa, A. Numerical methods used in atmospheric models, volume I, In: Garp Publication Series No. 17, WMO/ICSU Joint Organazing Committee, 1976.

Miller, G. Pearce, A. Globular Dynamics: A Connected Particle System for Animating Viscous Fluids. Computers and Graphics, 13 (3), 305-309, 1989.

Monaghan, J. J. Why particle methods work. SIAM Journal of Scientific and Statistical computing, 3 (4), 422-433, 1982.

Monaghan, J., J. Smoothed Particle Hydrodynamics. Ann. Rev. Astron. Astrophysics, 30, 543-574, 1992.

Monaghan, J., J. Simulating free surface flow with SPH. Journal of Computational Physics, 110: p. 399-406, 1994.

Muller, M., Charypar, D., Gross, M. Particle based fluid simulation for interactive applications. Proceedings of ACM SIGGRAPH Symposium on Computer Animation, 154-159, 2003.

Muller, M., Solenthaler, B., Keiser, R., Gross, M. Particle based Fluid-Fluid Interaction. Eurographics ACM Symposium on Computer Animation, 2005.

Nakamura, F. I. Animação Interativa de Fluido baseada em Partículas pelo Método SPH. Dissertação (Mestrado em Informática) - Pontifícia Universidade Católica do Rio de Janeiro, 2007. 
Napolitano, L. Implementação Numérica do Método Level Set para propagação de curvas e superfícies. Dissertação (Departamento de física e Ciências dos Materiais) - Instituto de física de São Carlos - Universidade São Paulo, 2004.

Nayroles, B., Touzot, G., Villon, P. Generalizing the finite element method: diffuse approximation and diffuse elements. Comput. Mech. 10: 307-318, 1992.

Osher, S., Sethian, J. Fronts Propagating with Curvature Dependent Speed: Algorithms Based on Hamilton - Jacobi Formulations, J. Comput. Phys. 79, 1249, 1988.

Osher, S., Fedkiw, R. Level Set Methods and Dynamic Implicit Surfaces. Number 153 in Applied Mathematical Sciences. Springer, 6, 8, 29, 34, 73, 2003.

Patkar, S., Karpman, D., Fedkiw, R. A Hybrid Lagrangian-Eulerian Formulation for Bubble Generation and Dynamics. Anaheim, California, 2013.

Peaceman, D. W. Fundamentals of Numerical Reservoir Simulation. Elsevier, Amsterdam, 1977.

Preparata, F. P., Shamos, I. M. Computational Geometry an Introduction. New York, USA, 1985.

Reeves, W., T. Particle systems - a technique for modeling a class of fuzzy objects. In Proc. ACM SIGGRAPH 83, 359-376, 1983.

Robert, A., Henderson, J., Turbull, C. An Implicit Time Integration Scheme for Baroclinic Models of the Atmosphere, Monthly Weather Review, vol. 100, pp. 329-335, 1972.

Robert, A. A Stable Numerical Integration Scheme for the Primitive Meteorological Equations, Atmosphere-Ocean, vol. 19, pp. 35-46, 1981.

Rosa, A. J., Carvalho, R. S.; Xavier, J. A. Engenharia de reservatórios de petróleo - Rio de Janeiro: Interciência: PETROBRAS, 2006.

Sawyer, J. S. A Semi-Lagrangian method of Solving the Vorticity Advection Equation, Tellus, vol. 15, pp. 336-342, 1963.

Schaback, R. Limit problems for interpolation by analytic radial basis functions, J. Comp. Appl. Math., to appear, 2006b.

Sethian, J. Level Set Methods and Fast Marching Method, Volume 3 of Cambridge Monographs on Applied computational Mathematics. Cambridge, 1999. 6, 8, 29, 1999.

Staniforth, A., Côté, J. Semi-Lagrangian integration schemes for atmospheric models - A review, Monthly Weather Review, vol. 119, pp. 2206-2223, 1991.

Stomakhin, A., Schroeder, C., Jiang, C., Chai, L., Teran, J., Selle, A. Augmented MPM for phase-change and varied materials. ACM Transactions on Graphics (TOG) - Proceedings of ACM SIGGRAPH. Volume 33 Issue 4, July, Article No. 138, New Yok, USA, 2014

Terzopoulos, D., Platt, J., and Fleischer, K. Heating and Melting Deformable Models (from Goop to Glop). In Proc. Eighth Graphics Interface Conf., 219-226, 1989.

Thomas, J. E. Fundamentos de Engenharia de Petróleo. 2. ed., Editora Interciência p. 200-201, 2001. 
Thornton, J. Directable Simulation of Stylized Water-Splash Effects in 3D Space, ACM SIGGRAPH Sketches and Applications. ACM Press, 2006.

Van Genuchten, M. T. A Closed-Form Equation for Predicting the Hydraulic Conductivity of Unsaturated Soils. American Journal of Soil Science, 44:892898, 1980.

Vargas, M. Introdução à Mecânica dos Solos - Mc. Gran-Hill do Brasil, 1977.

Vorgelegt, V. The Level Set Method for Capturing interfaces with applications in Two Phase Flow Problems. Diplom-Mathematikerin Eva Loch. Dissertation, 2013.

Zhu, A., Xu, Q., Jiang, Z. Characteristics Weak Galerkin Finite Element Methods for Convection-Dominated Diffusion Problems. School of Mathematical Sciences, Shandong Normal University, Accepted 12 May 2014; Published 27 May, China, 2014.

Welander, P. Studies on the General Development of Motion in a Twodimensional, Ideal Fluid. Tellus, vol. 7, pp. 141-156, 1995.

Wendlan, H. Scattered Data Approximation. Cambridge University Press, 2005.

Wiin - Nielsen, A. On the Application of Trajectory Methods in Numerical Forecasting. Tellus, vol. 11, pp. 180-196, 1959.

$\mathrm{Wu}, \mathrm{C}$., Liu, W. V., Fradkin, E. Competing orders in coupled Luttinger liquids. Phys. Rev. B 68, 115104, 2003. 


\section{9 Apêndices}

\section{1. Apêndice $A$}

\section{Modelo de five spot real usando o IMEX}

Com o intuito de validar o modelo proposto de advecção bifásico imiscível e incompressível utilizando um meio poroso heterogêneo, desenvolvido na tese, optou-se por comparar com uma solução de referência baseado num software comercial de simulação de reservatórios. Para isto, foi feito o mesmo problema de five spot, utilizando o simulador comercial IMEX (IMplicit-EXplicit Black-oil Simulator), conjuntamente com os aplicativos BUILDER e RESULTS GRAPH que fazem parte do mesmo ambiente computacional. O IMEX é um simulador de reservatórios da empresa Canadense Computer Modeling Group Ltd. (CMG, 2007), cuja formulação numérica baseia-se no método de diferenças finitas. Ele trabalha com simulações bifásicas (óleo-água, óleo-gás ou ainda gás-água) ou trifásicas (óleo, água e gás) podendo usar coordenadas cartesianas e cilíndricas em ambientes bidimensionais e tridimensionais (2D e 3D) (Ertekin et al, 2001).

A comunicação neste simulador com o usuário é dado por meio de um arquivo de entrada e saída de dados. O arquivo de entrada (.dat) contém as seguintes informações :

I) Controle de Entrada e Saída de dados; Início do arquivo.

II) Descrição do Reservatório; Começa com a palavra-chave *GRID.

III) Propriedades dos Componentes; Começa com a palavra-chave *MODEL.

IV) Propriedades Rocha-Fluido; Começa com a palavra-chave *ROCKFLUID.

V) Condições Iniciais; Começa com a palavra-chave *INICIAL.

VI) Controle dos Métodos Numéricos; Começa com a palavra-chave *NUMERICAL. 
VII) Dados de Poço e Dados Recorrentes; Começa com a palavra-chave $* R U N$ e termina com a palavra-chave ${ }^{*} S T O P$.

Importante pontuar que o controle de entrada e saída de dados determina como o simulador imprimirá os resultados da rodada, bem como as informações que deverão ser incluídas no modelo. Nesta parte é de extrema importância a definição do sistema de unidades a ser utilizado pelo simulador. Usando a palavra chave *INUNIT especificam-se as unidades de entrada de dados, enquanto *OUTUNIT especifica as unidades de saída de saída de dados, (CMG, 2007). O sistema de unidades utilizado nesta tese é o sistema internacional, SI.

É necessário fornecer as dimensões do reservatório, o numero de células em cada uma das três dimensões e o espaçamento entre elas. Os poços são posicionados de forma verticais e no caso do poço posicionado na vertical é necessário definir sua localização no reservatório, ou seja, sua posição $i, j, k$ dentro do reservatório e sua função (injetor ou produtor dependendo do caso). $\mathrm{O}$ seu sentido de crescimento é sempre na direção $k$.

Existem quatro tipos de malhas que podem ser utilizadas no $C M G$, a saber: cartesiano, regular, profundidade e espessura variáveis. Foi utilizado o modelo original SPE Model 2 (3D) do SPE66599 (Christie e Blunt, 2001), que usa uma malha regular.

O aplicativo BUILDER é usado para determinar a geometria e a malha do reservatório. Ao simular o modelo (.dat) no IMEX, todos os resultados numéricos são armazenados em arquivos de extensão .IRF e.$M R F$, enquanto o aplicativo RESULT REPORT, permite a escolha de apenas os dados necessários de acordo com a aplicação. Esses dados serão avaliados no aplicativo RESULTS GRAPH para fins de visualização de todas as grandezas como a pressão e a saturação (CMG, 2007). A seguir serão apresentados alguns resultados utilizando o simulador IMEX.

\section{Simulação do Problema real de five-spot utilizando o IMEX}

No problema em questão foi simulado um caso tridimensional de recuperação de óleo através da injeção de água, avaliando a saturação com relação ao avanço ao longo do tempo. Como foi dito anteriormente o problema five-spot é um problema bifásico que consiste em injetar, neste caso, o fluido água do 
centro do reservatório através do raio da injeção e o fluido óleo é empurrado pela saturação da água ao longo do tempo, para os quatros outros poços de produção.

A seguir serão ilustrados os resultados usando o simulador comercial IMEX. Da Figura 70 à Figura 72 são exibidas a geometria do reservatório e a porosidade do meio heterogêneo, gerados a partir do próprio aplicativo BUILDER no ambiente do simulador IMEX. Na Figura 71 e na Figura 72 é possível observar a formação "Tabert" e a formação "Upper Ness".

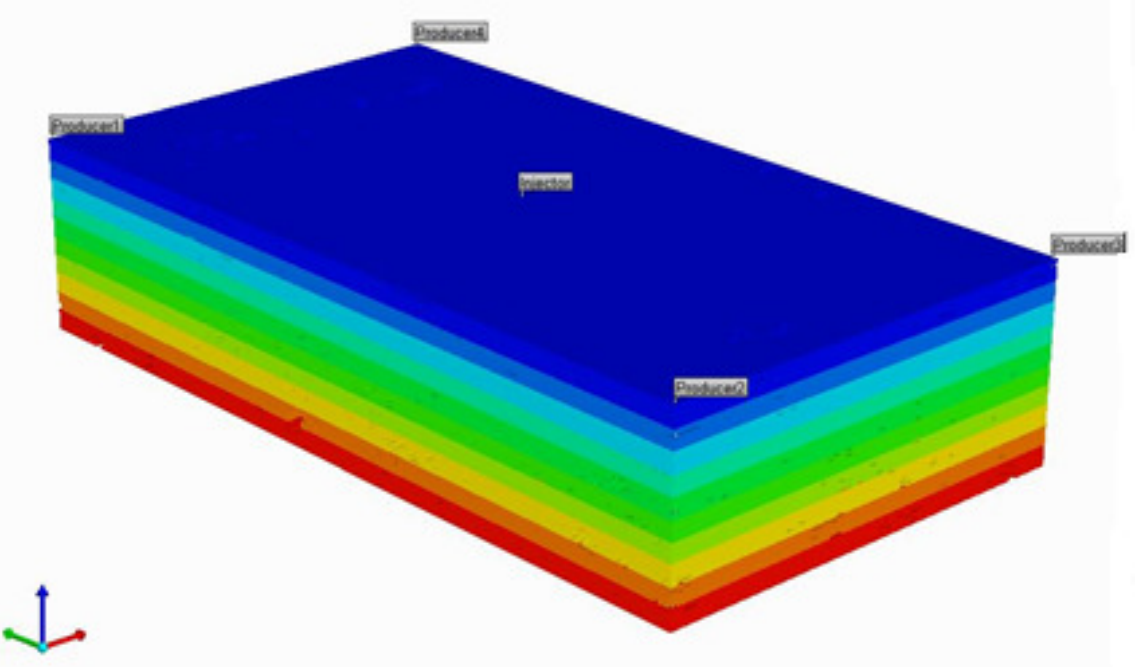

Figura 70: Geometria do modelo SPE 10 -Model 2. 


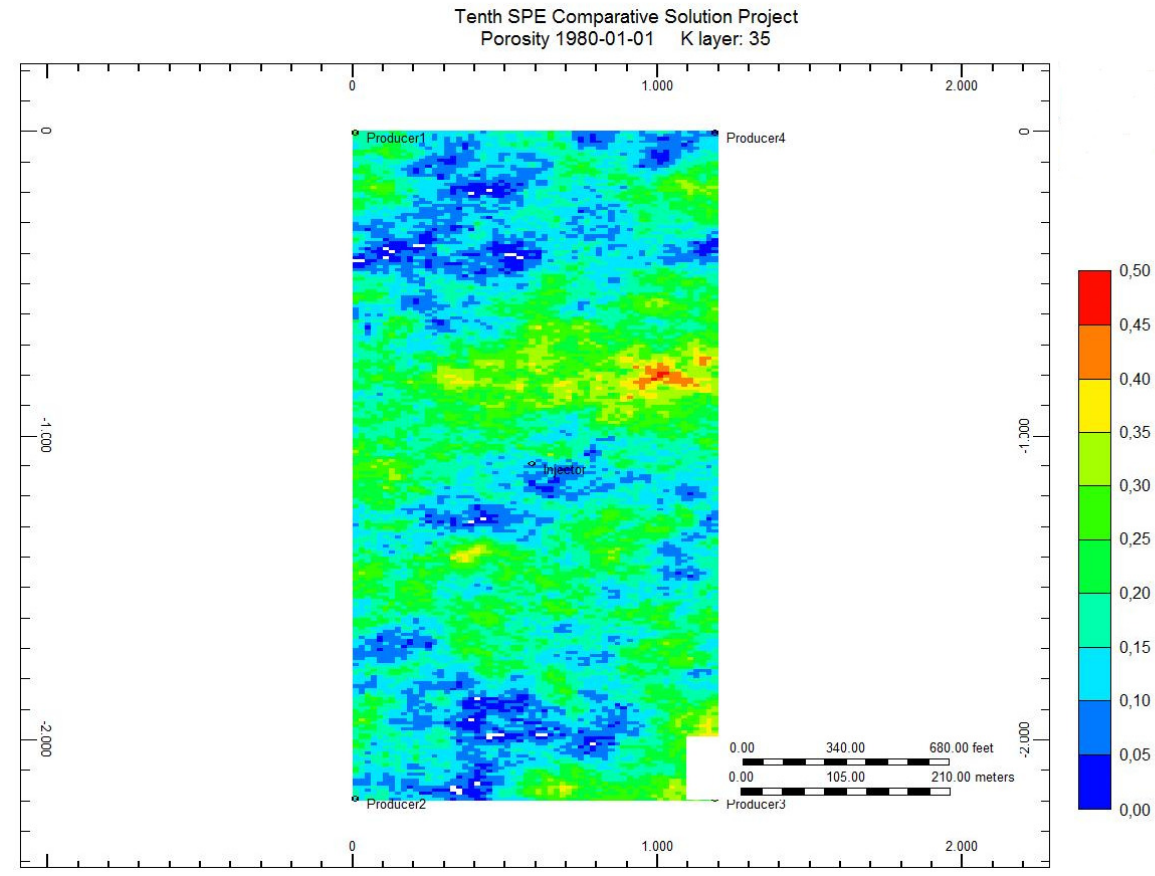

Figura 71: Porosidade do meio heterogêneo (Formação “Tabert”).

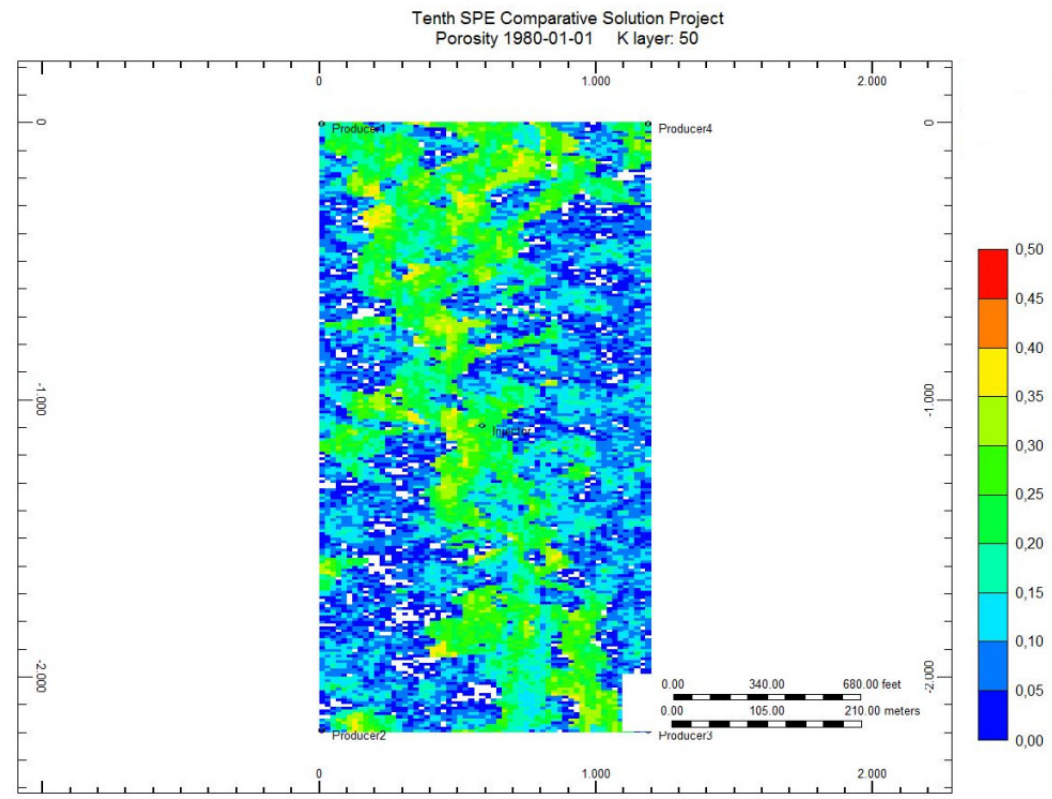

Figura 72: Porosidade do meio heterogêneo (Formação “Upper Ness”).

Enquanto que da Figura 73 à Figura 75 mostrem uma queda de pressão no reservatório durante um período de cinco anos. 


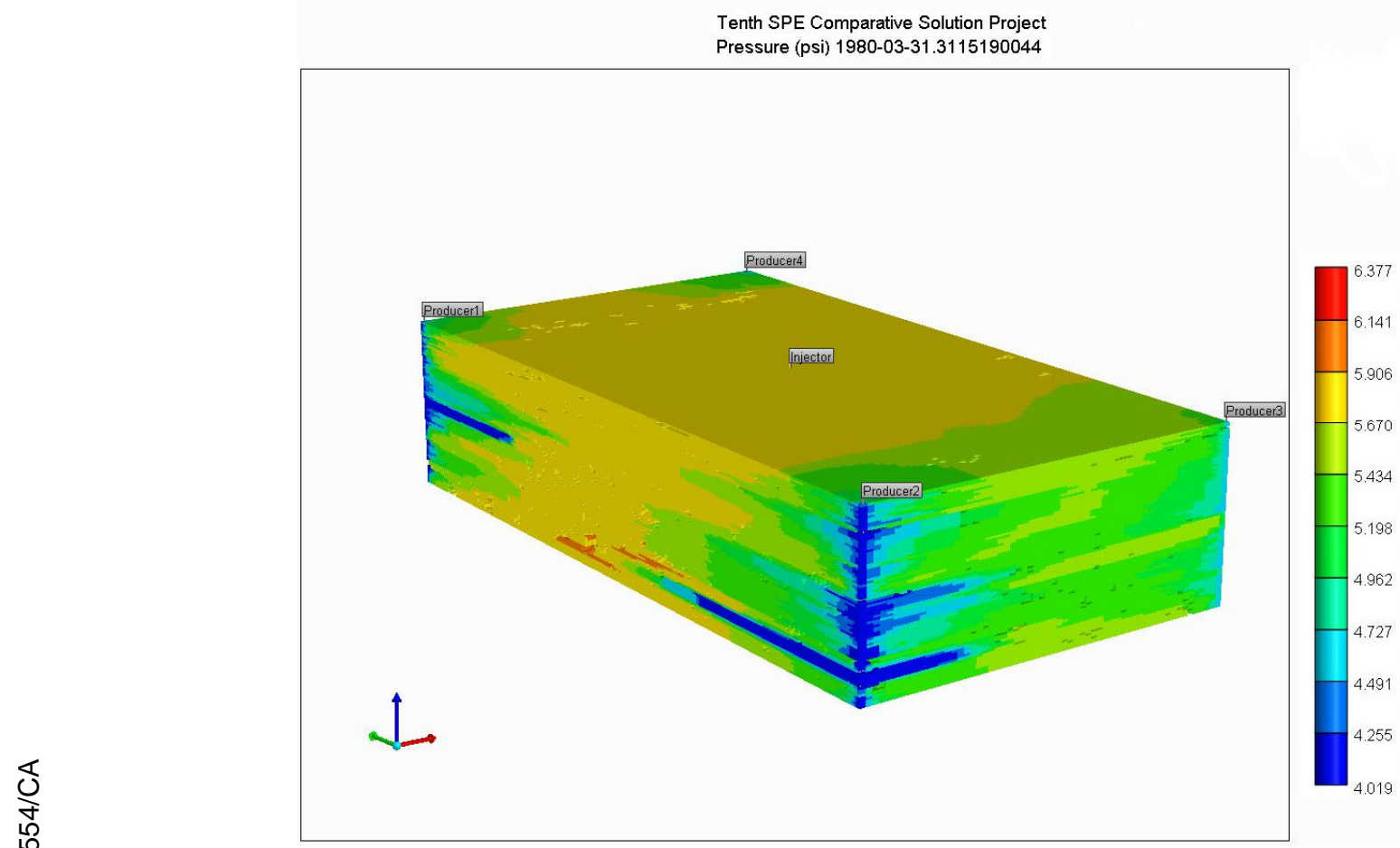

Figura 73: Variação de pressão no reservatório (Aproximadamente três meses depois)

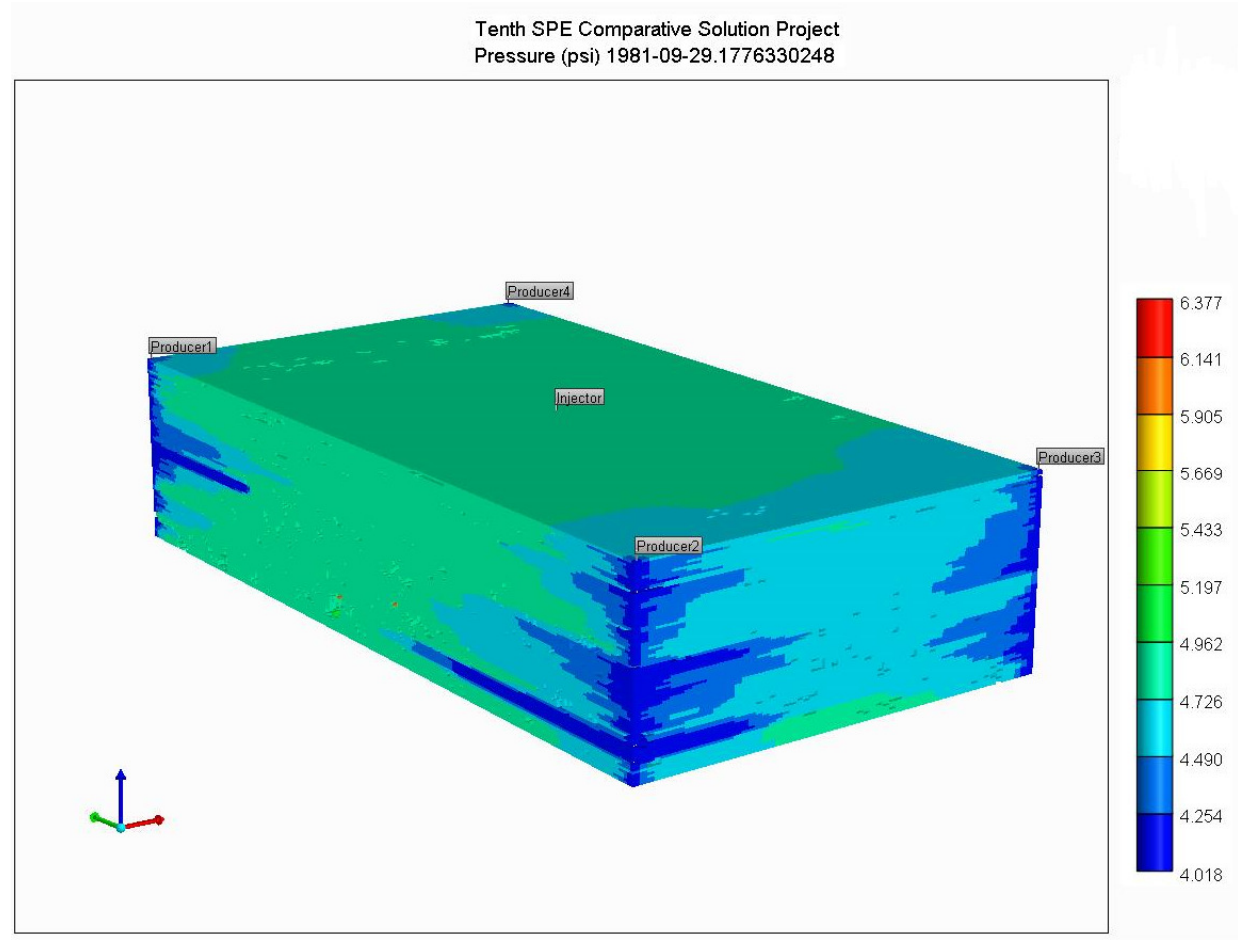

Figura 74: Variação de pressão no reservatório (Aproximadamente um ano e nove meses depois) 


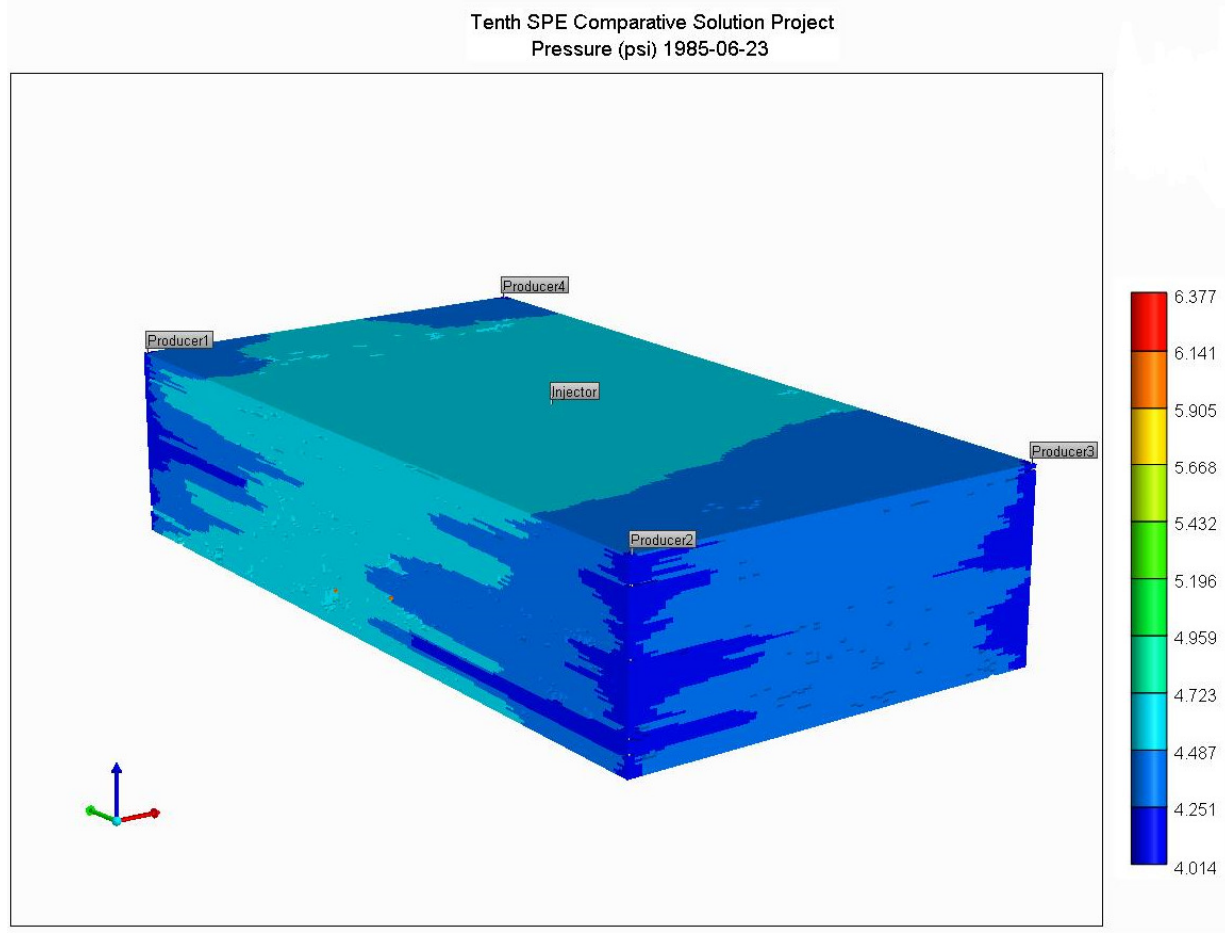

Figura 75: Variação de pressão no reservatório (Aproximadamente cinco anos e seis meses depois)

Finalmente são apresentadas a seguir as variações do campo de saturação no reservatório. Portanto, da Figura 76 a Figura 80 são ilustradas os campos de saturações do meio poroso heterogêneo para diferentes camadas e também durante um período aproximadamente de cinco anos e seis meses. Essas figuras foram obtidas também pelo aplicativo IMEX e RESULT. 


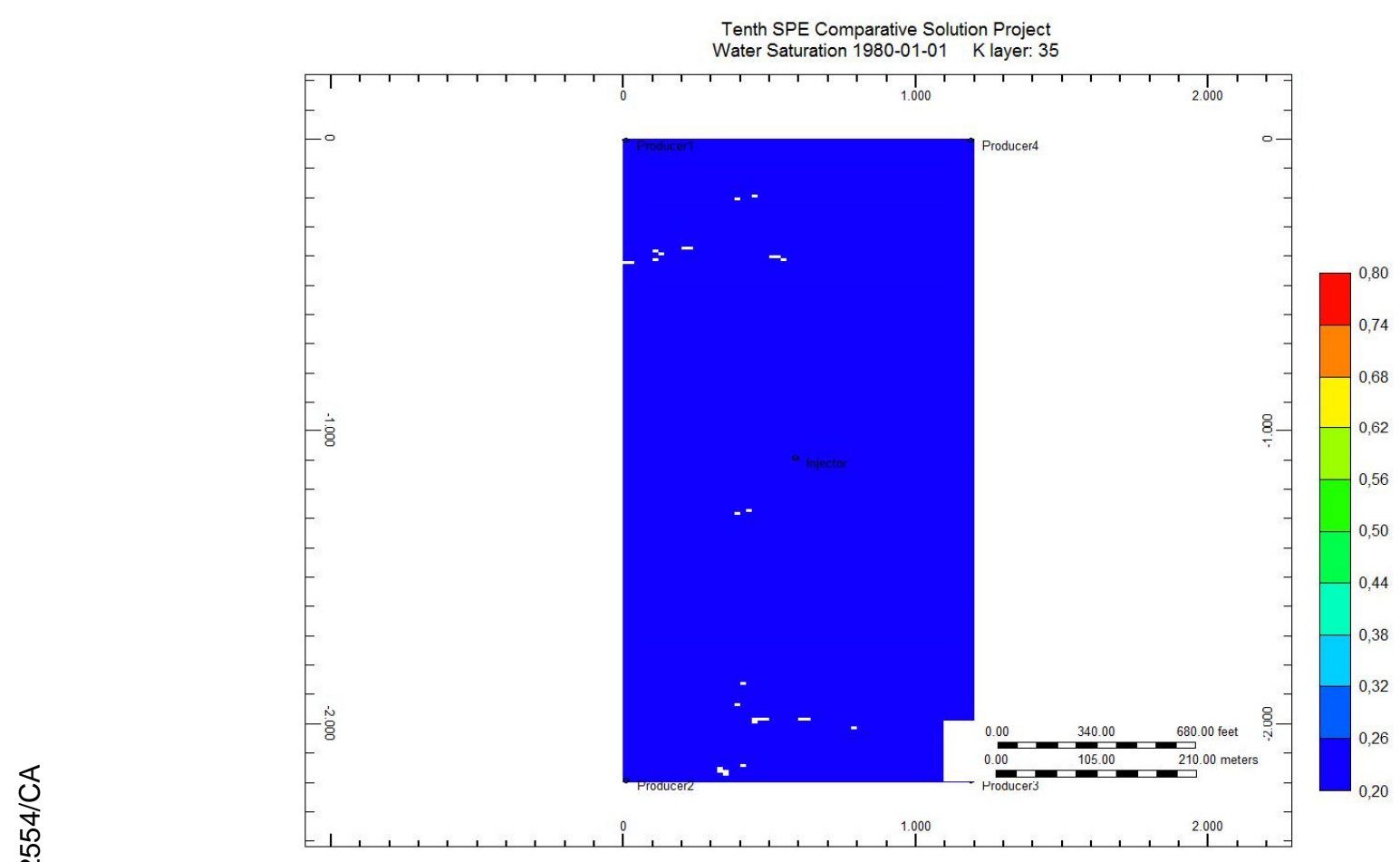

Figura 76: Saturação da água no meio heterogêneo no layer 35

Tenth SPE Comparative Solution Project Water Saturation 1980-03-31.3115190044 K layer: 35

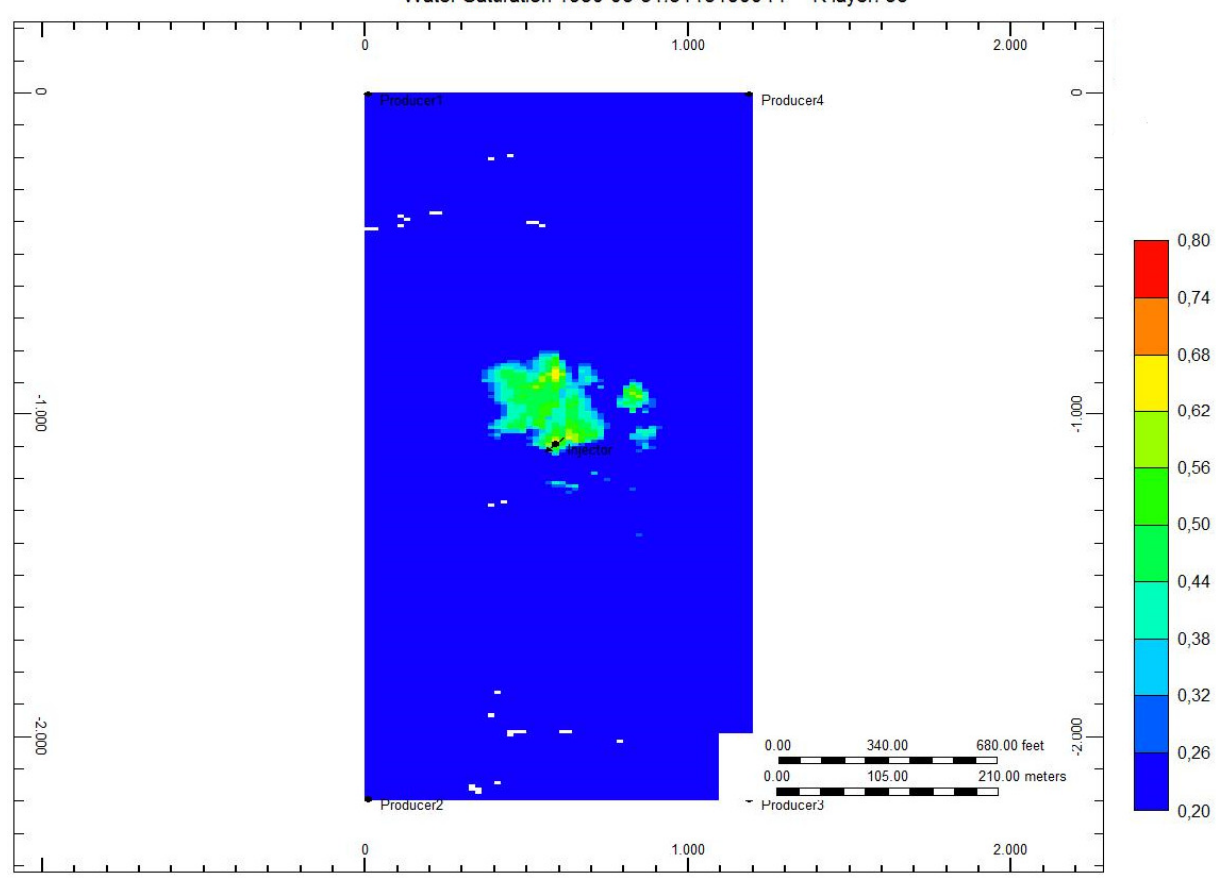

Figura 77: Saturação da água no meio heterogêneo do layer 35 (Três meses depois, aproximadamente) 


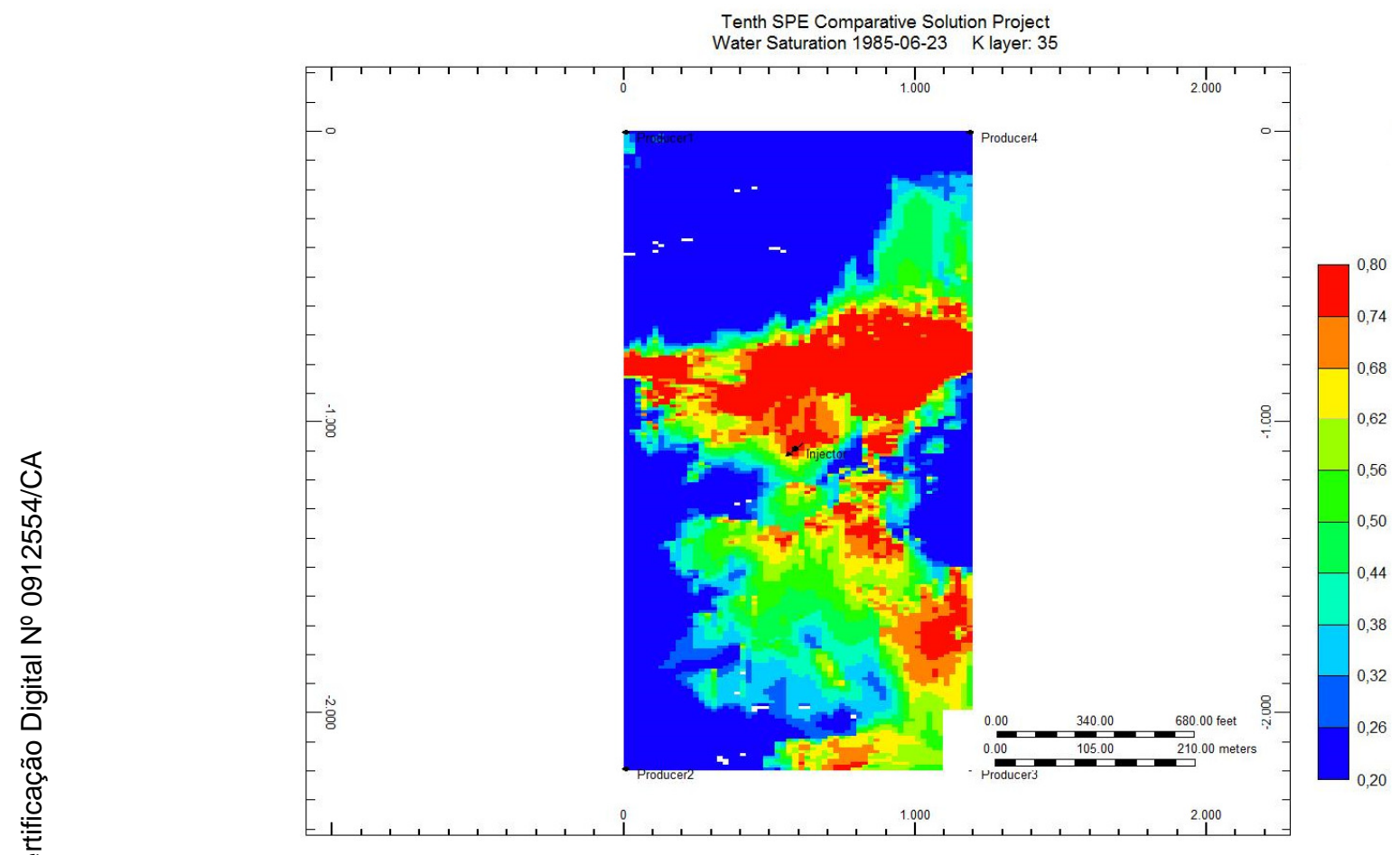

Figura 78: Saturação da água no meio heterogêneo do layer 35 (Cinco anos depois, aproximadamente) 


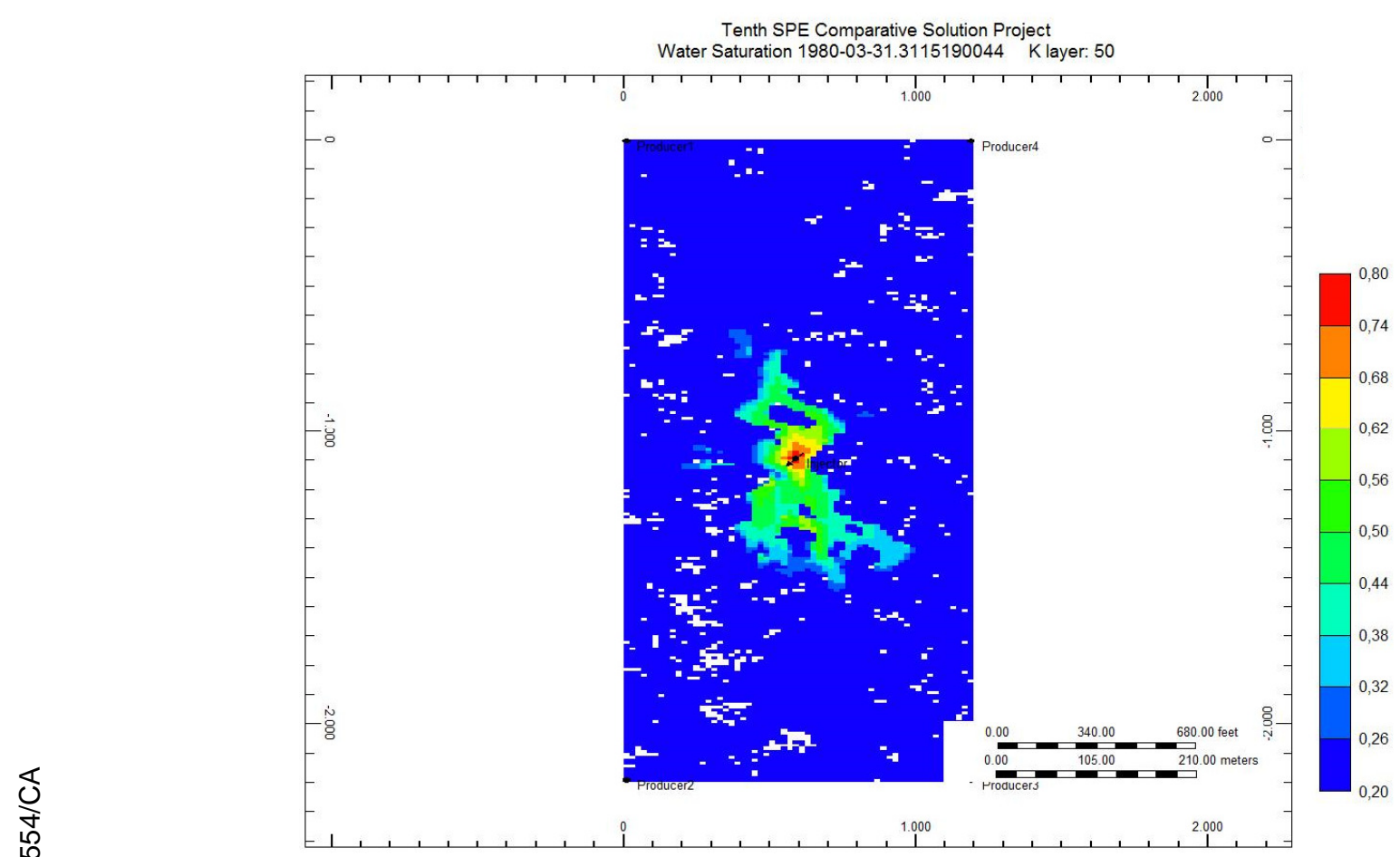

Figura 79: Saturação da água no meio heterogêneo do layer 50 (Três meses depois, aproximadamente)

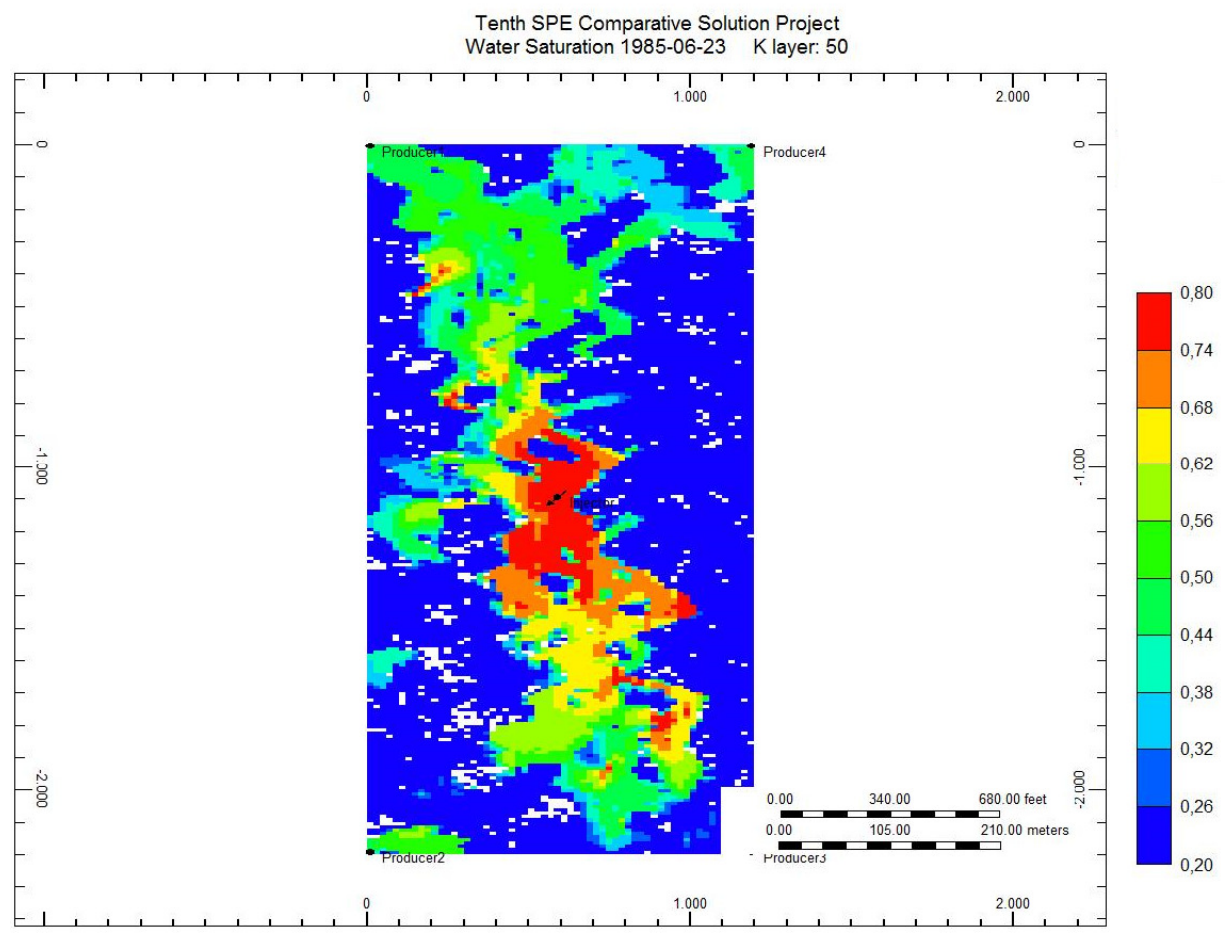

Figura 80: Saturação da água no meio heterogêneo do layer 50 (Cinco anos depois, aproximadamente) 
Note-se também que nas figuras relativas à variação do campo de saturação uma formação Upper Ness, mais precisamente na Figura 80. 